
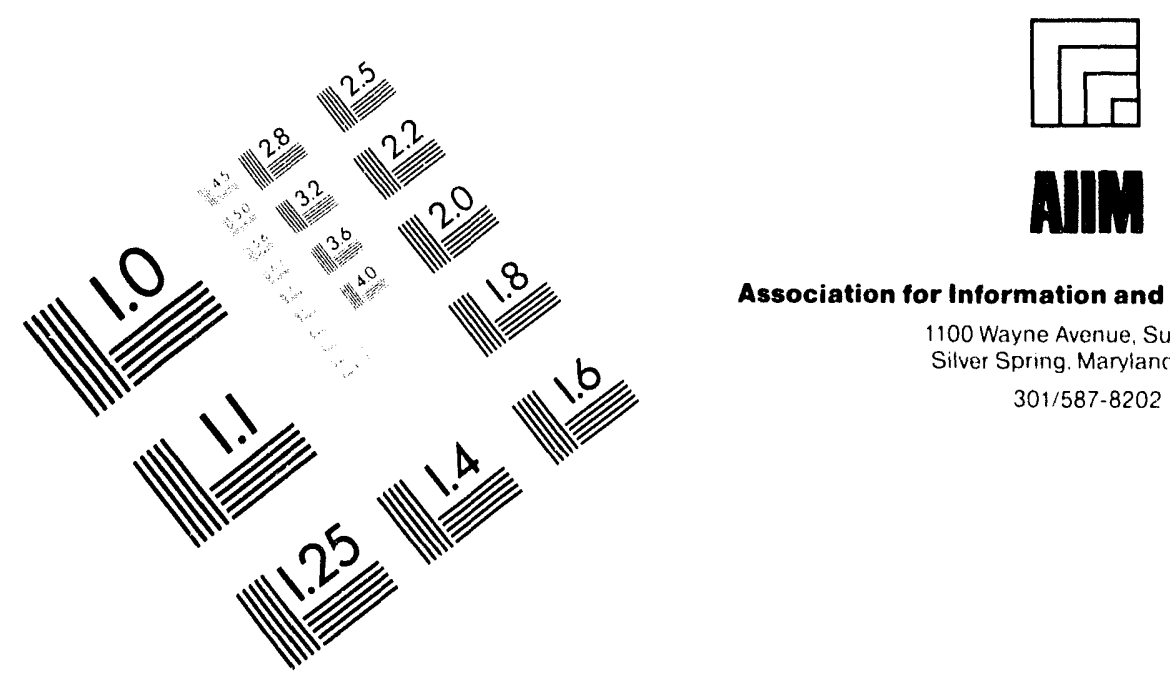

Association for Information and Image Management 1100 Wayne Avenue, Suite 1100 Silver Spring. Maryland 20910 301/587-8202

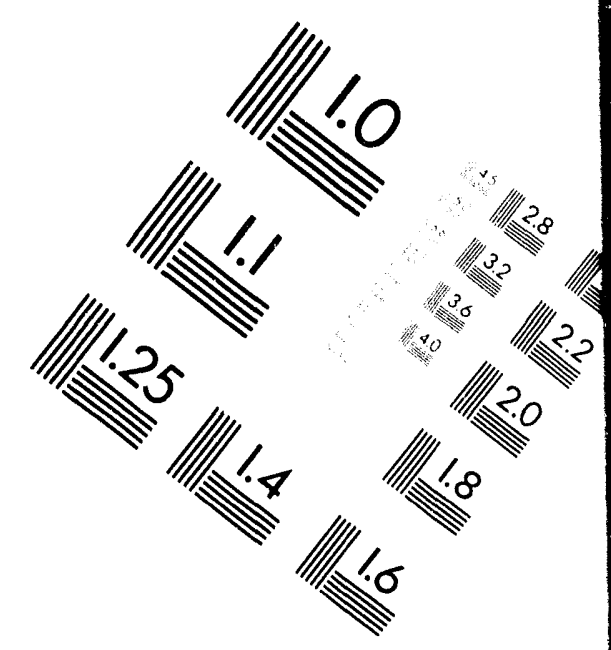

\title{
Centimeter
}

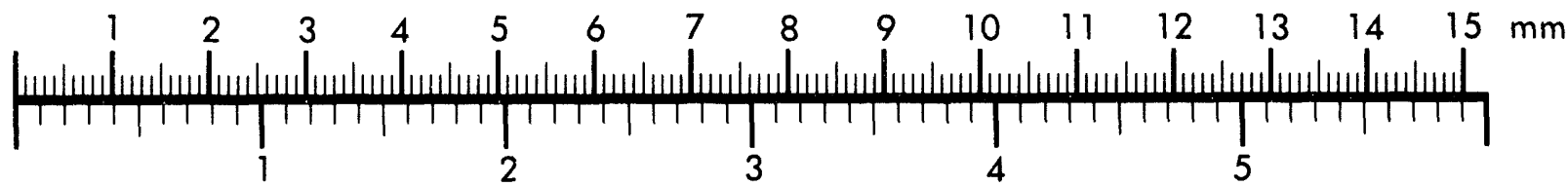
Inches
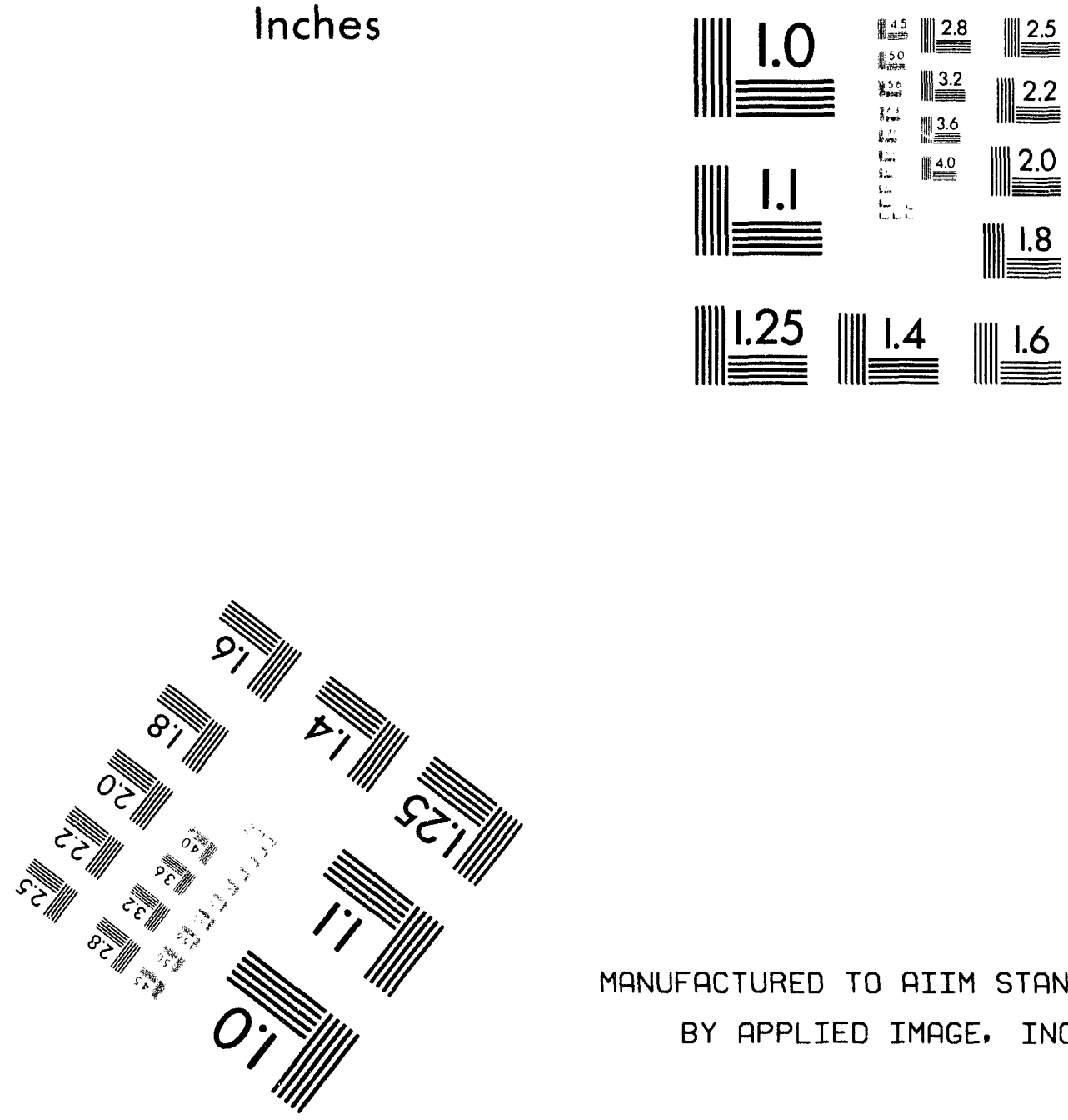

MANUFACTURED TO AIIM STANDARDS

BY APPLIED IMAGE, INC.

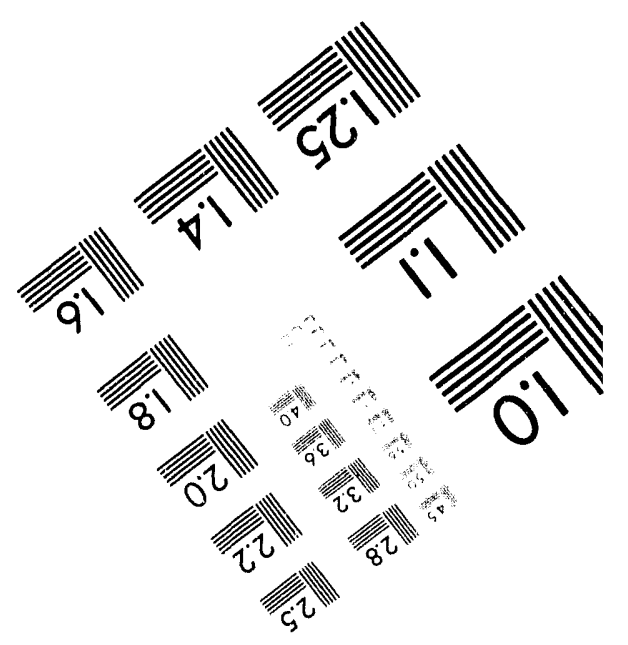



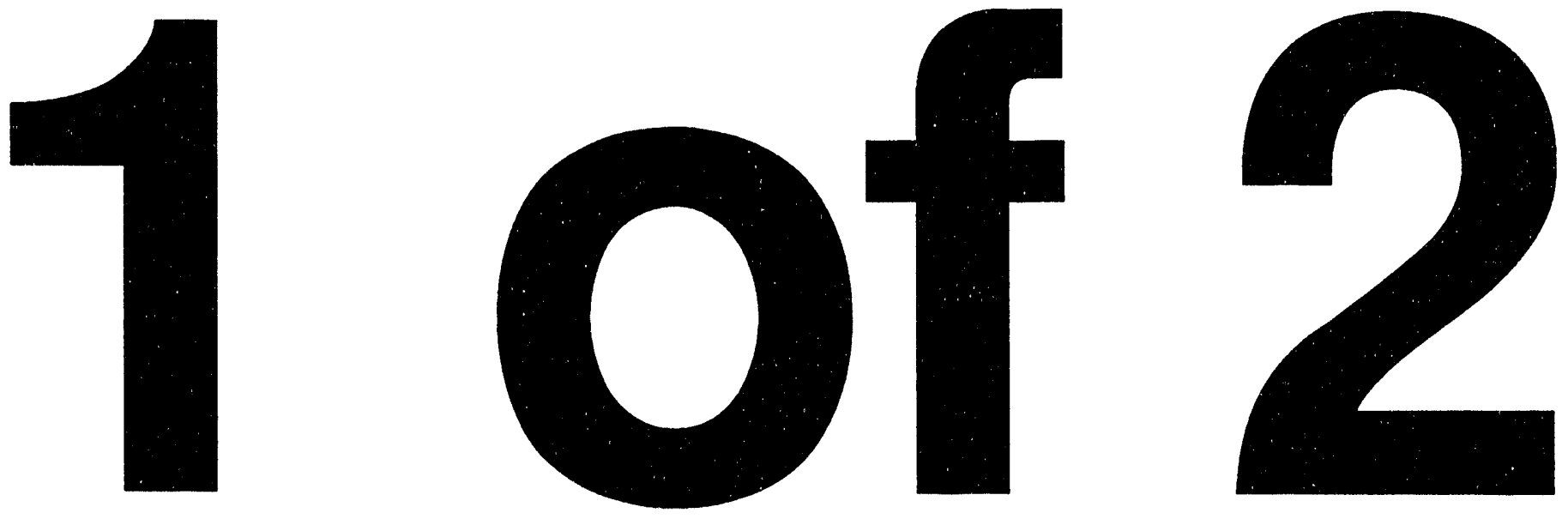


\section{Tank Farm Surveillance and Waste Status Summary Report for December 1993}

Prepared for the US. Dejanment of Energy Oitice of Environmental pestoration and Wasto Management

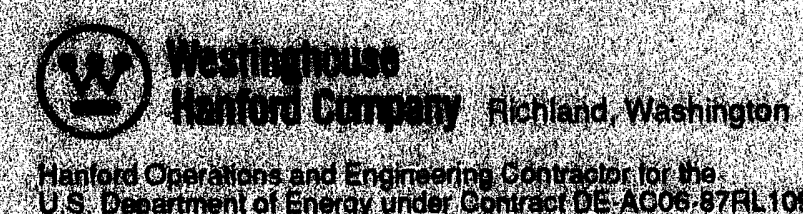

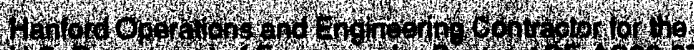

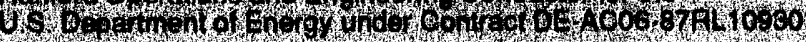

BISTFW UTION OF THWS DOCUNHENT E UNLWTTED Tproved for Public nelease 


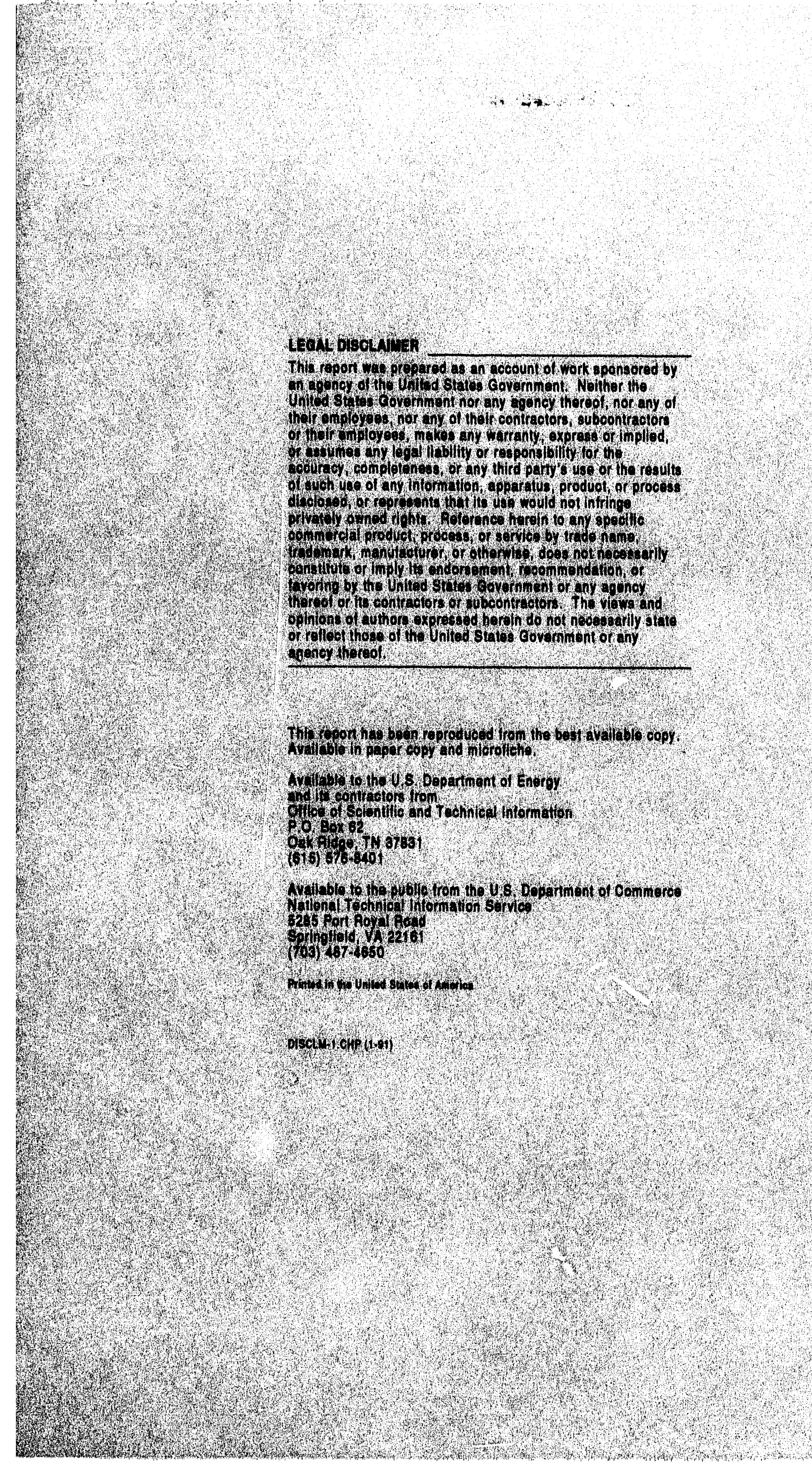
Stalcs Government: Noither th courey, completenes, or any third party s use or the result

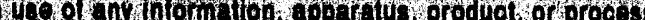
(1)

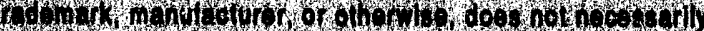
(1) of rellect those of tha United States Govennment of thy agancy thareof:

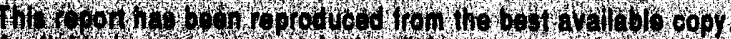

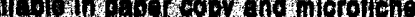

Avits of ro tho US bepartment of Enero

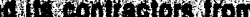

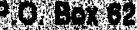

1000

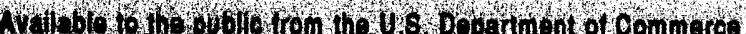
(1)

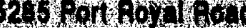

2216

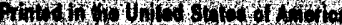

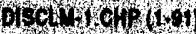




\section{APPROVAL}
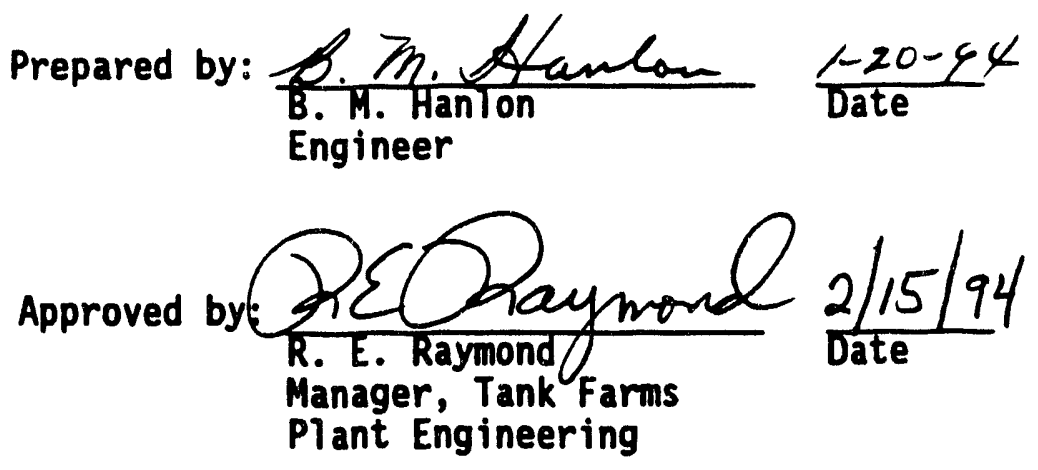

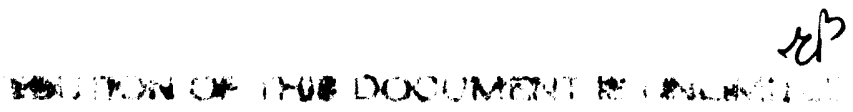




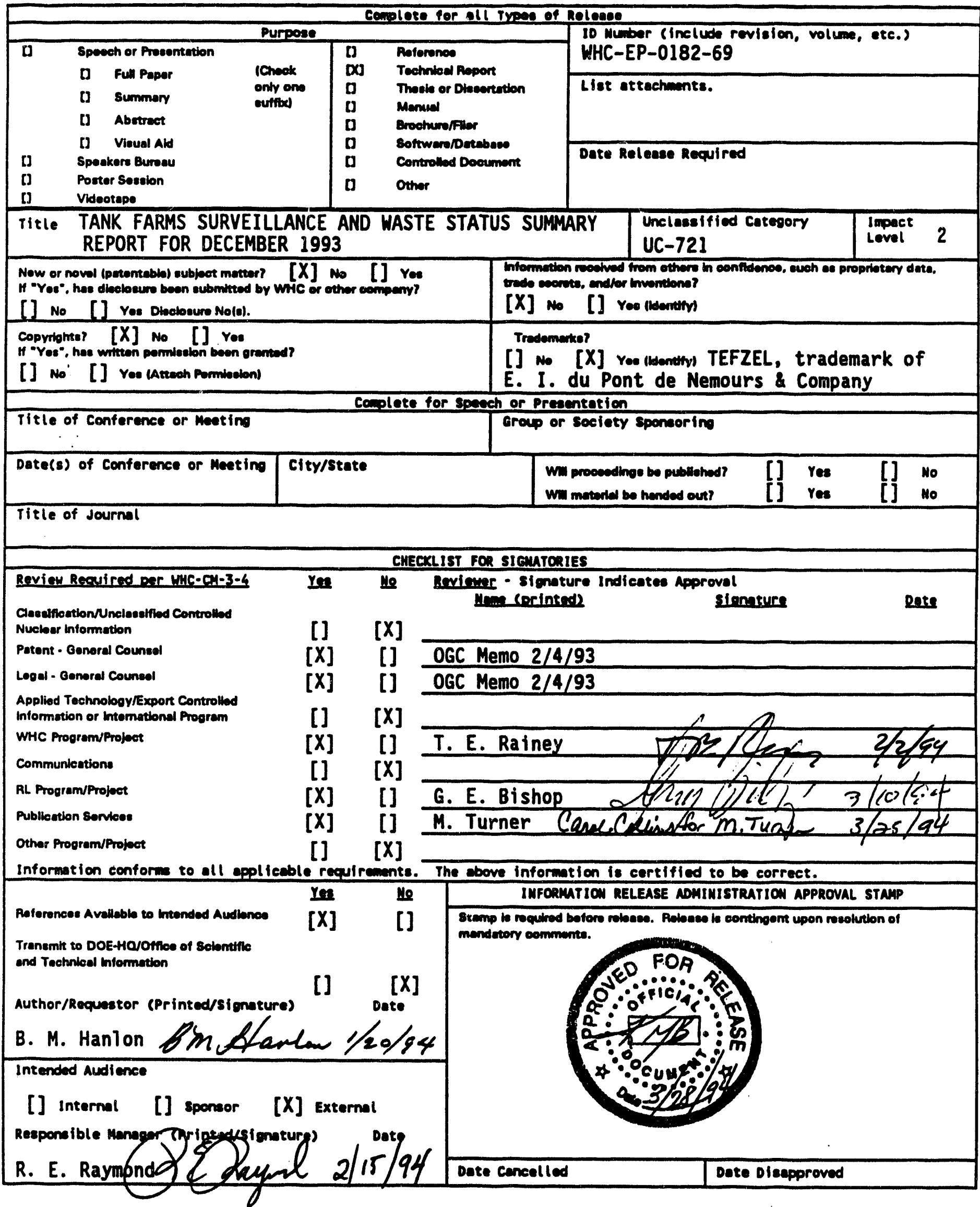


WHC-EP-0182-69

\title{
TANK FARM SURVEILLANCE AND WASTE STATUS
} SUMMARY REPORT FOR DECEMBER 1993

\author{
B. M. Hanlon
}

\begin{abstract}
This report is the official inventory for radioactive waste stored in underground tanks in the 200 Areas at the Hanford Site. Data that depict the status of stored radioactive waste and tank vessel integrity are contained within the report. This report provides data on each of the existing 177 large underground waste storage tanks and 49 smaller catch tanks and special surveillance facilities, and supplemental information regarding tank surveillance anomalies and ongoing investigations. This report is intended to meet the requirement of U.S. Department of Energy-Richland Operations Office Order 5820.2A, Chapter I, Section 3.e. (3) (DOE-RL, 1990, Radioactive Waste Management, U. S. Department of Energy-Richland Operation Office, Richland, Washington) requiring the reporting of waste inventories and space utilization for Hanford Tank Farm Tanks.
\end{abstract}


WHC-EP-0182-69

This page intentionally left blank. 
MHC-EP-0182-69

CONTENTS

SUMMARY . . . ........................ 1

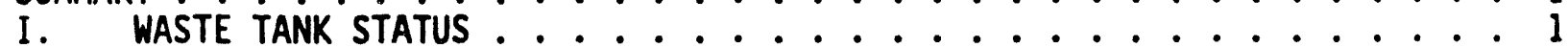

II. WASTE TANK INVESTIGATIONS ....................... 2

III. SURVEILLANCE AND WASTE TANK STATUS HIGHLIGHTS . . . . . . . . . . . 7

Appendixes:

A. WASTE TANK SURVEILLANCE MONITORING TABLES .............. . A-1

Tables:

1 Watch List Tanks . . . . . . . . . . . . . . . . A-3

2 Tanks Containing $>1000$ Gram Mole of Ferrocyanide . . . . . . A-5

3 Tanks With Potential for Hydrogen or Flammable Gas

Accumulation above the Flammability Limit . . . . . . . . . . A-7

4 Single-Shell Tanks Containing Concentrations of Organic Salts - A-8

5 Single-Shell Tanks With High Heat Loads $(>40,000 \mathrm{Btu} / \mathrm{h})$. . . A-10

6 Non-Watch List Low Heat List Tanks $(<40,000 \mathrm{Btu} / \mathrm{h})$. . . . . . A-12

7 Single-Shell Tanks Monitoring Compliance Status . . . . . . . A-15

8 Double-Shell Tanks Monitoring Compliance Status . . . . . . A-20

9 Automatic Food Instrument Corporation (FIC) Gauges

Fiqures:

Out of Service ............... A-22

1 Discrepancy Report Status . . . . . . . . . . . . . A-23

2 Discrepancy Report Cumulative Total . . . . . . . . . . A-24

3 Discrepancy Report Status by Age . . . . . . . . . . . A-25

B. DOUBLE-SHELL TANK WASTE TYPE AND SPACE ALLOCATION . . . . . . . . B-1

Tables:

1 Double-She 11 Tank Waste Type and Space Allocation ...... . B-3

2 Double-Shell Tank Waste Inventory ........... . B-14

Figures:

1 Current Status and Contingency Space for the 242-A

Evaporator Restart .................. B-4

2 Total Available Usable Tank Space . . . . . . . . . . B-5

3 Priority Space Availablity and Usage . . . . . . . . . . . B-6

4 Comparison of Monthly Average Waste Generation to

Management Limit by Facility ............... B-7

5 PUREX Monthly Waste Generations . . . . . . . . . . . . B-8

6 Tank Farm Monthly Waste Generations .. . . . . . . . . . B-9

7 B Plant Monthly Waste Generations . . . . . . . . . . . . B-10

8 T Plant Monthly Waste Generations . . . . . . . . . . . . . . . B-11

9 S Plant Monthly Waste Generations . . . . . . . . . . . . . . B-12

10300 Area Monthly Waste Generations . . . . . . . . . . . B-13

C. TANK AND EQUIPMENT CODE AND STATUS DEFINITIONS ...........

1 Tank and Equipment Code/Status Definitions . . . . . . . . C-3 
WHC EP-0182-69

This page intentionally left blank 
D. TANK FARM CONFIGURATION, STATUS AND FACILITY CHARTS . . . . . . . . . D-1

Fiqures:

1 High-Level Waste Tank Configuration . . . . . . . . . . D-3

2 Double-Shell Tank Instrumentation Configuration . . . . . . D-4

3 Single-She11 Tank Instrumentation Configuration . . . . . . D-5

4 Double-Shell Tank Status ............. . D-7/8

5200 E Single-Shell Tank Status . . . . . . . . . . . . . D- $9 / 10$

$6200 \mathrm{~W}$ Single-Shell Tank Status . . . . . . . . . . . . D-11/12

7 Hanford Tank Farms Facilities Chart: 200-East Area . . . . . . D-13/14

8 Hanford Tank Farms Facilities Chart: 200-West Area . . . . . . D-15/16

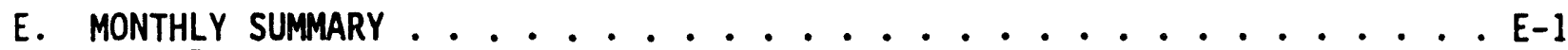
Tables:

1 Monthly Sunmary ...................... E-3

2 Tank Use Summary ................... E-4

3 Inventory Summary by Tank Farm ............. E-5

4 Inventory and Status by Tank - Double-Shell Tanks ....... E-6

5 Inventory and Status by Tank - Single-Shell Tanks ........ E-9

F. PERFORMANCE SUMMARY .................... F-1 Table:

1 Performance Summary ................... . . . . .

G. PUMPING RECORD/LIQUID STATUS AND PUMPABLE LIQUID REMAINING IN TANKS . . G-1 Tables:

1 Pumping Record ... . . . . . . . . . . . . G-3

2 Liquid Status and Pumpable Liquid Remaining in Tanks ....... G-4

H. CATCH TANKS AND SPECIAL SURVEILLANCE FACILITIES . . . . . . . . . H-1 Tables:

1 East and West Area Catch Tanks and Special Surveillance Facilities (Active) ............ H-3

2 East Area Catch Tanks and Special Surveillance Facilities (Inactive) .............. . H-4

3 West Area Catch Tanks and Special Surveillance Facilities (Inactive) .............. H-5

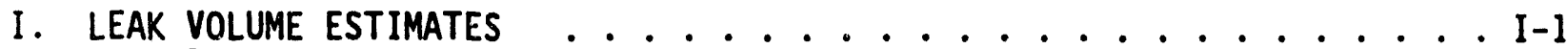
Table:

1 Single-Shell Tank Leak Volume Estimates . . . . . . . . . I-3

J. INTERIM STABILIZATION STATUS ................ J-1 Table:

1 Single-Shell Tanks Interim Stabilization Status ........J-3 


\begin{tabular}{|c|c|c|}
\hline \multicolumn{3}{|c|}{ METRIC CONVERSION CHART } \\
\hline 1 inch & $=$ & 2.54 centimeters \\
\hline 1 foot & $=$ & 30.48 centimeters \\
\hline 1 gallon & $=$ & 3.80 liters \\
\hline 1 ton & $=$ & 0.90 metric tons \\
\hline \multicolumn{3}{|c|}{${ }^{\circ} \mathrm{F}=\left(\frac{9}{5}{ }^{\circ} \mathrm{C}\right)+32$} \\
\hline \multicolumn{3}{|c|}{$\begin{array}{c}1 \mathrm{Btu} / \mathrm{h}=2.930711 \mathrm{E}-01 \text { watts } \\
\text { (International Table) }\end{array}$} \\
\hline
\end{tabular}


MHC-EP-0182-69

\section{TANK FARM SURVEILLANCE AND WASTE STATUS SUMMARY REPORT FOR DECEMBER 1993 \\ SUMMARY}

Note: Changes from the previous month are in bold print.

\section{WASTE TANK STATUS}

\begin{tabular}{|c|c|c|}
\hline Category & Quantity & Date of Last Change \\
\hline In-Service Tanks ${ }^{c}$ & 28 double-shell & $10 / 86$ \\
\hline Out-of-Service Tanks" & 149 single-shell & $07 / 88$ \\
\hline Assumed Leaker Tanks ${ }^{f}$ & 67 single-shell & 7/93 \\
\hline Sound Tanks & $\begin{array}{l}28 \text { double-shell } \\
82 \text { single-shell }\end{array}$ & $\begin{array}{l}1986 \\
7 / 93 \\
\end{array}$ \\
\hline Interim Stabilized Tanks ${ }^{b, d}$ & 106 single-shell & $04 / 93$ \\
\hline Not Interim Stabilized $f$ & 43 single-shell & $04 / 93$ \\
\hline Intrusion Prevention Completed & 98 single-shell & $09 / 91$ \\
\hline $\begin{array}{l}\text { Watch List Tanks } \\
\text { Total }\end{array}$ & $\begin{array}{l}45 \text { single-shell } \\
6 \text { double-shell } \\
51 \text { tanks }\end{array}$ & $\begin{array}{c}12 / 93^{h} \\
6 / 93\end{array}$ \\
\hline
\end{tabular}

- Although oll 149 single-shell tenks were removed from service (1.e., no longer euthorized to receive waste) se of November 21, 1980, the category of wout-of-service" was not established until July 1988.

b of the 106 tanks classified os interin stabilized, 59 are listed as assunad leakers. The total of 106 interim stabilized tenks includes six tenks that do not meet current established supernotent end interstitial liquid stabilization criteria: B-104, B-110, B-119, T-102, T-112, and U-110. (The six tenks did meet the criteris in existerce then they were declared interin stebilized). B-110, B-111, and U-110 are assuned leakers but surveillence date do not show an indication of a continuing leak.

c six double-shell tanks listed as "in service" are currently included on the Hydrogen Watch List and are thus prohibited fram receiving waste in eccordance with wsafoty Meesures for Waste Tanks at Henford Nuclear Reservation," Section 3137 of the Mational Defense Authorization Act for Fiscal Year 1991. Novenber 5, 1990, Piblic Lew 101-510.

d of the 45 single-shell tanks on Watch Lists, 19 hove been Interim Stebilized.

- of the 45 single-shall tenks on Wotch Lists, 28 hove completed Intrusion Prevention (this cetegory replaced Interin Isolation). See Appendix C. Tenk and Equipant Codes and status Definitions, for "Intrusion Prevention" definition.

f Eight of the tenks are both assuned leakers and not Interim Stabilized. See Appendix I, Leak Volue Est imates, for more datails. Tenk $5 x-102$ was declared en essuned leaker in May, and reclaseified as sound in July, 1993. See waste Tank Investigations" section of the July 1993 report for more details.

- See Tables A-1 through A-5 for wore inforwation on Watch List Tenks. Four tenks (5-102, 8X-106, TX-118, U-107) are currently on wore than one Watch List.

h Dates for the Wotch List tanks ore Wofficially addad to the Watch List" dates. See Table A-1, Watch List Tanks, for further information. 


\section{WASTE TANK INVESTIGATIONS}

This section includes all single-shell tanks or catch tanks which are showing surface level or interstitial liquid level (ILL) decreases, or druvelll lateral radiation level increases.

There were no tanks under investigation for surface or ILL decreases or for drywell/lateral radiation increases during December 1993.

A. Assumed Leakers or Assumed Re-leakers: (See Appendix C for definition of "Re-leaker")

This section includes all single- or double-shell tanks or catch tanks for which an off-normal or unusual occurrence report has been issued for assumed leaks or re-leaks. Tanks/catch tanks will remain on this list until either a) completion of Interim Stabilization, or b) the updated occurrence report indicates that the tank/catch tank is not an assumed leaker.

Innk 241-8X-111. This tank was declared an assumed re-leaker on April 30, 1993. Pumping of the tank commenced on October 22, 1993. The surface level measurement was 66.75 inches on December 31, 1993. A total of 54,700 gallons was pumped by the end of December.

Resolution status: Upon completion of pumping, the surface level baseline for the tank will be adjusted. Tanks 241-BX-110 and 111 were removed from the ferrocyanide Watch List on July 9, 1993, on the basis that the tanks contain $<1000 \mathrm{gm}$ moles of ferrocyanide.

Saltwell pumping from BX-111 is halted upon reaching maximum allowable level in 244-BX Double Contained Receiver Tank (DCRT). The contents of the DCRT are then transferred to $A N-101$, and pumping is resumed in $B X-111$. This will be ongoing until pumping is completed for BX-111.

\section{B. Tanks with increases indicating possible intrusions:}

This section includes all single-shell tanks for which the surveillance data show that the surface level or ILL has met or exceeded the increase criteria, or are still being investigated.

Tank 241-B-202. A steady increase in the surface level measurement has been observed since December 1984. The manual tape pencil plummet is contacting liquid. When the quarterly reading was obtained on October 6 , 1992, the level was recorded as 144.75 inches, thus exceeding the 2.00-inch increase criteria from the established baseline of 142.50 inches. The surface level measurement was rechecked on October 9, 1992, (145.50 inches) and October 13 (145.00 inches), verifying the increase and that the criteria had been exceeded. The surface level remained stable at 145.50 inches for December 1993. The monitoring frequency has been increased from quarterly to daily. Occurrence Report RL-WHC-TANKFARM-1993-0024 was issued February 13, 1993. This tank is Sound, Interim Stabilized, and Intrusion Prevention completed.

Resolution status: A photo package was initiated on May 11, 1993, to investigate the possibility of an intrusion. Review of previous photos was inconclusive. New photos are required to determine the actual supernatant 
increase, if any. A temporary baseline was established at 145.25 inches, until the new photos are available.

Tank 241-8X-101. On September 2, 1993, the surface level increased from 10.00 to 12.00 inches. The surface level was 11.75 inches on December 31, 1993. In-tank photographs show the manual tape donut plummet contacting liquid in a shallow pool. This tank is an Assumed Leaker, Interim Stabilized, and Intrusion Prevention completed.

Resolution Status: Comparison of October 1986 photos with November 1988 photos shows evidence of an ongoing intrusion. A work package was initiated October 14, 1993, to obtain in-tank photographs which will be used to inspect the area under the plummet and investigate the possible intrusion. A sitewide in-tank photography program is being formed and photo schedules will be produced upon finalization. A finalized photo schedule is not complete, however, in-tank photos for this tank may be available by late 1995.

Tank 241-BX-103. This tank has shown an erratic increase in surface level measurements since January 6, 1986. On January 18, 1993, the surface level measurement in 103-BX exceeded the 0.50 -inch increase criteria from the reference baseline of 19.50 inches, and was verified on January 20, 1993. Discrepancy Report S\&DA 93-522 was issued January 21, 1993. Occurrence Report RL-WHC-TANKFARH-1993-0036 was issued March 25, 1993. The surface level measurement is currently 20.50 inches. The FIC plumet is contacting liquid as indicated by in-tank photographs taken October 31, 1986. This tank is Sound, Interim Stabilized, and Intrusion Prevention completed.

Resolution status: The current level is greater than that prior to stabilization in November 1983. The tank was previously determined to have experienced an intrusion from 1977 to March 1983 (prior to stabilization). Subsequent isolation was expected to halt the intrusion, however, the intrusion is apparently ongoing. A work package was initiated on May 11 , 1993, to re-seal pits and risers and to obtain in-tank photos. The photos will be used to assess the current stabilization status of the tank. A visual survey of the area was performed to determine possible paths for precipitation to enter the tank. The weather covering on the pits and risers was found in place and undamaged. The existing grade is level and revealed no obvious draining problems. Ongoing design/isolation drawing review revealed that nozzles, floor drains and some transfer lines entering the heel pit have been left open. Photo verification of the intrusion will determine the need to provide additional isolation measures. A finalized photo schedule is not complete, however, in-tank photos for this tank may be available by late 1995.

Tank 241-8Y-105. The ILL is currently at 13.31 feet. The baseline is 13.39 feet. Aithough the surface level and ILL are within the criteria of \pm .11 feet, the data indicates unusual behavior trends that merit continued observation. The monitoring frequency has been increased from quarterly to weekly. The manual tape surface level measurement does not show an increase. This tank is on the ferrocyanide Watch List, an Assumed Leaker, and not yet Interim Stabilized.

Resolution status: The surface level and ILL are displaying behavior similar to $T X-113$ and $T X-115$. The ILL is showing an increase, while the surface level measurement is showing a decrease. This phenomena could be due to either solids dissolution or formation of a depression in the solids beneath the plummet in conjunction with an intrusion. Review of previous photos indicates 
the liquid volume is increasing, although it cannot be verified that the solids level is decreasing. A photo package was initiated to investigate the possibility of intrusion, or solids dissolution. A finalized photo schedule is not complete, however, in-tank photos for this tank may be avallable by late 1995.

Tank 241-5-102. This tank has a history of erratic increases/decreases. Intank photographs taken March 18, 1988, show the FIC plumet contacting an uneven, dry surface. The LOW ILL was within the 0.10 foot tolerance when last scanned on December 29, 1993. Surface level measurement is 204.10 inches, which does not exceed the criteria of 3.00 inches above the basel ine of 202.30 inches.

Resolution status: Photos taken in March 1988, show the surface consists of very uneven solids with scattered liquid pools. Photos taken in 1983 compared with the 1988 photos indicate the waste is shifting, possibly causing a change in surface level under the plumet. Reevaluation of the ILL using the "new method" shows an increasing trend of about 0.4 inches per year. Occurrence Report 77-191 attributed previous intrusions into the tank to drainage from the $S A$ and SB valve pits. Because neither the tank nor the pits are interim isolated, the intrusion is likely ongoing. A photo package was initiated on May 17, 1993, to confirm the ongoing intrusion. This tank is on the hydrogen and organic saits Watch List. A safety evaluation has been issued establishing required controls in ordor to perform photo missions on hydrogen Watch List tanks. Either intrinsically safe camera equipment must be used, or a continuous flamable gas monitoring system must be installed.

Tank 241-S-103. The FIC was out of service from March 8 to May 14, 1993. The surface level increased from 104.15 inches on March 8 to 104.80 inches after FIC repair on May 14, 1993. On August 20, 1993, a temporary baseline of 104.80 inches was established, with a criteria of +2.00 inches. This tank has previously been on report for an increase but showed an erratic decreasing trend the latter part of November 1993. The readings ranged from 104.80 to 103.80 inches. The surface level readings were erratic during December, ranging from 104.10 to 104.40 inches. The level on December 31, 1993, was 104.40 inches. A request has been made for a reference elevation check. The LOW, 1ast scanned December 30, 1993, is within the .08 foot tolerance. This tank is Sound, and not yet Interim Stabilized.

Resolution status: After recalibration of the FIC on June 7, 1993, the surface level (SL) remained stable at approximately 104.65 inches. Historical SL shows a general increase since 1981. The SL has continued to increase since the recent jump following FIC caibration. Normally, recalibration returns the SL to previous levels. Reevaluation of surface level data indicates a steady decrease from 104.90 on November 18, to 104.40 inches on December 31, 1993, meaning the FIC may require another calibration. Evaluation of ILL data back to 1986 using the "new method" analysis reveals an increasing trend of 0.161 inch/year which is further substantiated by a previous intrusion investigation performed in 1984. A work package will be initiated by February 15, 1994, to calibrate the FIC. The in-tank photo package will confirm the ongoing intrusion identified in 1984. A finalized photo schedule is not complete, however, in-tank photos for this tank may be available by late 1995.

Tank 241-TX-111. Although the surface level and ILL measurements do not exceed the criteria, the data indicates unusual behavior trends that merit 
continued observation. This tank is Sound, Interim Stabilized, and Intrusion Prevention completed.

Resolution Status: The ILL trend was re-analyzed for this tank using the new "count rate" method. A steady, significant increase of 1.32 inch/year is evident. Surface level data is showing a decrease. Photos show a dry surface. The surface level decrease is expected to be caused by crumbling of the waste beneath the plummet. Overall, the waste level behavior of the tank is similar to that of $T X-113$ and 115. A photo package was initiated on October 13, 1993, to investigate the possibility of an intrusion. A finalized photo schedule is not complete, however, in-tank photos for this tank may be avallable by late 1995.

Iank 241-TX-113. Although the surface level and ILL measurements do not exceed the criteria, the data indicates unusual behavior trends that merit continued observation. This tank is an Assumed Leaker, Interim Stabilized, and Intrusion Prevention completed.

Resolution status: The technical evaluation of the alert condition in this tank was completed April 14, 1993. The results were inconclusive, with recommendation to accelerate the October 1997 waste characterization of the tank. This characterization is expected to confirm that solids are dissolving, causing an increase in ILL. Acceleration of waste characterization is not possible. Watch List tanks have first priority for core sampling. A photo package has been written. LOW scans and SL readings have been increased from a quarterly to a monthly frequency. A finalized photo schedule is not complete, however, in-tank photos for this tank may be available by late 1995.

Iank 241-TX-115. Although the surface level and ILL measurements do not exceed the criteria, the data indicates unusual behavior trends that merit continued observation. This tank is an Assumed Leaker, Interim Stabilized, and Intrusion Prevention completed.

Resolution status: The technical evaluation of the alert condition in this tank was completed April 14, 1993. The results were inconclusive, with recommendation to accelerate the October 1997 waste characterization. Waste characterization is expected to confirm that solids are dissolving, causing an increase in ILL. Acceleration of waste characterization is not possible for non-Watch List tanks. The 1981 photos show evidence of rain intrusion through a central pump pit riser. However, it cannot be concluded from the 1988 photos that the intrusion is ongoing. This tank will be included in the waste characterization with TX-113. LOW scans are taken weekly, and surface level readings have been increased from a quarterly to a monthly frequency. A finalized photo schetule is not complete, however, in-tank photos for this tank are may be avallable by late 1995.

Tank_241-TY-102. Discrepancy Report S\&DA-92-489 was issued November 9, 1992, when the surface level measurement exceeded the 0.50 -inch increase criterla from the established baseline of 31.40 inches. The increase criteria of 0.50 inch, considered to be an extremely tight tolerance for this tank, was revised to 1.00 inch, which is more in line with other tanks that exhibit similar erratic surface level behavior. The tank has a history of intrusions and icicle-shaped mineral buildup on the FIC plummet. The FIC piummet is contacting a shallow pool of liquid. The surface level measurement is 
currently at 31.70 inches. This tank is Sound, Interim Stabilized, and Intrusion Prevention completed.

Resolution status: This tank is experiencing an ongoing intrusion as is evident by comparison of November 1984 photos with July 1987 photos and an increasing trend in surface level data. A visual survey was conducted to determine possible paths for precipitation to enter the tank. The grade around the tank is level and weather covering is on the pits and intact. The photo package, initiated May 17, 1993, will be required to assess the current stabilization status of the tank, and to assist in determining the possible paths of intrusion. Ongoing design/isolation drawing review revealed that nozzles, floor drains and some transfer lines entering the pump pit have been left open. Photo verification of the intrusion will determine the need to provide additional isolation measures. A finalized photo schedule is not complete, however, in-tank photos for this tank may be avallable by late 1995.

Tank 241-TY-106. Surface level readings were stable during 1990 through 1992, ranging between 13.25 to 13.50 inches. The surface level readings fluctuated between 13.50 to 15.00 inches during 1993. In-tank photographs taken August 22, 1989, show the manual tape pencil plummet contacting a dry cracked surface with discarded tapes near the plumnet. The current surface level reading on Deccuber 31, 1993, was 14.25 inches. This tank is an Assumed Leaker, and Interim Stabilized, and Intrusion Prevention completed.

Resolution status: Two readings reached 15.00 inches on October 2 and October 4, 1993, which constitute a sudden increase from 14.50 inches on August 1, 1993. On October 6, 1993, the readings returned to 14.25 inches. Similar behavior occurred on October 8, 1987, when the surface level jumped to the same level then returned to allowable limits. Photos taken August 22, 1989, show a number of discarded tapes beneath the plummet. Occasional anomalous readings would be expected because of the plummet touching the discarded tapes rather than the dry waste. The monitoring frequency has been increased from quarterly to monthly in order to determine a consistent trend.

S-304 Catch Tank. The S-304 Catch Tank was put into service November 4, 1991. The surface level measurement was recorded as 1.80 inches. The level showed a steady increase until September 30, 1993, when the level was recorded as 56.80 inches. On October 1, 1993, the level decreased from 56.80 to 54.30 inches and continued to decrease to 52.89 inches by December 31, 1993. No liquid has been detected in the sump.

Resolution status. A tank leak is considered highly unlikely at this time since S-304 is located in a concrete vault containing a sump, and the sump has been verified as totally dry. It is doubtful a leak of this size (160 gallons) could bypass the sump completely.

An evaporation study will be done. Until the evaporation possibilities are fully analyzed, the baseline has been left at the peak recorded value (immediately after the July intrusion), and further level changes will be monitored by the use of "temporary" baselines. If evaporation can be confirmed, the tank will be rebaselined accordingly. The FIC has been recalibrated and found accurate, 1.e., no adjustments needed.

241-E/L-151 Vent Station catch Tank. The zip cord surface level reading exceeds the maximum operating limit of 36.00 inches. The manual tape has been out of service since July 7, 1992. A temporary zip cord was installed 
December 16, 1992. The level was 45.50 inches which exceeded the active tank limit of $50 \%$ volume of 40 inches ( 400 gallons). Discrepancy Report S\&DA-92511 was issued December 24, 1992. Transfers are not permitted until the tank is pumped and the level is within limits. A new callbrated zip cord was installed December 16, 1993, and the surface level reading went from 71.00 to 59.00 inches. Discrepancy Report 93-655 was issued December 17, 1993. The zip cord was replaced on December 23, 1993, with a new manual tape that read 60.50 inches. The surface level reading on December 31,1993 , was 61.00 inches.

Resolution status: The catch tank was sampled and a new manual tape was installed on December 23, 1993. Two resolutions are underway:

1) Transfer catch tank waste using existing cross-site lines. Required procedure is in approval stages, USQs and required PCA to be completed by January 15, 1994. Transferring with this method could be ready by January 15, 1994.

2) Transfer catch tank waste by tanker truck. Procedure, USQs, and jumper fabrication needed to begin pumping. Should be ready by January 25, 1994.

UX-302-A Catch Tank. The surface level measurement exceeds the maximum operating limit of 50.00 inches. Discrepancy report S\&DA-92-465 was issued May 12, 1992. The current surface level reading is 66.00 inches, which exceeds the $50 \%$ volume of 54 inches (8840 gallons). The FIC plummet is contacting 1 iquid.

Resolution status: Work packages for the transfer of waste from UX-302-A and the repair of necessary instrumentation are being prepared, and the transfer of waste is being scheduled as a prestart item for the cross-site transfer. A work package has been prepared to sample/pump this catch tank. Transfer will begin after completing Vent Station transfer. Procedures are being prepared.

\section{SURVEILLANCE AND WASTE TANK STATUS HIGHLIGHTS}

\section{Tank 241-U-107 Added to Flammable Gas (Hydrogen) Watch List}

Tank 241-U-107 was considered a safety issue because of the potential for reaction between organic materials and oxidants, such as nitrite and/or nitrate, and is currentiy on the organic salts Natch List. As part of an overall evaluation of tank data, the Flamable Gas Tank Stabilization organization requested that an evaluation be conducted for U-107 because the surface level data indicated that the tank was experiencing slurry growth. The tank is experiencing a slow rise in surface level over a period of time. This behavior is consistent with that of the other tanks in its complex, $U-103, U-105, U-108$, and $U-109$, all of which are on the F1ammable Gas Watch List. The results of the evaluation were reported and it was recommended that U-107 be added to the Flammable Gas Watch List.

An Unreviewed Safety Question (USQ) screening was completed and presented to the Plant Review Committee on December 7, 1993, As a result of this meeting, Facility Manager placed U-107 on the Flaumable Gas Watch List. For further details, see Occurrence Report RL-WHC-TANKFARH-1993-0115 below. 
Tank U-107 is Sound, not yet Interim Stabilized, and Partial Interim Isolated. This tank was constructed in 1943-44, with a 530,000-gallon capacity. It contains 406,000 gallons of high level radioactive waste.

\section{Tank Saltwell Jet Pumping}

Tank 241-BX-110 - Saltwell jet pumping began December 4, 1993, to pump contents of this tank into 244-8X Double Container Receiver Tank (DCRT). As with tank BX-111, saltwell pumping will be halted upon reaching the maximum allowable level in the DCRT. The contents of the DCRT are then transferred to tank $N-101$, and pumping is resumed in BX-110. A total of 4,000 gallons was pumped from BX-110 by the end of December 1993. See Table E-5, Inventory and Status by Tank, for further information.

Tank 241-8X-111 - Saltwell jet pumping began on October 22, 1993, to pump tank BX-111 into 244-BX Double Container Receiver Tank. A total of 23,700 gallons was pumped this month, and 54,700 gallons were pumped by the end of December 1993. See Table E-5, Inventory and Status by Tank, for further information.

\section{Tank Waste Remediation System Safety Initiatives}

The U. S. Secretary of Energy has directed that six safety initiatives be implemented in the Tank Waste Remediation System Program to accelerate the mitigation/resolution of the higher priority waste tank safety issues at the Hanford Site. Forty two milestones were established for accomplishing the inftiatives. In FY 1993 four milestones were completed on or ahead of schedule. One milestone was completed behind schedule. In Decomber 1993, one milestone (2r Define tank conditions required for safe storage of ferrocyanide waste by December 1993") was completed, bringing the total for FY 1994 to three mflestones completed on schedule. Two milestone completions have been missed.

\section{Tank Farms Stand Down (Administrative Hold)}

On August 12, 1993, non-essential work activities in the tank farms were put on administrative hold by WHC senior management until operators, supervisors and managers are retrained to perform their duties in a safe and accountable manner. Minimal essential activities required for safety, monitoring and compliance, including the daily "pump-bumping" of the SY-101 mixer pump, will continue. The administrative hold has impacted several programmatic goals for Tank Waste Remediation System (TWRS). A letter from WHC senior management has been submitted to DOE-RL addressing these programmatic delays. Further corrective actions were addressed in a Tank Waste Remediation System Tank Farm Resumption of Hork Plan, dated September 13, 1993. Contained within the Work Plan is the Integrated Schedule for reinitiating individual work activities on an ongoing basis.

Testing of the SY-101 mixer pump continues to be successfully accomplished. Radioactive waste is being saltwell pumped from BX-111, with completion of the pumping scheduled for the end of January 1994. Many other tank farm work activities have now been fully resumed. In addition, reorganization is taking place to reduce management layers, and a new action plan for total upgrade is expected by February 1994. 
5. Tank 241-SY-101 Mixer Pump

Phase B testing is complete. See OFF NORMAL Occurrence Report RL-WHCTANKFARH-1993-0105 below for more information.

\section{Criticality Safety Issues}

On April 30, 1992, an Unreviewed Safety Question (USQ) concerning criticality safety issues in the Tank Farms was declared to be a reportable event. Unusual Occurrence report RL-WHC-TANKFARM-1992-0037 was issued. A prohibition was placed on all waste transfers into and between the tank farm facilities which is negatively affecting various Hanford programs. On September 1, 1992, the approved Justification For Continued Operations (JCO) was received. This establishes the limitations for all tanks receiving transfers and also excludes any interim stabilization of single-shell tanks until further evaluations are completed and approved by DOE-HQ. On December 15, 1992, the approved JCO was issued as WHC-SD-WM-JCO-001, "Justification for Continued Operations of Hanford High Level Waste Tanks Resulting From the Criticality USQ, 492-CRITSAS."

\section{Tank Farm Sampling Schedule}

The approved, baseline schedule for all tank sampling, including rotary and push-mode core sampling, vapor, grab and auger sampling for FY 1994, is included in this report. This estabilishes the offictal baseline, and any changes to this schedule must be done via change control. See Table 1 following this sumary section - the sampling schedule will appear in this issue oniy, and will be included again when the schedule is revised.

\section{Revised Heat Generation for Single-Shell Tank 241-C-106}

An analysis based on a rovised thermal history for Tank 241-C-106 (from 150,000 to $118,300 \mathrm{Btu} / \mathrm{h}$ ) resulted in a new heat load estimate of 110,000 $B t u / h( \pm 20,000 \mathrm{Btu} / \mathrm{h})$ for this tank. The new heat load is based on the thermal transient behavior during the ventilation outage in 1992. The thermal analysis calculated the temperatures for the entire 1 ife of the tank, 1947 to 1992, plus a 10-year extension to the year 2002. See Table A.5 and footnotes.

\section{Occurrence Reports}

RL-WHC-TANKFARM-1993-0092 (OFF NORMAL) - INSTRUMENT CABLE SEVERED DURING SUBCONTRACTOR EXCAYATION RESULTS IN LOSS OF REMOTE ALARM SIGNALS FROM WESI TANK FARM FACILIIIIES (Final Report. 12/07/93)

On October 22, 1993, a subcontractor to Kaiser Engineers Hanford (KEH) was performing excavation work for piping installation when a Computer Automated Surveillance System (CASS) Iine was severed. The cable was severed by a backhoe while a trench was being dug for treated effluent transfer piping installation. This resulted in the 200 West Area tank farms being without remote alarm monitoring capabilities. Included in the cable severing was a 480 volt power line to the East-West vent station which had no impact to the facility. Electrical utility personnel were immediately dispatched to de-energize the power line. The CASS contingency plan was initiated by operations personnel in accordance with the procedure. 
Previous excavation work conducted by KEH was to identify all known utilities in the area where excavation was to be conducted. However, the CASS cable and power line were not identified. Engineering drawings were provided showing CASS lines but were inaccurate as to location.

A work package was initiated to repair the severed CASS cable. Repairs have been delayed pending WHC company procurement of repair material.

Contributing to this incident was the lack of supervision provided to the offsite personnel by Kaiser Engineers Hanford (KEH). There were no KEH personnel at the job site when the cable was severed. Kaiser line management had the responsibility to ensure that the subcontractor personnel were aware of all utilities, including the physical markers that identified the power 1 ines.

The root cause to this incident were the inadequate administrative controls in place to ensure that all known utilities are identified on the civil engineering drawings being used at the job site. KEH personnel are performing another verification of all drawings to ensure the proper location of all underground utilities.

Einal evaluation: Lessons learned for all personnel is that there is a potential for underground utllities to exist within the tank farm complex. Although these utilities were properiy marked, not all are as easily identified.

RL-WHC-TANKFARM-1993-0103 (OFF-NORMAL) - TANK 101-AY OUT OF LIOUID LEVEL LIMIIS HHEN NEY INTERIM OPERATIONAL SAFETY LIMITS PER HHC-SD-0SR-016 IMPLEMENTED ON NOVEMBER 30, 1993. AT 0001 HOURS (10-DaY Report. $12(14 / 93)$

On November 30, 1993, Waste Tank Operations implemented section 3.1 .2 of the Interim Operation Safety Requirements (IOSR) for Double-Shell Tanks (WHC-SD-0SR-0016, Rev. F). This section states the Limited Condition of Operation (LCO) iimit for Primary Tank Liquid Level is $\leq 416$ inches. It was previously noted that the manual tape for this tank had been giving slack tape measurements above this 1 imit and that an occurrence report would be required upon IOSR implementation. The food Instrument Corporation (FIC) level instrument is currently out of service. The slack tape reading on November 30 was 417.75 inches.

The LCO action statement was entered for Tank AW-101 level. All transfers into or out of this Watch List tank had previousiy been suspended. Action was commenced to restore tank level to with in the specified 1 imit of 416 inches (within 184 days as stated in the LCO). The work package to repair the FIC is being prepared for field work on a priority basis. When the FIC is repaired, the expected level reading should be approximately 410 inches. The steady increase in level by manual tape is consistent with crystalline growth on the tip of the plummet. After the FIC repairs are completed, a work package will be developed to remove the crystalitine bulldup on the manual tape using a series of flushes.

The root cause of this occurrence is Maste Tank Operations' (WTO) lack of a system to expeditlousiy resolve corrective malntenance issues associated with Watch List tanks. WTo is currently providing 
conservative safety analysis of corrective maintenance issues associated with Watch List tanks. By using process knowledge and alternative

(though less dependabie) methods of measuring 1 lquids, wro management is confident tank AH-101 liquid levels have not exceeded operational

Iimits.

RL-WHC-TANKFARM-1993-0105 (OFF-NORMAL) - DISCOVERY OF NEW GAS (METHANE

GAS) IN WASTE TANK 241-SY-101 FOUND WHEN REVIEWING DATA FROM EVENT I AT

LEVELS OF 1000 PPM (10-Day Report. 12/17/93)

On December 2, 1993, MHC Operations was notified by Los Alamos National Laboratories (LANL) that upon further review of the data from Tank SY101 window "I" which occurred on June 26, 1993, wethane gas $\left(\mathrm{CH}_{6}\right)$ was found in the amount of 1000 parts per mililion (ppm) in the tank vapor space. LANL also advised WHC operations that wethane has 3.2 times the heating value of hydrogen $\left(H_{2}\right)$. This in turn adds fuel to the burn analysis in the current. safoty assessment.

All aixer puap activities were halted, with the exception of the pump bump operations, until further resolution was obtained. The Technical Review Group wet with LANL and concurred with the presentation from LANL to continue with mixar puap testing. DOE-RL concurred with WHC to lower the maximu level to 406 inches and continue with pump testing. The control to lower the maximu level to 406 inches was implement on December 2, 1993. The current level of the tank is 400 inches and an auto shut-off is installed that would immediately shut off the pump if the tank level rises two inches during pump bump operations.

The lowering of the maximum level allowable for mixer pump operations from 408 to 406 inches will provide an adequate safety margin bounded within the current safety assessment. The fmpact of the occurrence on the environment, safety and health of workers and the public, is negligible.

RL-WHC-TANKFARM-1993-0107 (UNUSUAL OCCURRENCE) - OPERATORS ENTER 241-SY IANK FARM DURING MIXER PUMP OPERATION RESULTING IN A VIOLATION OF THE SAFETY ANALYSIS REPORI (SAR). (Not if ication Report. 12/06/93)

On December 4, 1993, during Phase B, Test 18 of the SY-101 mixer pump, the Operations Test Coordinator (OTC) observed operators in a restricted area (exclusion area). The orc tmmediately aborted the test and made appropriate notifications.

Investigation rovealed that all safoty requirements were met until shift turnover. The test lasted longer than predicted and the shift manager was notified. He attempted to reach the operators but could not. The operators removed the exclusion area signs and entered the tank farm without inforwing the shift manager that they were planning to do so.

Root cause: The operators should not have assumed they had authority to enter the farm should have informed the shift manager that they were doing so prior to entering the Sy farm. Had the operators called the shift manager and informed him they were removing the exclusion area signs, the shift manager would have been abie to prevent them from doing so, and prevented the event from occurring. 
Contributing cause: Comunications were less than adequate. A formal pre-job brief was not held by the Test Director, Operations Test Coordinator and Shift Manager. Separate turnovers and pro-job briefs were held, and, as a result, information regarding the length of the test was not accurately related to the shift manager.

RL-WHC-TANKFARM-1993-0110 (OFF-NORKAL) - POTENTIAL REACTIVE COMPONENI DISCOVERED IN SINGLE-SHELL WASTE TANK 241-T-11) (10-DaY Report. $12 / 17 / 931$

On December 1, 1993, MHC Tank Farms Plant Review Committee (PRC) reviewed a potentiai unreviewed safety question (USQ) relating to Tank T-111. Core samples taken in 1991 were analyzed in 1992. An uncharacterized reactive component (exotherm) was identified in the sample area. Background information indicated there are data in composite samples taken in 1974 that also show exotherms, however, the data have been questionable.

WHC Operations is treating T-111 like an organic Watch List tank, but will not declare it as a Watch List tank pending further Investigation. Dafly surface level measurcents and weekly temperature data wili be taken. Plant Engineering will review the data reports from other tanks to determine if a sibllar condition exists. Additional analyses on existing samples has been conducted by the Analytical Laboratorles and Pacific Northwest Laboratories (PNL). Partial preliminary data were received Deccuber 17, 1993. Plant Engineering will re-verify all data to determine if there were exotherm releases from core samples.

There has been no significant impact to the facllity or operations.

RL-WHC-TANKFARM-1993-0111 (OFF-NORMAL) - LIOUID LEVEL IN THE EAST/WEST VENT STATION ANNULUS SPACE EXCEEDED 80\% CAPACITY. RESULTING IN AN OSD NONCONFORMANCE TO OSD-T-151-00015 (10-Day Report. 12/22/93)

On December 10, 1993, during a review of operations logs by the Shift Operations Manager, it was discovered that the East/Mest Vent Station catch tank liquid ievel was reading approximately 70 inches (700 gallons), $80 \%$ of the 800-gallon capacity of the tank, resulting in and Operations Specifications Document (OSD) nonconformance to OSD-T-15100015 .

The annulus was checked and verified to have no liquid level. Daily meetings have been scheduled to resolve the proper actions necessary to pump the tank. A work package was initlated to install a manual reel tape in the tank's enclosed housing by December 23, 1993, to determine the exact level in the tank. The manual tape reading will be compared with the previous liquid level measurcments to determine the rate of level increase. A liquid sample of the tank's contents will be taken and analyzed.

RL-WHC-TANKFARM-1993-0115 (OFF-NORMAL) - 200 NEST AREA HASTE TANK 241-U107 ADDED TO THE FLAMABLE GAS MATCH LIST. EXHIBITS SAME BEHAVIOR AS OTHER TANKS ON MATCH LISI (Notification Report. 12/17/93)

In April 1990, the safoty issue of flamable gases began to receive attention with the formal filing of an occurrence report (WHC-UOR-90-TF- 
02). In response to this UOR, interim operating requirements were issued and a list of tanks that may have the potential to generate flammable gases was created. Formal notification of the process used by WHC to select the tanks was provided by letter. It was recognized that when the "Watch List" was set up, more tanks wight be added to the Watch List, as additional data became available, or as additional analyses were performed.

Tank 241-U-107 was considered a safety issue because of the potential for reaction between organic material and oxidants, such as nitrite and/or nitrate, and is currently on the Organics Watch List. As part of an overall evaiuation of tank data, the Flammable Gas Tank Stabilization organization requested that an evaluation be conducted for $U-107$ because the surface level data indicated that the tank was experiencing slurry growth. The behavior of U-107 appeared to be identical to tanks U-103, U-105, U-108, and U-109, all of which were on the Flanmable Gas (Hydrogen) Watch List. The results of the evaluation were reported and it was recommended that U-107 be added to the Flammable Gas Watch List.

An Unreviewed Safety Question (USQ) screening was completed and presented to the Plant Review Comittee (PRC) on December 7, 1993. As a result of this meeting, the Facility Manager placed U-107 on the Flamable Gas Watch List.

The PRC did not feel that there was sufficient information available to permit any action with respect to the USQ screening; additional information will be requested from Haste Tank Safety Programs (WTSP). 
TABLE 1. FY 1994 TANK FARM SAMPLING SCHEDULE (shieet 1 of 4)

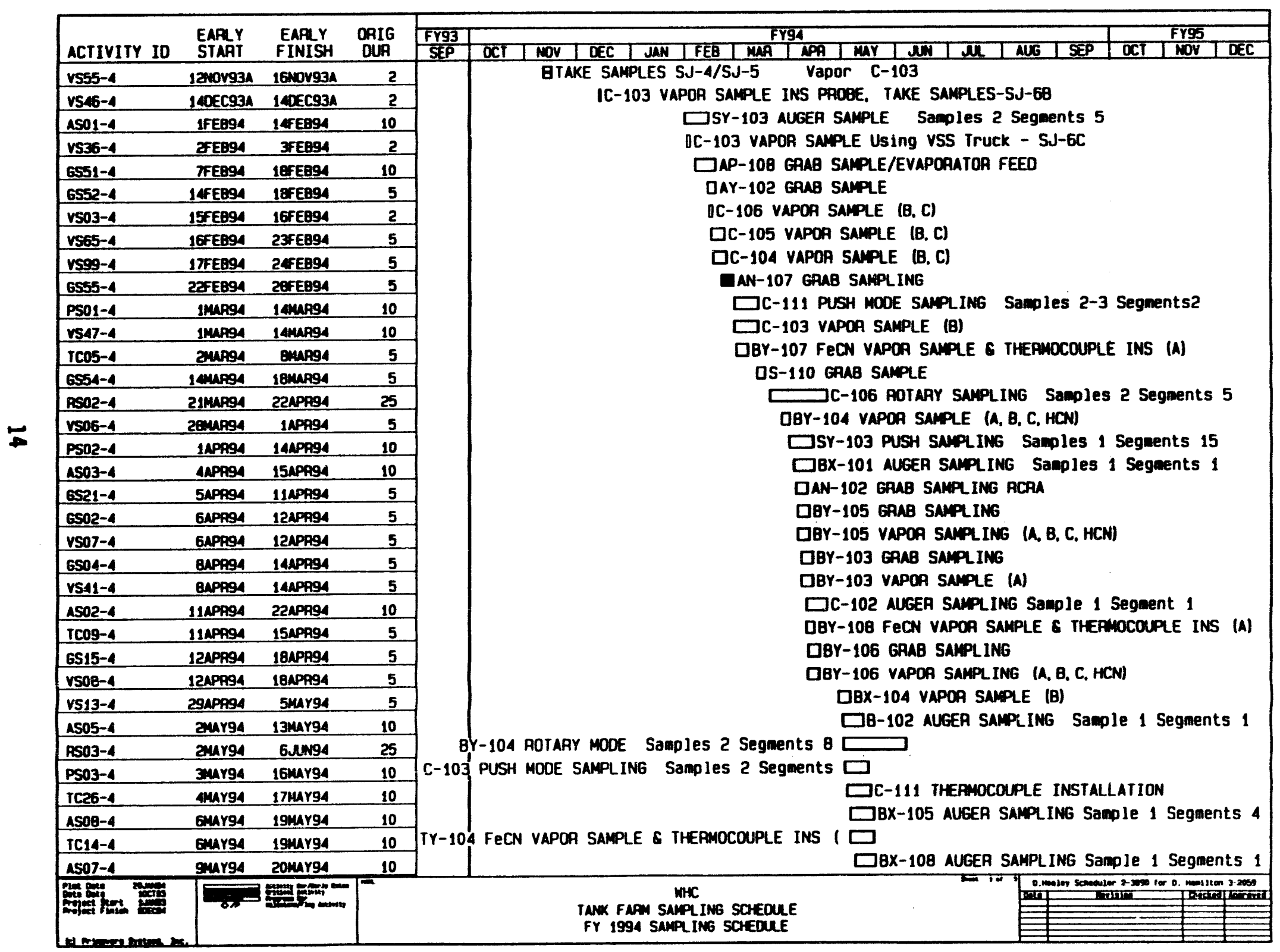

Note: The Key to the letters in parentheses () follows this table 
TABLE 1. FY 1994 TANK FARM SAMPLING SCHEDULE (sheet 2 of 4 )

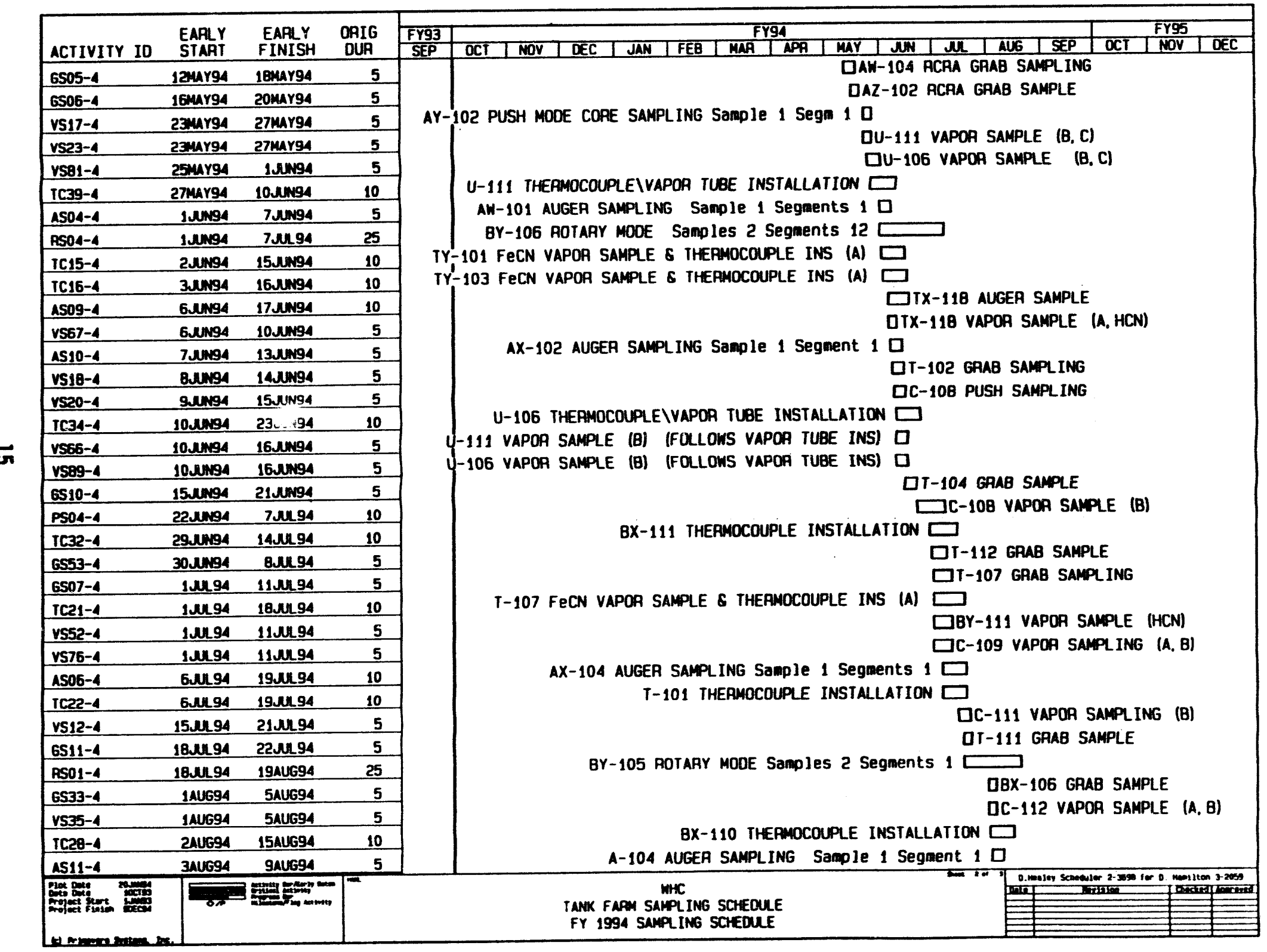

Note: The Key to the letters in parentheses () follows this table 
TABLE 1. FY 1994 TANK FARM SAMPLING SCHEDULE. (sheet 3 of 4)

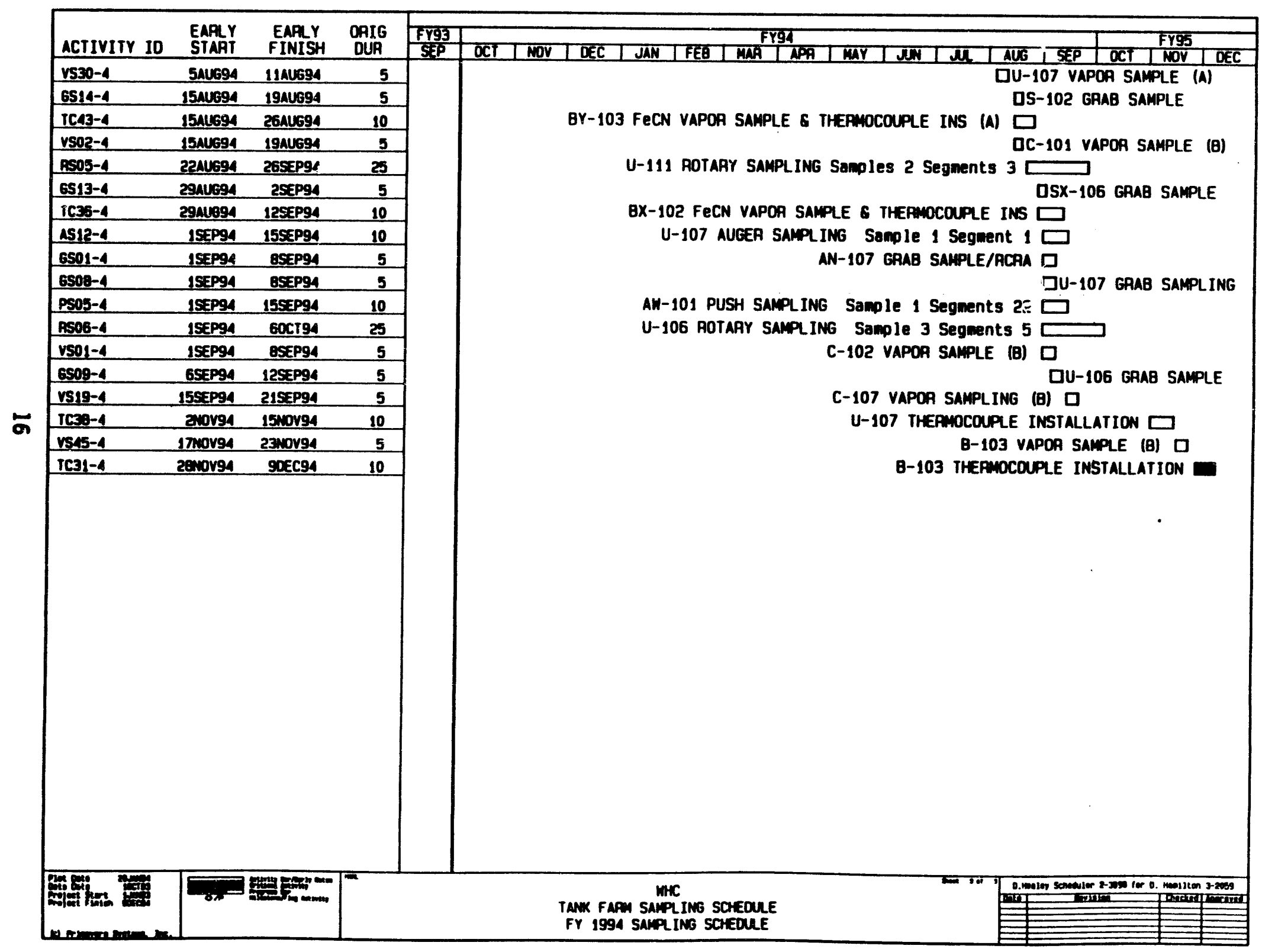

Note: The Key to the letters in parentheses () follows this table 
TABLE 1. TANK FARM SAMPLING SCHEDULE (sheet 3 of 4 )

KEY

A - Ferrocyanide Watch List Flamability and Health Hazards Vapor Sampling (1 day duration)

This type of sampling is required prior to intrusive work in any of the ferrocyanide Watch List tanks. The requirements are explicitly provided by a safety assessment. It involves a check of the flammability of the headspace using a CGM, and a check of the organic vapor concentration using a OVM, at 3 different elevations within the tank. It also currently requires that certain hazardous compounds be sampled for, including hydrazine $\left(\mathrm{H}_{6} \mathrm{~N}_{2}\right)$, ammonia $\left(\mathrm{NH}_{3}\right)$, hydrogen cyanide $(\mathrm{HCN})$, and nitrogen oxides (NOX). These hazardous compounds are measured at each of the 3 levels, using Draiger tubes.

B - Heated-Tube Vapor Sampling by the Tank Vapor Issue Resolution Program (1 day duration)

The Vapor Program has developed the vapor sampling system (VSS) to collect representative vapor samples from the tanks. This system uses a heated sampling probe, heated sample transfer lines, and a heated manifold to eliminate the condensation of vapors. This type of sampling is to be performed to resolve health and safety issues. Each sampling event will be designed to obtain a minimum amount of data on a set of analytes, and to address questions unique to each tank. Currently all ferrocyanide and organic salts Watch List tanks are to be sampled by this method.

C - Exhauster Air Permit Vapor Sampling to support exhauster use during Rotary Mode Core Drilling

(1 day duration)

Use of an exhauster that pulls air from the waste tanks will je part of the rotary mode core drilling operations. To obtain an air permit from the state to operate the exhauster, WHC has agreed to sample and analyze the vapors in the tank prior to operating the exhauster. To date, no tank has been sampled for this reason. The initial tanks to be rotary mode core drilled will be sampled by the Tank Vapor Issue Resolution Program, using the heated probes and manifold of the Vapor Sampling System. Sampling and analysis will target all gases and vapors normally of interest to the Tank Vapor Issue Resolution Program. Methods for future vapor characterization to support exhauster use have not been identified.

D - Special Vapor Sampling to address special issues (duration unknown at this time)

Flammability check of tank 103-C. The flammability of the tank 103-C headspace cannot be measured by the same means as other tanks, since a fog of organic liquid droplets may exist in the tank. The chosen method of determining the headspace flammability will involve sampling the semivolatile organic vapor and aerosol. After samples have been collected there is an anticipated 3 week delay for sample analysis.

HCN - Hydrogen Cyanide Sampling - Impinger method (1 day duration)

NOTE: All activities will require 1 day duration for prep work and 1 day for clean up.

Also, all organic tanks listed above will have the flamability taken at the breather filter which is different from the method performed on FeCN tanks. Since the time to perform the flamability check takes less than 20 ainutes, the duration to perform the flamability for any watchlist tanks has been absorbed into the duration for that activity. 
NAC-EP-0182-69

This page intentionally left blank 
WHC-EP-0182-69

\section{APPENDIX A}

\section{WASTE TANK SURVEILLANCE MONITORING TABLES}


WHC- [P-0182-69

This page intentionally left blank 
TABLE A-1. WATCH LIST TANKS (Sheet 1 of 2)

These tanks have been identified as Watch Lbt Tanks in accondance with Public Law 101-510, Section 3137. - Safety Maeserres for Waste Tanks at Haniond

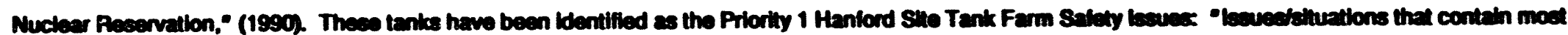

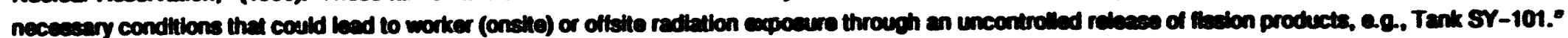

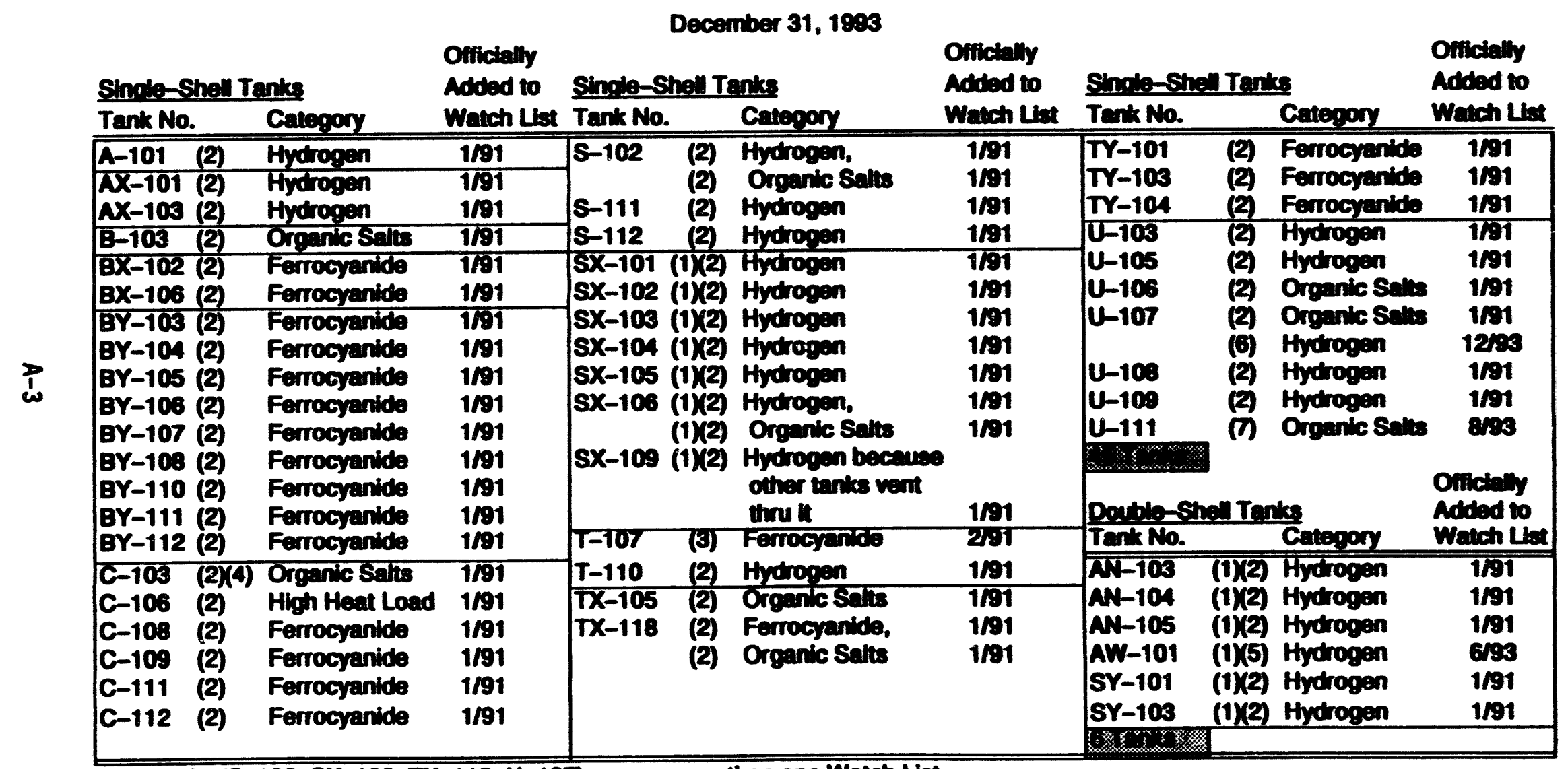

Four tanks (S-102, SX-106, TX-118, U-107) are on more than one Watch List

See footnotes next page 
Footnotes:

(1) These eight single-shell tanks and the six double-shell tanks on the Watch List are actively ventilated.

Although on various dates beginning in March 1990, WHC identfiled tants containing ferrocyanido, organic salts, etc., which were then added to this report as Watch List tanks, the following official notilications were made to DOE-RL:

Letter 8059124, H. D. Harmon, WHC, to R. E. Gerton, DOE-AL, "Salety Measures for Wadte Tanks at Hanford Site, Plichland, Washington," dated January 8, 1991, Identified 23 ferrocyanide tanks, 23 tanks with potential for accumelation of flammeble gas, elght organic tanks, and one high heat load tank, as being Watch Liat tanks. (52 tanke)

The ferrocyanide and hydrogen tanks were dectared Unresolved Safety Quections (USO); the organic tanks and the high heat load tank were within the safety envelope as defined by the safety anabycis reports and were not dealgnated as USOs.

(3) Letter S059124.1 (revision to 9059124 above), dated February 8, 1991, added T-107 to the Ferrocyanide Watch Lat. (53 tanka)

(4) Tank C-103 was dectared a USQ per Unusual Occurrence Report RL-WHC-TANKFARM-1992-0000, lesuced September 1992,

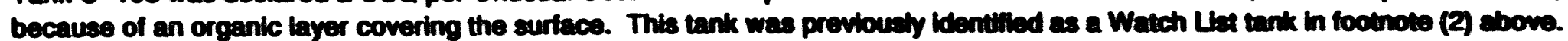

(5) Letter 9354700, J. C. Fution, WHC, to R. E. Genton, DOE-RL. "Addition of Tank 241-AW-101 to Flammable Ges Watch Liat," dated June 3, 1993, added this double-shell tank to the Watch List. (54 tanks)

Letter 9353957, J. C. Fulton, WHC, to R. E. Gerton, DOE-RL, "Single-Sholl Wave Tank 241-U-111," dated May 24, 1993, recommended this tank be inctuded on the Organic Tanks Watch List. Thls tank was added to the Watch List on Auguest $31,1993$.

(7) Tank U-107 was dectared a USQ per Occurrence Report RL-WHC-TANKFARM-1993-0115, beued December 1993. because of an increase in sturry growth. This tank is also on the Organics Watch List.

Notes: BX-110, BX-111, BY-101 and T-101 were removed from the Ferrocyanide Watch Lbet in Juby 1993, per letter 93-CAB-223, John H. Anttonen, DOE-RL, to T. M. Anderson, WHC, "Resohution of Unreviewed Salety Question for Four Ferrocyanide Tanks," dated July 9, 1993. 
TABLE A-2. TANKS CONTAINING >1000 GRAM MOLE OF FERROCYANIDE (Watch List Tanks) (Sheet 1 of 2 )

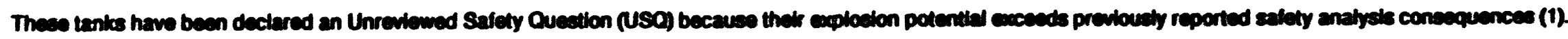

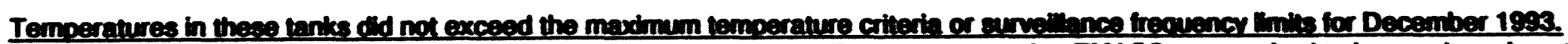

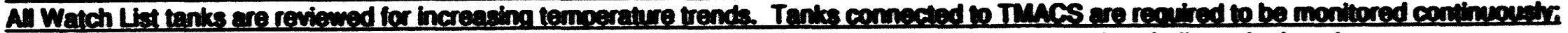

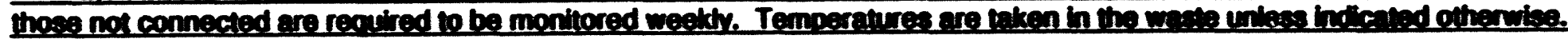

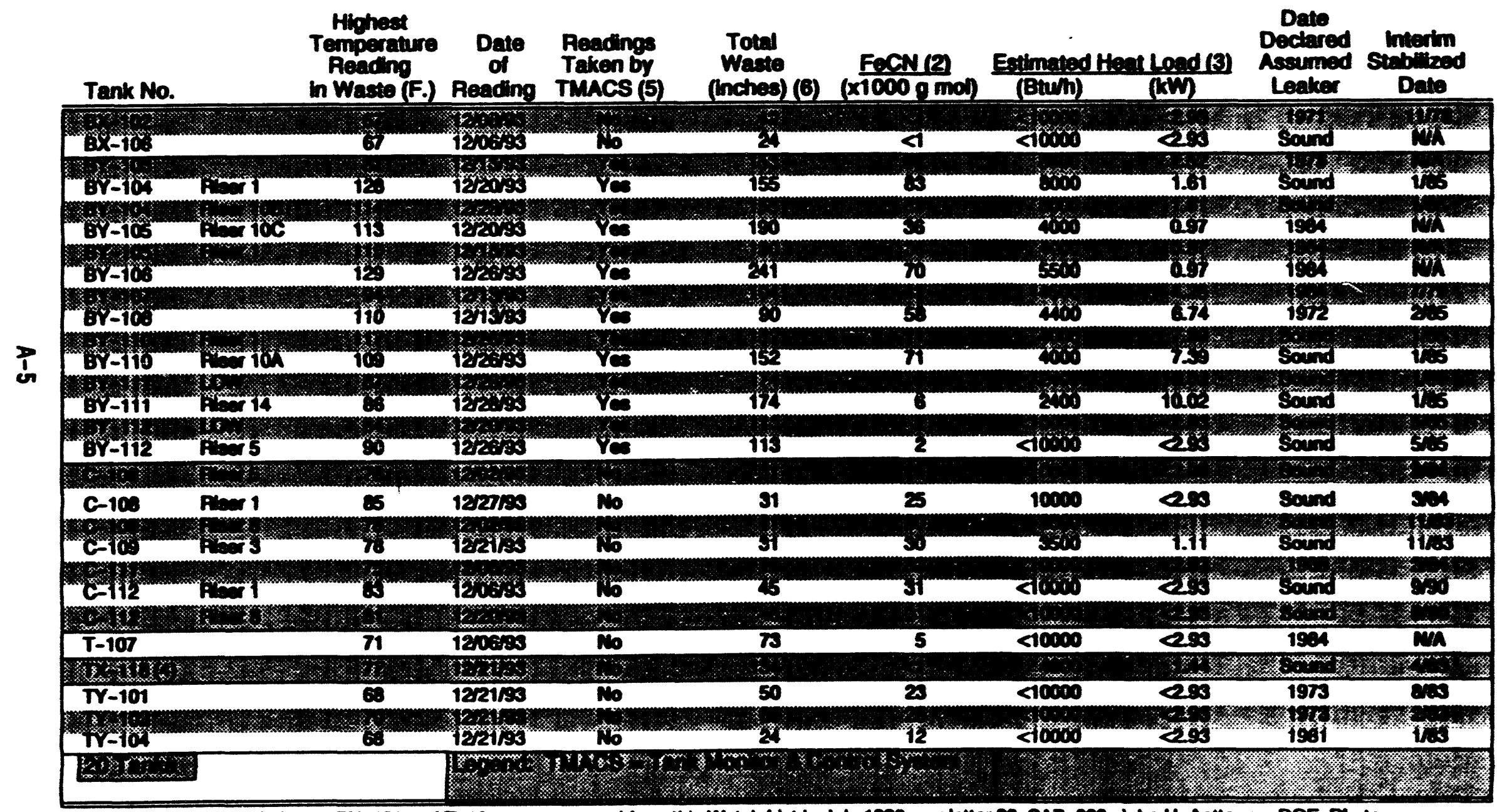

Mater Tents BX-110, BX-111, BY-101 and T-101 were removed from this Watch Llet in July 1993, per letter 93-CAB-223, John H. Anttonen, DOE-Ph, 10 T. M. Anderson, Wic. "Recolition of Unreviowed Salety Ouedion for Four Ferrocyanide Tanks," dated July 9, 1993. FOOTnOTES: Seo neud paco 


\section{TABLE A-2. TANKS CONTANING >1000 GRAM MOLE OF FERROCYANIDE (Watch List Tanks) (Sheet 2 of 2)}

\section{FOOTNOTES:}

(1) This Priority 1 Waste Tank Safety lasue is stated as follows: "Could the concentratisns and distribution of ferrocyanide and nitrate/nitrite in the tanks lead to an explosion if allowed to heat up or if an uncontrollad exothermic reaction could occur?"

(2) The anount of FoCN reported in the tanks is based on WHC-SD-WM-ER-133-AEV O. "An Ascosament of the inwentionies of the FeCN Watch List Tanks," (Table 3-7). October 1891.

(3) The extimated heat generation rates are from the following: WHC-EP-0474-4, "Ouarterty Report on Delense Nuclear Fachleses Salety Board Recommendation s0-7 for the Period Ending March 31, 1992," Table 1-A, dated demuary 1892. Tank 104-BY only eeetunated per WHC-EP-0689. "Ferrocyanide Safety Progrem: Updated Thermal Anabyds Moded for Ferrocyenide Tentes with Applicallon to to Tank 241-BY-104," December 1993. WHC-EP-0038, "Ferrocyanide Salety Program: Heat Load and Thermal Characterletlos Determinalion for Selocted Tanks," November 19e3, anabred stx tanks, BY-105, 108, 103, 110, and 111, and C-109.

(4) This tank abo contains a high concentration (P3\% in TOC) of organic salts.

(5) This column indicates which tanks are being monitored by the Tank Monitor 8 Control Syatem (TMACS); automatic temperature reading: are taken continuousty. Temperatures in tanks connected to TMACS but temporarty not being monitored by TMACS are taken manually. Tanks TX-118, Y-101, TY-103, and TY-104 are being taken manualy in December because of a Tuacs connection matiunction.

(6) Total waste in Kgal taken from Table E-5, Imentory and Stutus by Tentes for SSTa. Konlinches calculations for the temperature tables are as follows: (waste in inches is an approximation only for these temperature twblea)

$$
\frac{\text { Koal wane }-12.5 \mathrm{Koal} \text { wate* }}{2.75 \mathrm{Kgalinch}}+12 \text { inches* }
$$

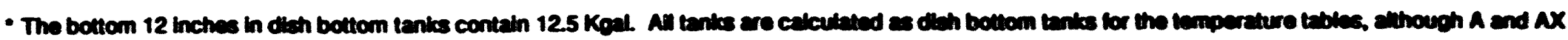
tarms have flat bottoms. inches are from centertine tank bottom. 


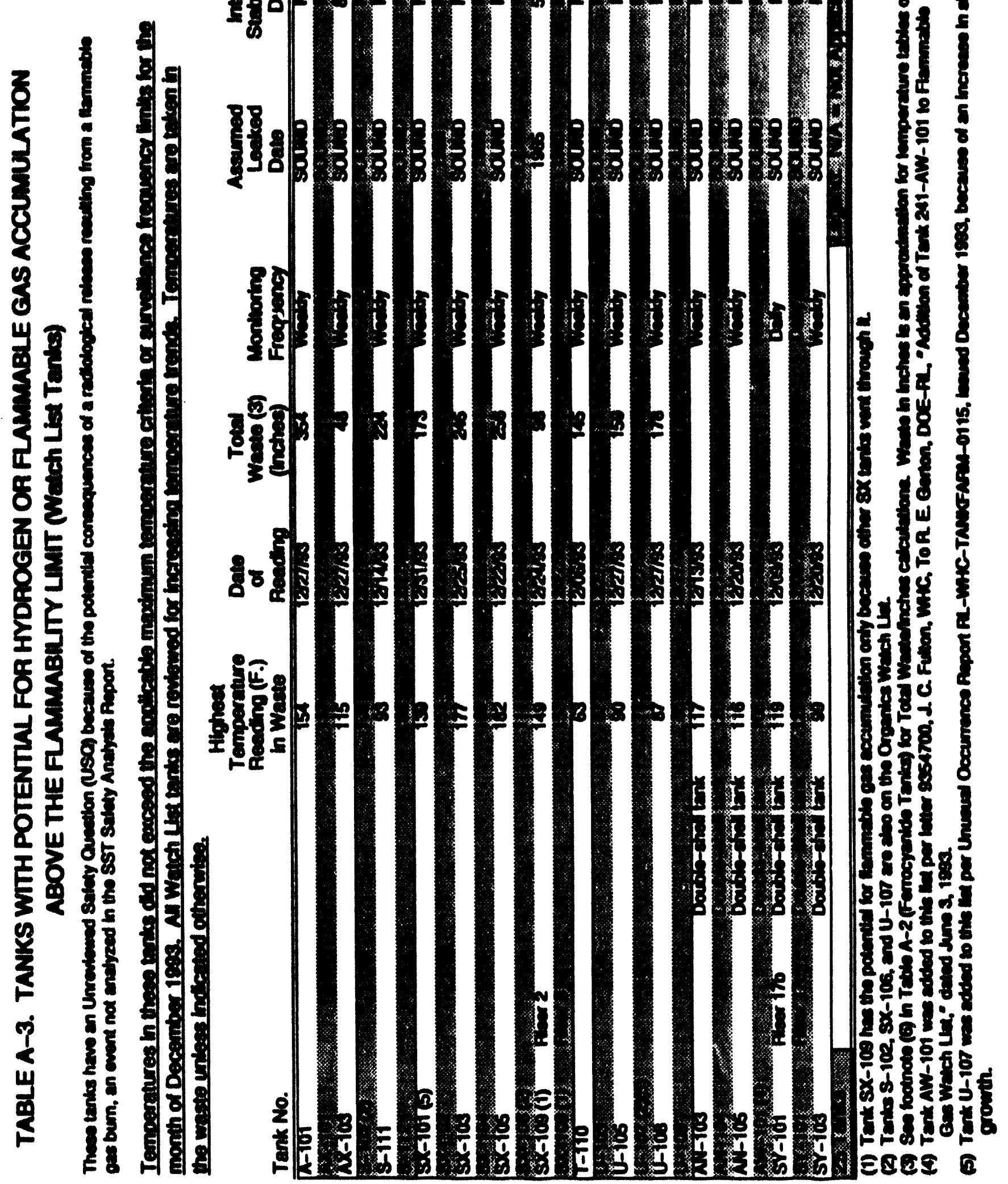


TABLE A-4. SWGLE-SHEL TANKS CONTANUNG CONCENTRATIONS OF ORGANC SALTS $>3$ WEIGHT \% TOTAL ORGANIC CARBON (TOC) Wach List Tenles) (Sheet 1 of 2 )

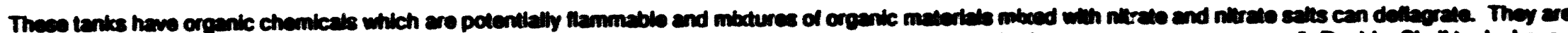

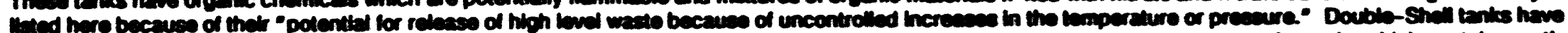

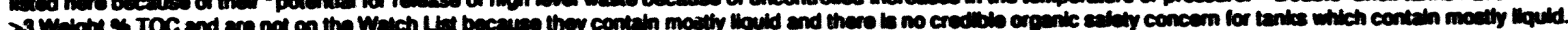

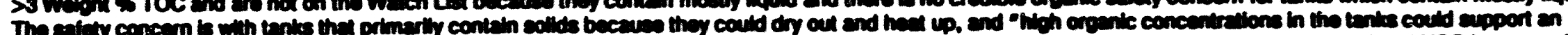

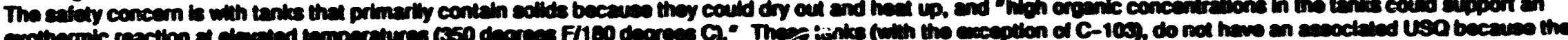

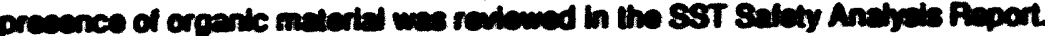

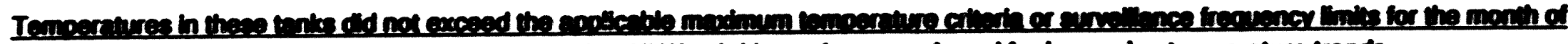

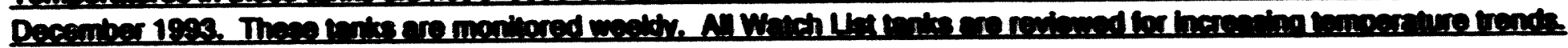

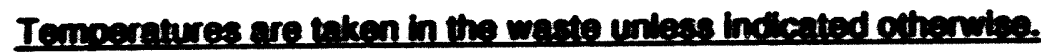

\begin{tabular}{|c|c|c|c|c|c|c|c|c|c|c|c|}
\hline Terik No. & $\begin{array}{l}\text { Temperature } \\
\text { Panding (F.) } \\
\text { in wan }\end{array}$ & $\begin{array}{l}\text { Don } \\
\text { of } \\
\text { Panding }\end{array}$ & $\begin{array}{l}\text { Toted } \\
\text { Wunte } \\
\text { Inemes (4) }\end{array}$ & $\begin{array}{l}\text { Acouned } \\
\text { Lened } \\
\text { Dolo }\end{array}$ & 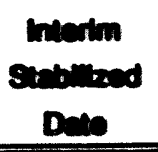 & Souce of wench & 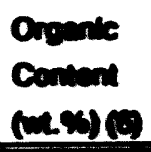 & $\begin{array}{c}\text { and } \\
\text { mano } \\
\text { (nescese }\end{array}$ & $\begin{array}{c}\text { Toc } \\
\text { (1).s) } \\
(n) \\
\end{array}$ & 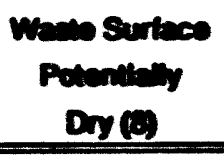 & Son \\
\hline $8-103$ & 6 & 120193 & 2 & 1970 & 205 & 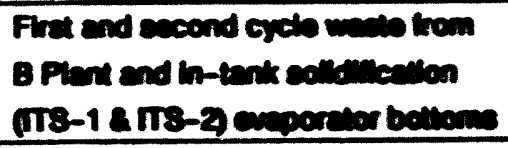 & 11.46 & $\operatorname{cose}$ & $3 \mathbf{3}$ & $x$ & 8975 \\
\hline C-10s $(A)$ & 121 & razdis & 7 & Sovin & $m$ & 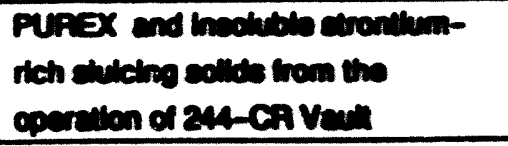 & - & - & - & & 890 \\
\hline $8-102(1)$ & 103 & $12 x_{1103}$ & $2 m$ & sound & $m$ & Feoox & $21.0(10)$ & $41.0(10)$ & a.1 & $\mathbf{x}$ & 200 \\
\hline $8 x-103(1)$ & 114 & 12218s & 200 & soun & $\operatorname{men}$ & 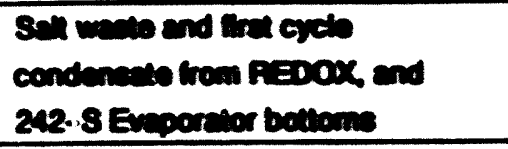 & 1469 & $\cos (\theta)$ & 4.3 & & 879 \\
\hline $\mathrm{Tx}-105$ & 102 & 12003 & 220 & $19 \pi$ & 803 & 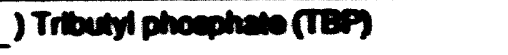 & 1200 & $527 \theta$ & 3.7 & $x$ & $1 \mathrm{mr}$ \\
\hline$T x-118 R$ & $\pi$ & $1821 \times 3$ & 194 & soun & 403 & J procens waste end 242-T & $20.2(10)$ & $50.4(10)$ & 5.9 & $x$ & 891 \\
\hline U-103 & 2 & 1215es & 90 & soino & ma & J Expormer botions & $486(10)$ & $524(10)$ & 136 & & en \\
\hline$U-107(1)$ & $\pi$ & 12220103 & 155 & sound & $m A$ & 1 & $14.7 \mathrm{~B}$ & $75.4 \theta$ & 4.3 & & 1274 \\
\hline$(1-111 \theta)$ & 81 & 121s:s & 127 & soun & $\mathrm{ma}$ & Concentrued 8 Plent Woete & $422(10)$ & ---110 & 14.1 & $x$ & 7193 \\
\hline
\end{tabular}

Sae Fooludes neat pors 
TABLE A-4. SNGLE-SHELL TANKS CONTANWG CONCENTRATIONS OF ORGANIC SALTS $>3$ WEIGHT $\%$ TOTAL ORGANIC CARBON (TOC) (Shaet 2 of 2 )

\section{Footnotes:}

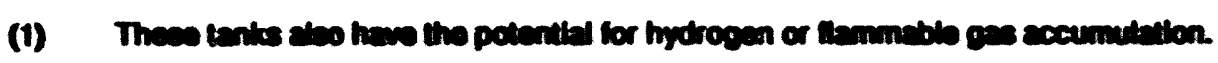

(4) Tank TX-118 eloo contenthe ferrocyande.

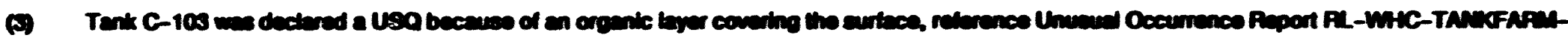
1992-0000, bound Seploniber 1902

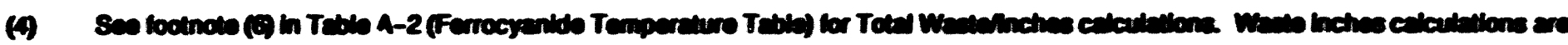
epprodimations ondy lor tempereture tablece.

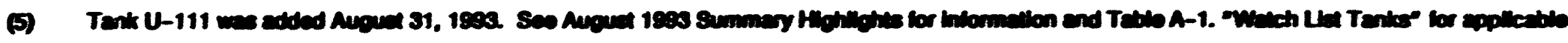
reference

$\mathfrak{l}_{\mathfrak{b}}$

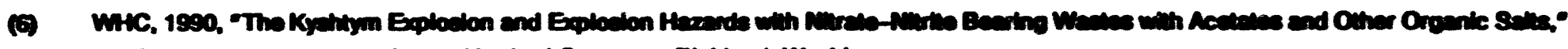

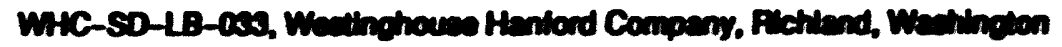

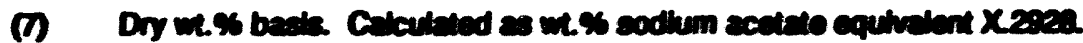

(6) Dus to sbeences of expernatent llquid.

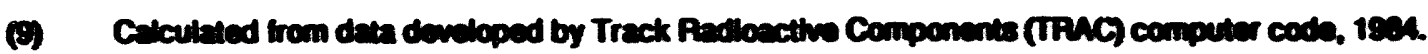

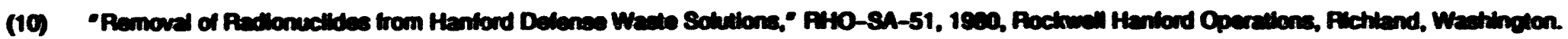

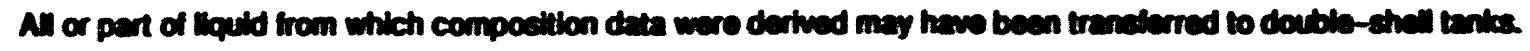

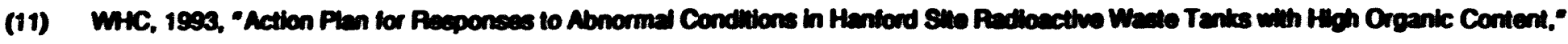
WHC-EP-0461, Rev. 1, Westinghouse Hantord, Fichiend, Weatingtion. 


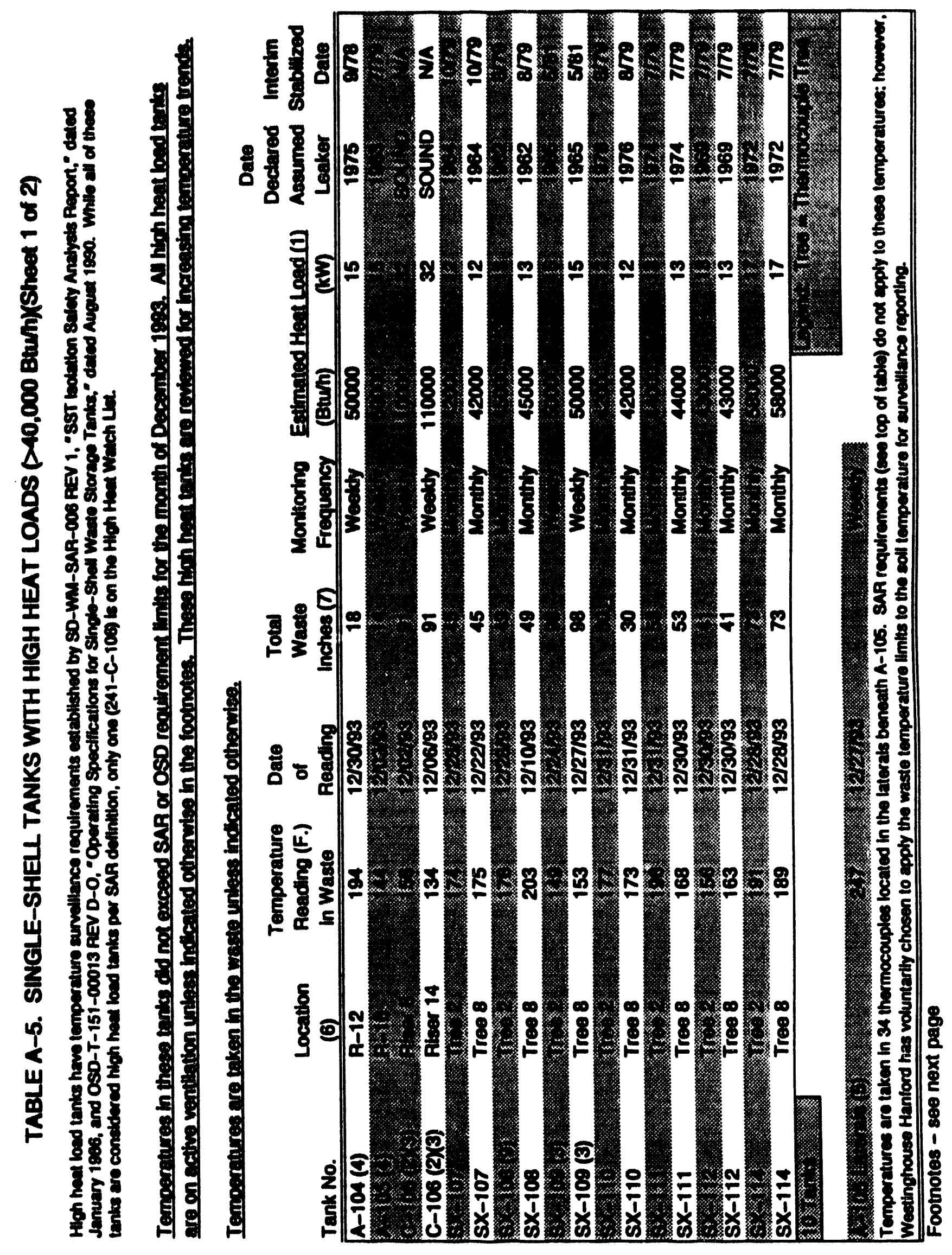


TABLE A-5. SINGLE-SHELL TANKS WITH HIGH HEAT LOADS $(\mathbf{4 0 , 0 0 0}$ Btu/h)(Sheet 2 of 2)

\section{Footnotes:}

(1) High heat loads as of 1988, evalustion completed Aprll 20, 1999 (kW = 3412 Btum). The predominant heat load lor theas tanks is from CS 137 (malf ifto of 30 years) and SA 80 (nalf uff of 28.1 years). Tank C-105 was re-evaluated in WHC-SD-WM-ER-178, "Thermal Anatrals of Tank 241-C-105 in Support of Process Teat," January 1993. Englneering Change Notice 196834 , Juno 24, 1993, changed the status of C-105 from High Heat Loed to Normal, effective July 1, 1993. Tank C-106 was re-waluated ueing a revised thermal history besed on the thermal trandent behavior during the ventilstion outage in 1992. WHC-SD-WM-EA-200

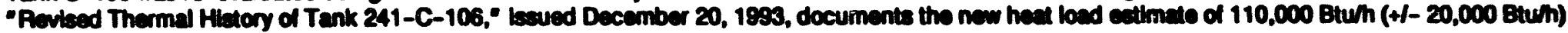
for this tank.

(2) Perlodic water additions are required in C-106 to maintain evaporatwe cooling and thus prevent overheating. This tank is echeduled for partial retrleval starting in 1997, at which time cooling water additions will be dbcontinued. Temperaturces in Riber 8 aro condatent at mid-150 degrees, however, temperatures in rieer 14 very between approximately 120 and 135, aleo condidently.

(3) Watch Liat Tankes: C-106 bs on the Watch Llet because "without water additions (in the event of a leak) the tank could exceed structural temperature firnits

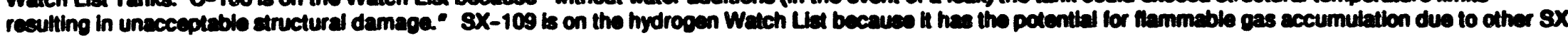
tanks venting through $\mathrm{H}$.

(4) A-104105/106 exhauster has been out of senvice from October 1, 1991, unill Auguat 20, 1992, when Hines brietly reetarted. Problems exiat which must be resolved belore the exhauter boperational. Temperatures in A-104 and A-105 are monilored weeddy.

(5) Maximum lateral temperatures under A-105 increased 20 decrees F. by January 1992, but then dropped a fem degreas and have remained ialing atable at current temperature. These temperatures are monitored weeddy.

(G) Tanka A-104 and A-105

Two temperature probes are installed in rbers in A-104, and atx are installed in rbees in A-105. Theee are individud probes. In A-104, the probes are in contace with the studge; in A-105, they are in contact with the bottom of the tank (A-105 has a buloed bottom).

\section{Tenk C-106}

Tank C-106 has atx functioning thermocouples ( 11 through 6 ) on rber 8, and 12 functioning thermocouples on.tber 14.

Tanks SX-107, 108, 109, 110,111, 112, and 114

Each of these tanks has eloht thermocouple trees, with dofh thermocouples on cach tres, with the ceception of 8X-108, which has four operalonal thermomocouples on each of two trees. Tree 22 and Tree 18 are monthored in each of these $8 \times$ tante.

(n) Calculations for Total Wadte Inches. see footnote (6). Table A-2 (Ferrocyanide Tanks). Wade in inches is en epproudmation only for temperature tables.

(8) There are 19 angle-shell tanks with sctive ventliation (elght are on the Watch Lba as indicated by an asterlak):

\begin{tabular}{|c|c|c|}
\hline $\begin{array}{l}A-104 \\
A-105 \\
A-106 \\
C-104 \\
C-105 \\
C-108\end{array}$ & $\begin{array}{l}\mathbf{S x}-101 \\
\mathbf{S x}-102 \\
\mathbf{S x}-103 \\
\mathbf{S x}-104 \\
\mathbf{S x}-105 \\
\mathbf{S x}-106\end{array}$ & $\begin{array}{l}5 x-107 \\
5 x-108 \\
5 x-109 \\
8 x-110 \\
8 x-111 \\
8 x-112 \\
8 x-114\end{array}$ \\
\hline
\end{tabular}

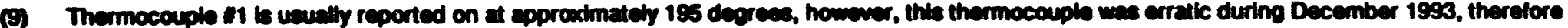
thermocouplo 12 was weed in this report. 
TABLE A-6. NON-WATCH LIST LOW HEAT LOAD TANKS $(<40,000 \mathrm{Btu} / \mathrm{h})$

(Page 1 of 3)

Temperatures are taken semiannually in January and July, unless otherwise indicated, in the following 96 single-shell tanks. Legend follows table.

\begin{tabular}{|c|c|c|c|c|c|c|}
\hline \multirow[b]{3}{*}{1} & \multirow{3}{*}{$\frac{\text { Tank No. }}{\text { A-102 }}$} & \multicolumn{2}{|c|}{$\begin{array}{l}\text { Highest Temperatures } \\
\text { taken in waste }\end{array}$} & \multicolumn{3}{|c|}{ Total Waste (1) } \\
\hline & & $\operatorname{Lan} .93$ & Jul. 93 & \multicolumn{2}{|c|}{ Kal Inches } & \multirow[t]{2}{*}{ Comments } \\
\hline & & 92 & 92 & 41 & 15 & \\
\hline 2 & $A-103$ & 117 & 117 & 370 & 135 & \\
\hline 3 & $A-106$ & 138 & 137 & 125 & 50 & \\
\hline \multirow[t]{2}{*}{4} & $9 x-102$ & O/8 & 76 & 39 & 10 & Only TCus working on 7/93, reading in vapor space \\
\hline & & & & & & Control box contaminated indde \\
\hline 5 & $2 x-104$ & 97 & $\mathbf{8 8}$ & 7 & 3 & \\
\hline 6 & B-101 & 110 & 108 & 113 & 48 & \\
\hline 7 & B-102 & 62 & 63 & 32 & 18 & TCint OS, reading in vepor space \\
\hline 8 & B-104 & 68 & 66 & 371 & 142 & \\
\hline 9 & B-105 & 68 & 66 & 306 & so & \\
\hline 10 & $8-106$ & 61 & 62 & 117 & 67 & TCH1 \& 2 OSS \\
\hline 11 & $8-107$ & 62 & 62 & 165 & 41 & TCH1 thru 3 OS, reading in vepor epses \\
\hline 12 & B-108 & 62 & 62 & 24 & 54 & \\
\hline 13 & B-109 & 62 & 61 & 127 & 97 & \\
\hline 14 & B-110 & OS & 68 & 246 & 94 & TCh1 thru 4 OS. Rasding in vapor space \\
\hline 15 & $\mathrm{~B}-111$ & Os & 88 & 237 & 19 & TCin1 \& 2 OS, work order lewed, historical readings erratic \\
\hline 16 & $\mathrm{~B}-112$ & 62 & 63 & 33 & 7 & \\
\hline 17 & B-201 & 58 & 60 & 29 & 151 & \\
\hline 18 & B-202 & 59 & 61 & 27 & 141 & \\
\hline 19 & B-203 & 62 & 62 & 51 & 263 & \\
\hline 20 & 8-204: & 59 & 62 & 50 & 258 & \\
\hline 21 & $\overline{B x-101}$ & Ors & as & 43 & 27 & All TCs O/S, work order lesued, last reading 74 F. in 11/92 \\
\hline 29. & BX-103 & Ors & Ors & 66 & 31 & All TC. O/S, last reading $\pi 7$ F. In $10 / 92$ \\
\hline \multirow[t]{2}{*}{23} & $B x-104$ & Ors & Ors & 99 & 43 & (2) No TC tree per Riser Configuration document \\
\hline & & & & $\because$ & & Last reading 87 F. In $10 / 80$ \\
\hline 24 & $B x-105$ & 66 & 63 & 51 & 26 & \\
\hline 25 & $8 x-107$ & Ors & O/s & 345 & 133 & All TCB Ors, work order losued, last reading 69 F. in $10 / 82$ \\
\hline 26 & $8 x-108$ & Ors & 63 & 26 & 17 & No relay box $\ln 1 / 93$ \\
\hline 27 & BX-109 & Ors & $\pi$ & 193 & 78 & No relay box in 1/93, no historical readinges avallable \\
\hline 28 & $8 x-110$ & - & 68 & 199 & 80 & Raadings taken weekdy, tank being pumped \\
\hline 29 & $B x-111$ & - & 65 & 230 & 91 & Raedings taken weekty, tank being pumped \\
\hline 30 & $B x-112$ & Os & 62 & 165 & 67 & No relay box in 1/93 \\
\hline 31 & BY-101 & - & 75 & 387 & 148 & Continuous readings taken on TMACS \\
\hline \multirow[t]{2}{*}{32} & BY-102 & as & Ors & 341 & 131 & (2) No TC tree per Rieer Configuration document. \\
\hline & & & & & & Lag reeding 72 F. In 479 \\
\hline & BY-109 & Os & Ors & 423 & 161 & (2) No TC tree per Rileer Configuration document. \\
\hline
\end{tabular}




\section{TABLE A-6. NON-WATCH LIST LOW HEAT LOAD TANKS $(<40,000 \mathrm{Btu} / \mathrm{h})$} (Page 2 of 3)

Temperatures are taken semiannually in January and July, unless otherwise indicated, in the following 96 single-shell tanks. Legend follows table.

\section{Highest Temperatures}

taken in waste Total Waste (1)

Tank No. Jan. 93 Jul. 93 Kgal Inches Comments

\begin{tabular}{|c|c|c|c|c|c|c|}
\hline 34 & C-101 & 91 & 88 & 88 & 39 & \\
\hline 35 & C-102 & OS & Ors & 423 & 161 & Broken coupling, work order issued, last reading 96 F. in 11/92 \\
\hline 36 & C-104 & 88 & 87 & 295 & 115 & TCH1 thru 5 Ors, randing in vapor epace \\
\hline 37 & C-105 & - & 82 & 150 & 63 & Montiored weakly. Formerty on High Heat Load list \\
\hline \multirow[t]{2}{*}{38} & C-107 & - & 125 & 275 & 107 & Monthly reading requirement per procedure, monitored \\
\hline & & & & & & woekly \\
\hline 39 & C-110 & 65 & 65 & 187 & 75 & TCW1 thru 4 OSS, reading! "apor epace \\
\hline 40 & C-201 & 60 & 61 & 2 & 13 & \\
\hline 41 & C-202 & 58 & 61 & 1 & 8 & \\
\hline 42 & C-203 & 58 & 60 & 5 & 29 & \\
\hline 43 & C-204 & O/s & as & 3 & 18 & No hookup to tree, last reading prior to '91. \\
\hline 44 & S-101 & ors & 115 & 427 & 162 & TCA1, 3,5, 6 OS, work order lavued \\
\hline 45 & $\mathrm{~S}-103$ & 88 & 85 & 248 & 88 & \\
\hline 46 & S-104 & 108 & 104 & 294 & 114 & \\
\hline 47 & S-105 & 7 & 73 & 456 & 173 & \\
\hline 48 & S-106 & 81 & 78 & 543 & 205 & \\
\hline 49 & S-107 & 106 & 107 & 368 & 129 & \\
\hline 50 & S-108 & 87 & 85 & 604 & 227 & \\
\hline 51 & S-109 & 83 & Ors & 568 & 214 & All TCs ONS \\
\hline 52 & S-110 & 117 & 116 & 390 & 149 & \\
\hline 53 & $5 x-113$ & 75 & 73 & 26 & 15 & \\
\hline 54 & $\$ x-115$ & OVs & ors & 12 & 10 & (2) No TC Junction box, Iast reading prior $12 / 91$ \\
\hline 55 & $T-101$ & - & 66 & 102 & 45 & Formerly on ferrocyanide Watch List \\
\hline \multirow[t]{2}{*}{56} & $T-102$ & o/s & ovs & 32 & 19 & (2) No TC tree per Riser Configuration document. \\
\hline & & & & & & last reading 68 F. in $2 / 81$ \\
\hline 57 & $T-103$ & 58 & 59 & 27 & 17 & \\
\hline 58 & $T-104$ & 62 & O/s & 445 & 169 & All TCs OS, work order issued \\
\hline 59 & $T-105$ & Q/s & ors & 98 & 43 & (2) No TC tree per Riser Configuration document \\
\hline 60 & $T-106$ & 58 & $\mathbf{5 9}$ & 21 & 15 & \\
\hline \multirow[t]{2}{*}{61} & $T-108$ & Os & Ors & 180 & 73 & All TCs O/S, TC tree laying on ground, J-3 issued 1/92 \\
\hline & & & & & & and again 7/83, last reading $63 \mathrm{~F}$. In 11/91 \\
\hline 62 & $T-109$ & Ors & Ors & 58 & 29 & All TCs O/S, work order lssued, last reading 75 F. in $2 / 91$ \\
\hline 63 & $T-111$ & 62 & 59 & 458 & 174 & TCS $\$ 1,2,5,60 / \mathrm{s}$ \\
\hline 64 & $T-112$ & 62 & 58 & 67 & 32 & \\
\hline 65 & $T-201$ & 61 & 59 & 29 & 150 & \\
\hline 66 & $T-202$ & 61 & 58 & 21 & 110 & \\
\hline 67 & $T-203$ & 61 & 75 & 35 & 182 & \\
\hline 68 & $T-204$ & 62 & 60 & 38 & 197 & \\
\hline
\end{tabular}




\title{
TABLE A-6. NON-WATCH LIST LOW HEAT LOAD TANKS $(<40,000$ Btu/h)
}

\author{
(Page 3 of 3)
}

Temperatures are taken semiannually in January and July, unless otherwise indicated, in the following 96 single-shell tanks. Legend follows table.

\section{Highest Temperatures} taken in waste Total Waste (1)

Tank No. Jan. 93 Jul. 93 Kgal Inches

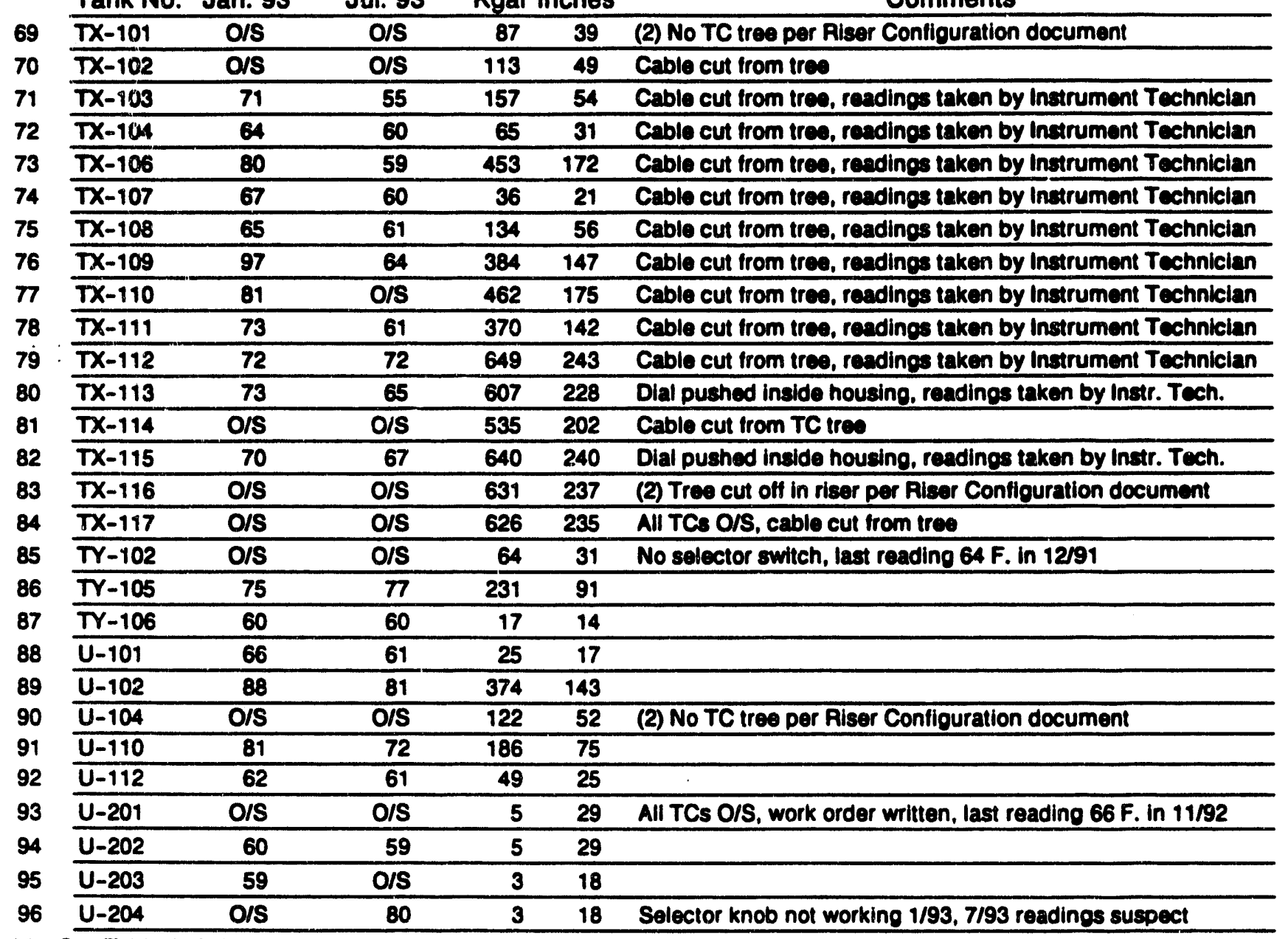

(1) See Table A-2 (footnote 6 ) for waste gallons/inches calculations.

(2) Thermocouples in nine tanks (BX-104, BY-102, BY-109, SX-115, T-102, T-105, TX-101, TX-116, and U-104) are out of service due to no TC trees in these tanks, or the thermocouples have been cut off, covered over, or are otherwise unable to function. per the Riser Configuration document. (Also see comment section above)

\section{Yestis:}

TC - Thermocouple

TMACS - Tank Monitor \& Control System

O/S - Out of service

Riser Configuration document - WHC-SD-RE-TI-053, REV 8, "Riser Configuration Document for Single-Shell Tanks," September 1991

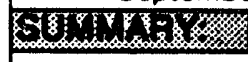

Readings obtained in July 1993 Tanks

No readings (TC trees O/S - includes nine tanks with no trees - see footnote above) Total low heat load tanks 


\section{TABLE A-7. SINGLE-SHELL TANKS MONITORING COMPLIANCE STATUS 149 TANKS (Sheet 1 of 5)}

The following table indicates whether Single-Shell tank monitoring was in compliance with the requirements as spectied in the applicable documents as of the last day of the applicable month:

NOTE:

All Watch List and High Heat tank temperature monttoring is in compliance. (5)

All Dome Elevation Survey monitoring is in compllance.

All Drywell monitoring is in compllance.

Psychrometrics (2)

In-tank Photooraphs (3)

Presesure Monttoring(6)

CAMVAMP Montioring (7)

Vapor Monltoring (8)

Information as of 12/31/93

\begin{tabular}{|c|c|c|c|c|c|c|c|c|c|}
\hline \multirow[b]{3}{*}{$\begin{array}{l}\text { Tank } \\
\text { Number }\end{array}$} & \multirow{2}{*}{\multicolumn{2}{|c|}{ Cotegory }} & \multirow{3}{*}{$\begin{array}{c}\text { Tempereture } \\
\text { Rasdines } \\
\text { (O)(0) }\end{array}$} & \multirow{3}{*}{\multicolumn{2}{|c|}{$\begin{array}{l}\text { Surface Lewl } \\
\text { Raendinge: (1) } \\
\text { (-857) }\end{array}$}} & \multirow{3}{*}{$\begin{array}{c}\text { Low } \\
\text { Randinge } \\
(-357)(9) \\
\text { isirion }\end{array}$} & \multicolumn{2}{|c|}{ Redietion Rasdinos } & \multirow{3}{*}{$\begin{array}{l}\text { Dome } \\
\text { Elevation } \\
\text { Surveys } \\
\text { (OSRYsAr) }\end{array}$} \\
\hline & & & & & & & Leteral & Drymell & \\
\hline & $\begin{array}{l}\text { Watch } \\
\text { Lat(6) }\end{array}$ & $\begin{array}{l}\text { High } \\
\text { Heat }\end{array}$ & & & & & $\begin{array}{l}\text { Randing } \\
\text { (-357) }\end{array}$ & $\begin{array}{l}\text { Randings } \\
\text { (OSASAR) }\end{array}$ & \\
\hline$A-101$ & $x$ & & & & Kns & & orc & y & 恼 \\
\hline A-102 & & & & xy, & 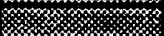 & 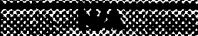 & ore & x & \\
\hline A-103 & & & 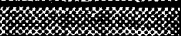 & 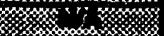 & 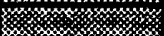 & 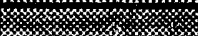 & ac & & \\
\hline A-104 & & $\bar{x}$ & 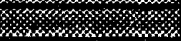 & 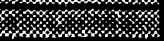 & 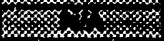 & Kk & ort & 纱 & \\
\hline A-105 & & $\bar{x}$ & 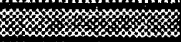 & 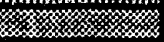 & 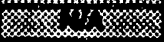 & ${ }^{\prime} x_{2}^{\prime}$ & 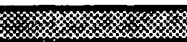 & & \\
\hline$A-100$ & & & 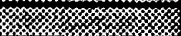 & $x^{2}$ & 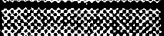 & 婉纹 & $x_{x}$ & & \\
\hline$\sqrt{x-101}$ & $\bar{x}$ & & 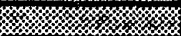 & 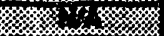 & 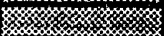 & 沙 & কে & 1. & \\
\hline$\sqrt{x-102}$ & & & 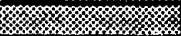 & 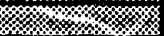 & s & 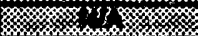 & x媇 & & \\
\hline AX-103 & $\bar{x}$ & & & s. & 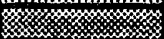 & 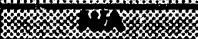 & / & & \\
\hline$a x-104$ & & & 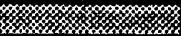 & & 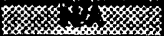 & 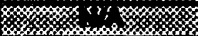 & x & & \\
\hline B-101 & & & 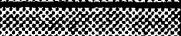 & 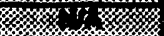 & 桨 & 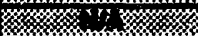 & k k $x$ k & & \\
\hline$B-102$ & & & 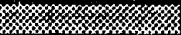 & xy & 玹空 & 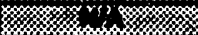 & 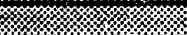 & & \\
\hline$B-103$ & $\bar{x}$ & & 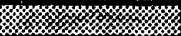 & xys & & 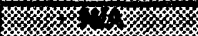 & 14 & & \\
\hline$B-104$ & & & 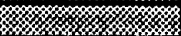 & 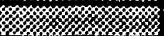 & 桨 & & 将 & & \\
\hline$B-105$ & & & 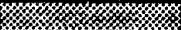 & 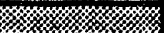 & 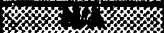 & 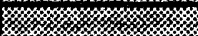 & צ' & & \\
\hline $8-100$ & & & & 纵 & xym & 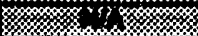 & & & \\
\hline$B-107$ & & & 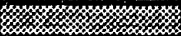 & & 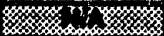 & 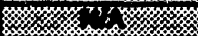 & V & & \\
\hline $8-103$ & & & 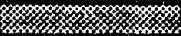 & 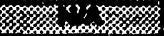 & 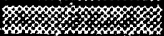 & ${ }^{\prime} x$ & & & \\
\hline $8-100$ & & & 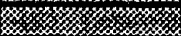 & 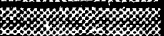 & 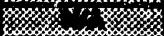 & 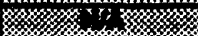 & א. & 侄 & $8 \%$ ry \\
\hline $8-110$ & & & 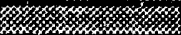 & 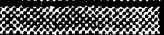 & 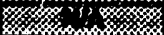 & 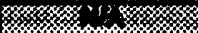 & 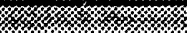 & & \\
\hline$B-111$ & & & & \% & 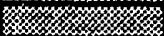 & 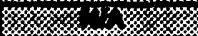 & 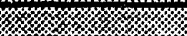 & & \\
\hline$B-112$ & & & & 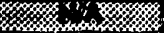 & 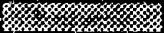 & skx & 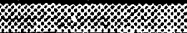 & & \\
\hline B-201 & & & 紧纹 & 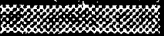 & 森兹 & 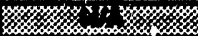 & 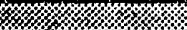 & & \\
\hline$B-202$ & & & 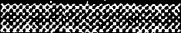 & 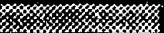 & 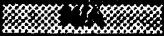 & 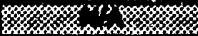 & & & \\
\hline$B-203$ & & & 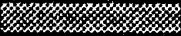 & 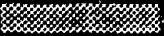 & 媇 & 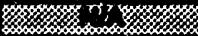 & 率 & & \\
\hline$B-204$ & & & & 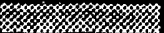 & 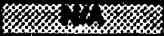 & 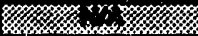 & 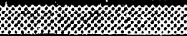 & & \\
\hline$B X-101$ & & & OC & 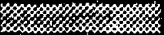 & 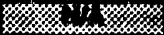 & \% & 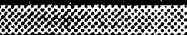 & & \\
\hline $8 x-102$ & $x$ & & & 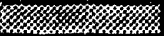 & rsck & 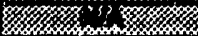 & & & \\
\hline $8 x-103$ & & & Ore & 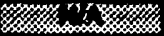 & 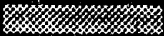 & 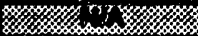 & 桨 & & \\
\hline BX-104 & & & OE-OC & sxy, & 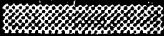 & 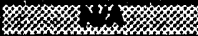 & & 誃㡅 & \\
\hline $8 x-106$ & & & 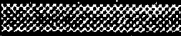 & 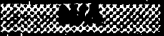 & 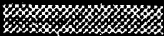 & 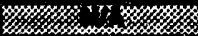 & 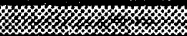 & / / & \\
\hline $8 x-100$ & $\mathbf{x}$ & & 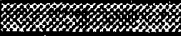 & 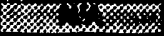 & 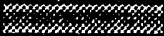 & 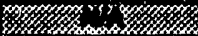 & 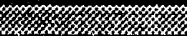 & & \\
\hline $8 x-107$ & & & oc & 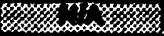 & 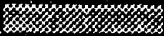 & 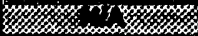 & & & \\
\hline
\end{tabular}

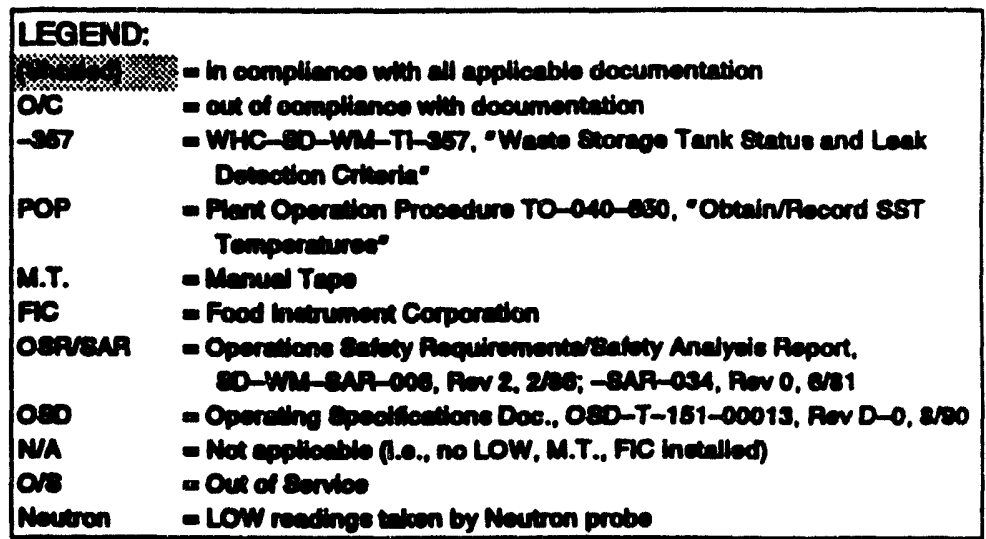




\section{TABLE A-7. SINGLE-SHELL TANKS MONTTORING COMPLIANCE STATUS 149 TANKS (Sheet 2 of 5)}

Information as of 12/31/93

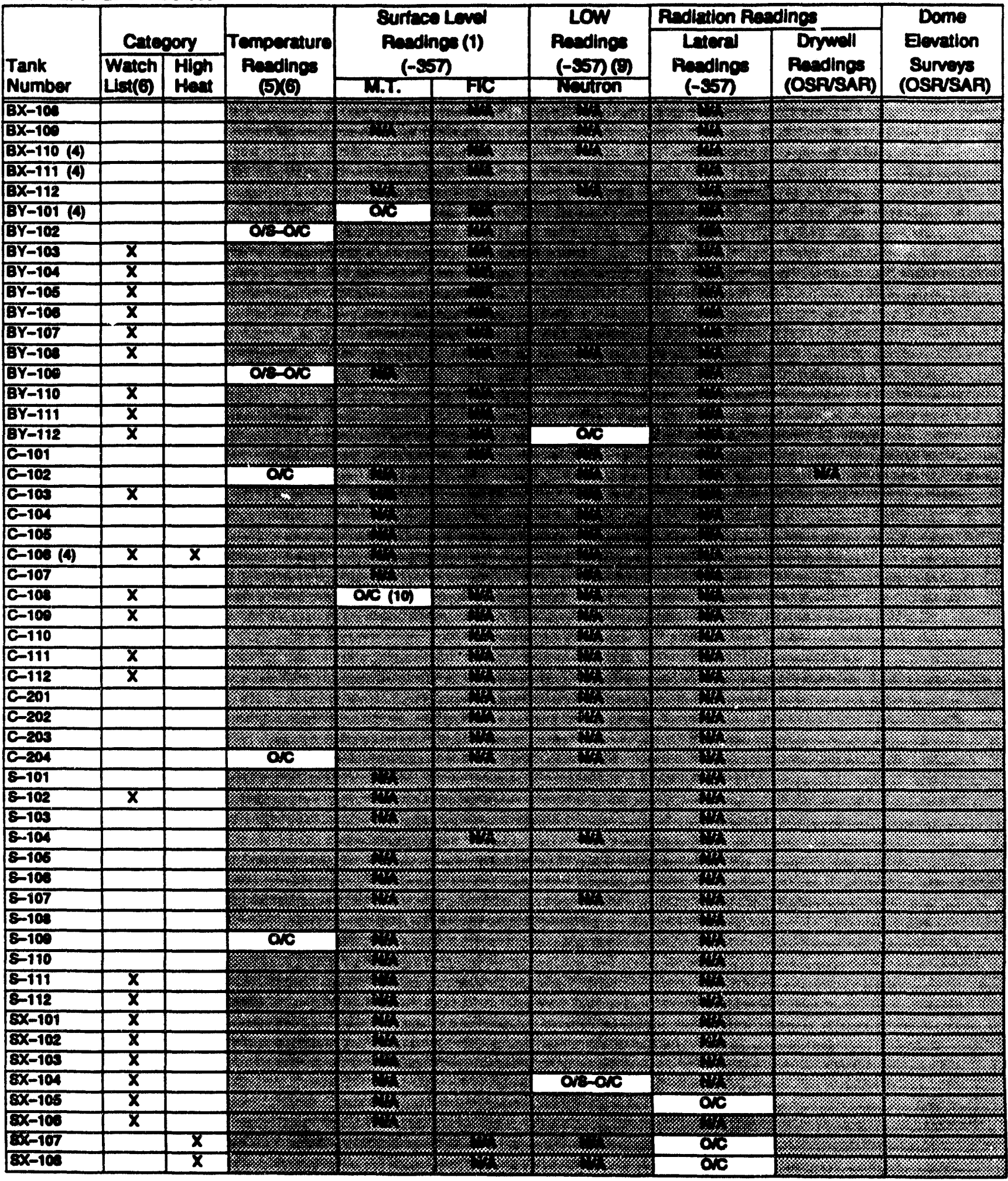


WHC-EP-0182-69

\section{TABLE A-7. SINGLE-SHEL TANKS MONTTORING COMPLIANCE STATUS 149 TANKS (Sheet 3 of 5)}

Information as of 12/31/8s

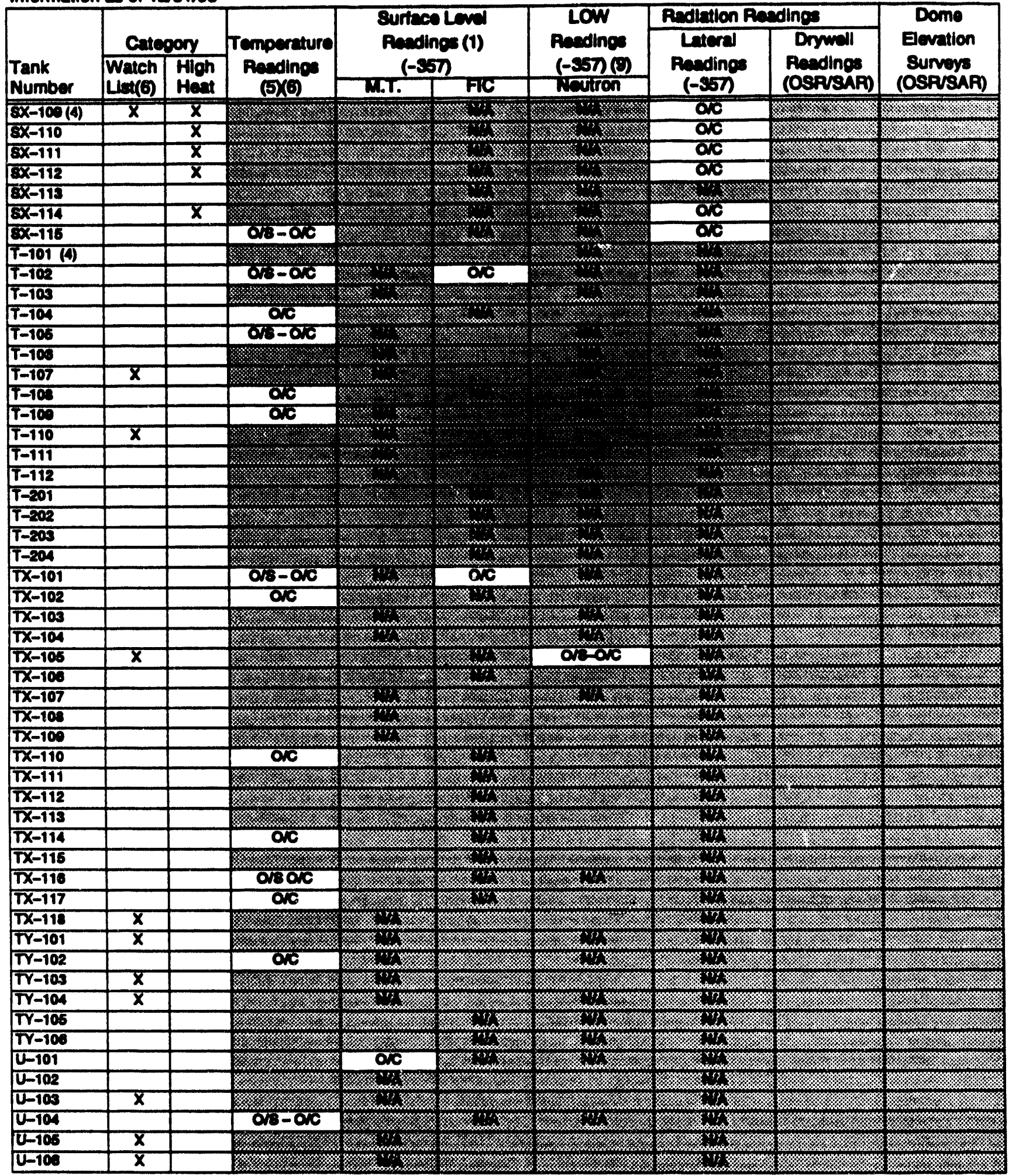




\section{TABLE A-7. SINGLE-SHEL TANKS MONTTORING COMPLIANCE STATUS 149 TANKS (Bheet 4 of 5)}

Information as of 12/31/03

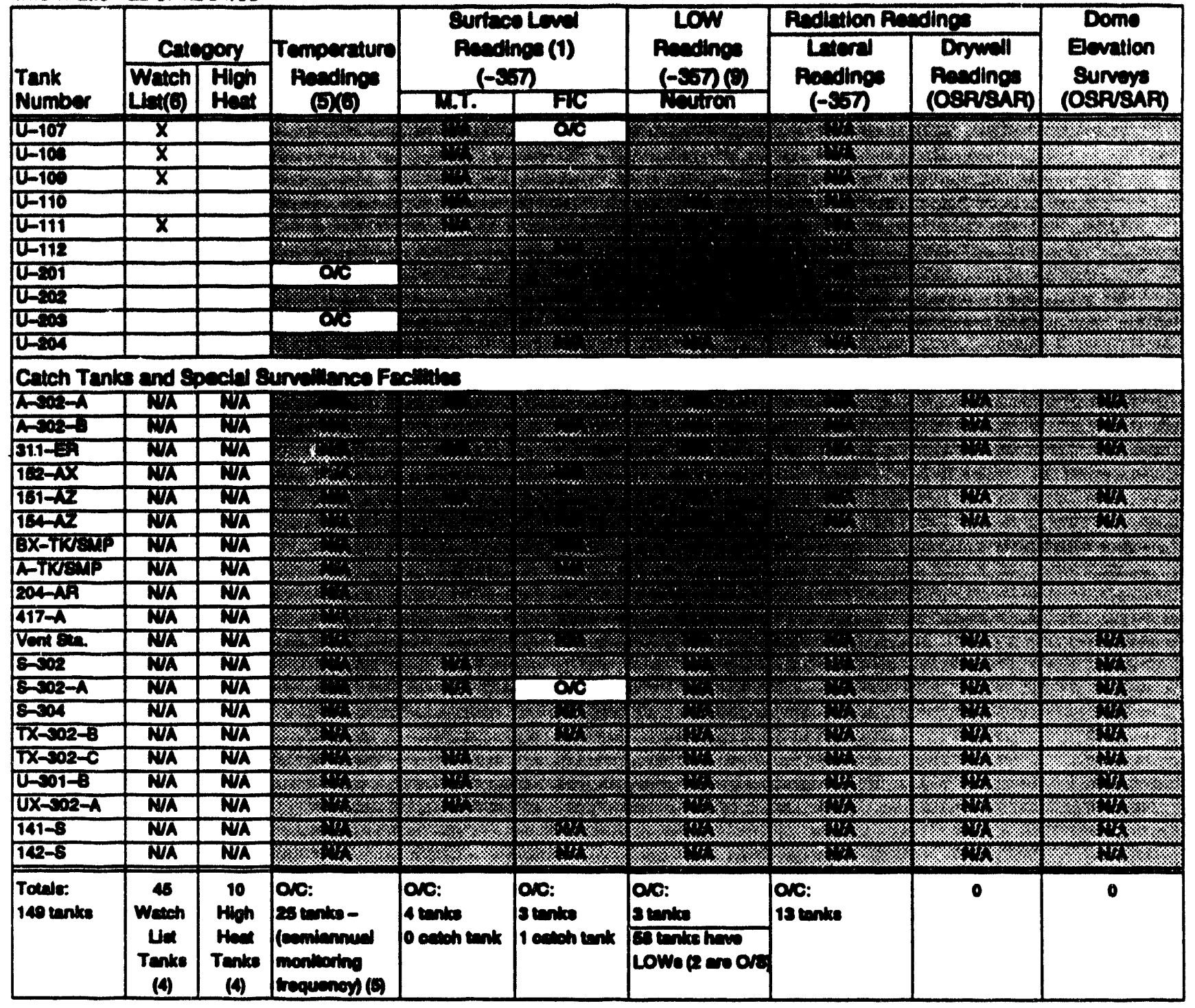

See Footnotes on next page 
MHC-EP-0182-69

\section{TABLE A-7. 8INGLE-SHELL TANK8 MONITORINO COMPLIANCE STATUS 149 TANKS (Sheet 5 of 5)}

\section{Footnotes:}

1. All sste have oither manul tape or Fic, with the exeeption of 8-108 and T-101, wich have both. Tenk 101-T also has zip cord. All sit Fics are cemeted to Cass, with the exception of ex-106; however, the comnetion for mery tenks is broken. For such cases, canusl readings ore taken. Manual surface leval readings include readinge taken by manul tape, manual pic (not connected to

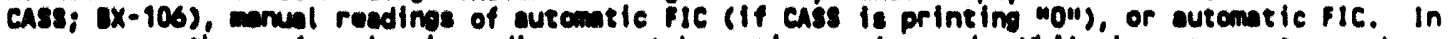
som cases, the surface level readings ore taken wing a sip cord. While leas accurate, such readings are sccepteble for meeting the surface level reading requircenents.

2. Migh heet tonks have ective exhowatera; peychroantrice are taken in these tanks (A-106/105, c-105/106 loffective July 1, 1993, C-105 is no lonear a high heat load tanks, sx-107, 108, 109, 110, 111, 112, and 114). The exheusters on A-104/105 have been doun since octobor 1991; no reedinges are being taken. Peyehrametric readinge have not been taken in the $8 x$ high hest loed tanke since July 1993. The trequency of poyehrometric reedines in ssts is duternined oy the coenizent Enoineers for

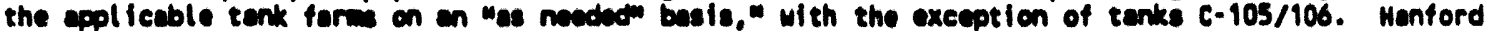
Federal Fecillity Agrewent and Coneent Ordar." Machingten state Departinent of Ecology, U. S. Emironemtal Protection Ageney, and U. 8. Departwent of Enorgy, 1992 (Iri-Party Agreement) requires paychromentic readings to be taken in C-105/106 on o monthly frequency. Last roedings ware taken Detober 27, 1993; readinge were not taken In Moveber. A Oiserepency Report was lssuad. Readings were not taken in December.

3. In-tank photographs are requeted on en mas needede basis. No in-tenk photographs wore taken batween september 1990 and March 1993.

4. Two tanks are on both catesory llsts (C-106 and 8x-109). In July 1993, C-105 was ramovad from the

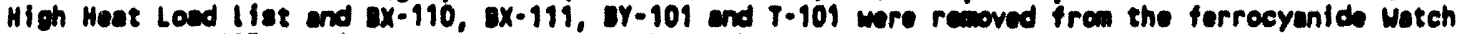
List. Only C-10S continues to be taken weekly; the other tenks are now taken semiannially. U-111 was added to the Organic sal ts list Auvust 31, 1993.

5. Teaperature readings may be regulated by $000,-357$, or P0p. Addlitionally, high hest load tanks are regulated by 0sk/sAk. Thermocouples in the nine tenks desienated 0/3-0/C are out of service; there ore either no thermocouple trees in these tenks, or trees have been cut off, covered over, or ore otherwise unable to function. The oso does not requile readinge or repoir of out-of survice therwocouples for the 9 low heat load $(440,000$ itwh) tenks. However, the pop requires that ot tempts are to be mode semlenmully in Jemuary and July to obtain reedings for these tanks. Temperatures were taken in July 1993; - total of 25 tanks are 0/C because either no attempt to obtain the temperature was mads, or : reading could not be obtained.

6. WSafoty Measures for Waste Tanks at Menford Muclear Reservation Section 3137 of the Mational Defense Authorization Act for Fiscal Year 1991, "Novewbar 5, 19\%0, Publ le Law 101-310, (the Muyden Amendanent") requires continuous pressure monitoring and temperature monitoring in Watch List tanks. WHC-EP-0422 REV 1, WA PIan to Implewent Remediation of Waste Tank safety lssues ot the Hanford Site," December 1991, addresses these monitoring issues. WHC-EP-0600, "Status Report on Resolution of Waste Tank Safoty lesues at the Hanford site," lesued August 1993, deseribes the resolution strategy for these safety issues.

7. Continuous Air Monitoring (CAM) compliance and Rediation Area Monitoring Panel (RAMP) compliance are not addressed in this table.

8. Double-shell tank farm sy has the only tanks with continuous vapor/flemable ges monitoring; not addressed in this table.

9. An Engineering Change Notice was issued November 1993, changing the monitoring frequency of Low readinge by Gemm probe to be taken wby request only." The Gewes probe column has therefore been deleted.

10. Surface level meseurcment monitoring is out of compliance in C-108 bacause the manual tape was removed to install a thermocouple tree. This tank does not have an FIC. A work package is pending to re-instell the manual tape. 


\section{TABLE A-8. DOUBLE-8HEL TANKS MONTOANG COMPLLANCE STATUS 28 TANK8 (Bhaet 1 of 2 )}

The following table inclacates whether Double-encil bank monitoring wes in compliances with the

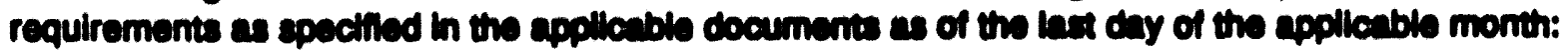

More:

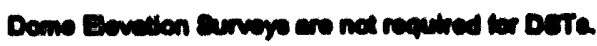

Promremetibe (19)

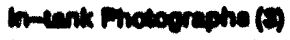

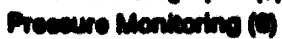

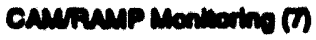

Veper Mentoring (i)

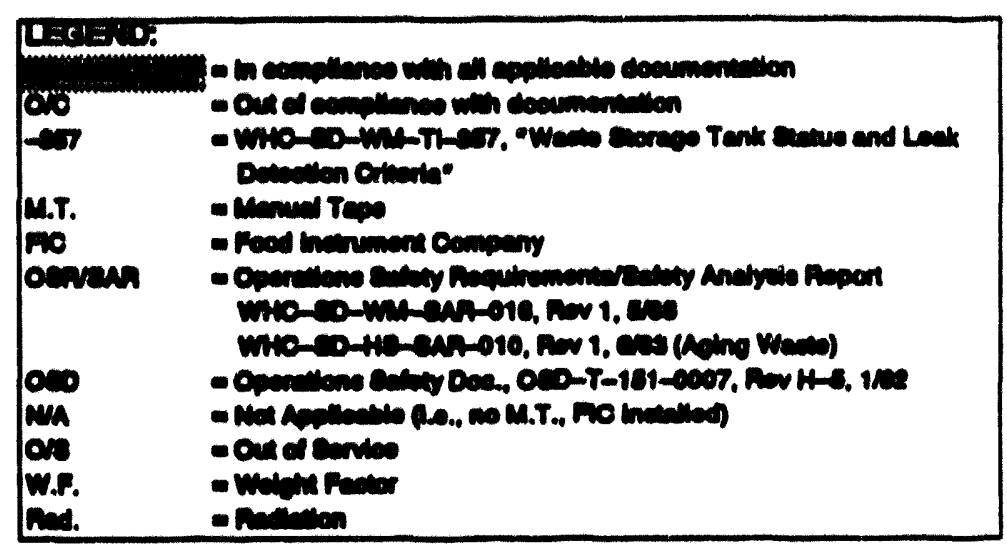

Intormation es of 1201/is

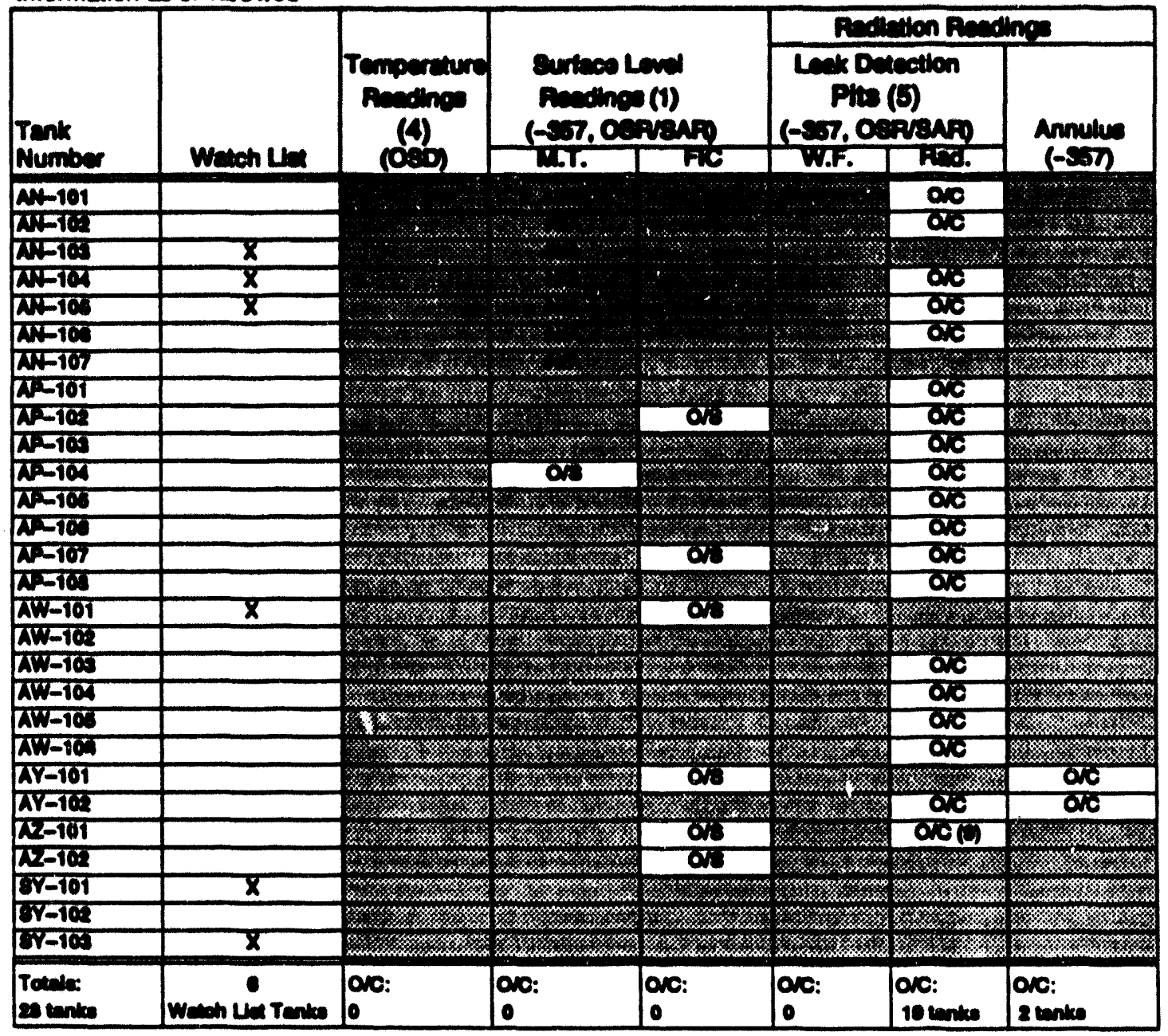

See footnotes next paga: 
TABLE A-8. DOUBLE-SHELL TANKS MONITORINO COMPLIANCE STATUS 28 TANKS (Sheet 2 of 2 )

\section{Footnotes:}

1. All Dste hove both amul tepe and PIC, with the exception of the AN Tenk Parm which has only fics. The eanul tepe is wed men the PIC is out of earvice. O/C will be thown witen no readings are obtalned.

2. Payehrometric readinge are only taken on tenks with ective oxhoustora; oll Dsts hove oetive exhaweers. The frequency of payehreantric readings in oste is deterinined by the cognizant

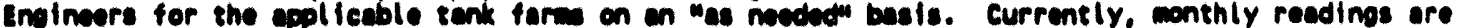

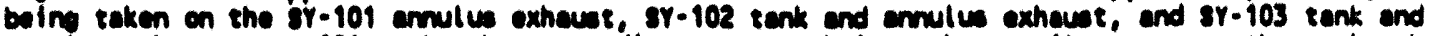
emulus exheust. or-101 tenk exhoust reedings ore not boing taken intll a port on the tenk exhaust heeder becence ovalieble for exheuster readings. No other paychramerle readines are currently boling taken monthly.

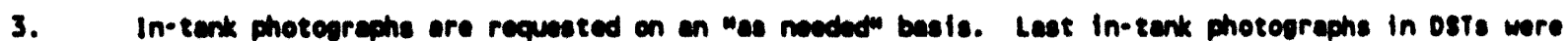
token in April 19e0.

4. OSp spaclftes ost tcmporature linits, orediente, ote. Tenk sr-101 tcmperatures are obteined enlftwise with inereased readinge taken prior to and following gas venting.

5. Pollure of both leak detection syetem requires repoir of ot least on ovetem within 5 working doys. Fallure of on syete only, repalr mest be within 10 workdays par -337 doevent. If the polr of

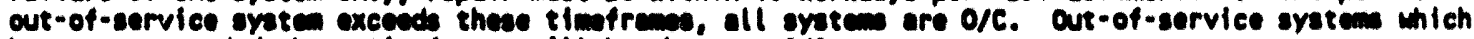
have not exceeded these timetremes will be thein as o/s.

6. ugafoty Mesoures for Waste Tenks at Manford Muclear Regervation, section 3137 of the Mational

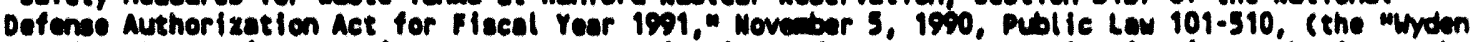

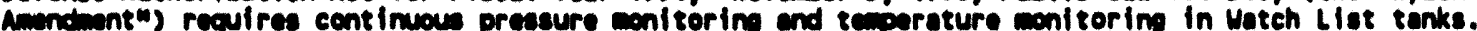

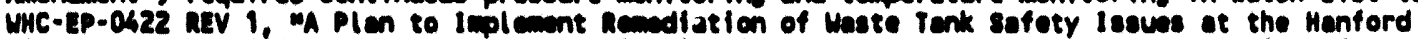
site," December 1991 , coddresese these monitoring issues. A status report on resolut ion of Waste Tenk safety Iesues of the Menford si te has been prepered but has not yet been cleared for public roleasi.

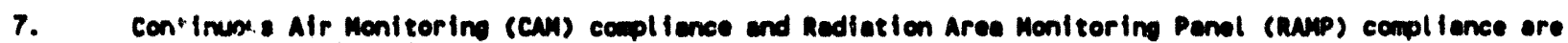
not acodresesed in this table.

8. Double-shall tank form sy has the only tenks with contimuous veporfflemable gas monitoring; not addreseed in this table.

9. Although date are boing recelved for N2-101, this information is considared questionable. 


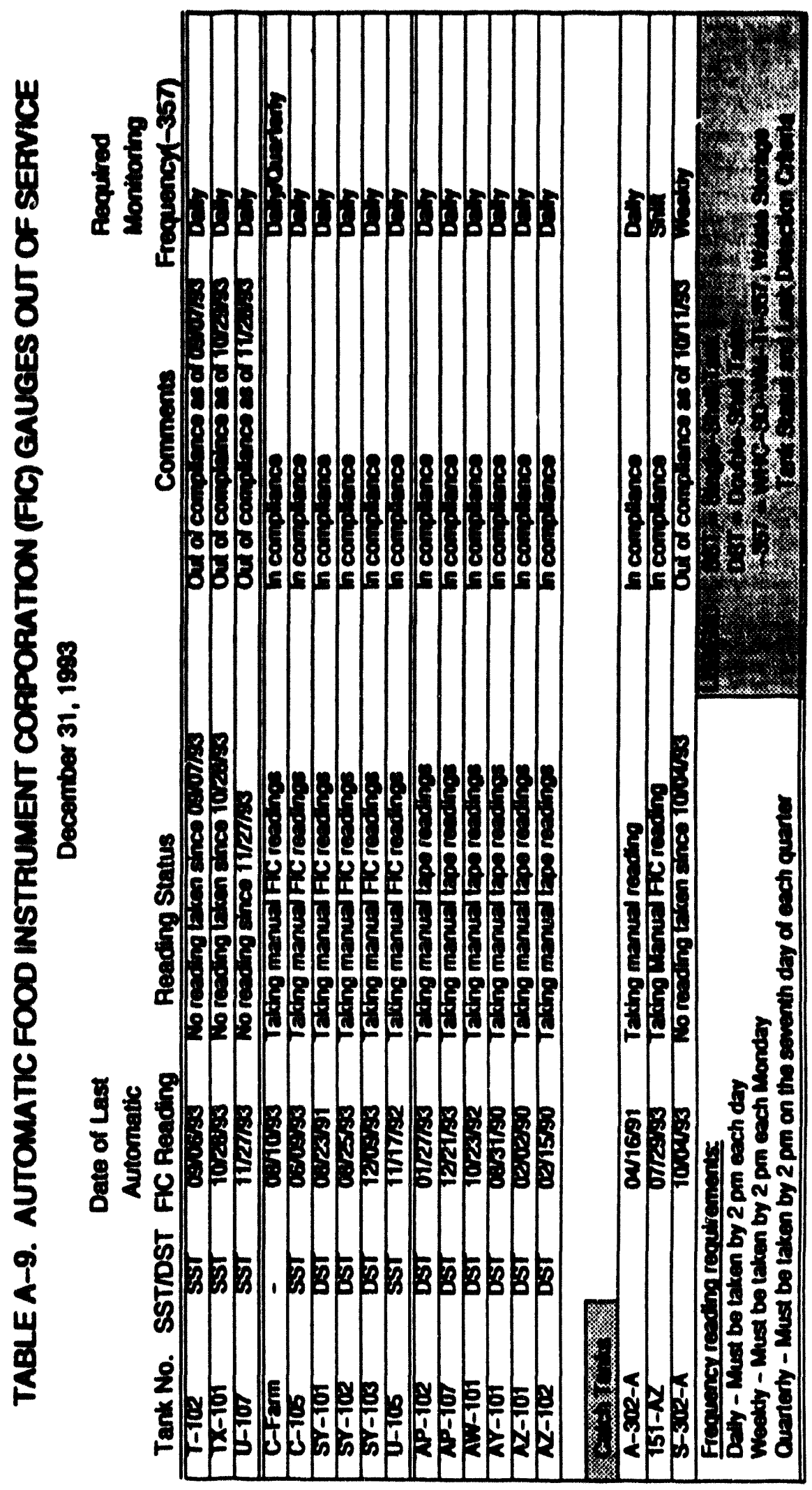




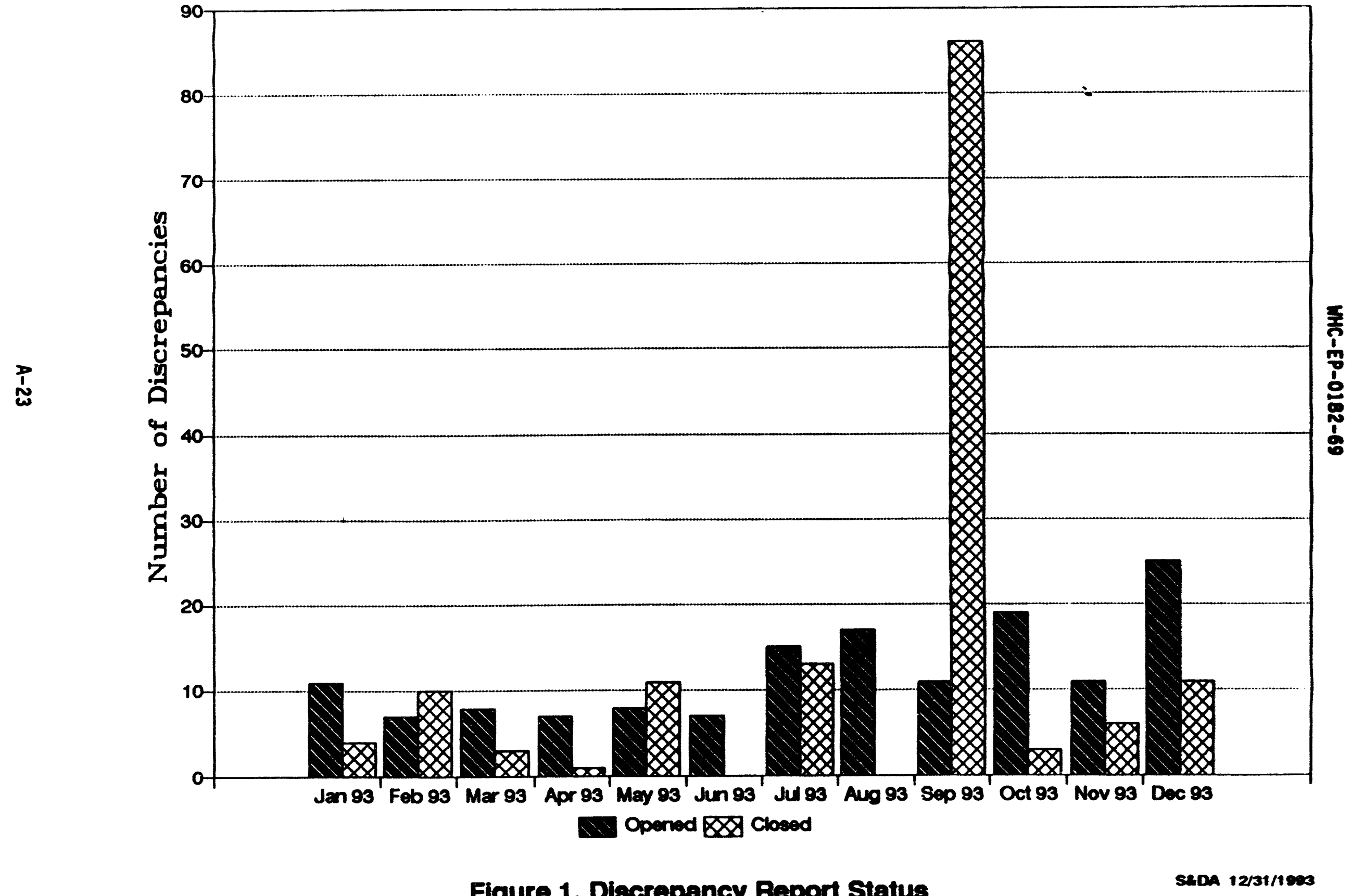

Figure 1. Discrepancy Report Status

sod 12/31/10e3 


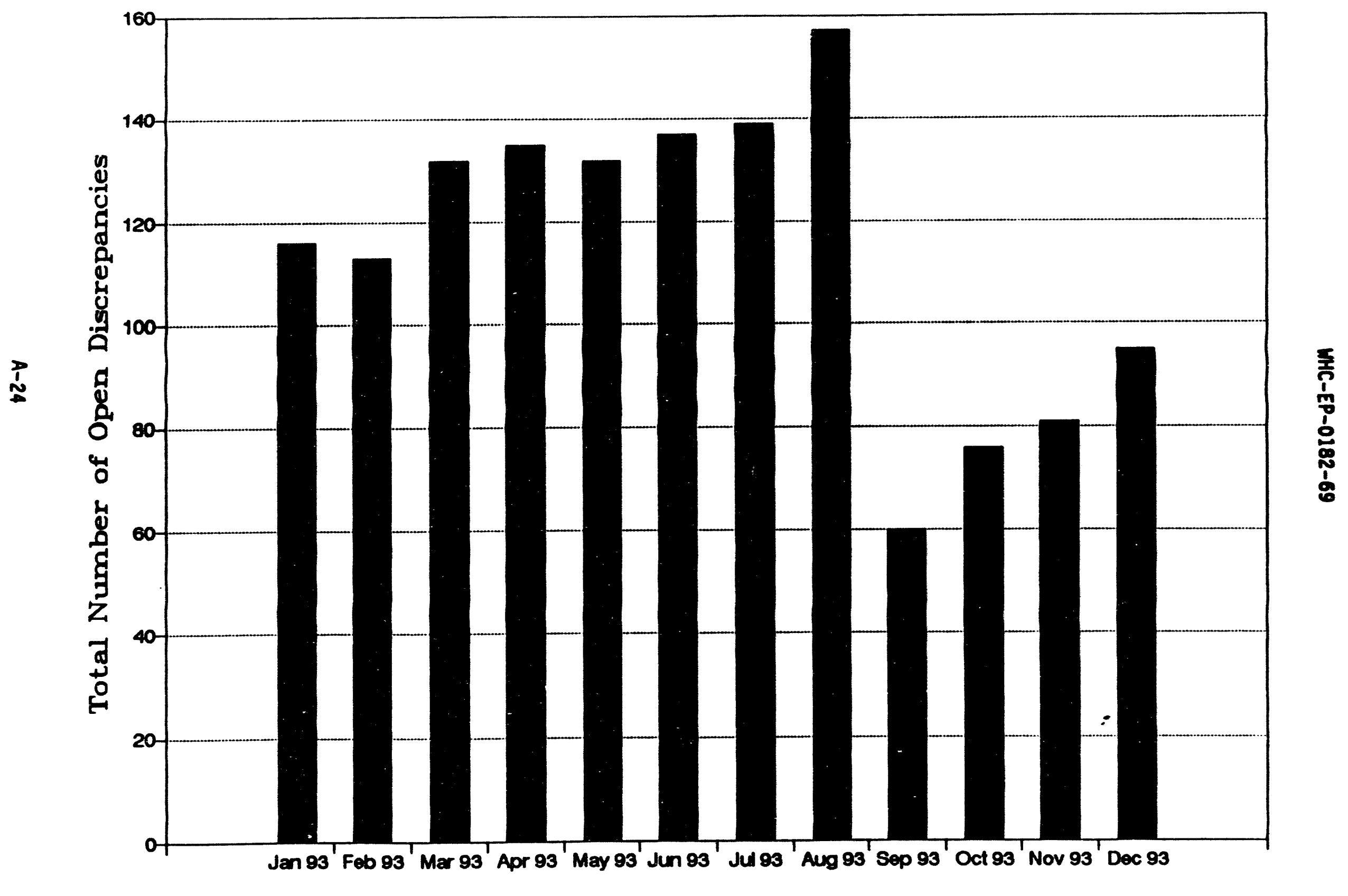

Figure 2. Discrepancy Report Cumulative Total

SeDA $12 / 31 / 1003$ 
Number of Open Discrepancies

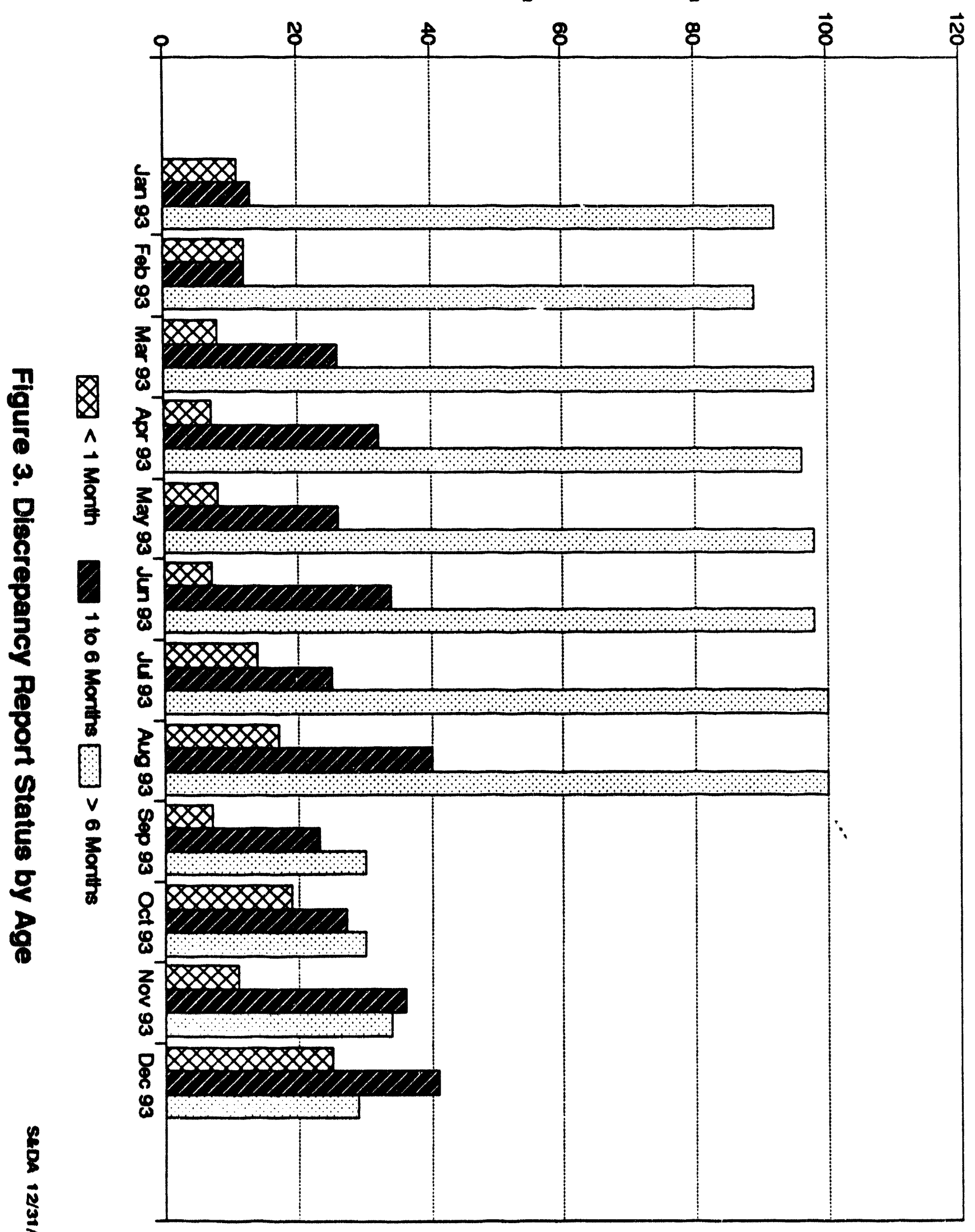


MHC-EP-0182-69

This page intentionally left blank 
WHC-EP-0182-69

\section{APPENDIX B}

\section{DOUBLE SHELL TANK WASTE TYPE AND SPACE ALLOCATION}


WHC-EP-0182-69

This page intentionally left blank 
TABLE B-1. DOUBLE-SIELL TANK WASTE TYPE AND SPACE ALLOCATION DECEMBER 1993

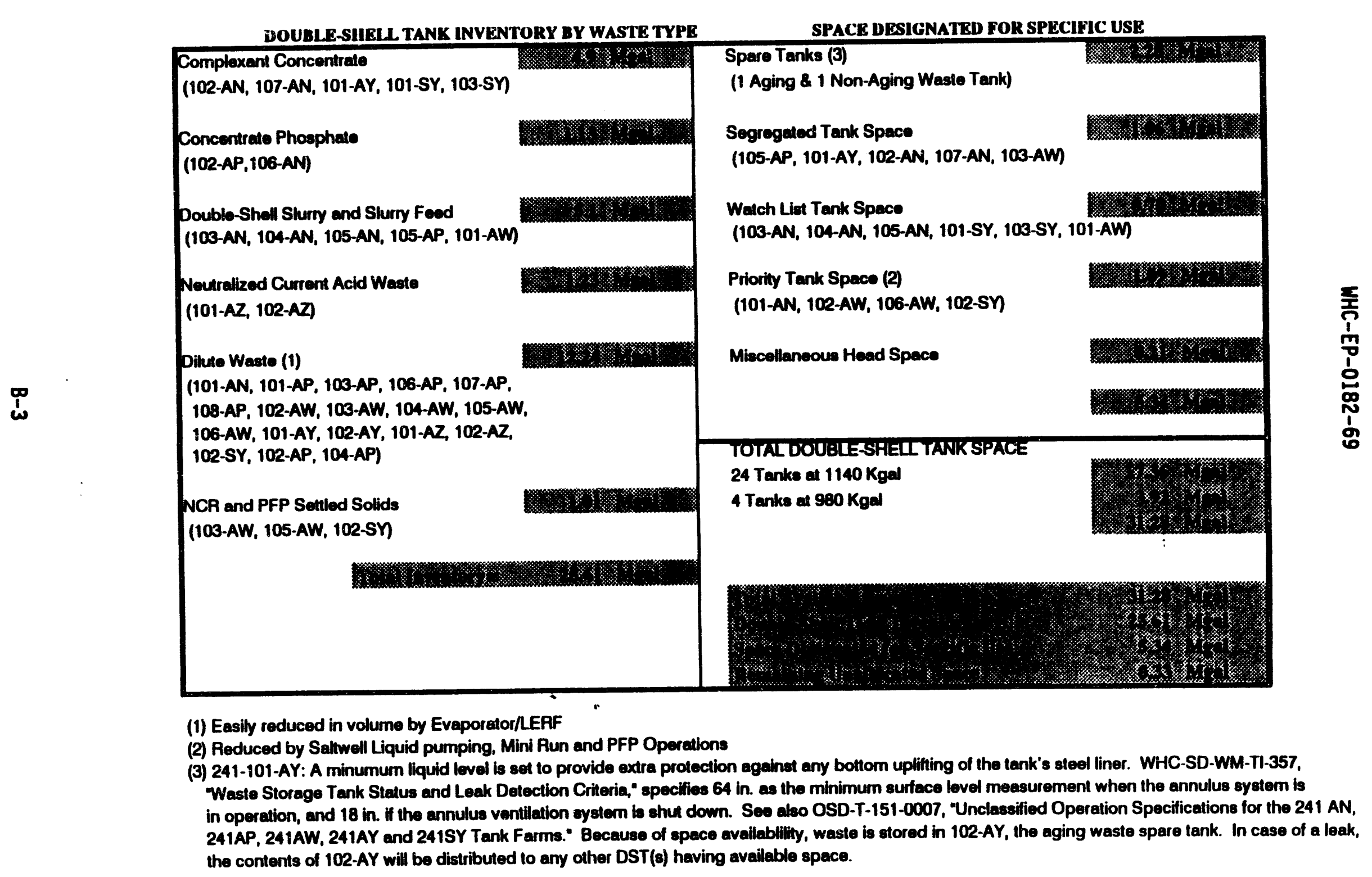

Note: Net change in DSTs since last month: $91 \mathrm{Kgal}$. 


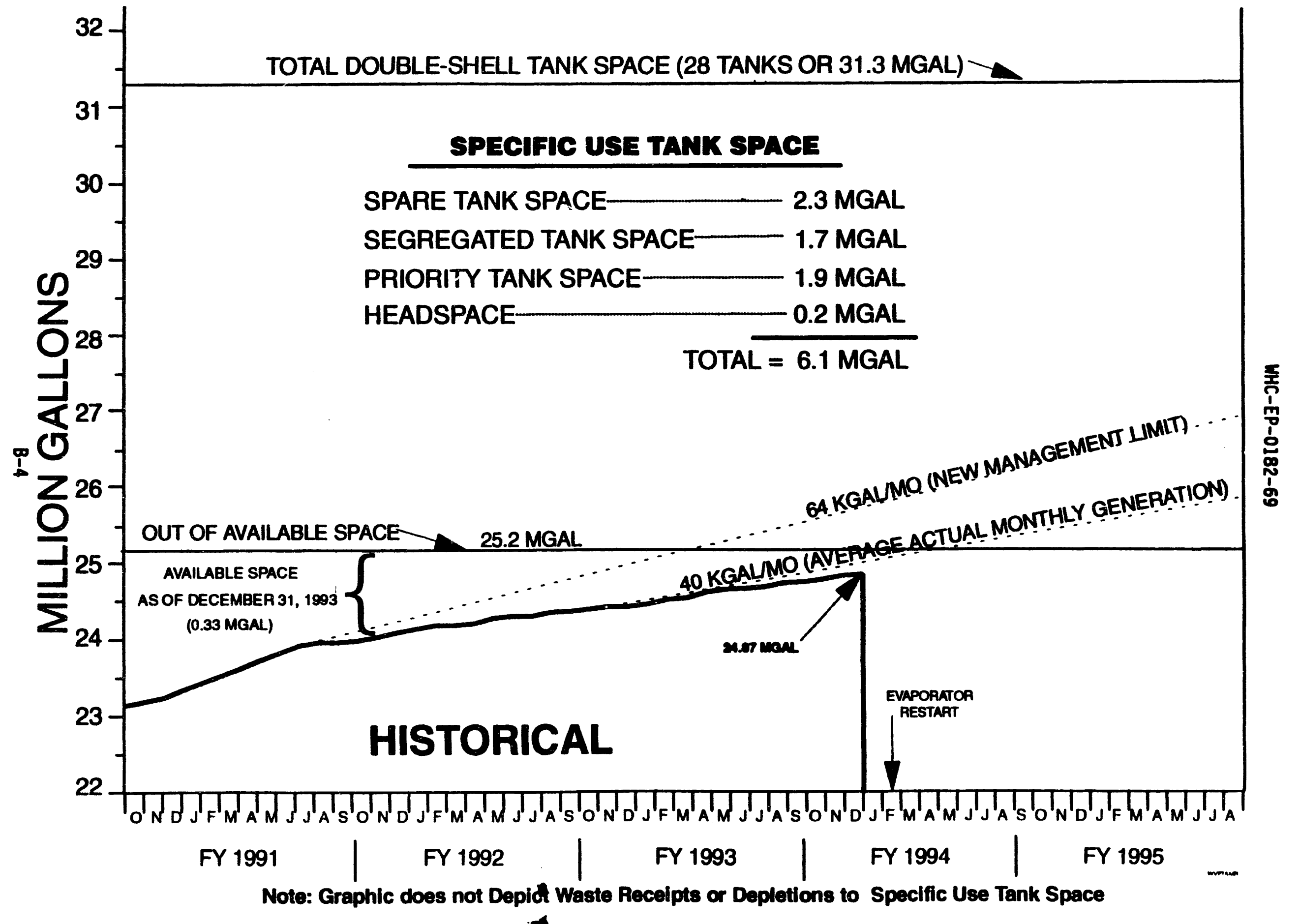

FIGURE B-1. CURRENT STATUS AND CONTINGENCY SPACE FOR THE 242-A EVAPORATOR RESTART 


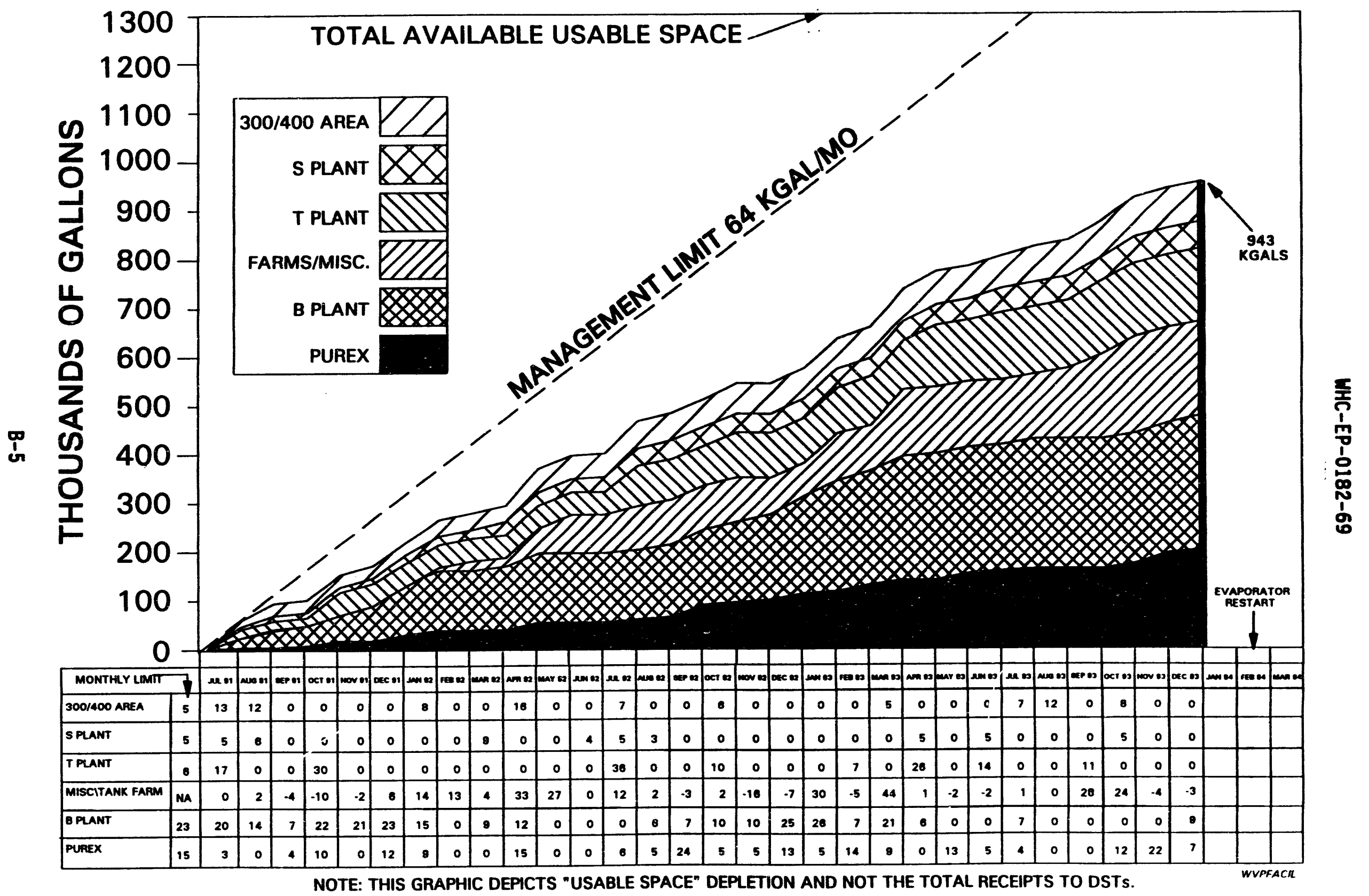

FIGURE B-2. TOTAL AVAILABLE USABLE TANK SPACE 


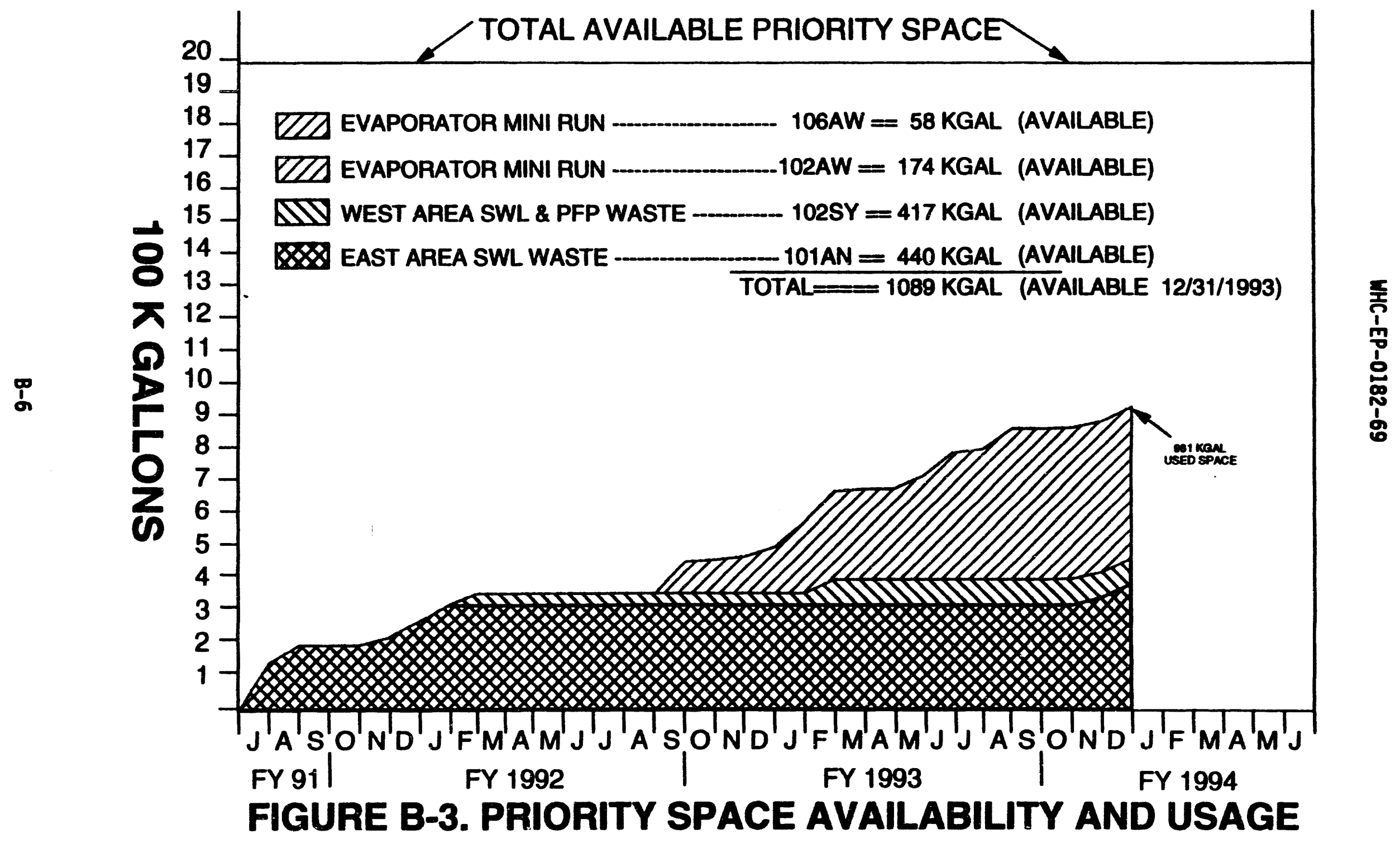




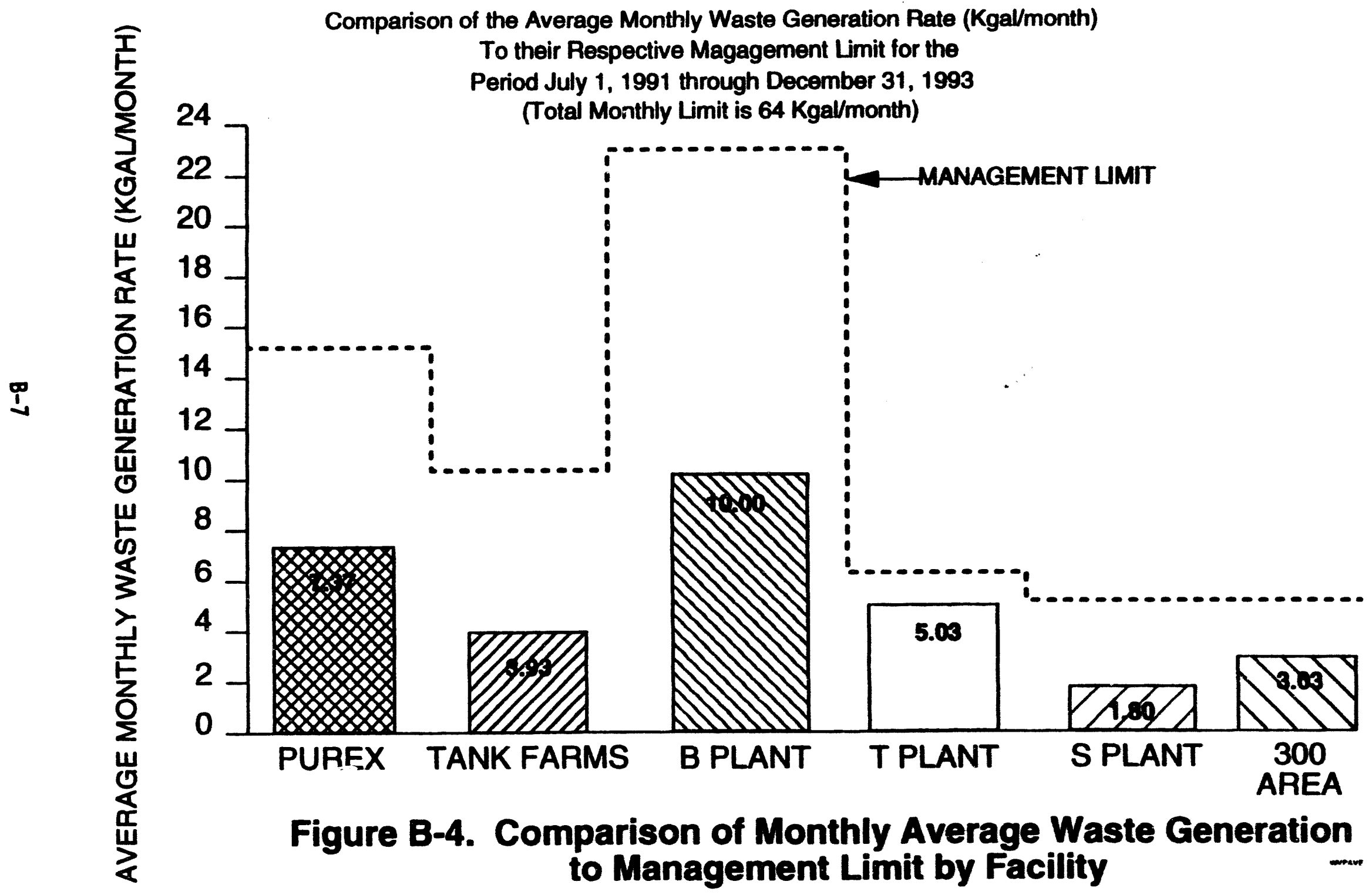




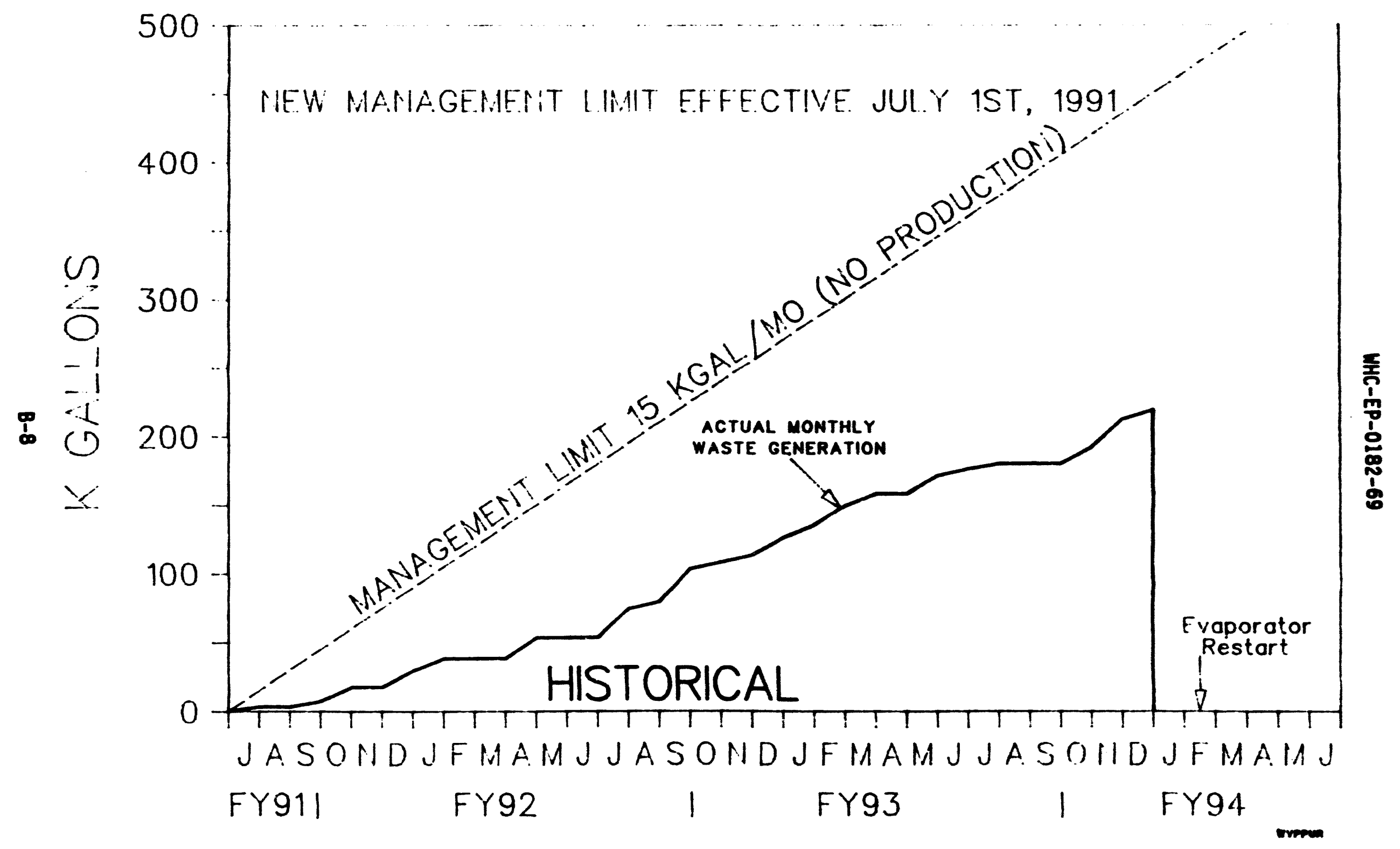

FIGURE B-5. PUREX MONTHLY WASTE GENERATIONS 


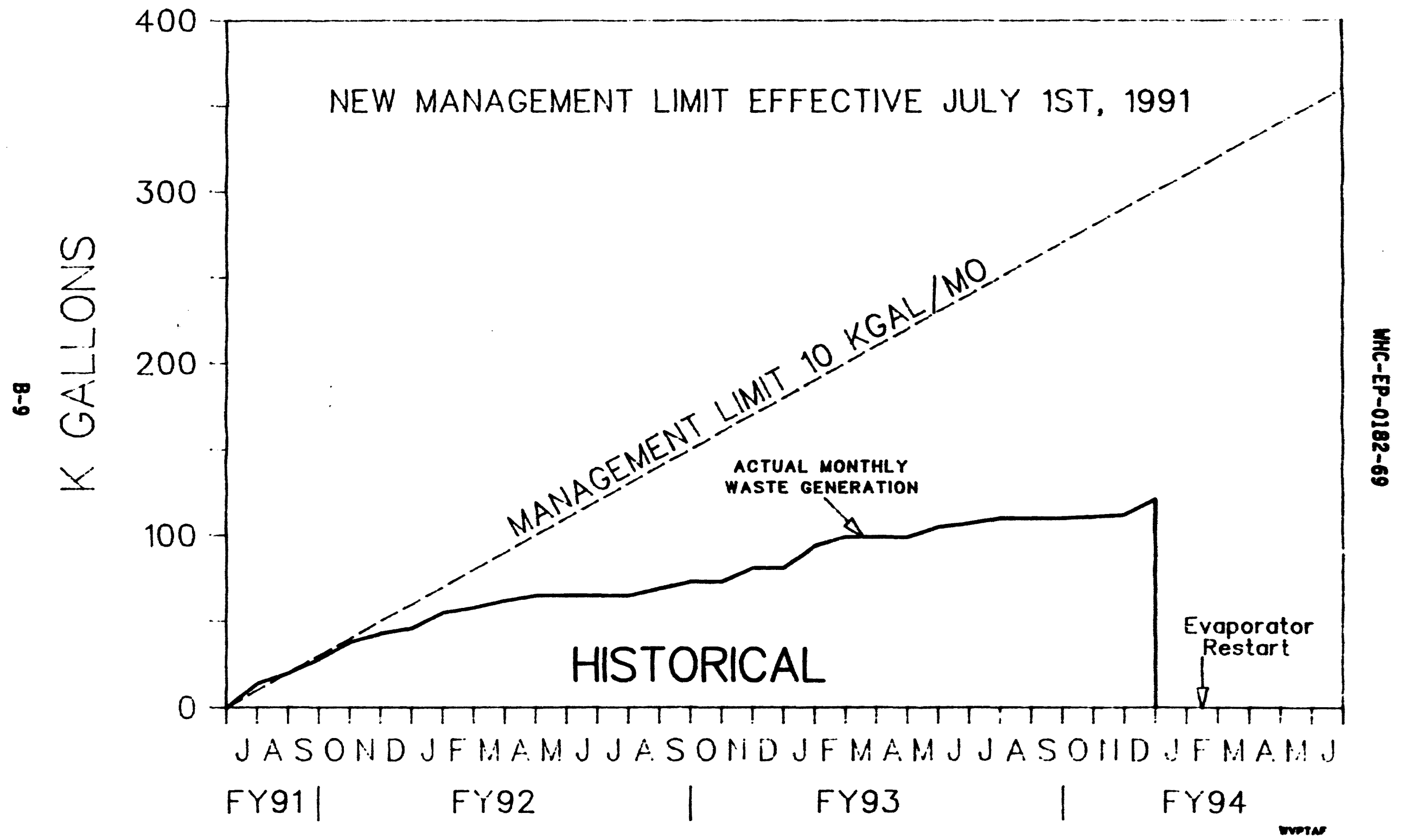

FIGURE B-6. TANK FARM MONTHLY WASTE GENERATIONS 


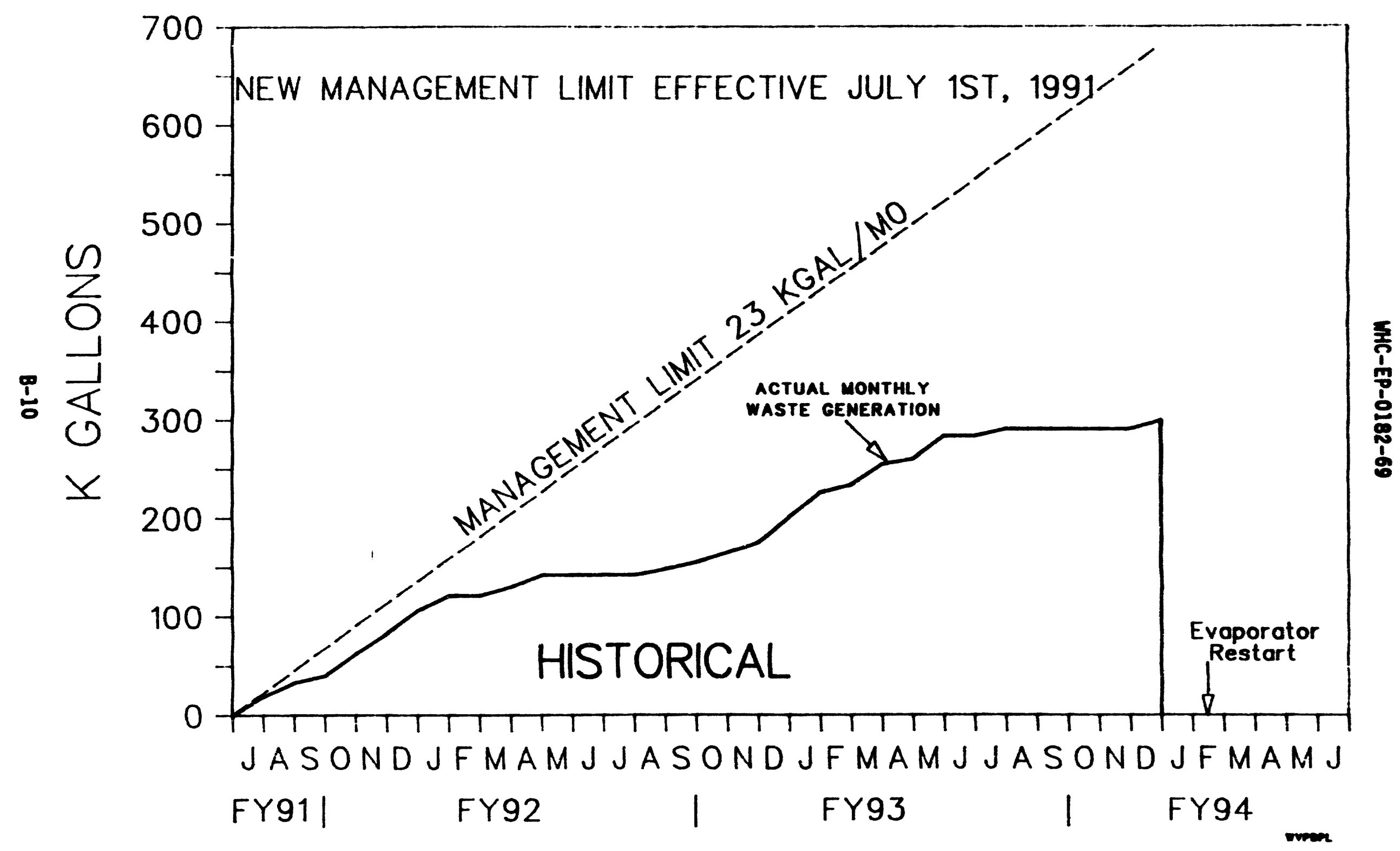

FIGURE B-7. B PLANT MONTHLY WASTE GENERATIONS 


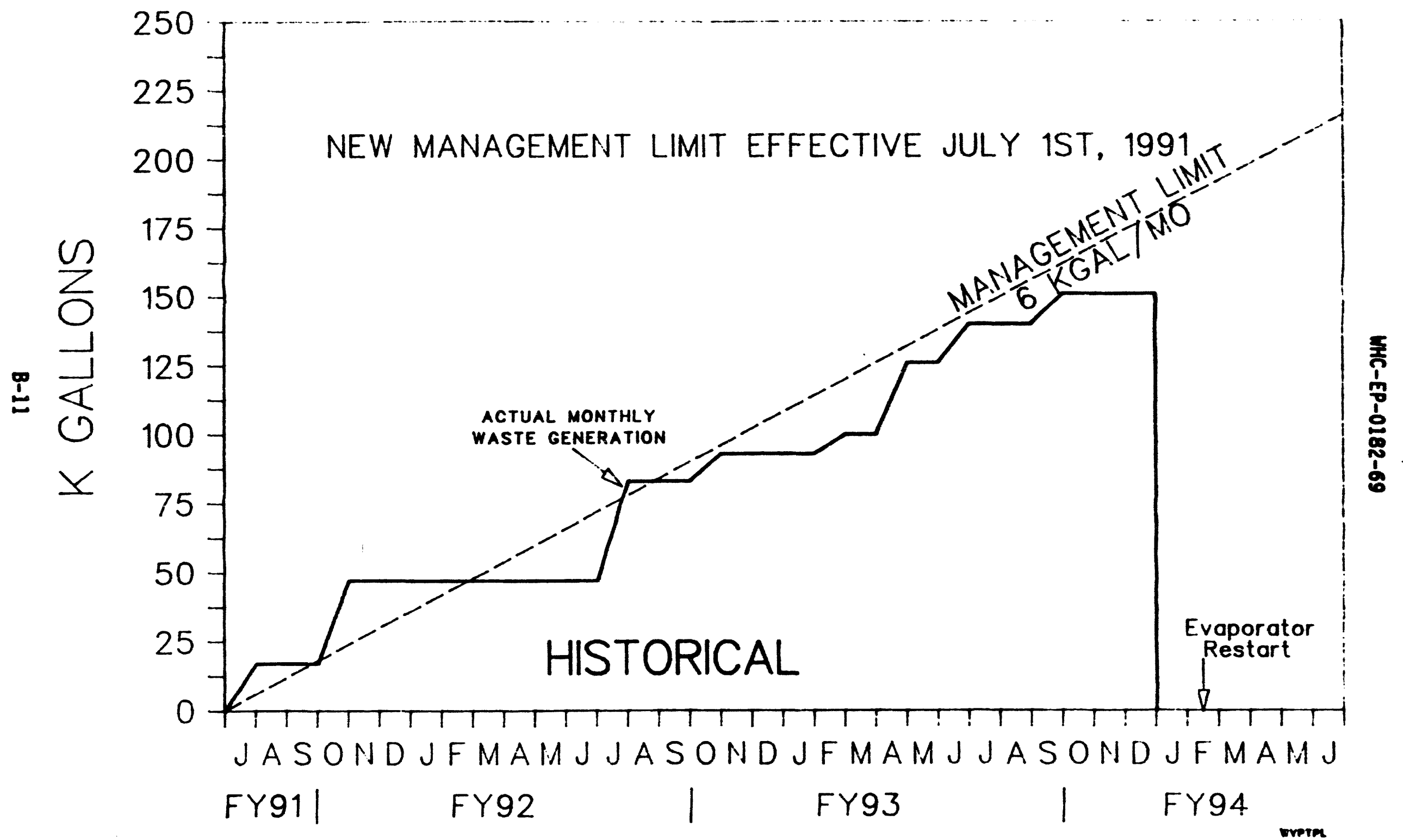

FIGURE B--8. T PLANT MONTHLY WASTE GENERATIONS 


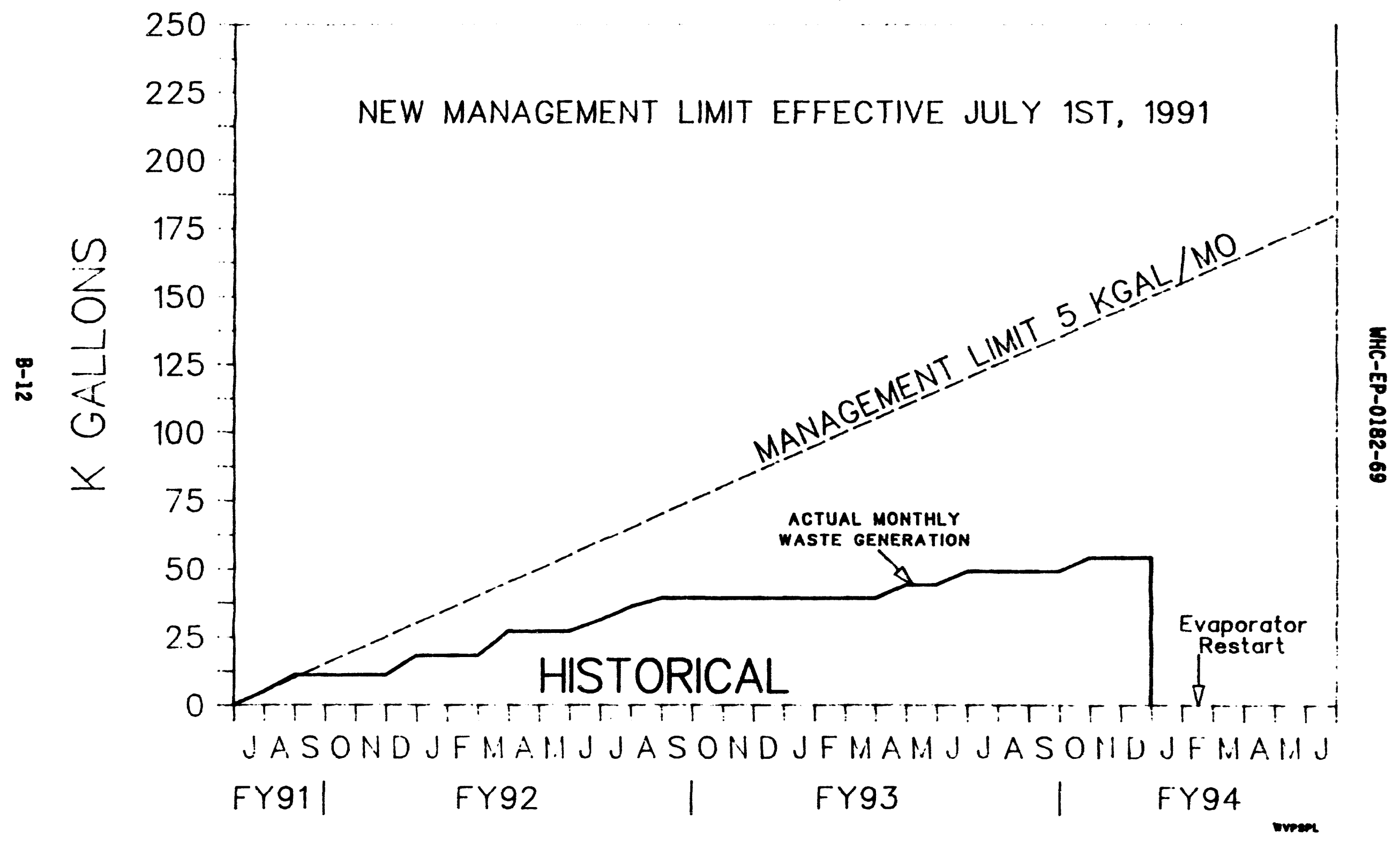

FIGURE B-9. S PLANT MONTHLY WASTE GENERATIONS 


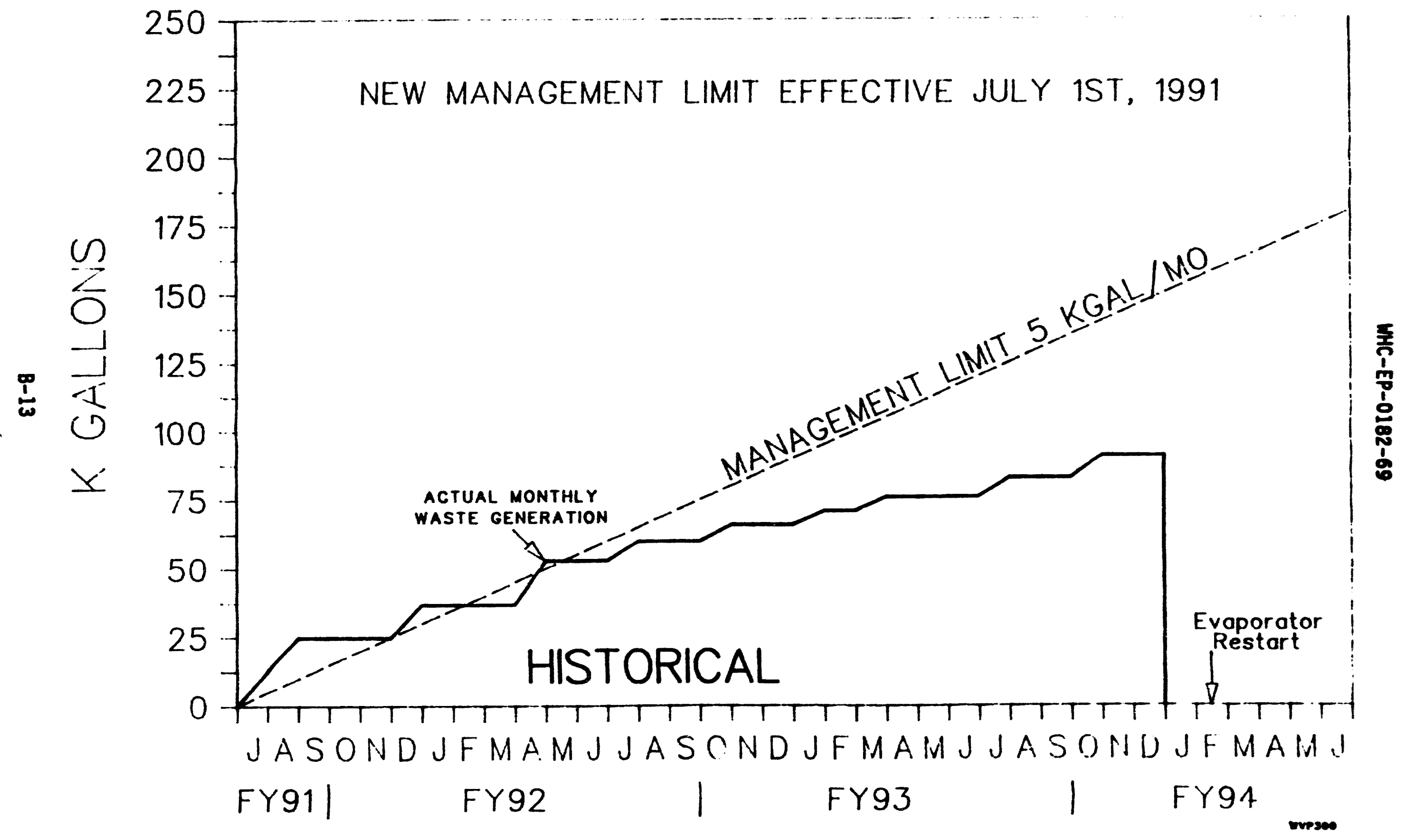

FIGURE B-10. 300 AREA MONTHLY WASTE GENERATIONS 
Table B-2. Double Shell Tank Waste Inventory for December 31, 1993 (page 1 of 2)

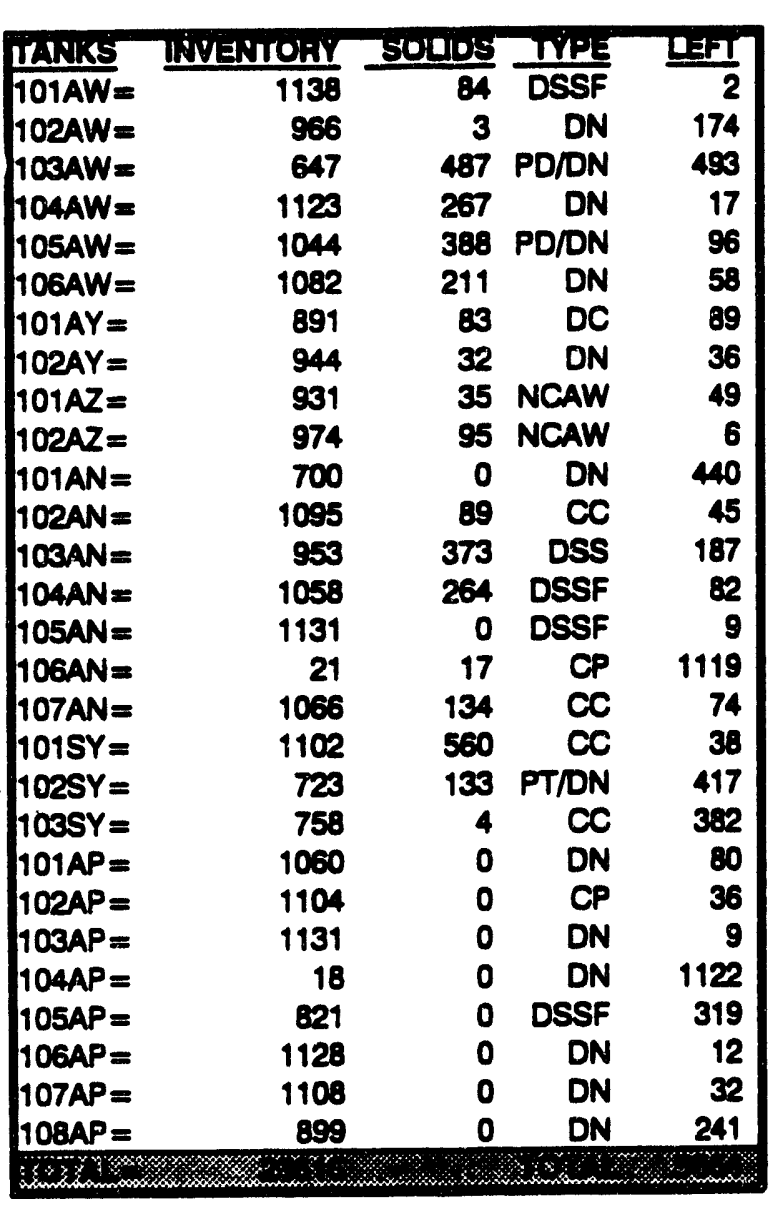

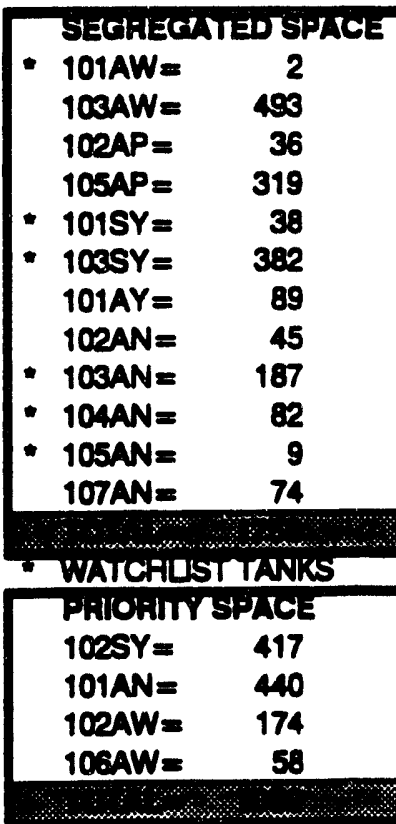

\begin{tabular}{|c|c|}
\hline \multicolumn{2}{|c|}{ 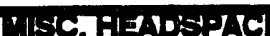 } \\
\hline $101 \mathrm{AP}=$ & 80 \\
\hline $103 A P=$ & 9 \\
\hline $106 A P=$ & 12 \\
\hline $107 A P=$ & 32 \\
\hline 104AW = & 17 \\
\hline 101AZ= & 49 \\
\hline $102 A Z=$ & 6 \\
\hline
\end{tabular}

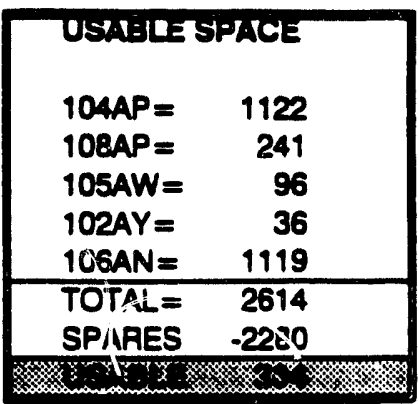

TOTALSPIACEAVAILABLE NON-AGING 27360 AGING = : 2 <

TOTALINVANiroliY 11/93 TOTAL 25525 $12 / 93$ TOTAL 25616

x

\begin{tabular}{|c|c|}
\hline UStat: & P $/ 40$ \\
\hline $\begin{array}{l}\text { 11/93 TOTAL } \\
12 / 93 \text { TOTAL }\end{array}$ & $\begin{array}{l}347 \\
334\end{array}$ \\
\hline
\end{tabular}

PHILARIT SHALE

$11 / 93$ TOTAL 1145

$12 / 93$ TOTAL 1089

Inventory Calculation by Waste Type:

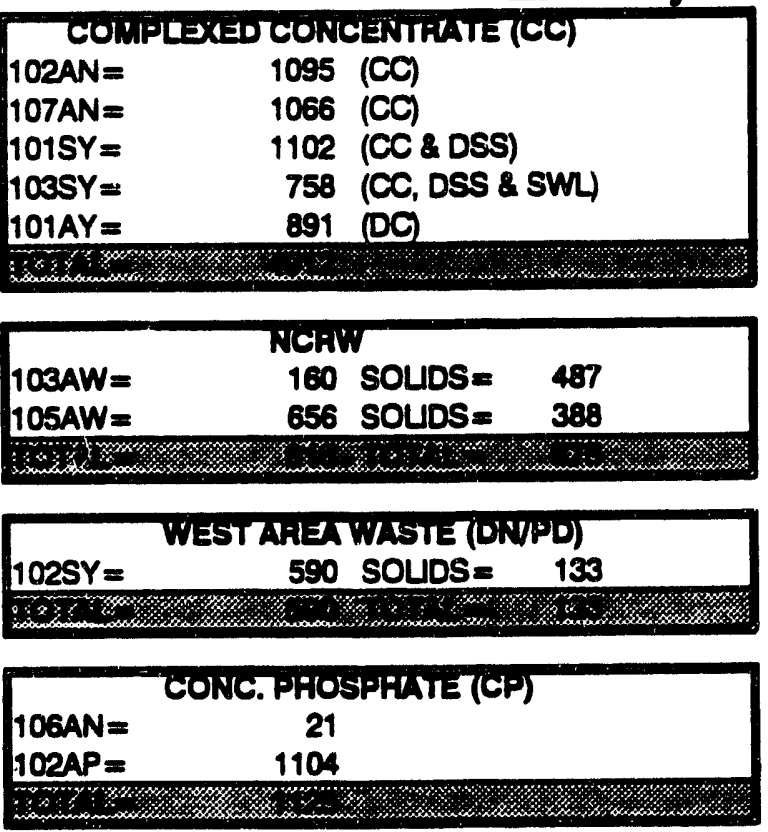
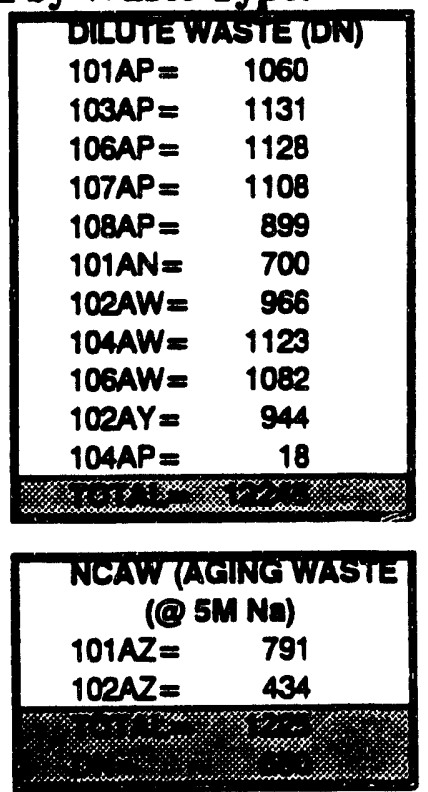

\begin{tabular}{|cr|}
\hline DSS/OSSF \\
105AP $=$ & 821 \\
$103 A N=$ & 953 \\
$104 A N=$ & 1058 \\
$105 A N=$ & 1131 \\
$101 A W=$ & 1138 \\
\hline
\end{tabular}

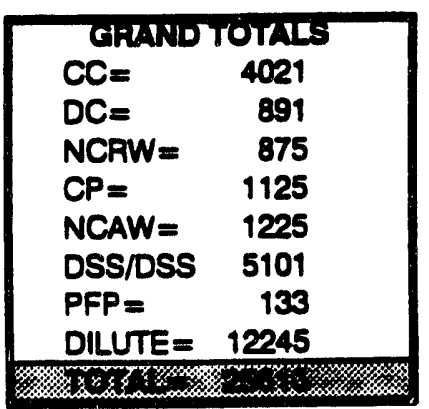

NOTE: All Values are in Kilogallons. 


$$
\text { WHC-EP-0182-69 }
$$

Table B-2. Double Shell Tank Waste Inventory for December 31, $1993 \quad$ (page 2 of 2)

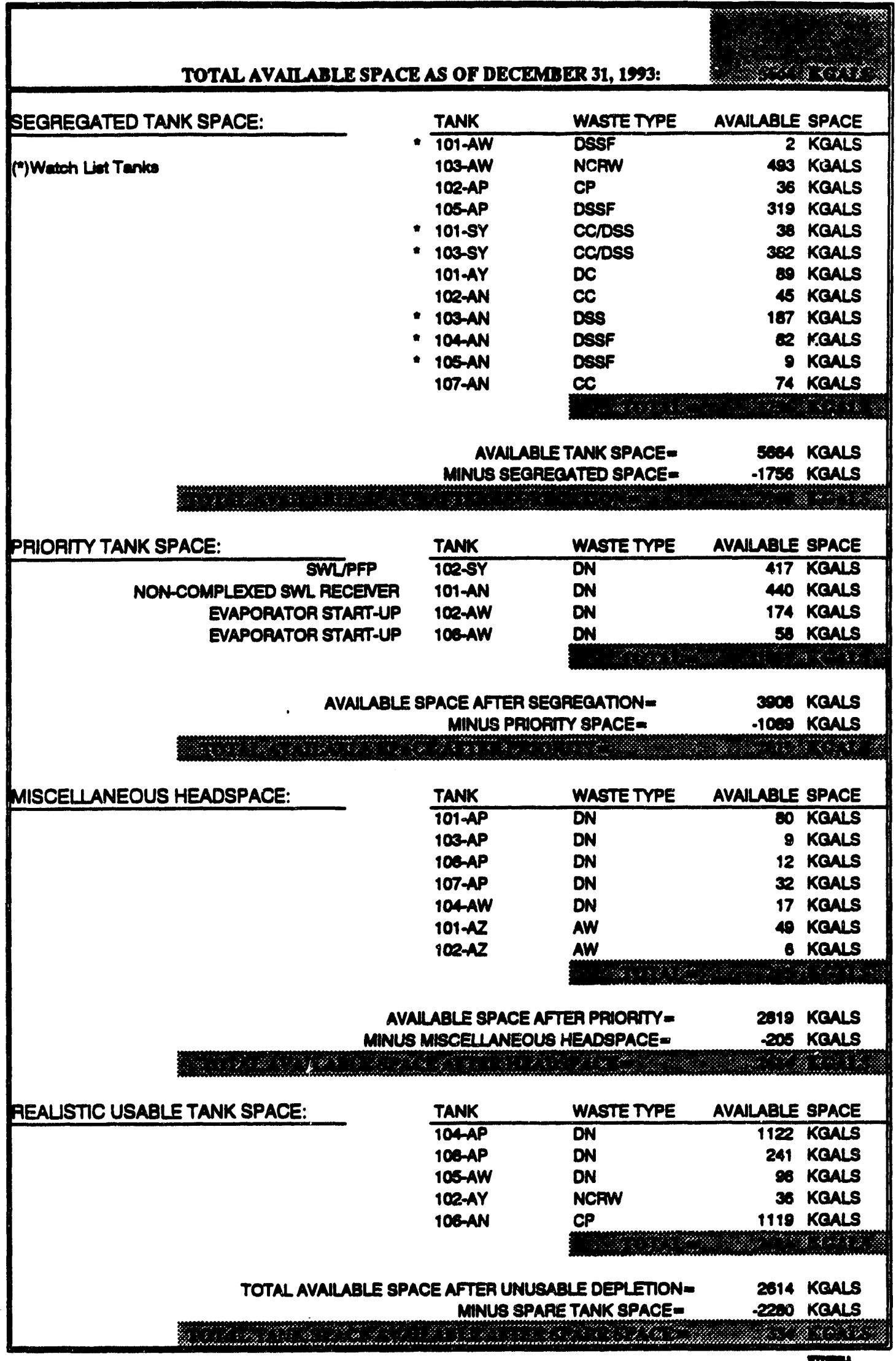


มHCC-EP-0182-69

This page intentionally left blank 
WHC-EP-0182-69

\section{APPENDIX C}

TANK AND EQUIPMENT CODE AND STATUS DEFINITIONS 
WHC-EP-0182-69

This page intentionally left blank 


\section{TANK AND EQUIPMENT CODE/STATUS DEFINITIONS \\ December 31, 1993}

\section{TANK STATUS CODES}

\section{MASTE TYPE}

$\begin{array}{ll}\text { AGING } & \text { Aging Waste (Neutralized Current Acid Waste [NCAW]) } \\ \text { CC } & \text { Complexant Concentrate Waste } \\ \text { CP } & \text { Concentrated Phosphate Waste } \\ \text { DC } & \text { Dilute Complexed Waste } \\ \text { DN } & \text { Dilute Non-Complexed Waste } \\ \text { DSS } & \text { Double-Shell Slurry } \\ \text { DSSF } & \text { Double-Shell Slurry Feed } \\ \text { NCPLX } & \text { Non-Complexed Waste } \\ \text { PD/PN } & \text { Plutonium-Uranium Extraction (PUREX) Neutralized Cladding } \\ \text { PT } & \text { Removal Waste (NCRW) transuranic waste (TRU) } \\ \text { Plutonium Finishing Plant (PFP) TRU Solids } & \\ \text { TANK USE (DOUBLE-SHELL TAMKS ONLY) }\end{array}$

$\begin{array}{ll}\text { CWHT } & \text { Concentrated Waste Holding Tank } \\ \text { DRCVR } & \text { Dilute Receiver Tank } \\ \text { EVFD } & \text { Evaporate Feed Tank } \\ \text { GRTFD } & \text { Grout Feed Tank } \\ \text { SRCVR } & \text { Slurry Receiver Tank }\end{array}$

\section{SOLID AND LIOUID VOLUME DETERMIMATION METHODS}

F Food Instrument Company (FIC) Automatic Surface Level Gauge $M \quad$ Manual Tape Surface Level Gauge

P Photo Evaluation

S Sludge Level Measurement Device

\section{DEFINITIONS}

\section{WASTE TANKS - GENERAL}

Waste Tank Safety Issue

Watch List Tank
A potentially unsafe condition in the handling of waste material in underground storage tanks that requires corrective action to reduce or eliminate the unsafe condition.

An underground storage tank containing waste that requires special safety precautions because it may have a serious potential for release of high level radioactive waste because of uncontrolled increases in temperature or pressure. Special restrictions have been placed on these tanks by "Safety Measures for Waste Tanks at Hanford Nuclear Reservation," Section 3137 of the National Defense Authorization Act for Fiscal Year 1991, November 5, 1990, Public Law 101-510, (also known as the Wyden Amendment). 


\section{MASTE TYPES}

Aging Waste

(AGING)

Concentrated Complexant (CC)

Concentrated Phosphate Waste (CP)

Dilute Complexed Waste (DC)
High level, first cycle solvent extraction waste from the PUREX plant (NCAW)

Concentrated product from the evaporation of dilute complexed waste.

Waste originating from the decontamination of the $\mathrm{N}$ Reactor in the $100 \mathrm{~N}$ Area. Concentration of this waste produces concentrated phosphate waste.

Characterized by a high content of organic carbon including organic complexants:

ethylenediaminetetra-acetic acid (EDTA), citric acid, and hydroxyethyl-ethylenediaminetriacetic acid (HEDTA), being the major complexants used. Main sources of DC waste in the DST system are saltwell liquid inventory (from SSTs).

Dilute Non-Complexed Waste (DN)

Low activity liquid waste originating from $T$ and S Plants, the 300 and 400 Areas, PUREX facility (decladding supernatant and miscellaneous wastes), $100 \mathrm{~N}$ Area (sulfate waste), B Plant, saltwells, and PFP (supernate).

Double-Shell

STurry (DSS)

Double-Shell slurry Feed (DSSF)

Non-complexed (NCPLX)

PUREX Decladding (PD/PN)

PFP TRU Solids (PT)

Drainable Interstitial Liquid (DIL)
Waste that exceeds the sodium aluminate saturation boundary in the evaporator without exceeding receiver tank composition limits. For reporting purposes, DSS is considered a solid.

Waste concentrated just before reaching the sodium aluminate saturation boundary (of $6.5 \mathrm{~mol}$ ar hydroxide) in the evaporator without exceeding receiver tank composition limits. This form is not as concentrated as DSS.

General waste term applied to all Hanford Site liquors not identified as complexed.

PUREX Neutralized Cladding Removal Waste (NCRW) is the salids portion of the PUREX plant neutralized cladding removal waste stream; received in Tank Farms as a slurry. NCRW solids are classified as transuranic (TRU) waste.

TRU solids fraction from PFP PIant operations.

Interstitial liquid that is not held in place by capillary forces, and will therefore migrate or move by gravity. 
Supernate

Ferrocyanide

MASTE STATUS

In-Service Tank

Out-of-Service

Tank
The liquid above the solids in waste storage tanks.

A compound of iron and cyanide commonly expressed as $\mathrm{FeCN}$. The actual formula for the ferrocyanide anion is $\left[\mathrm{Fe}(\mathrm{CN})_{6}\right]^{-4}$.

The waste classification of a tank being used, or planned for use, for the storage of liquid (in excess of a minimum supernatant liquid heel) in conjunction with production and/or waste processing.

A tank which does not meet the definition of an inservice tank. Before September 1988, these tanks were defined as inactive in this report. [Note: All single-shell tanks (SST) are out of service.]

\section{STABILIZATION (Single-Shell Tanks only)}

Interim

Stabilized

(IS)
A tank which contains less than $50,000 \mathrm{gal}$ of drainable interstitial liquid and less than 5,000 gal of supernatant liquid. If the tank was jet pumped to achieve interim stabilization, then the jet pump flow must also have been at or below $0.05 \mathrm{gpm}$ before interim stabilization criteria is met.

\section{INTRUSION PREVENTION (ISOLATION) Single-Shell Tanks only}

Partially

Interim Isolated

(PI)

Interim 'solated (II)

Intrusion Prevention (IP)
The administrative designation reflecting the completion of the physical effort required for Interim Isolation except for isolation of risers and piping that is required for jet pumping or for other methods of stabilization.

The administrative designation reflecting the completion of the physical effort required to minimize the addition of liquids into an inactive storage tank, process vault, sump, catch tank, or diversion box. In June 1993, Interim Isolation was replaced by Intrusion Prevention.

Intrusion Prevention is the administrative designation reflecting the completion of the physical effort required to minimize the addition of liquids into an inactive storage tank, process vault, sump, catch tank, or diversion box. Under no circumstances are electrical or instrumentation devices disconnected or disabled during the intrusion prevention process (with the exception of the electrical pump), in accordance with WHC-SD-WMSAR-006 REV 2, Single-Shell Tank I solation Safety Analysis Report, March 1986. 
IAK INTEERITY

Sound

Assumed Leaker

The integrity classification of a waste storage tank for which survellilance data indicate no loss of ilquid attributed to a breach of integrity.

The integrity classification of a waste storage tank for which survelliance data indicate a loss of liquid attributed to a breach of integrity.

Assumed Re-Leaker A condition that exists after a tank has been declared as an "assumed leaker" and then the survelliance data indicates a new loss of liquid attributed to a breach of integrity.

TAM IMESTIEATION

Intrusion

A term used to describe the infiltration of liquid into a waste tank.

\section{SURYEILLNCE IMSTRINEITATION}

Drywells

Drywells are vertical boreholes with 6-in. (internal diameter) carbon steel casings positioned radially around SSTs. Periodic monitoring is done by gamma radiation or neutron sensors to obtain scan profiles of radiation or moisture in the soil as a function of well depth, which could be indicative of tank leakage. These wells range between 50 and $250 \mathrm{ft}$ in depth, and are monitored between the range of 50 to $150 \mathrm{ft}$. The wells are sealed when not in use. They are called drywells because they do not penetrate to the water table and are therefore usualiy "dry." There are 759 drywells which are monitored on various frequencies.

Laterals

Laterals are horizontal drywells positioned under single-shell waste storage tanks to detect radionuclides in the soil which could be indicative of tank leakage. These drywells are monitored by radiation detection probes. Laterals are 4-in. inside diameter steel pipes located 8 to $10 \mathrm{ft}$ below the tank's concrete base. There are three laterals per tank. Laterals are located only in $A$ and $S X$ farms.

Surface Levels The surface level measurements in all waste storage tanks are monitored by manual or au+omatic conductivity probes, and recorded and transmitted or inputted to the Computer Automated Surveillance System (CASS). 
Automatic FIC

Annulus

Liquid Observation Well (LOW)

Thermocouple (TC)
An automatic waste surface level measurement device is manufactured by the Food Instrument Company (FIC). The instrument consists of a conductivity electrode (plummet) connected to a calibrated steel tape, a steel tape reel housing and a controller that automatically raises and lowers the plummet to obtain a waste surface level reading. The controller can provide a digital display of the data and also transmit the reading to the CASS. Some tanks have gauges connected to CASS and others are read manualiy.

The annulus is the space between the inner and outer shells on DSTs. Drain channels in the insulating and/or supporting concrete carry any leakage to the annulus space where conductivity probes are installed. Alarms from the annunciators are received by CASS. Continuous Air Monitoring (CAM) alarms are also located in the annulus. The annulus conductivity probes and radiation detectors are the primary means of leak detection for all DSTs.

In-tank liquid observation wells are used for monitoring the interstitial liquid level (ILL) in single-shell waste storage tanks. The wells are constructed of fiberglass or TEFZEL *-reinforced epoxy-polyester resin, sized to extend to within 1 in. of the botton of the tank steel 1iner. They are sealed at their bottom ends and have 2 nominal outside diameter of $3.5 \mathrm{in}$. Two probes are used to monitor changes in the ILL: gamma and neutron, which can indicate intrusions or leakage by increases or decreases in the ILL. There are 58 LOWs (56 are in operation) installed in SSTs that contain or are capable of containing greater than 50,000 gal of drainable interstitial liquid, and in two DSTs only. The LOWs installed in two DSTs, (102-SY and 103-AW Tanks), are constructed of steel and are used for spectal survetllance purposes only.

A thermocouple is a thermoelectric device used to measure temperature. More than one thermocouple on a device (probe) is called a thermocouple tree. In DSTs there may be one or more thermocouple trees in risers in the primary tank. In addition, in DSTs only, there are thermocouple elements installed in the insulating concrete, the lower primary tank knuckle, the secondary tank concrete foundation, and in the outer structural concrete. These monitor temperature gradients within the concrete walls,

*TEFZEL, a trademark of E. I. du Pont de Nemours \& Company 
bottom of the tank, and the domes. In SSTs, there may be one or more thermocouple trees instailed directly in a tank, although some SSTs do not have any trees installed. A single thermocouple may be installed in a riser, or lowered down an existing riser or LOW. There are also four thermocouple laterals beneath Tank 105-A in which temperature readings are taken in 34 thermocouples.

In-tank Photography
In-tank photographs may be taken to aild in resolving in-tank measurement anomalies and determine tank integrity. Photographs help determine sludge and liquid levels by visual examination.

\section{IMETTORY ACO STATUS BY TAKK - COLUM GALCULATHOMS (STMGLE-SHEHL TANKS)}

\section{COLUIN HE.DTIN}

Total Waste

Solids Volume plus Supernatant liquid.

Supernatant Liquid

Drainable Liquid Remaining minus Drainable Interstitial. Supernate is usually derived by subtracting the solids level measurement from the liquid level measurement.

Drainable Interstitial

Pumped This Month

Total Pumped

Drainable Liquid

Remaining

Pumpable Liquid Remaining

Sludge

Saltcake
Drainable Liquid Remaining minus Supernate.

Drainable Interstitial Liquid is calculated based on the saltcake and sludge volumes, using average porosity values or actual data for each tank, when available.

Totai pumped this month

Cumulative total pumped 1979 to date.

Supernate plus Drainable Interstitial.

Drainable Liquid Remaining less undrainable heel volume.

Solids formed during sodium hydroxide additions to waste. sludge usually was in the form of suspended solids when the waste was originally received in the tank from the waste generator. In-tank photographs may be used to estimate the volume.

Results from crystalization and precipitation after concentration of liquid waste, usually in an evaporator. If saltcake is layered over siudge, it is only possible to measure total solids volume. In-tank photographs may be used to estimate the saltcake volume. 


\section{MHC-EP-0182-69}

Solids Volume Indicates the latest update of any change in the solids Update volume.

Solids Update Indicates the source or basis of the latest solids Source - See volume update.

Footnote

Last Photo Date of latest in-tank photographs taken.

Date

Change Since Indicates any change made since the previous month. Last Monthly Explanation for the change follows the Inventory and Report Status by Tank section. 


\section{MHC-EP-0182-69}

This page intentionally left blank 
HHC -EP-0182-69

\section{APPENDIX D}

\section{TANK FARM CONFIGURATION, STATUS, AND} FACILITY CHARTS 
WHC-EP-0182-69

This page intentionally left blank.

$D-2$ 


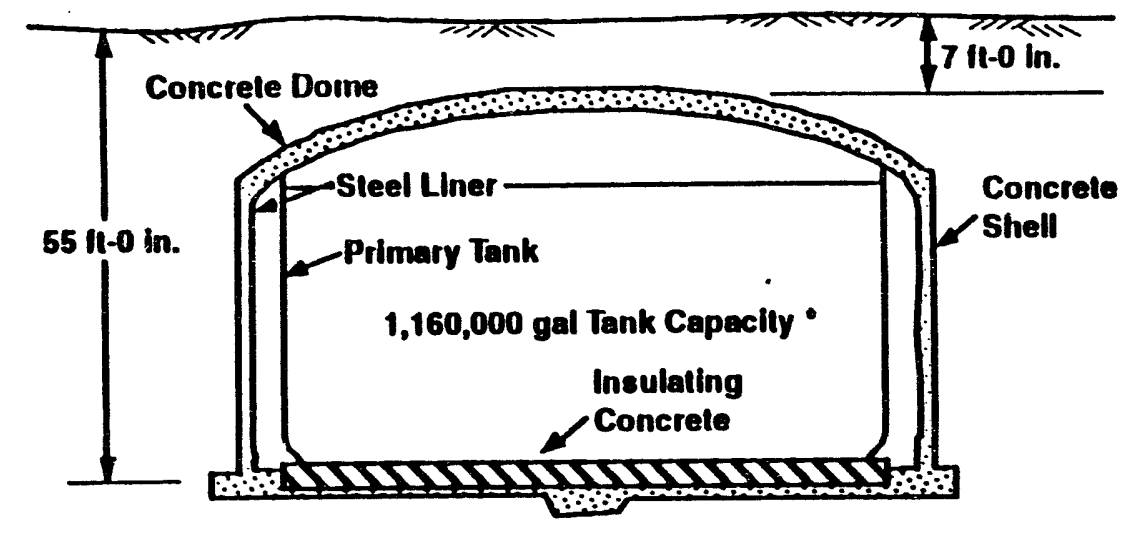

75 in Diameler Double-Shell Tank Tank Farms: AN, AP, AW, AY, AZ, SY

号

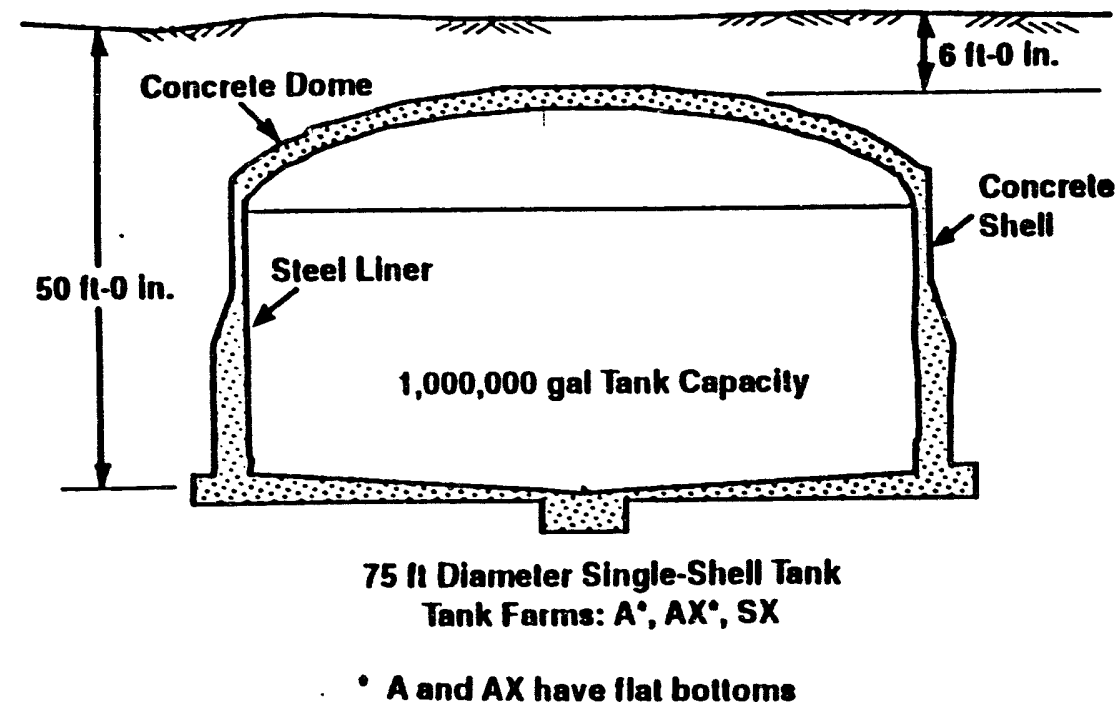

- A and $A X$ have flat bolloms

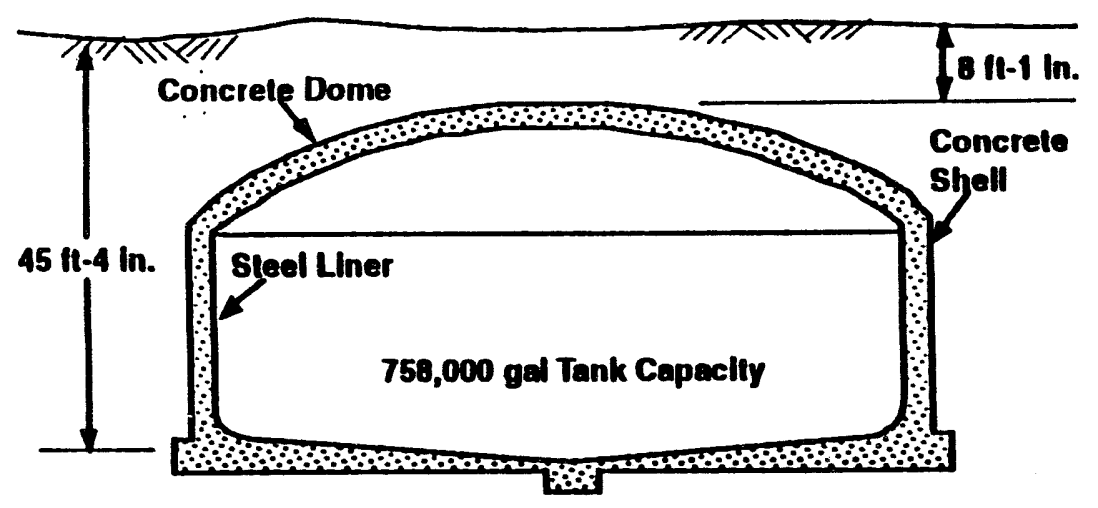

75 it Diameter Singlo-Shell Tank Tank Farms: BY, S, TX, TY

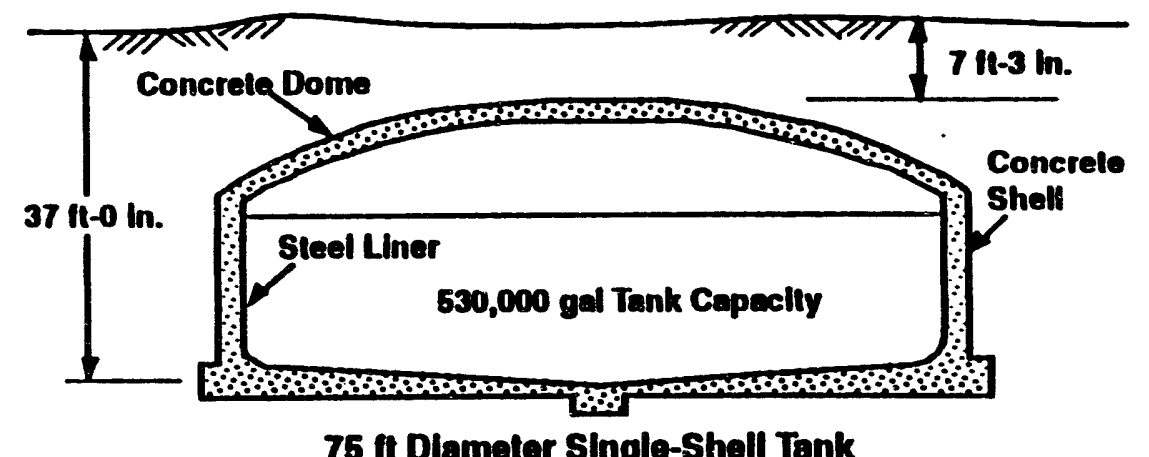
Tank Farms: B, BX, C, T, U

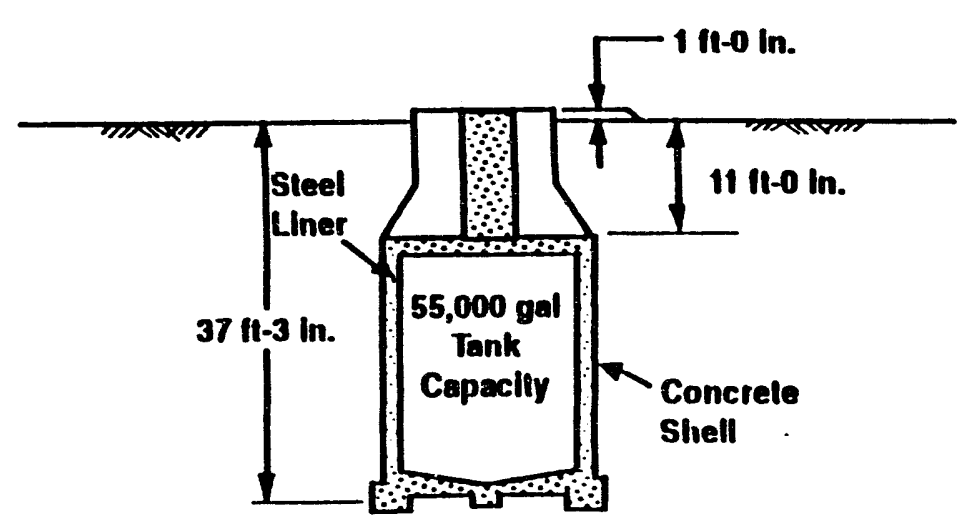

20 it Diameler Single-Shell Tank Tank Farms: B, C, T, U

Figure D-1. High-Level Waste Tank Configuration 


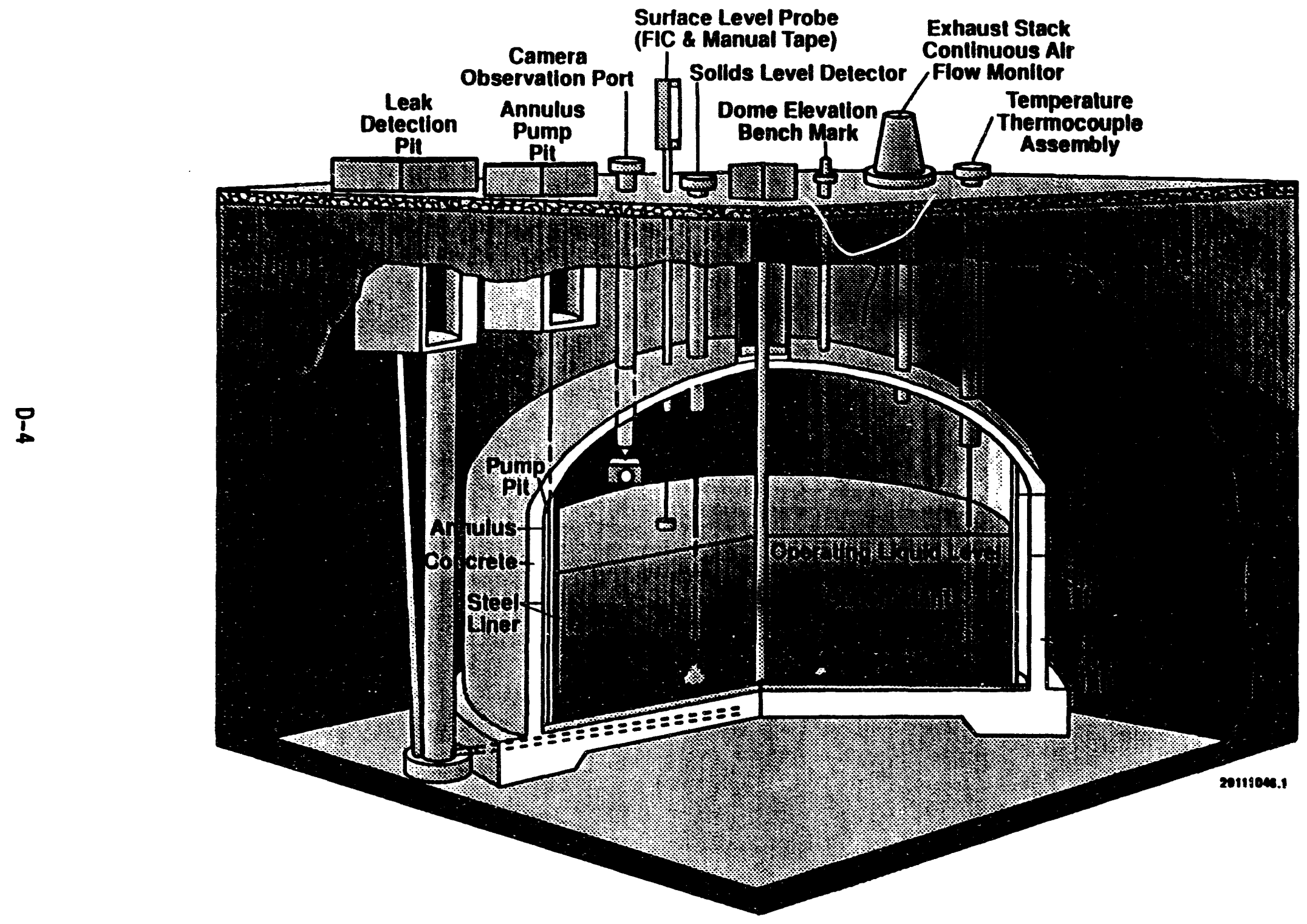

Figure D-2. Double-Shell Tank Instrumentation Configuration 


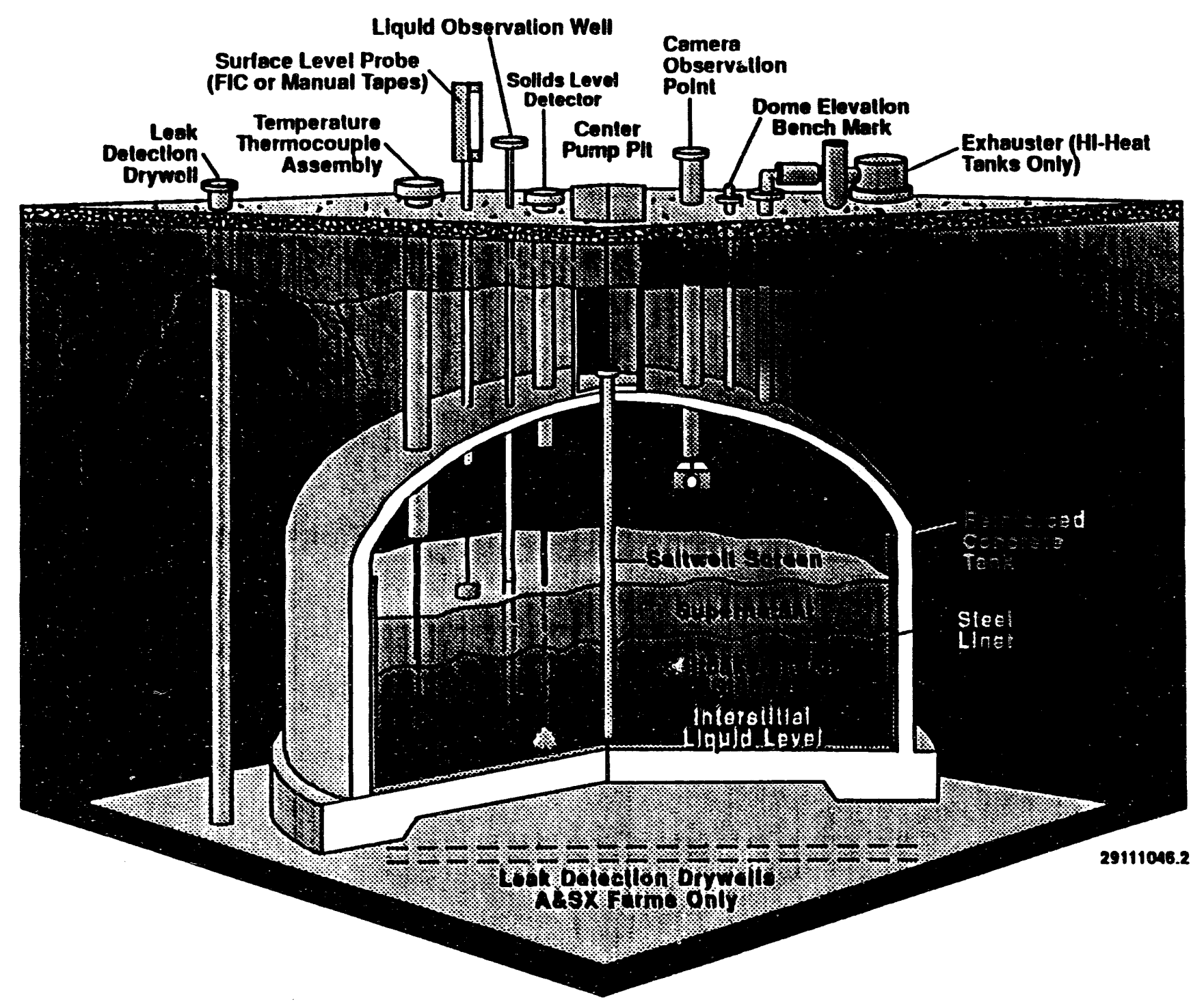

Figure D-3. Single-Shell Tank Instrumentation Configuration 
WHC-EP-0182-69

This page intentionally left blank

D-6 


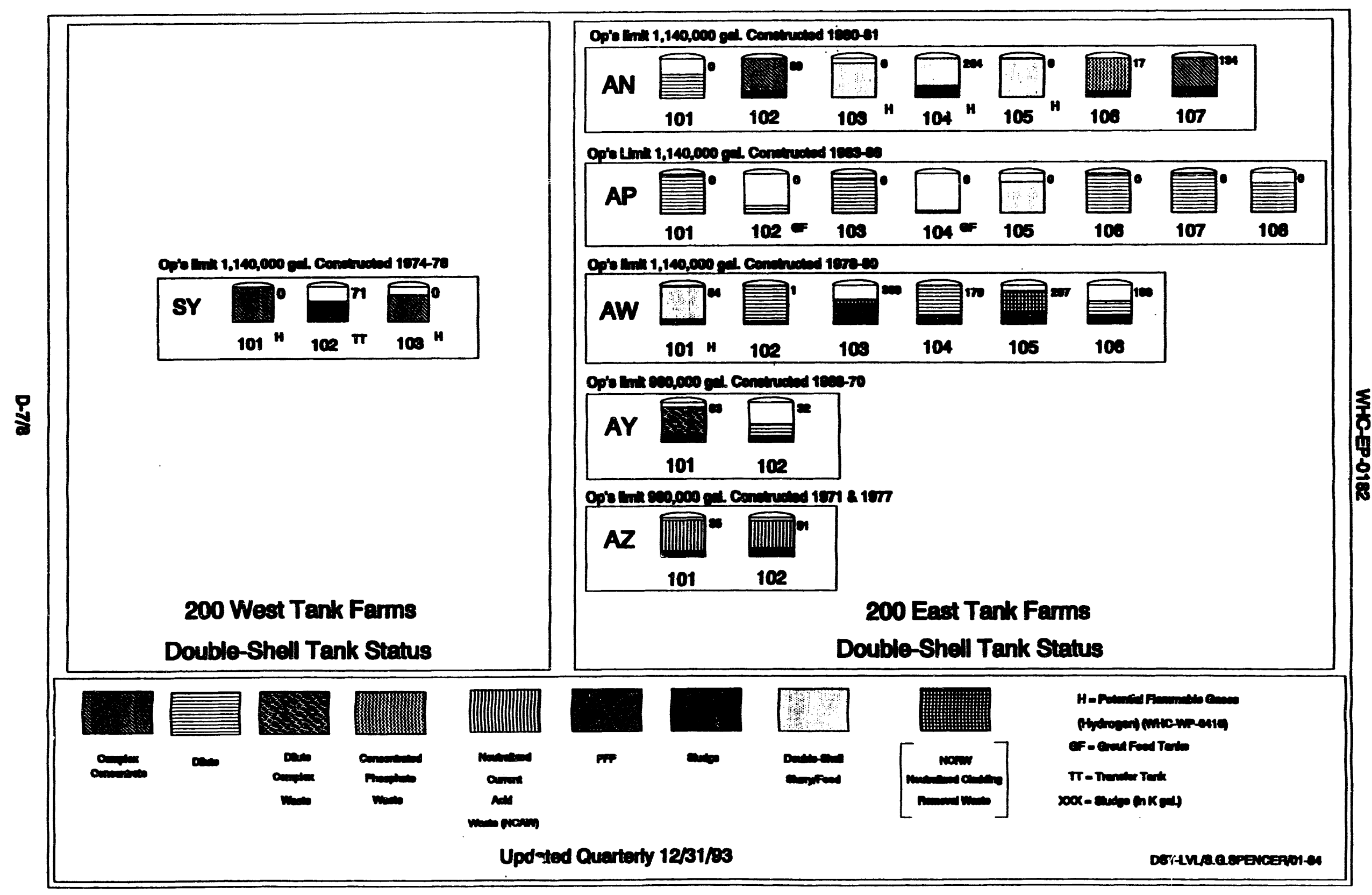

Figure D-4. Doublo-Shall Tenk Statue 
WHC-EP-0182

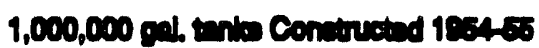

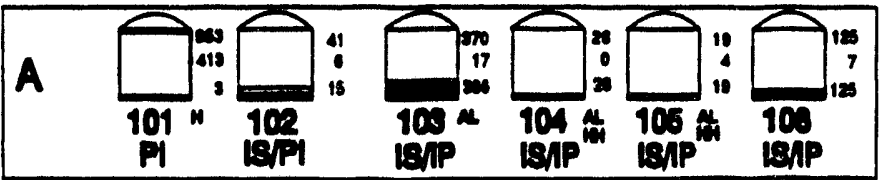

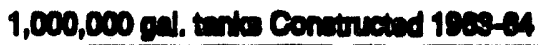

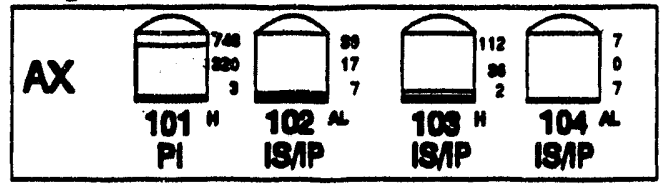

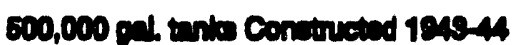

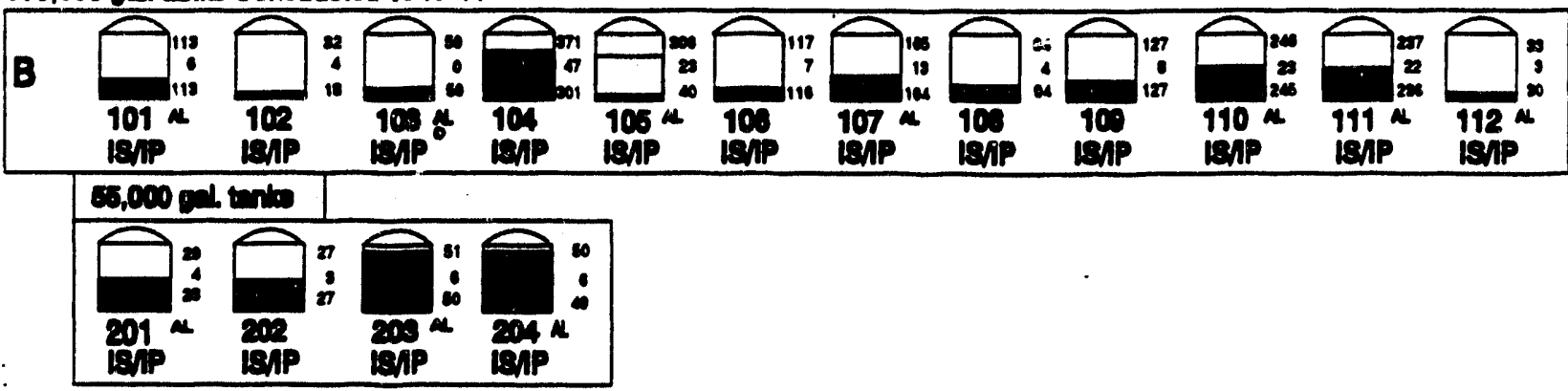

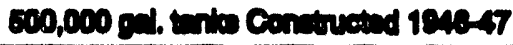

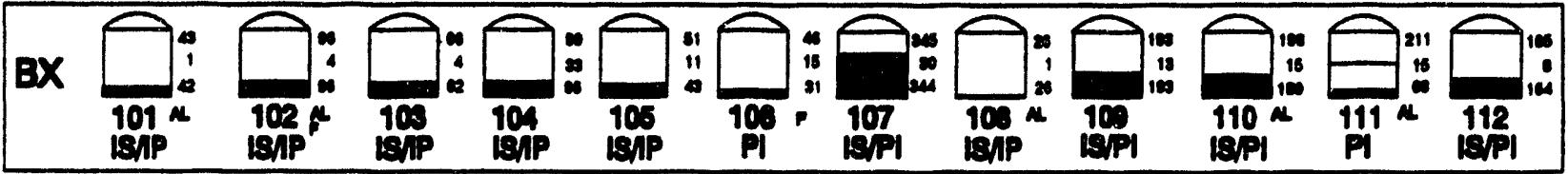

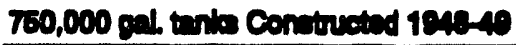

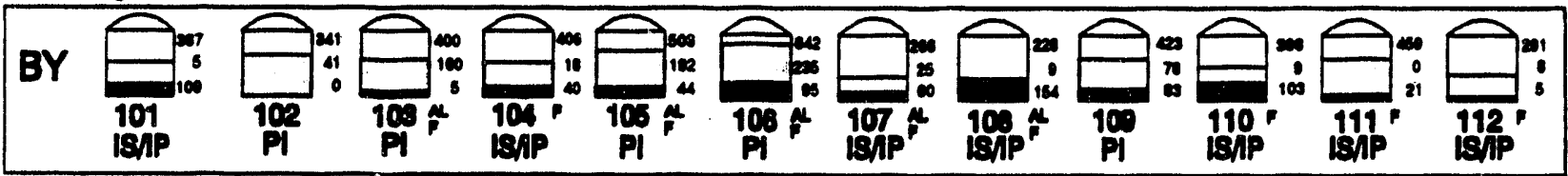

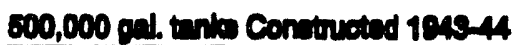

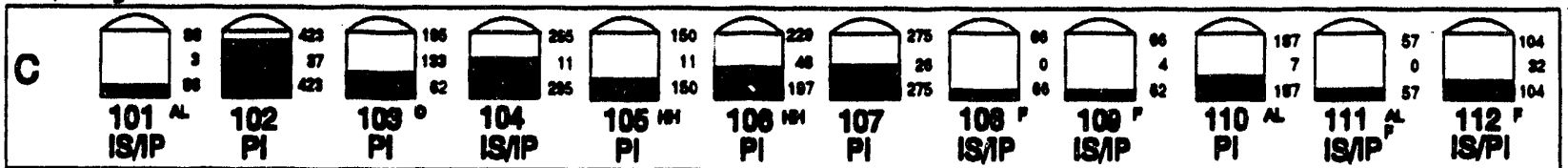

E5,000 gal tonta

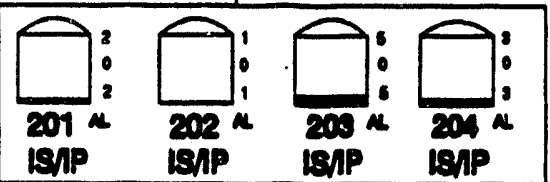

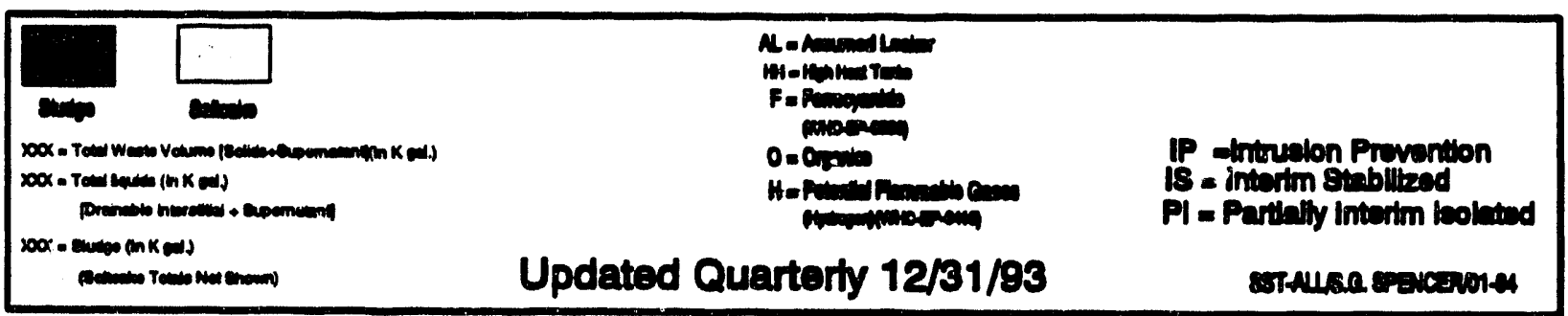

Figure D-5. 200E Single-Shell Tank Status 


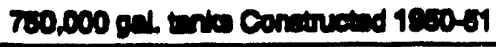

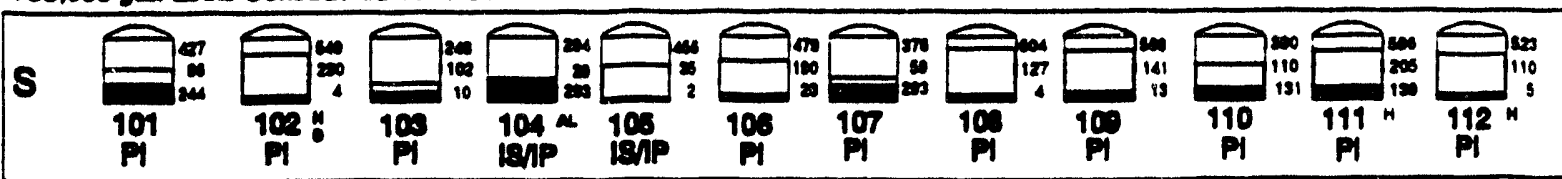

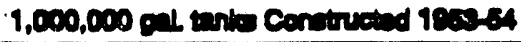

SX $\sum_{101}^{101}$

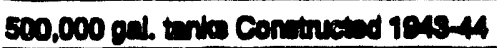

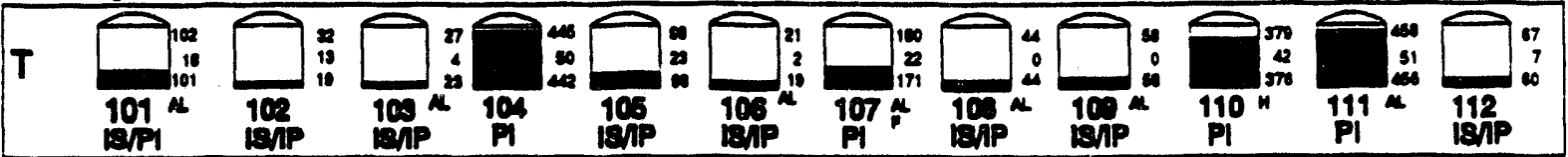

86,000 gel. tentos

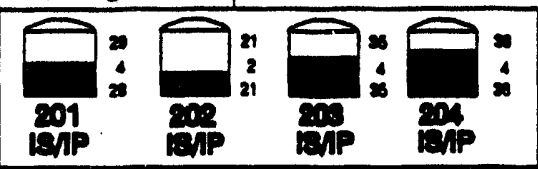

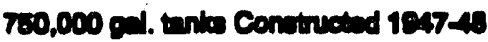

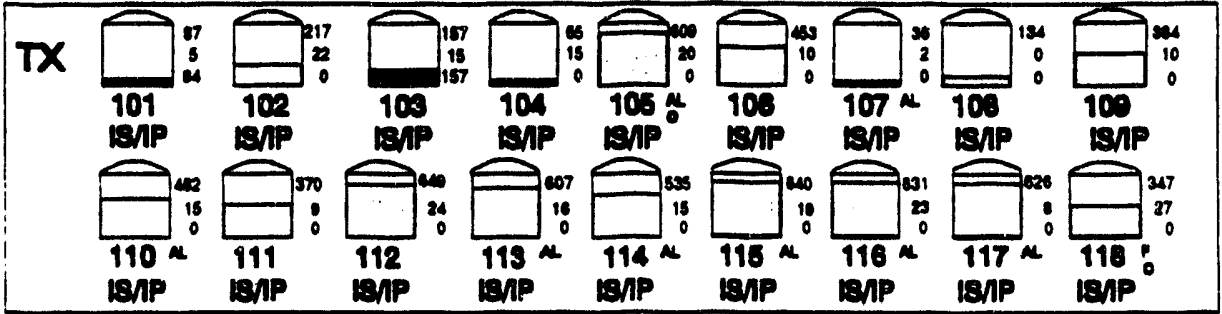

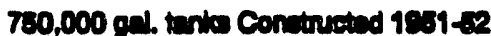

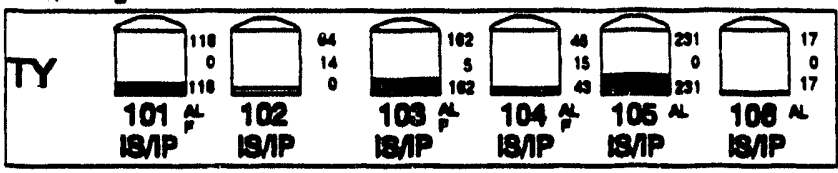

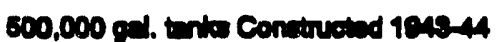

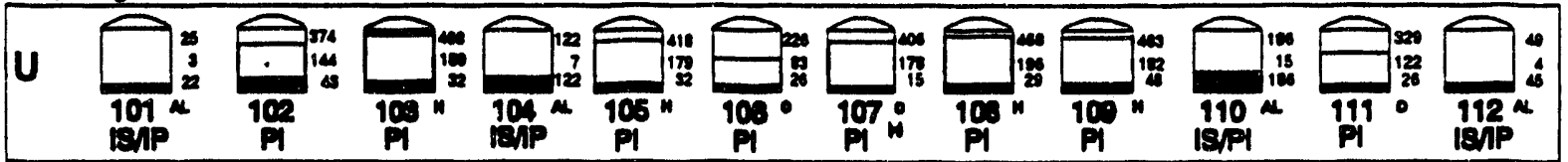

65,000 gel ando

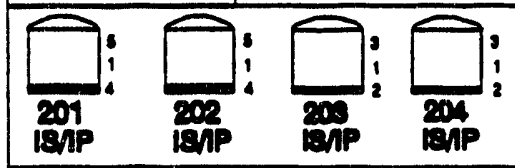

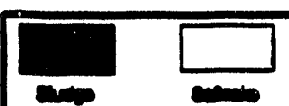

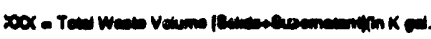

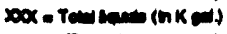

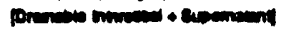

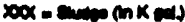

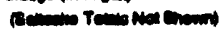

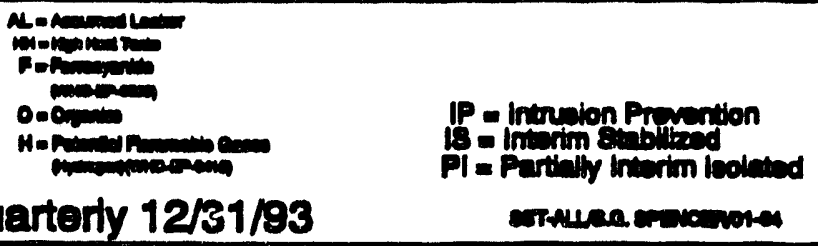

Updated Quarterly 12/31/93
IP - Intruion Prumention

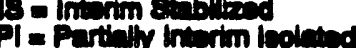

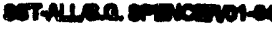

Fig. D-6. 200W Single-Shell Tank Status 


\section{East}

Hanford Tank Farm Facilities

Note: All single-shell tanks were removed from service (not allowed to receive waste) on or before November 21, 1980

Single Walled Pipe Direct Buried

All Tanks 75 Foot Diameter

(Except 55.000 gal 20 foot dia.)
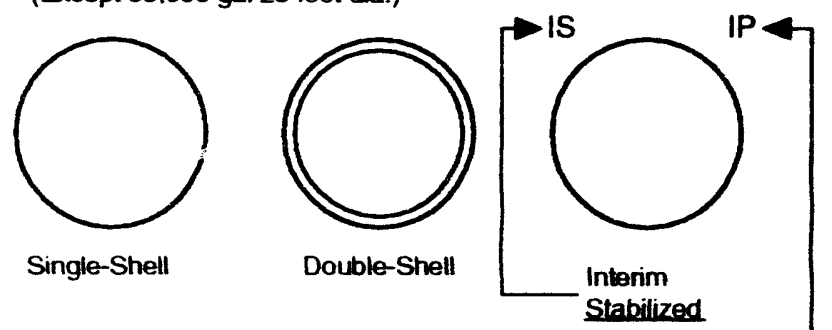

$$
\begin{gathered}
\text { Sound } \\
\text { DCPT }=\begin{array}{c}
\text { Double Contained } \\
\text { Receiver Tank }
\end{array} \\
\text { NHW }=\begin{array}{c}
\text { Neutralized } \\
\text { High Level Waste }
\end{array} \\
\text { PAW }=\text { PUREX Acid Waste }
\end{gathered}
$$
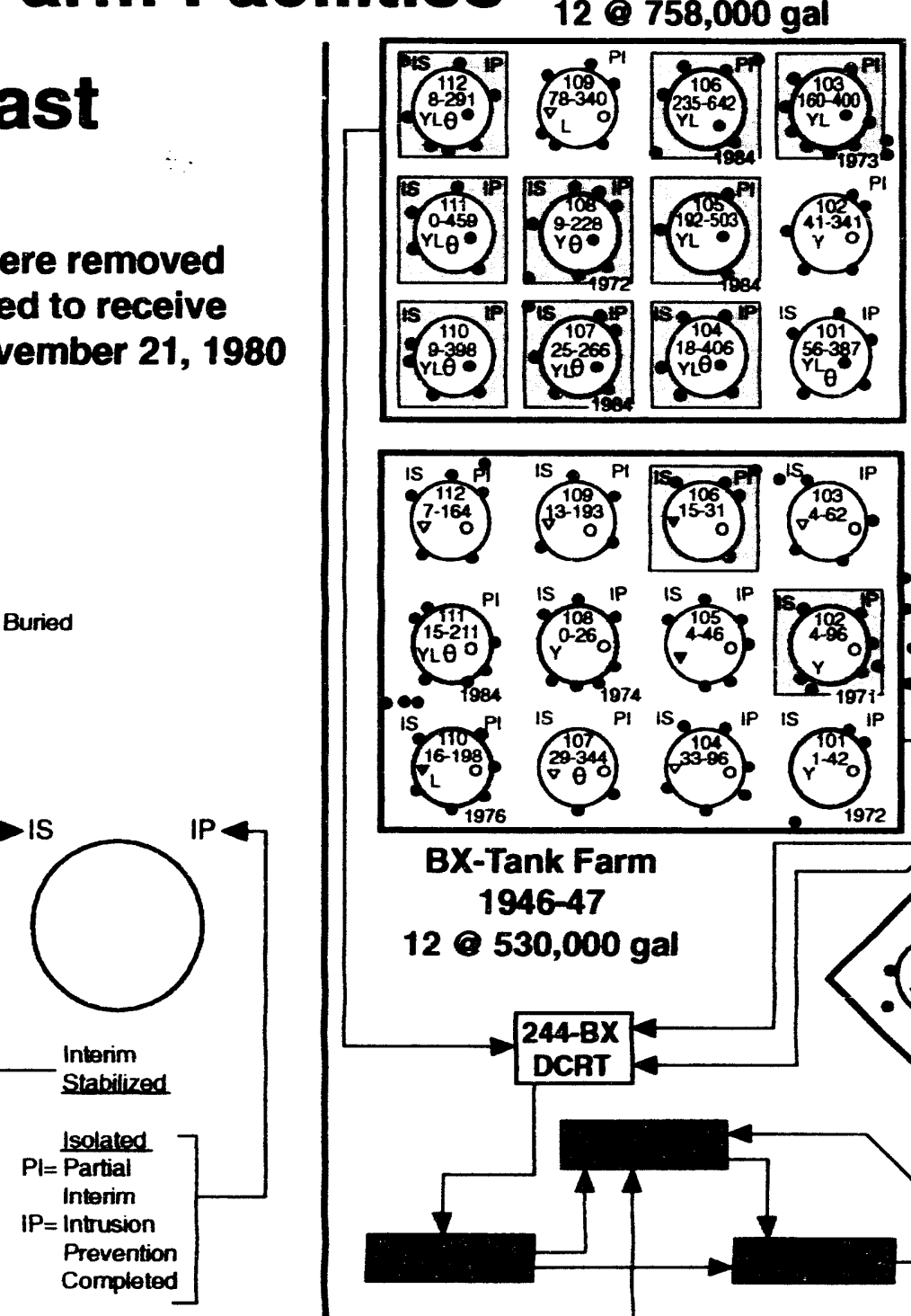

1943-44

$12 \odot 530,000 \mathrm{gal}$

4 ?5,000 gal
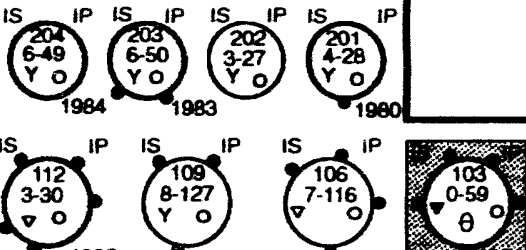

is 15

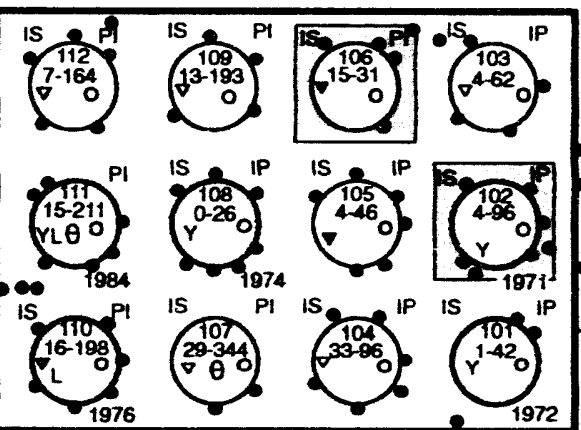
IP is

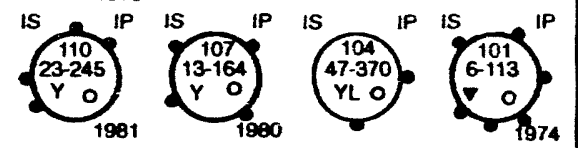

BX-Tank Farm

1946-47

$12 \div 530,000 \mathrm{gal}$

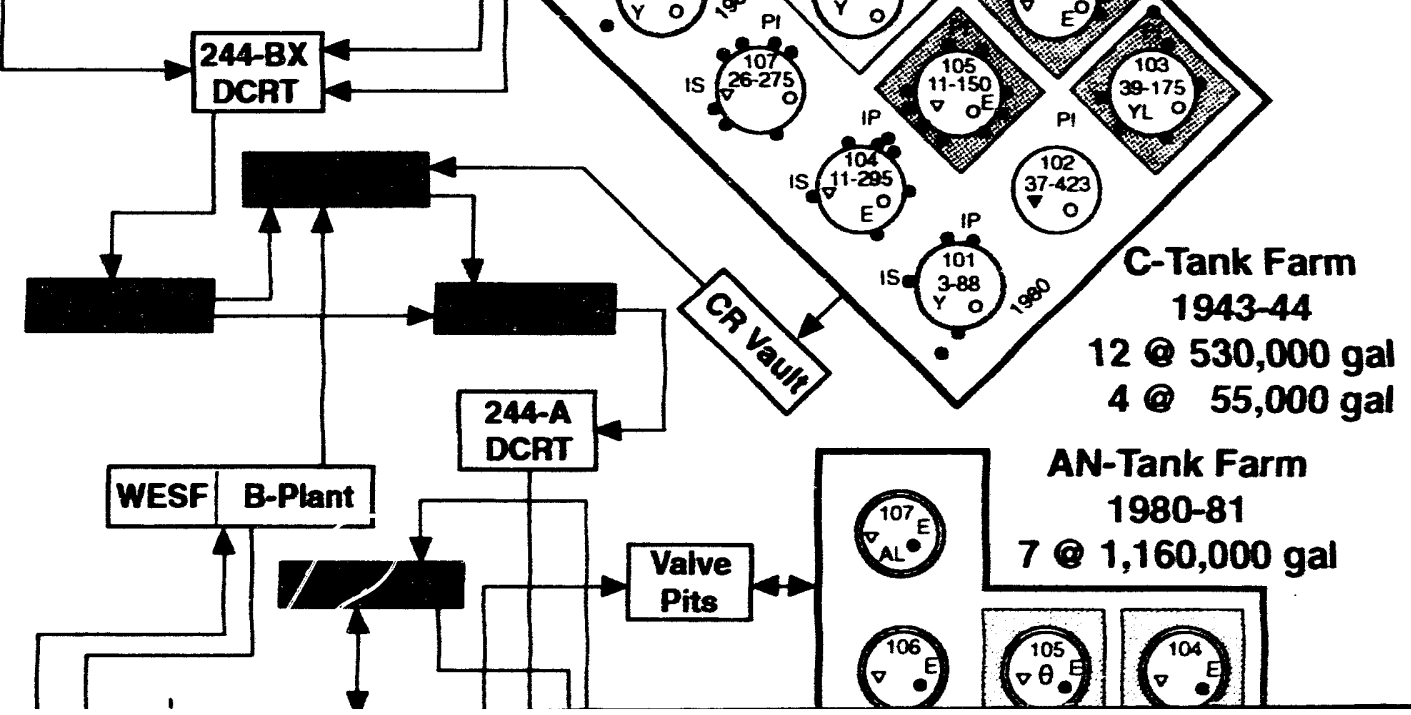




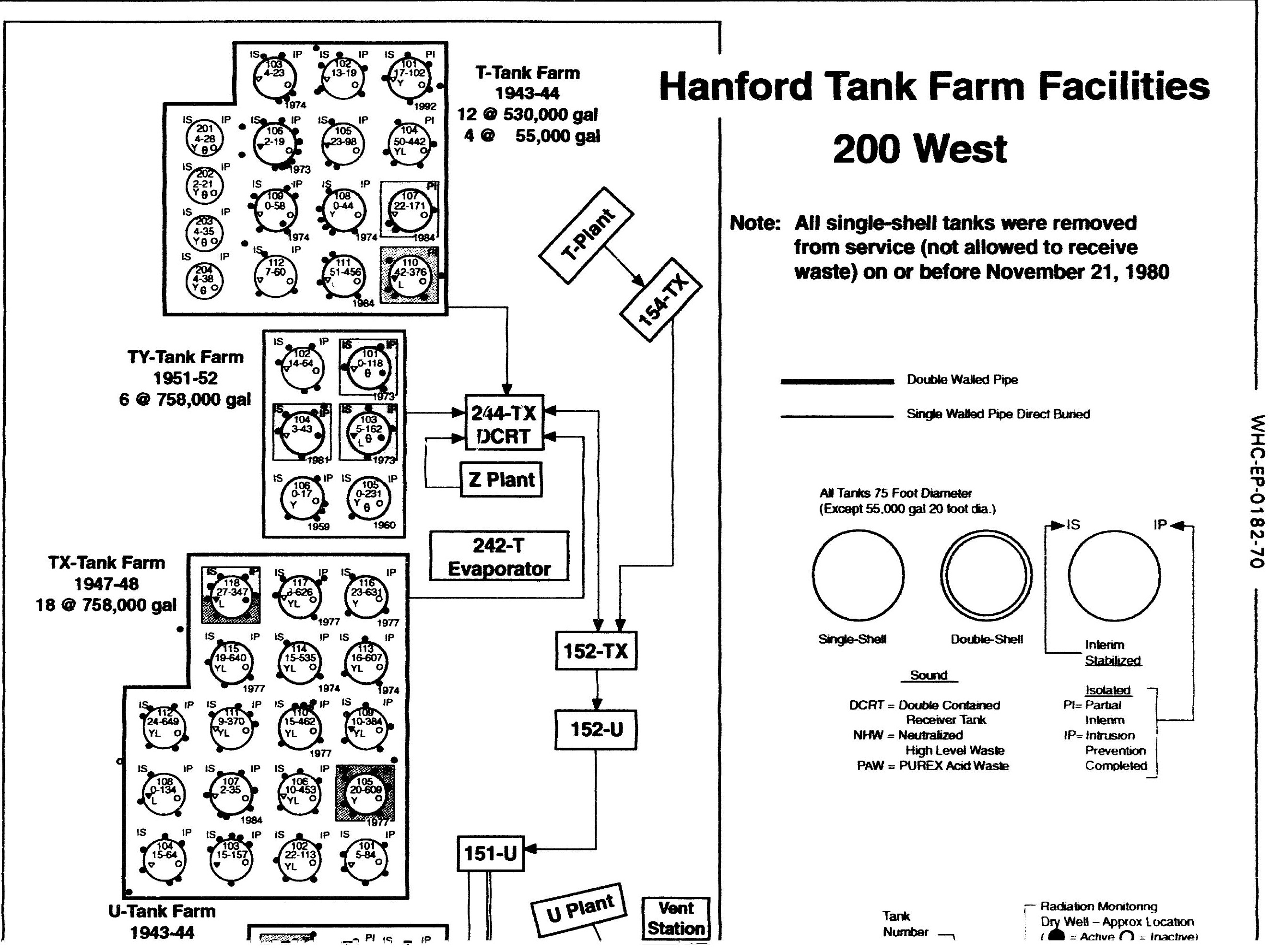


WHC-EP-0182-69

\section{APPENDIX E}

\section{MONTHLY SUMMARY}

TANK USE SUMMARY

INVENTORY SUMMARY BY TANK FARM INVENTORY AND STATUS BY TANK 
WHC-EP-0182-69

This page intentionally ?eft blank. 

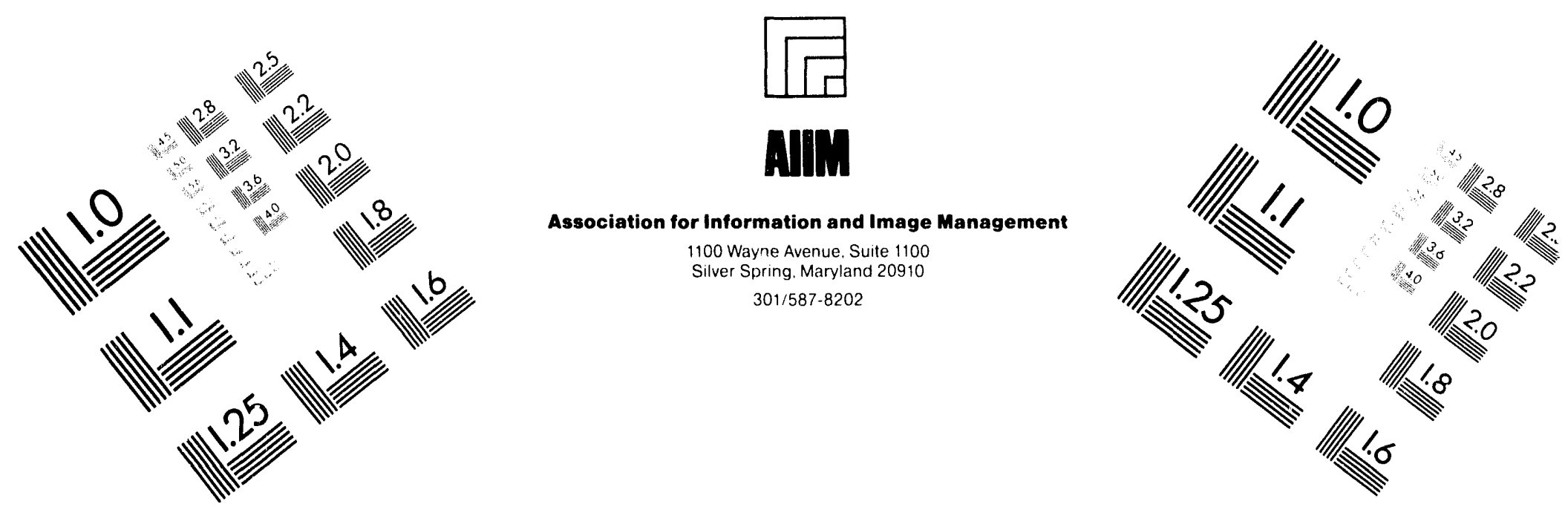

\section{Centimeter}

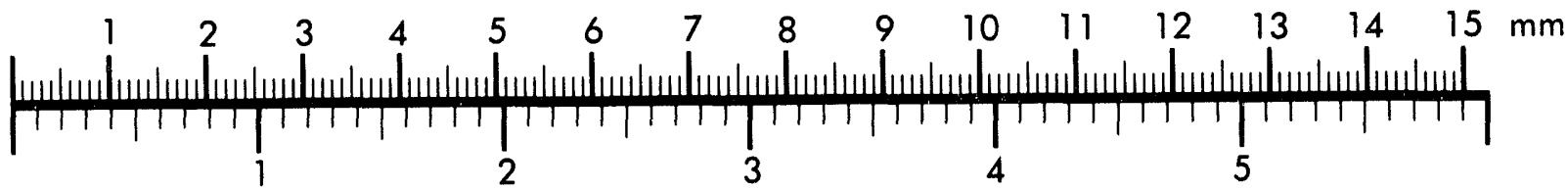
Inches
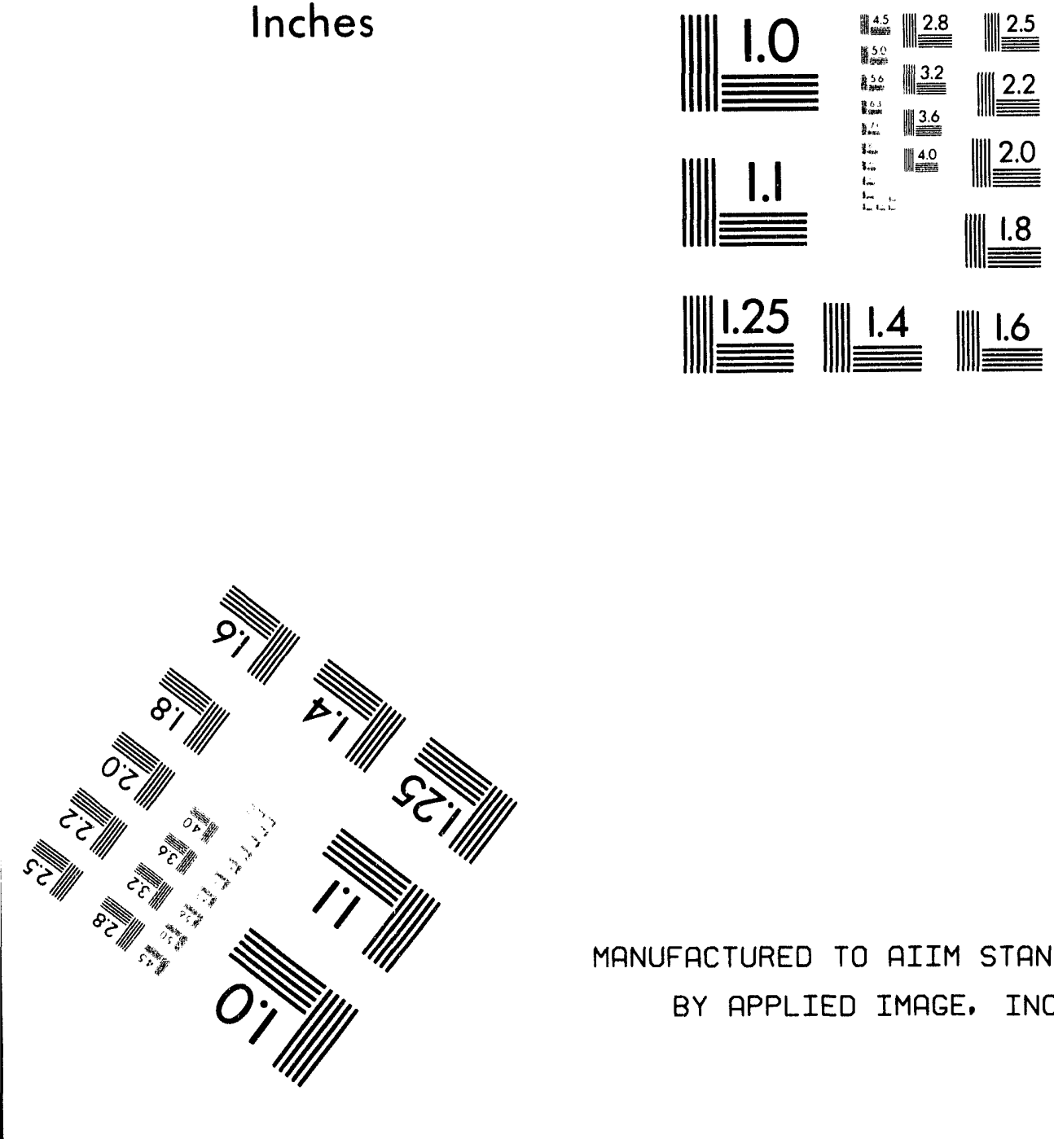

MANUFACTURED TO AIIM STANDARDS BY APPLIED IMAGE. INC.

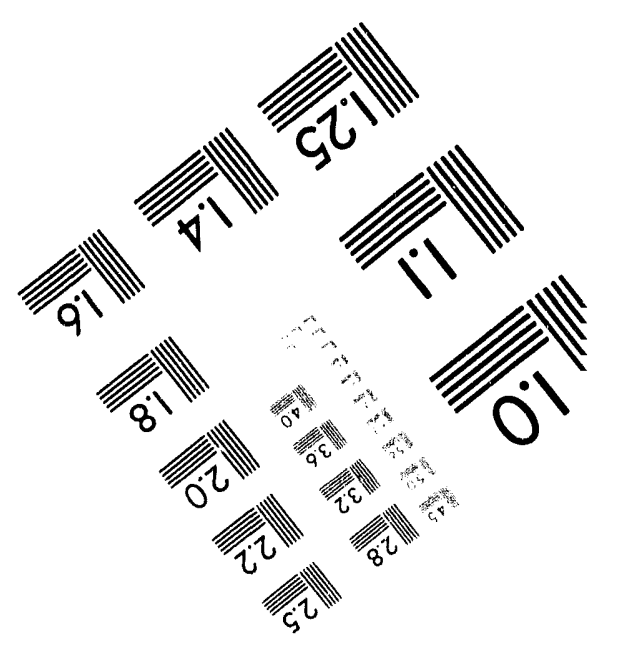



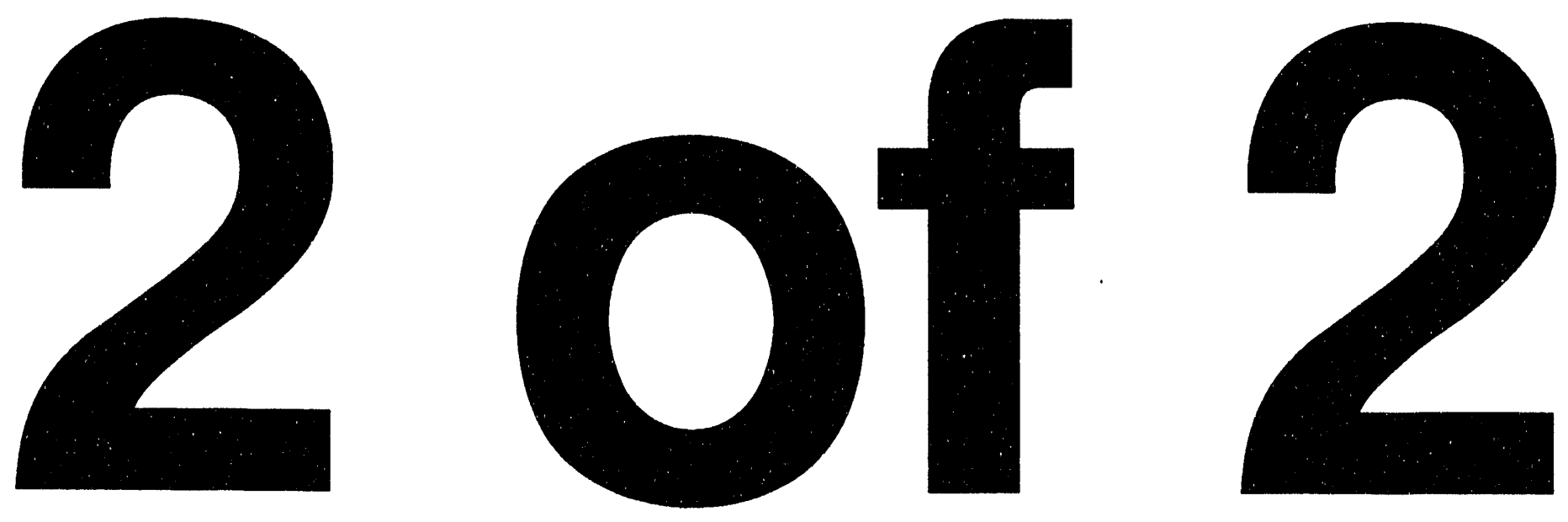


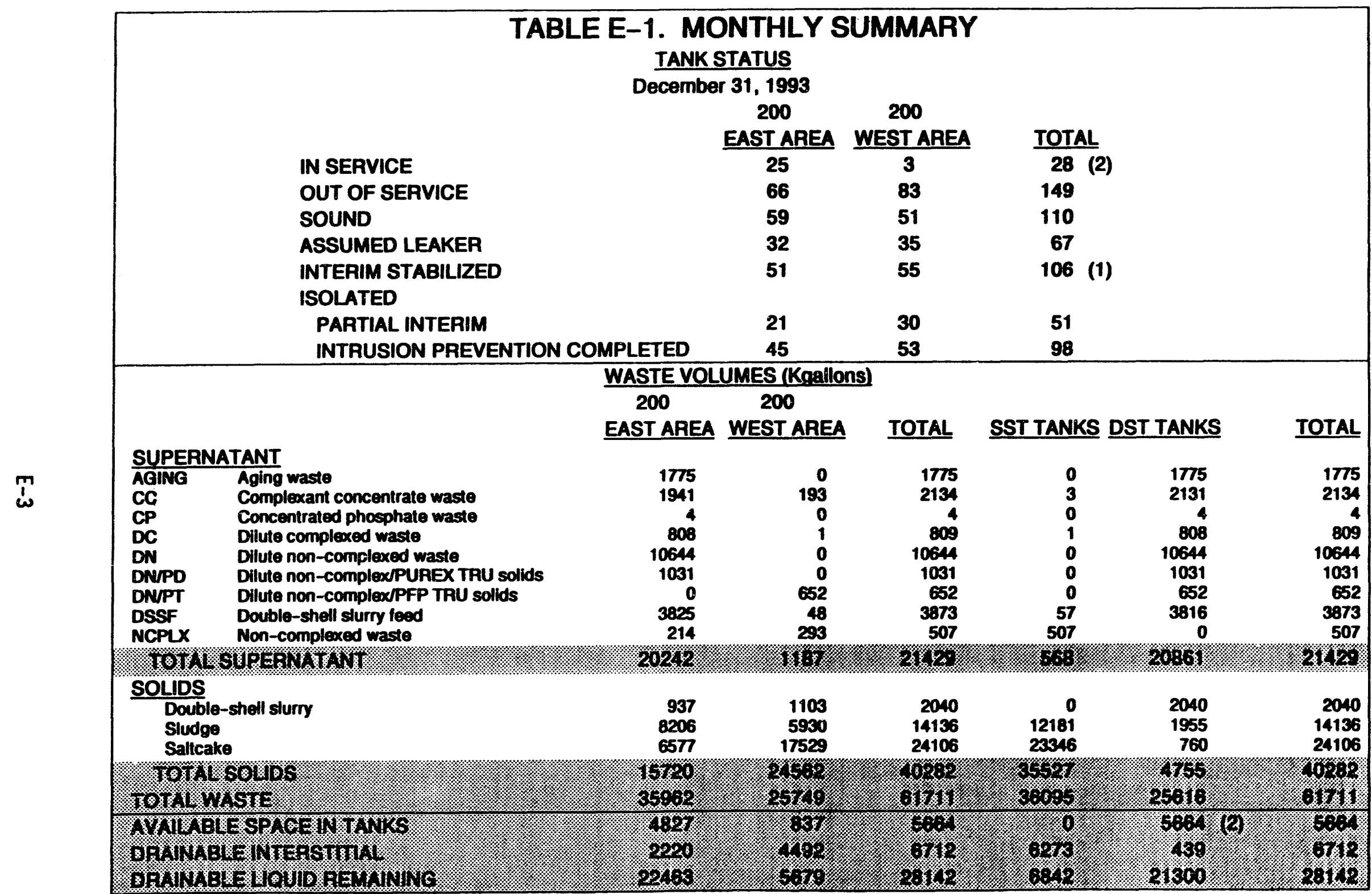

(1) Includes six tanks that do not meet current established supernatant and interstitial liquid stabillzation crteria, B-104, 110, 111, T-102, T-112, and U-110. (2) Includes stix double-shell tanks on Hydrogen Watch List not currently allowed to recelve wasto, 103-AN, 104-AN, 105-AN, 101-AW, 101-SY, and 103-SY. Note: +1- 1 Kgal differences are the result of computer rounding 
TABLE E-2. TANK USE SUMMARY

December 31, 1993

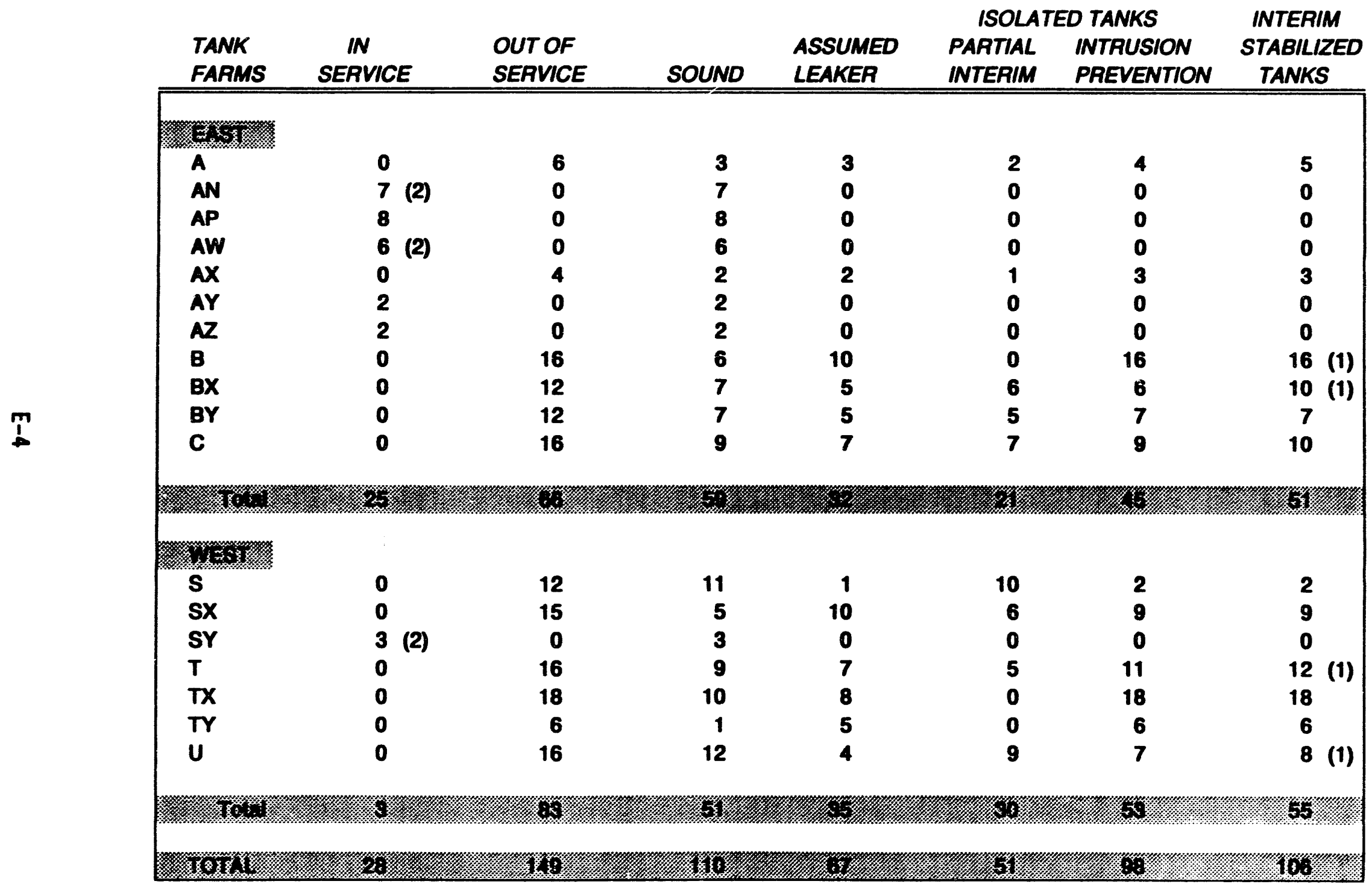

(1) Includes stx tanks that do not meet current established supernatant and interstitlal liquid stabilization criterla (B-104, 110, 111, T-102, 112. and U-110).

(2) Six Double-Shell Tanks on the Hydrogen Tank Watch List are not currently in service (AN-103, 104, 105, AW-101, SY-101 and 103). 
TABLE E-3. INVENTORY SUMMARY BY TANK FARM

WASTE VOLUMES (Kgallons)

December 31, 1993

SUPERNATANT LIQUID VOLUMES

SOLIDS VOLUME



Note: +1- $1 \mathrm{Kgal}$ differences are the result of computer rounding 
tABLE E-4. INVENTORY AMO STATUS BY TAMK DOUBLE-SHELL TAMKS

Decenber 31, 1993

TAMK STATUS

maste tank taMk

TAMK matl IMTEeRTY USE
LIOUID VoLune

DRAIN-

ECUIVA-

LEMT TOTAL AVAIL MATANT INTER- PLIPED

DRAIN- PUNP.

ABLE ABLE

LIOUID LIOUID

MSTE MASTE SPACE LIOUID STIT. IMCHES (Kgal) (Kgal)(Kgal) (Kgal) MONTH PUMPED (Kgal) (Kgal)
SOLIDS VOLLAE VOLUME DETERHIMATION

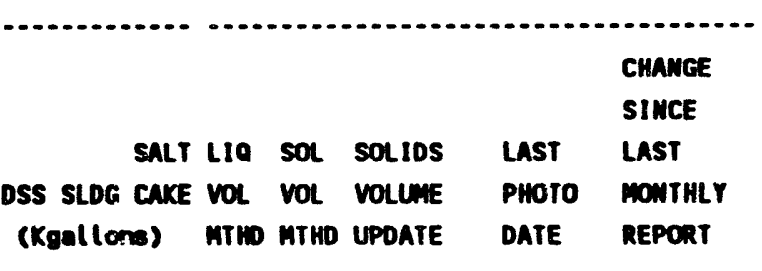

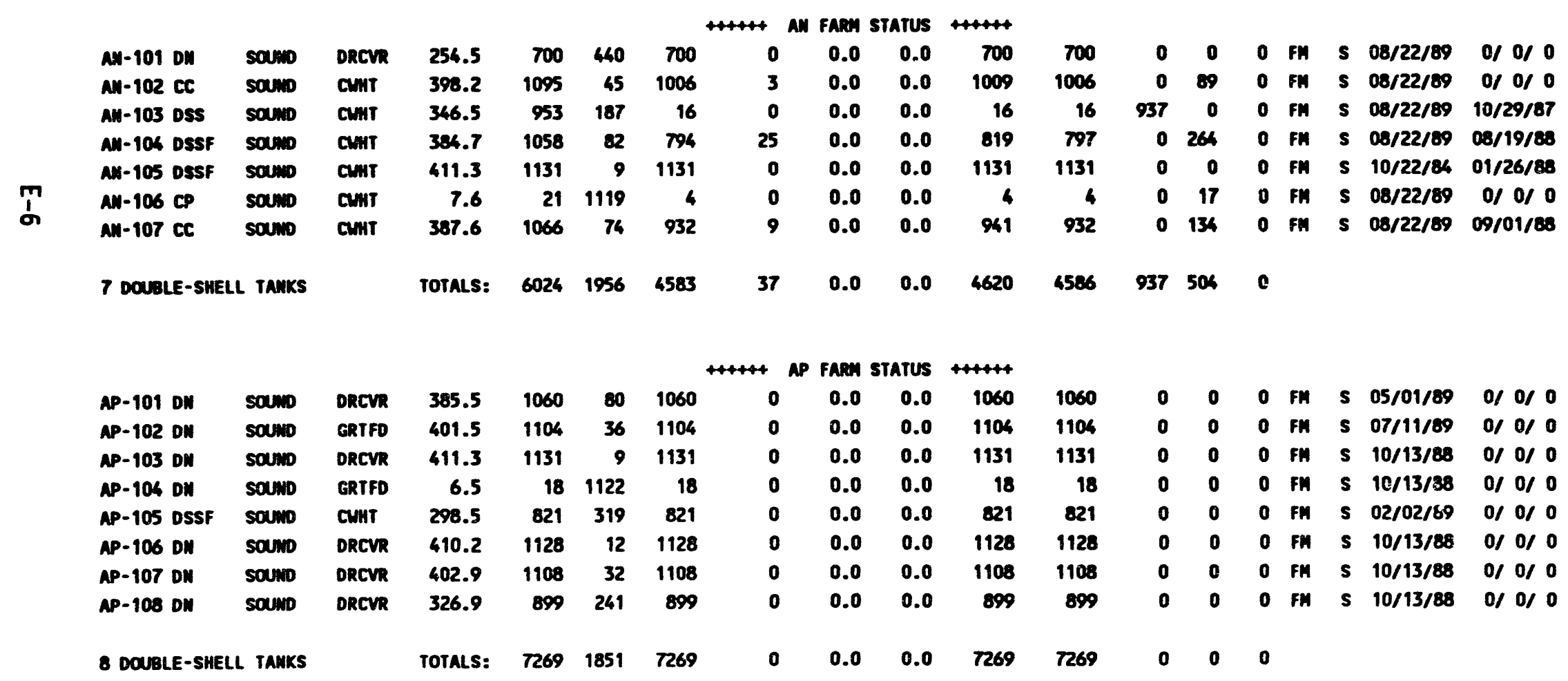


TABLE E-4. INVENTORY AND STATUS BY TANK DOUBLE-SHELL TANKS

Decenber 31, 1993

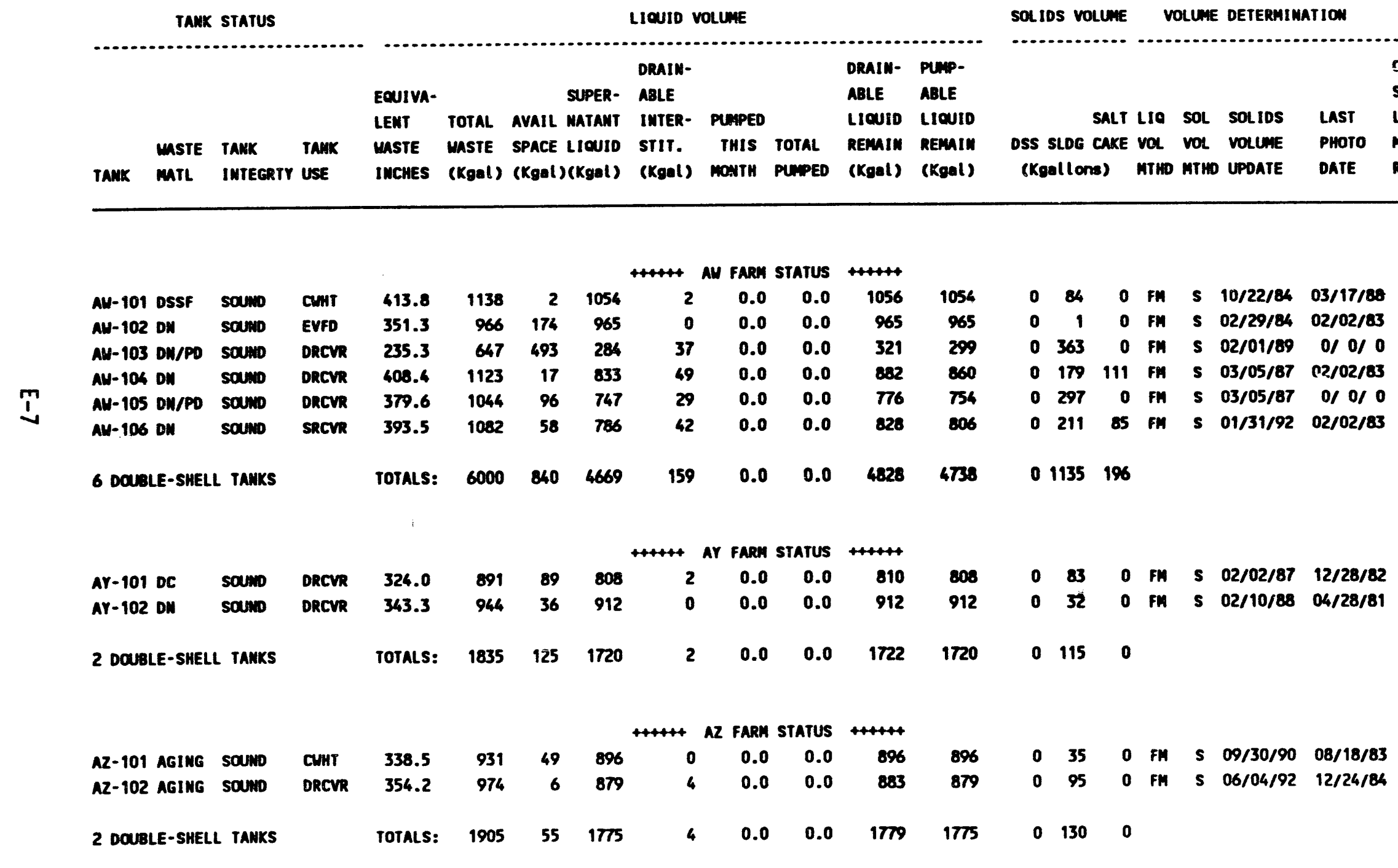


TABLE E-4. INVENTORY AND STATUS BY TANK DOUBLE-SHELL TAMKS

Decenber 31, 1993

TAMK STATUS

maste tank taMk

TAMK MTL INTECQTY USE
LIOUID VOLLAE

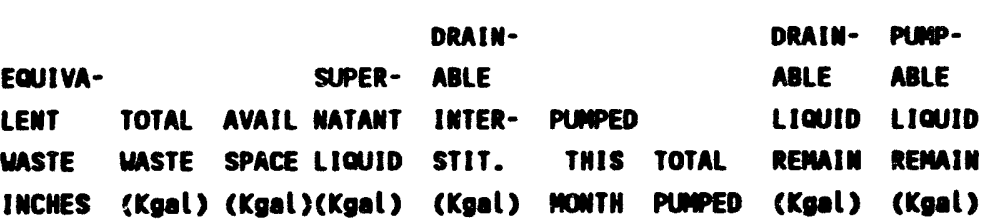

SOLIDS VOLUME VOLUHE DETERHIMATION

CHAMGE
DSS SLDE CAKE VOL VOL VOLUME PHOTO MONTHLY (Kgallons) MTHD MTHD UPDATE DATE REPORT

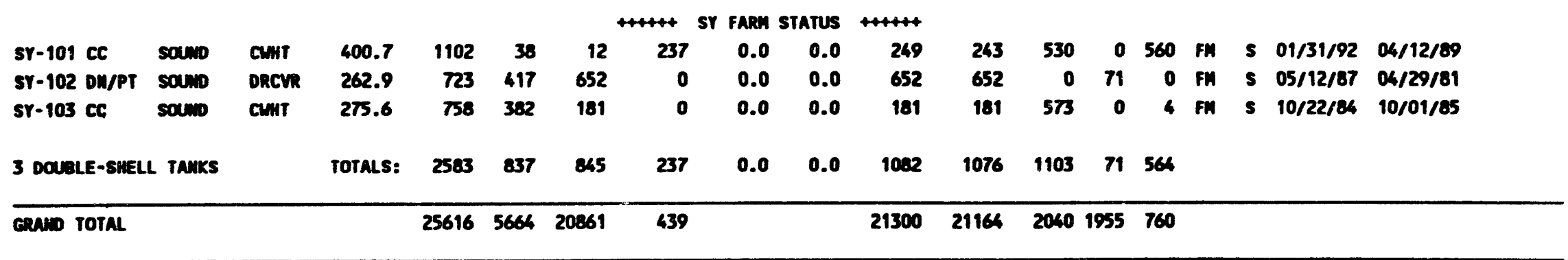

Mote: $+1-1$ kgal differences are the result of computer rounding

\begin{tabular}{|c|c|c|c|c|}
\hline \multirow[b]{2}{*}{ Tenk Farms } & \multirow{2}{*}{$\begin{array}{l}\text { Available Space } \\
\text { Calculations Used } \\
\text { In This Document } \\
\text { (Most Conservative) }\end{array}$} & \multicolumn{2}{|c|}{ Document $\mathrm{SD}-\mathrm{MH}-\mathrm{TI}-357$} & \multirow{2}{*}{$\begin{array}{c}\text { Document } \\
\text { OSD-T-151-00007we } \\
\text { Specification Limit }\end{array}$} \\
\hline & & Operating Limit & Tenk Cepecity & \\
\hline $\begin{array}{l}\text { AM, AP, AM, SY } \\
\text { AY, AZ (Aging Waste) }\end{array}$ & $\begin{array}{r}1,140,000 \mathrm{gal}(414.5 \mathrm{in.}) \\
980,000 \mathrm{gal}(356.4 \mathrm{in.})\end{array}$ & $\begin{array}{r}1,146,000 \text { gal (416 in.) } \\
990,000 \text { gal (360 in.) }\end{array}$ & $\begin{array}{l}1,160,000 \mathrm{gal}(421.8 \mathrm{in.}) \\
1,000,000 \mathrm{gal}(363.6 \mathrm{in.})\end{array}$ & $\begin{array}{l}1,160,500 \mathrm{gal}(422 \mathrm{in.}) \\
1,001,000 \mathrm{gal}(364 \mathrm{in.})\end{array}$ \\
\hline
\end{tabular}

- WHC-SD-MM-TI-357, "Waste Storage Tank Status and Leak Detection Criteria."

*HAHC-OSD-T-151-00007, mopereting Specificotions for 241-AN, AP, AH, AY, A2, E SY Tank farms." 
TABLE E-5. INVENTORY AMD STATUS BY TAMK

SINGLE-SHELL TAMKS

Decenber 31, 1993

\begin{tabular}{|c|c|c|c|c|c|c|c|c|c|c|c|c|c|c|c|c|c|c|}
\hline & TANK 5 & TATUS & & & & LIOUID & VOLUATE & & & & SOLIDS & VOLUNE & & VOLU & E DETERM & IMATIOW & & \\
\hline & $\begin{array}{l}\text { MASTE } \\
\text { MATERIAL }\end{array}$ & $\begin{array}{l}\text { TANK } \\
\text { IMTECRITY }\end{array}$ & $\begin{array}{l}\text { STABIL/ } \\
\text { I SOLATION } \\
\text { STATUS }\end{array}$ & $\begin{array}{r}\text { TOTAL } \\
\text { MAste } \\
\text { (Kgal) }\end{array}$ & $\begin{array}{l}\text { SUPER- } \\
\text { MATAMT } \\
\text { LIOUID } \\
\text { (Kgal) }\end{array}$ & $\begin{array}{l}\text { ORAIN- } \\
\text { ABLE } \\
\text { INTER- } \\
\text { STIT. } \\
\text { (Kgal) }\end{array}$ & $\begin{array}{l}\text { PUNPED } \\
\text { THIS } \\
\text { MONTH } \\
\text { (Kgal) }\end{array}$ & $\begin{array}{l}\text { TOTAL } \\
\text { PUMPED } \\
\text { (Kgal) }\end{array}$ & $\begin{array}{l}\text { DRAIN- } \\
\text { ABLE } \\
\text { LIOUID } \\
\text { REMAII } \\
\text { (Kgal) }\end{array}$ & $\begin{array}{l}\text { PLIPP- } \\
\text { ABLE } \\
\text { LIQUID } \\
\text { REMAIM } \\
\text { (Kgal) }\end{array}$ & $\begin{array}{l}\text { SLUDGE } \\
\text { (Kgal) }\end{array}$ & $\begin{array}{l}\text { SALTCAKE } \\
\text { (Kgal) }\end{array}$ & $\begin{array}{l}\text { LICUIDS } \\
\text { VOLUNE } \\
\text { METHOD }\end{array}$ & $\begin{array}{l}\text { SOLIDS } \\
\text { VOLUAE } \\
\text { METHOD }\end{array}$ & $\begin{array}{l}\text { SOLIDS } \\
\text { VOLUAEE } \\
\text { UPDATE }\end{array}$ & $\begin{array}{l}\text { SOLIDS } \\
\text { UPOATE } \\
\text { SOURCE } \\
\text { SEE } \\
\text { FOOTNOTE }\end{array}$ & $\begin{array}{l}\text { LAST } \\
\text { PHOTO } \\
\text { DATE }\end{array}$ & $\begin{array}{l}\text { CHG } \\
\text { SIMCE } \\
\text { LAST } \\
\text { MNTHLY } \\
\text { REPORT }\end{array}$ \\
\hline
\end{tabular}

\begin{tabular}{|c|c|c|c|c|c|c|c|c|c|c|c|c|c|c|c|c|c|}
\hline & & & & & & +++ & A FARM 5 & Tatus & t+t+t+t & & & & & & & & \\
\hline A-101 & DSSF & sous & IPI & 953 & 0 & 413 & 0.0 & 0.0 & 413 & 390 & 3 & 950 & $p$ & $\mathbf{F}$ & $11 / 21 / 80$ & & $08 / 21 / 85$ \\
\hline$A-102$ & DSSF & sound & IS/PI & 41 & 4 & 2 & 0.0 & 39.5 & 6 & 0 & 15 & 22 & $\mathbf{P}$ & $\mathbf{F P}$ & $07 / 27 / 89$ & (1) & $07 / 20 / 89$ \\
\hline$A-103$ & DSSF & ASID LKR & IS/IP & 371 & 5 & 15 & 0.0 & 111.0 & 20 & 0 & 366 & 0 & - & FP & $06 / 03 / 88$ & (1) & $12 / 28 / 88$ \\
\hline$a-104$ & nCPLX & ASED LKR & IS/IP & 28 & 0 & 0 & 0.0 & 0.0 & 0 & 0 & 28 & 0 & $m$ & PS & $01 / 27 / 78$ & & $06 / 25 / 86$ \\
\hline$A-105$ & MCPLX & ASW LKR & IS/IP & 19 & 0 & 4 & 0.0 & 0.0 & 4 & 0 & 19 & 0 & $p$ & $m$ & $08 / 23 / 79$ & (1) & $08 / 20 / 86$ \\
\hline$A-106$ & CP & sound & IS/IP & 125 & 0 & 7 & 0.0 & 0.0 & 7 & 0 & 125 & 0 & $\mathbf{P}$ & $\boldsymbol{n}$ & $09 / 07 / 82$ & & $08 / 17 / 86$ \\
\hline \multirow[t]{2}{*}{6 SIMEL } & LE-SHELL & TANKS & TOTALS & 1537 & 9 & 441 & 0.0 & 150.5 & 450 & 390 & 556 & 972 & & & & & \\
\hline & & & & & \multicolumn{2}{|c|}{$+++++t$} & aX FARH & STATUS & $+++++t$ & & & & & & & & \\
\hline$a x-101$ & DSSF & souid & IPI & 748 & 0 & 320 & 0.0 & 0.0 & 320 & 298 & 3 & 745 & $P$ & $\mathbf{F}$ & $05 / 06 / 82$ & & $08 / 18 / 87$ \\
\hline$a x-102$ & cc & ASID LKR & IS/IP & 39 & 3 & 14 & 0.0 & 13.0 & 17 & 3 & 7 & 29 & $\mathbf{F}$ & $\mathbf{s}$ & $09 / 06 / 88$ & & $06 / 05 / 89$ \\
\hline$N x-103$ & cc & soum & IS/IP & 112 & o & 36 & 0.0 & 0.0 & 36 & 3 & 2 & 110 & $\mathbf{F}$ & $\mathbf{s}$ & $08 / 19 / 87$ & & $08 / 13 / 87$ \\
\hline$N x-104$ & MCPLX & ASMD LKR & IS/IP & 7 & 0 & 0 & 0.0 & 0.0 & 0 & 0 & 7 & 0 & P & $\boldsymbol{n}$ & $04 / 28 / 82$ & & $08 / 18 / 87$ \\
\hline 4 SIMGL & LE-SHELL & TANKS & TOTALS: & 906 & 3 & 370 & 0.0 & 13.0 & 373 & 304 & 19 & 884 & & & & & \\
\hline
\end{tabular}


TABLE E-5. INVENTORY AMD STATUS BY tAMK SIMGLE-SHELL TAMKS

Decentor 31, 1993

TaMK STATUS

UASTE TAMK

MASTE TAMK

$$
\text { STABIL/ }
$$

isolatio

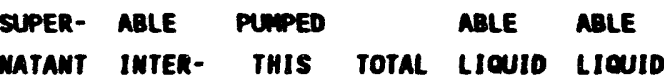

DRAIN- PUIP-

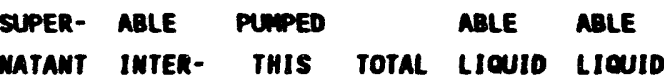

SOLIDS volune

solids volure

SLUDEE SALTCAKE
(Kgal) (Kgal)

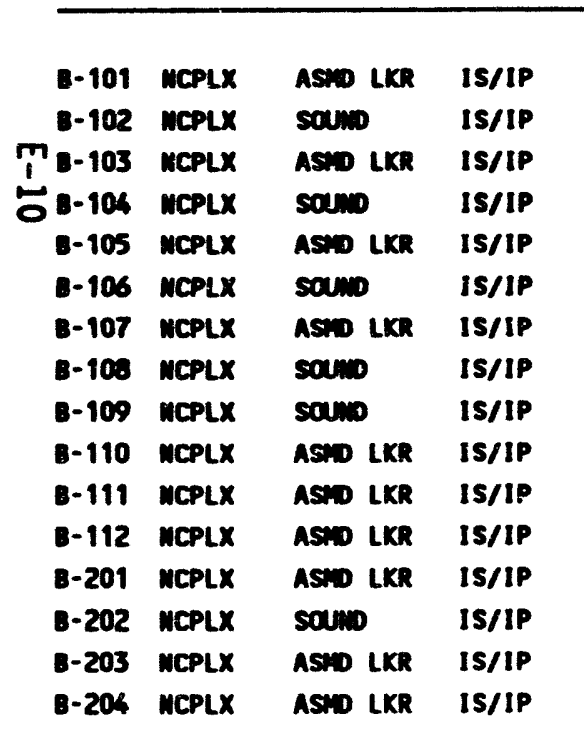

20

$\begin{array}{ll}113 & 0 \\ 32 & 6 \\ 59 & 0\end{array}$

371

371
306
117

117

165

127

246

237

33

29

27

51
50

50

2057

$\begin{array}{ll}0.0 & 0.0 \\ 0.0 & 0.0\end{array}$

$\begin{array}{ll}0.0 & 0.0 \\ 0.0 & 0.0\end{array}$

0.0

0.00 .0

$0.0 \quad 0.0$

$0.0 \quad 0.0$

$0.0 \quad 0.0$

$0.0 \quad 0.0$

0.00 .0

$0.0 \quad 0.0$

$\begin{array}{ll}0.0 & 0.0 \\ 0.0 & 0.0\end{array}$

$0.0 \quad 0.0$

0.00 .0

$0.0 \quad 0.0$

0.00 .0

$0.0 \quad 0.0$

6000

6
4
6

47
23

7

7
13
4

4
8
23

23

3

4

3
6
6

179

$\begin{array}{rr}0 & 113 \\ 0 & 18\end{array}$

58

59
301

301
40

116

164

96

127
245

236
30

30

28

50

49

$\begin{array}{rr}0 & 0 \\ 47 & 60\end{array}$

VOLUME DETERMIMATION

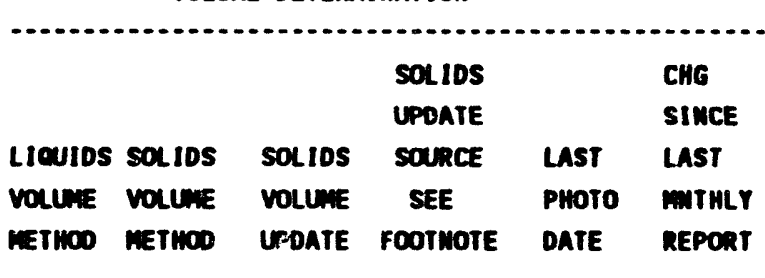


TABLE E-5. IIVENTORY AND STATUS BY TANK

SIMGLE-SHELL TAMKS

Decentber 31, 1993

\begin{tabular}{|c|c|c|c|c|c|c|c|c|c|c|c|c|c|c|c|c|c|c|}
\hline & TAMK $S$ & tatus & & & & LIOUIO & VOLUAE & & & & SOLIDS & VOLUNE & - & OLL & DETERM & IMATION & & \\
\hline A AKK & $\begin{array}{l}\text { MASTE } \\
\text { MATERIAL }\end{array}$ & $\begin{array}{l}\text { TANK } \\
\text { INTEGRITY }\end{array}$ & $\begin{array}{l}\text { STABIL/ } \\
\text { ISOLATION } \\
\text { STATUS }\end{array}$ & $\begin{array}{l}\text { TOTAL } \\
\text { WASTE } \\
\text { (Kgal) }\end{array}$ & $\begin{array}{l}\text { SUPER- } \\
\text { MATANT } \\
\text { LIOUID } \\
\text { (Kgal) }\end{array}$ & $\begin{array}{l}\text { DRAIN- } \\
\text { ABLE } \\
\text { INTER- } \\
\text { STIT. } \\
\text { (Kgal) }\end{array}$ & $\begin{array}{l}\text { PUAPED } \\
\text { THIS } \\
\text { HONTH } \\
\text { (Kgal) }\end{array}$ & $\begin{array}{l}\text { TOTAL } \\
\text { PUMPED } \\
\text { (Kgal) }\end{array}$ & $\begin{array}{l}\text { DRAIN- } \\
\text { ABLE } \\
\text { LIOUIO } \\
\text { REMaIN } \\
\text { (Kgal) }\end{array}$ & $\begin{array}{l}\text { PUMP- } \\
\text { ABLE } \\
\text { LIOUID } \\
\text { REMAII } \\
\text { (Kgal) }\end{array}$ & $\begin{array}{l}\text { SLLDGE } \\
\text { (Kgal) }\end{array}$ & $\begin{array}{l}\text { SALTCAKE } \\
\text { (Kgal) }\end{array}$ & $\begin{array}{l}\text { LICUIDS } \\
\text { VoLUNE } \\
\text { METHOD }\end{array}$ & $\begin{array}{l}\text { SOLIDS } \\
\text { VOLUAEE } \\
\text { METHOD }\end{array}$ & $\begin{array}{l}\text { SOLIDS } \\
\text { VOLUWE } \\
\text { UPOATE }\end{array}$ & $\begin{array}{l}\text { SOLIDS } \\
\text { UPDATE } \\
\text { SOURCE } \\
\text { SEE } \\
\text { FOOTMOTE }\end{array}$ & $\begin{array}{l}\text { LAST } \\
\text { PHOTO } \\
\text { DATE }\end{array}$ & $\begin{array}{l}\text { CHG } \\
\text { SINCE } \\
\text { LAST } \\
\text { MUTHLY } \\
\text { REPORT }\end{array}$ \\
\hline
\end{tabular}

\begin{tabular}{|c|c|c|c|c|c|c|c|c|c|c|c|c|c|c|c|c|}
\hline & & & & & $+++8 x$ & FARM & status & $t+++t+$ & & & & & & & & \\
\hline $8 x-101$ NCPLX & ASID LKR & IS/IP & 43 & 1 & 0 & 0.0 & 0.0 & 1 & 0 & 42 & 0 & $\mathbf{P}$ & $\boldsymbol{M}$ & $04 / 28 / 82$ & & $11 / 24 / 88$ \\
\hline $8 x-102$ MCPLX & ASID LKR & IS/IP & 96 & 0 & 4 & 0.0 & 0.0 & 4 & 0 & 96 & 0 & $\mathbf{P}$ & H & $04 / 28 / 82$ & & $09 / 18 / 85$ \\
\hline$B X-103$ NCPLX & SOUnd & IS/IP & 66 & 4 & $\mathbf{0}$ & 0.0 & 0.0 & 4 & 0 & 52 & 0 & $\mathbf{P}$ & $\mathbf{F}$ & $11 / 29 / 83$ & & $10 / 31 / 86$ \\
\hline $8 x-104$ NCPLX & SOUnd & IS/IP & 99 & 3 & 30 & 0.0 & 17.4 & 33 & 27 & 96 & 0 & $F$ & $\mathbf{F}$ & $09 / 22 / 89$ & (1) & $09 / 21 / 89$ \\
\hline $8 x-105 N C P L X$ & soumd & IS/IP & 51 & 5 & 6 & 0.0 & 15.0 & 11 & 4 & 43 & 3 & $F$ & $\mathbf{s}$ & 09/0ิ5iةó & (1) & $10 / 23 / 86$ \\
\hline $8 x-106$ NCPLX & sound & $|P|$ & 46 & 15 & 0 & 0.0 & 0.0 & 15 & 15 & 31 & 0 & MP & PS & $04 / 28 / 82$ & & $05 / 19 / 88$ \\
\hline$B x-107$ MCPLX & SOUnd & $1 S / P I$ & 345 & 1 & 29 & 0.0 & 23.1 & 30 & 23 & 344 & 0 & $\mathbf{M P}$ & $\mathbf{P}$ & $09 / 18 / 90$ & (2) & $09 / 11 / 90$ \\
\hline $8 x-108$ NCPLX & ASID LKR & $15 / 1 P$ & 26 & 0 & 1 & 0.0 & 0.0 & 1 & 0 & 26 & 0 & 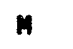 & PS & $07 / 31 / 79$ & (1) & $10 / 23 / 86$ \\
\hline BX-109 MCPLA & SOUND & IS/PI & 193 & 0 & 13 & 0.0 & 8.2 & 13 & 8 & 193 & 0 & FP & $\mathbf{P}$ & $09 / 17 / 90$ & (2) & $09 / 11 / 90$ \\
\hline$B X-110$ MCPLX & ASID LKR & IS/PI & 198 & 0 & 15 & 4.0 & 4.0 & 17 & 6 & 189 & 9 & $\operatorname{MP}$ & $\boldsymbol{M}$ & $08 / 22 / 85$ & (1) & $07 / 31 / 85$ \\
\hline $8 x-111$ NCPLX & ASMD LKR & /PI & 211 & 0 & 15 & 23.7 & 54.7 & 15 & 0 & 68 & 143 & $M$ & M & $07 / 26 / 77$ & & $07 / 16 / 93$ \\
\hline BX-112 MCPLXX & sourd & $|S / P|$ & 165 & 1 & 7 & 0.0 & 4.1 & 8 & 2 & 164 & 0 & FP & $\mathbf{P}$ & $09 / 17 / 90$ & (2) & $09 / 11 / 90$ \\
\hline 12 SIMGLE-SHELL & TANKS & TOTALS: & 1539 & 30 & 120 & 27.7 & 126.5 & 151 & 85 & 1354 & 155 & & & & & \\
\hline & & & & & $+++B Y$ & FARH & STATUS & +t+t+t+ & & & & & & & & \\
\hline BY-101 MCPLX & sound & IS/IP & 387 & 0 & 5 & 0.0 & 35.8 & 5 & 0 & 109 & 278 & $p$ & $\boldsymbol{n}$ & $05 / 30 / 84$ & & $09 / 19 / 89$ \\
\hline BY-102 NCPLX & souno & /PI & 341 & 0 & 41 & 0.0 & 123.3 & 41 & 22 & 0 & 341 & MP & $n$ & $08 / 30 / 91$ & (2) & $09 / 11 / 87$ \\
\hline BY-103 NCPLX & ASND LKR & /PI & 400 & 0 & 160 & 0.0 & 78.5 & 160 & 137 & 5 & 395 & MP & $M$ & $04 / 03 / 90$ & (2) & $09 / 07 / 89$ \\
\hline BY-104 MCPLX & scound & IS/IP & 406 & 0 & 18 & 0.0 & 329.5 & 18 & 0 & 40 & 366 & $\mathbf{P}$ & $n$ & $04 / 28 / 82$ & & $04 / 27 / 83$ \\
\hline $8 Y-105$ MCPLX & ASTD LKR & /PI & 503 & 0 & 192 & 0.0 & 0.0 & 192 & 169 & 44 & 459 & $\mathbf{p}$ & $\operatorname{mP}$ & $04 / 28 / 82$ & & $07 / 11 / 86$ \\
\hline BY-106 NCPLX & ASID LKR & |PI & 642 & 0 & 235 & 0.0 & 0.0 & 235 & 213 & 95 & 547 & p & MP & $04 / 28 / 82$ & & $11 / 04 / 82$ \\
\hline BY-107 NCPLX & ASTD LKR & $15 / 1 P$ & 266 & 0 & 25 & 0.0 & 56.4 & 25 & 0 & 60 & 206 & $\mathbf{P}$ & MP & $04 / 28 / 82$ & & $10 / 15 / 86$ \\
\hline
\end{tabular}


TABLE E-5. IMVENTORY ANO STATUS BY TAMK SINGLE-SHELL TANKS

Decenber 31, 1993

TAMK status

(1)

DRAIN-
SUPER- ABLE

LIOUID Volune

STABIL TOTAL MATAMT IMTER- THIS TOTAL LIOUID LIOUID

MASTE TAMK ISOLATION MASTE LJOUID STIT. NONTH PUMPED REMIII REMIN TAMK MATERIAL IMTEGRITY STATUS (Kgal) (Kgal) (Kgol) (Kgal) (Kgal) (Kgal) (Kgal)
SOLIDS Voune

c..............

ne

\begin{tabular}{|c|c|c|c|c|c|}
\hline & & & SOLLIDS & & CHG \\
\hline & & & UPOATE & & SIMCE \\
\hline $\begin{array}{l}\text { LIOUIOS } \\
\text { VOLUIE } \\
\text { METHOD }\end{array}$ & $\begin{array}{l}\text { Solios } \\
\text { Volune } \\
\text { METMOD }\end{array}$ & $\begin{array}{l}\text { SOLIDS } \\
\text { VOLUARE } \\
\text { UPOATE }\end{array}$ & $\begin{array}{l}\text { sounce } \\
\text { seE } \\
\text { Footwote }\end{array}$ & $\begin{array}{l}\text { LAST } \\
\text { PHoto } \\
\text { DATE }\end{array}$ & $\begin{array}{l}\text { LAST } \\
\text { MATMLT } \\
\text { REPORT }\end{array}$ \\
\hline
\end{tabular}

SLLDEE SALTCAKE (Keal) (Koal)

\begin{tabular}{|c|c|}
\hline BY-100 MCPLX & ASND LKR \\
\hline Br-109 MCPLX & soum \\
\hline BY-110 MCPLX & sound \\
\hline $\begin{array}{l}\text { BY-111 } \text { NCPLX } \\
\text { Br-112 } \text { जCPLX }\end{array}$ & $\begin{array}{l}\text { soum } \\
\text { sotin }\end{array}$ \\
\hline
\end{tabular}

IS/IP
IPI
IS/IP
IS/IP
IS/IP

12 SINGLE-SHELL TANKS

Torals:

4744

$\begin{array}{ll}228 & 0 \\ 423 & 0 \\ 396 & 0 \\ 459 & 0 \\ 291 & 0\end{array}$

90.0

$\begin{array}{rrrr}0.0 & 27.5 & 9 & 0 \\ 0.0 & 93.5 & 76 & 57\end{array}$

$\begin{array}{ll}0.0 & 213.3 \\ 0.0 & 313.2\end{array}$

$\begin{array}{rr}7 & 57 \\ 0 & 0\end{array}$

8

0.0116 .4

200

$\begin{array}{rrlllll}154 & 74 & \text { M } & \text { M } & 04 / 28 / 82 & 10 / 15 / 86 \\ 83 & 340 & \text { F } & \text { PS } & 08 / 30 / 91 & \text { (2) } & 10 / 15 / 86 \\ 103 & 295 & \text { M } & \text { S } & 09 / 10 / 79 & 07 / 26 / 84 \\ 21 & 438 & \text { P } & \text { M } & 04 / 28 / 82 & 10 / 31 / 86 \\ 5 & 286 & \text { P } & \text { M } & 04 / 28 / 82 & 04 / 14 / 88 \\ 719 & 4025 & & & & & \end{array}$

\begin{tabular}{|c|c|c|c|c|c|c|c|c|c|c|c|c|c|c|c|c|c|}
\hline$c-101$ & $\operatorname{McP} L x$ & ASN LKR & IS/IP & 88 & 0 & 3 & 0.0 & 0.0 & 3 & 0 & 88 & 0 & H & M & $11 / 29 / 83$ & & $11 / 17 / 87$ \\
\hline$c-102$ & DC & soum & IPI & 623 & 0 & 37 & 0.0 & 11.6 & 37 & 19 & 423 & 0 & $F$ & FP & $04 / 28 / 82$ & & $05 / 18 / 76$ \\
\hline$c-103$ & MCPLX & soum & IPI & 195 & 133 & 0 & 0.0 & 0.0 & 133 & 133 & 62 & 0 & $\mathbf{F}$ & $s$ & $10 / 22 / 90$ & (2) & $07 / 28 / 87$ \\
\hline$c-104$ & $c c$ & souno & IS/IP & 295 & 0 & 11 & 0.0 & 0.0 & 11 & 5 & 295 & 0 & FP & $\mathbf{P}$ & $09 / 22 / 89$ & (1) & $07 / 25 / 90$ \\
\hline$c-105$ & nCPLX & souno & IPI & 150 & 0 & 11 & 0.0 & 0.0 & 11 & 4 & 150 & 0 & $F$ & $\mathbf{s}$ & $05 / 31 / 85$ & & $04 / 01 / 80$ \\
\hline$c-106$ & MCPLX & sound & /PI & 229 & 32 & 16 & 0.0 & 0.0 & 48 & 42 & 197 & 0 & $\mathbf{F}$ & PS & $04 / 28 / 82$ & & $04 / 05 / 79$ \\
\hline$c-107$ & DC & Sounto & IPI & 275 & 0 & 26 & 0.0 & 16.3 & 26 & 20 & 275 & 0 & $\mathbf{F}$ & $\mathbf{s}$ & $01 / 30 / 92$ & (2) & $00 / 00 / 00$ \\
\hline$c-108$ & MCPLX & soum & IS/IP & 66 & 0 & 0 & 0.0 & 0.0 & 0 & $\mathbf{0}$ & 66 & 0 & $n$ & $\mathbf{s}$ & $02 / 24 / 84$ & (1) & $12 / 05 / 74$ \\
\hline$c-109$ & MCPLX & soum & IS/IP & 66 & 4 & 0 & 0.0 & 0.0 & 4 & 0 & 62 & 0 & $M$ & PS & $11 / 29 / 83$ & & $01 / 30 / 76$ \\
\hline$c-110$ & DC & ASWD LKR & $|P|$ & 187 & 0 & 7 & 0.0 & 8.9 & 7 & 5 & 187 & 0 & $\mathbf{F}$ & FMP & $03 / 01 / 92$ & (2) & $08 / 12 / 86$ \\
\hline$c-111$ & MCPLX & ASID LKR & IS/IP & 57 & 0 & 0 & 0.0 & 0.0 & 0 & 0 & 57 & 0 & $n$ & $s$ & $04 / 28 / 82$ & & $02 / 25 / 70$ \\
\hline$c-112$ & MCPLX & sound & IS/PI & 104 & 0 & 32 & 0.0 & 0.0 & 32 & 26 & 104 & 0 & $\boldsymbol{M}$ & PS & $09 / 18 / 90$ & (2) & $09 / 18 / 90$ \\
\hline
\end{tabular}


TABLE E-5. INYENTORY AND STATUS BY TAMK

SIMGLE-SHELL TAMKS

Decenber 31, 1993

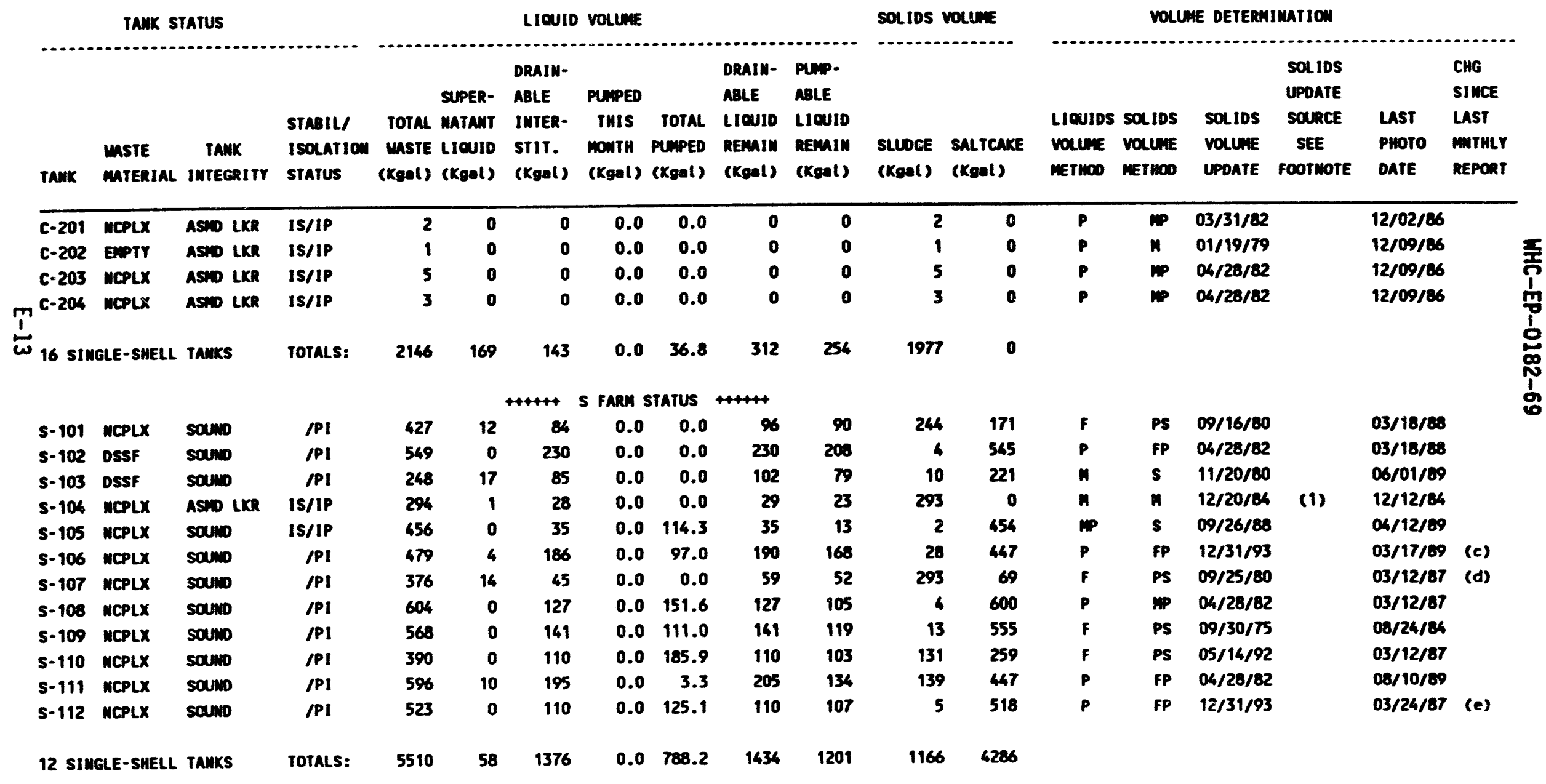




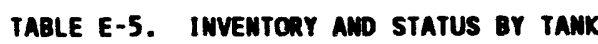
SIMGLE-SHELL TAMKS Decenber 31, 1993

\begin{tabular}{|c|c|c|c|c|c|c|c|c|c|c|c|c|c|c|c|c|c|c|}
\hline \multirow[b]{2}{*}{ TAMK } & \multicolumn{2}{|c|}{ TANK status } & \multicolumn{8}{|c|}{ LIOUID VOLUME } & \multicolumn{2}{|c|}{ SOLIDS VOLLANE } & \multicolumn{5}{|c|}{ VOLUME DETERMIMATION } & \\
\hline & $\begin{array}{l}\text { MASTE } \\
\text { MATERIAL }\end{array}$ & $\begin{array}{c}\text { TANK } \\
\text { INTEGRITY }\end{array}$ & $\begin{array}{l}\text { STABIL/ } \\
\text { I SOLATION } \\
\text { STATUS }\end{array}$ & $\begin{array}{l}\text { TOTAL } \\
\text { MASTE } \\
\text { (Kgal) }\end{array}$ & $\begin{array}{l}\text { SUPER- } \\
\text { MATANT } \\
\text { LIOUID } \\
\text { (Kgal) }\end{array}$ & $\begin{array}{l}\text { DRAIN- } \\
\text { ABLE } \\
\text { INTER- } \\
\text { STIT. } \\
\text { (Kgal) }\end{array}$ & $\begin{array}{l}\text { PUMPED } \\
\text { THIS } \\
\text { MONTH } \\
\text { (Kgal) }\end{array}$ & $\begin{array}{r}\text { TOTAL } \\
\text { PUAPED } \\
\text { (Kgal) }\end{array}$ & $\begin{array}{l}\text { DRAIN- } \\
\text { ABLE } \\
\text { LICAUIO } \\
\text { REMAIN } \\
\text { (Kgal) }\end{array}$ & $\begin{array}{l}\text { PUAP- } \\
\text { ABLE } \\
\text { LIOUID } \\
\text { REMAIN } \\
\text { (Kgal) }\end{array}$ & $\begin{array}{l}\text { SLWDGE } \\
\text { (Kgat) }\end{array}$ & $\begin{array}{l}\text { SALTCAKE } \\
\text { (Kgal) }\end{array}$ & $\begin{array}{l}\text { LIOUIDS } \\
\text { VoLUnE } \\
\text { nethoo }\end{array}$ & $\begin{array}{l}\text { SOLIDS } \\
\text { VOLUAE } \\
\text { METHOD }\end{array}$ & $\begin{array}{l}\text { SOLIDS } \\
\text { VOLLNE } \\
\text { UPDATE }\end{array}$ & $\begin{array}{l}\text { SOLIDS } \\
\text { UPDATE } \\
\text { SOURCE } \\
\text { SEE } \\
\text { FOOTMOTE }\end{array}$ & $\begin{array}{l}\text { LAST } \\
\text { PHOTO } \\
\text { DATE }\end{array}$ & $\begin{array}{l}\text { CHG } \\
\text { SINCE } \\
\text { LAST } \\
\text { MNTHLY } \\
\text { REPORT }\end{array}$ \\
\hline & & & & & & t+t+t+t & SX FARM & Status & t+t+t+t & & & & & & & & & \\
\hline $5 x-101$ & $D C$ & SOUND & IPI & 456 & 1 & 145 & 0.0 & 0.0 & 166 & 124 & 112 & 343 & $p$ & FP & $04 / 28 / 82$ & & $03 / 10 / 89$ & \\
\hline $5 x-102$ & DSSF & sound & /PI & 543 & 0 & 183 & 0.0 & 0.0 & 183 & 177 & 117 & 426 & $p$ & $\boldsymbol{n}$ & $04 / 28 / 82$ & & $01 / 07 / 88$ & \\
\hline $5 x-103$ & MCPLX & sound & /PI & 652 & 1 & 232 & 0.0 & 0.0 & 233 & 211 & 115 & 536 & F & $\mathbf{s}$ & $07 / 15 / 91$ & & $12 / 17 / 87$ & \\
\hline $5 x-104$ & DSSF & ASIM LKR & IPI & 614 & 0 & 201 & 0.0 & 113.2 & 201 & 195 & 136 & 478 & $F$ & $\mathbf{s}$ & $07 / 07 / 89$ & & $09 / 08 / 88$ & \\
\hline $5 x-105$ & DSSF & SOUnD & IPI & 683 & 0 & 261 & 0.0 & 0.0 & 261 & 238 & $\mathbf{7 3}$ & 610 & $\mathbf{P}$ & $\mathbf{F}$ & $04 / 28 / 82$ & & $06 / 15 / 88$ & \\
\hline $5 x-106$ & MCPLX & soumo & API & 538 & 61 & 194 & 0.0 & 0.0 & 255 & 233 & 12 & 465 & $\mathbf{F}$ & Ps & $10 / 28 / 80$ & & 06/01/89 & \\
\hline $5 x-107$ & MCPLX & ASIm LKR & IS/IP & 104 & 0 & 5 & 0.0 & 0.0 & 5 & 0 & 104 & 0 & $\mathbf{p}$ & $n$ & $04 / 28 / 82$ & & $03 / 06 / 87$ & \\
\hline $5 x-108$ & NCPLX & ASID LKR & IS/IP & 87 & 0 & 5 & 0.0 & 0.0 & 5 & 0 & 87 & 0 & $p$ & $\boldsymbol{M}$ & $12 / 31 / 93$ & & $03 / 06 / 87$ & (f) \\
\hline $5 x-109$ & MCPLX & ASID LXR & IS/IP & 250 & 0 & 10 & 0.0 & 0.0 & 10 & 0 & 0 & 250 & $P$ & $\boldsymbol{M}$ & $10 / 05 / 93$ & & $05 / 21 / 86$ & \\
\hline $5 x-110$ & NCPLX & ASID LXR & IS/IP & 62 & 0 & 0 & 0.0 & 0.0 & 0 & 0 & 62 & 0 & $\boldsymbol{M}$ & PS & $10 / 06 / 76$ & & $02 / 20 / 87$ & \\
\hline $5 x-111$ & ACPLX & ASED LKR & IS/IP & 125 & 0 & 7 & 0.0 & 0.0 & 7 & 0 & 125 & 0 & $M$ & PS & $05 / 31 / 74$ & & $03 / 10 / 87$ & \\
\hline $5 x-112$ & NCPLX & ASND LKR & IS/IP & 92 & 0 & 3 & 0.0 & 0.0 & 3 & 0 & 92 & 0 & $\mathbf{P}$ & $\boldsymbol{M}$ & $04 / 28 / 82$ & & $03 / 10 / 87$ & \\
\hline $5 x-113$ & NCPLX & ASMO LKR & IS/IP & 26 & 0 & 0 & 0.0 & 0.0 & 0 & 0 & 26 & 0 & $\mathbf{p}$ & $n$ & $04 / 28 / 82$ & & $03 / 18 / 88$ & \\
\hline $5 x-114$ & MCPLX & ASWD LKR & IS/IP & 181 & 0 & 14 & 0.0 & 0.0 & 14 & 0 & 181 & 0 & $p$ & n & $04 / 28 / 82$ & & $02 / 26 / 87$ & \\
\hline $5 x-115$ & MCPLX & ASmD LKR & IS/IP & 12 & 0 & 0 & 0.0 & 0.0 & 0 & 0 & 12 & 0 & $P$ & $n$ & $04 / 28 / 82$ & & $03 / 31 / 88$ & \\
\hline 15 sime & IGLE-S & TAMKS & TOTALS: & 4425 & 63 & 1260 & 0.0 & 113.2 & 1323 & 1178 & 1256 & 3108 & & & & & & \\
\hline
\end{tabular}


tABLE E-5. INVENTORY AND STATUS BY tANK

SINGLE-SHELL TAMKS

Decenber 31, 1993

\begin{tabular}{|c|c|c|c|c|c|c|c|c|c|c|c|c|c|c|c|c|c|c|c|}
\hline \multirow[b]{2}{*}{ TAnK } & \multicolumn{2}{|c|}{ tank status } & \multicolumn{8}{|c|}{ LIOUID VOLUME } & \multicolumn{2}{|c|}{ SOLIDS VOLUnE } & \multicolumn{5}{|c|}{ VOLLAE DETERAINATION } & \multirow[b]{2}{*}{$\begin{array}{l}\text { CHG } \\
\text { SINCE } \\
\text { LAST } \\
\text { MUTHLY } \\
\text { REPORT }\end{array}$} & \\
\hline & $\begin{array}{l}\text { MASTE } \\
\text { MATERIAL }\end{array}$ & $\begin{array}{l}\text { TAMK } \\
\text { INTEERITY }\end{array}$ & $\begin{array}{l}\text { STABIL/ } \\
\text { IsOLATION } \\
\text { STATUS }\end{array}$ & $\begin{array}{r}\text { TOTAL } \\
\text { maste } \\
\text { (xgal) }\end{array}$ & $\begin{array}{l}\text { SUPER- } \\
\text { MATANT } \\
\text { LIOUID } \\
\text { (Kgal) }\end{array}$ & $\begin{array}{l}\text { DRAIN- } \\
\text { ABLE } \\
\text { INTER- } \\
\text { STIT. } \\
\text { (Kgal) }\end{array}$ & $\begin{array}{l}\text { PUMPED } \\
\text { THIS } \\
\text { MOWTH } \\
\text { (Kgal) }\end{array}$ & $\begin{array}{l}\text { TOTAL } \\
\text { PUMPED } \\
\text { (Kgal) }\end{array}$ & $\begin{array}{l}\text { DRAIN- } \\
\text { ABLE } \\
\text { LIOUID } \\
\text { REMaIN } \\
\text { (Kgal) }\end{array}$ & $\begin{array}{l}\text { PUIPP- } \\
\text { ABLE } \\
\text { LIOUID } \\
\text { REMAIK } \\
\text { (Kgal) }\end{array}$ & $\begin{array}{l}\text { SLUDEE } \\
\text { (Kgal) }\end{array}$ & $\begin{array}{l}\text { SALTCAKE } \\
\text { (Kgal) }\end{array}$ & $\begin{array}{l}\text { lieuios } \\
\text { Volume } \\
\text { MeTKoD }\end{array}$ & $\begin{array}{l}\text { soldos } \\
\text { volune } \\
\text { metion }\end{array}$ & $\begin{array}{l}\text { SOLIDS } \\
\text { VOLUine } \\
\text { UPOATE }\end{array}$ & $\begin{array}{l}\text { SOLIDS } \\
\text { UPOATE } \\
\text { SOLRCE } \\
\text { SEE } \\
\text { FOOTMOTE }\end{array}$ & $\begin{array}{l}\text { LAST } \\
\text { PHOTO } \\
\text { DATE }\end{array}$ & & \\
\hline & & & & & & +4t+4t & T FARM S & status & ++t+t+ & & & & & & & & & & \\
\hline$T-101$ & $\operatorname{MCPLX}$ & ASNO LKR & IS/PI & 102 & 1 & 16 & 0.0 & 25.3 & 17 & $\mathbf{0}$ & 101 & 0 & $\mathbf{f}$ & $\mathbf{s}$ & $04 / 16 / 93$ & & $04 / 07 / 93$ & & \\
\hline$T-102$ & MCPLX & sound & IS/IP & 32 & 13 & $\mathbf{0}$ & 0.0 & 0.0 & 13 & 13 & 19 & $\mathbf{0}$ & $\mathbf{p}$ & FP & $00 / 31 / 84$ & & $06 / 28 / 89$ & & \\
\hline$T-103$ & $\operatorname{MCPLX}$ & ASNO LKR & IS/IP & 27 & 4 & $\mathbf{0}$ & 0.0 & 0.0 & 4 & $\mathbf{0}$ & 23 & $\mathbf{0}$ & $\mathbf{F}$ & FP & $11 / 29 / 83$ & (1) & $07 / 02 / 84$ & & $m$ \\
\hline$T-104$ & MCPLX & soun & $\mid P I$ & 445 & 3 & 47 & 0.0 & 0.0 & 50 & 44 & 442 & 0 & $\mathbf{P}$ & $m$ & $04 / 28 / 82$ & & $06 / 29 / 89$ & & \\
\hline$T-105$ & MCPLX & soum & IS/IP & 90 & 0 & 23 & 0.0 & 0.0 & 23 & 17 & 96 & O & P & $\mathbf{F}$ & $05 / 29 / 87$ & & $05 / 14 / 87$ & & \\
\hline$T-106$ & $\operatorname{MCPLX}$ & ASRD LKR & IS/IP & 21 & 2 & $\mathbf{0}$ & 0.0 & 0.0 & 2 & 0 & 19 & 0 & $\mathbf{P}$ & FP & $04 / 28 / 82$ & & $06 / 29 / 89$ & & \\
\hline$T-107$ & NCPLX & ASNO LKR & IPI & 180 & 9 & 13 & 0.0 & 0.0 & 22 & 16 & 171 & 0 & $\mathbf{P}$ & FP & $08 / 31 / 84$ & & $07 / 12 / 84$ & & in \\
\hline$T-108$ & MCPLX & ASND LKR & IS/IP & 44 & 0 & 0 & 0.0 & 0.0 & 0 & 0 & 44 & 0 & $\mathbf{P}$ & $n$ & $04 / 28 / 82$ & & $07 / 17 / 84$ & & \\
\hline$T-109$ & MCPLX & ASTO LKR & IS/IP & 58 & 0 & $\mathbf{0}$ & 0.0 & 0.0 & 0 & 0 & 58 & 0 & $\boldsymbol{n}$ & $\mathbf{m}$ & $12 / 30 / 84$ & (1) & $02 / 25 / 93$ & & \\
\hline$T-110$ & nCPLX & soumo & IPI & 379 & 3 & 39 & 0.0 & 0.0 & 42 & 36 & 376 & 0 & $P$ & FP & $04 / 28 / 82$ & & $07 / 12 / 84$ & & \\
\hline$T-111$ & $n \cos x$ & ASPD LKR & /PI & 458 & 2 & 69 & 0.0 & 0.0 & 51 & 45 & 456 & 0 & p & FP & $04 / 28 / 82$ & & $08 / 02 / 84$ & & \\
\hline$T-112$ & NCPLX & sourd & IS/IP & 67 & 7 & 0 & 0.0 & 0.0 & 7 & 7 & 60 & 0 & $\mathbf{P}$ & FP & $04 / 28 / 82$ & & $08 / 01 / 84$ & & \\
\hline$T-201$ & MCPLX & sound & IS/IP & 29 & 1 & 3 & 0.0 & 0.0 & 4 & 0 & 28 & 0 & $n$ & PS & $05 / 31 / 78$ & & $04 / 15 / 86$ & & \\
\hline$T-202$ & NCPLX & sound & IS/IP & 21 & 0 & 2 & 0.0 & 0.0 & 2 & 0 & 21 & 0 & FP & $P$ & $07 / 12 / 81$ & & $07 / 06 / 89$ & & \\
\hline$T-203$ & NCPLX & souvio & IS/IP & 35 & 0 & 4 & 0.0 & 0.0 & 4 & 0 & 35 & 0 & $n$ & PS & $01 / 31 / 78$ & & $08 / 03 / 89$ & & \\
\hline$T-204$ & NCPLX & SCund & IS/IP & 38 & 0 & 4 & 0.0 & 0.0 & 4 & 0 & 38 & 0 & FP & $\mathbf{P}$ & $07 / 22 / 81$ & & $08 / 03 / 89$ & & \\
\hline $16 \sin$ & GLE-SHELL & TAMKS & TOTALS: & 2034 & 45 & 200 & 0.0 & 25.3 & 245 & 178 & 1989 & 0 & & & & & & & \\
\hline
\end{tabular}


TABLE E-5. INVENTORY AMD STATUS BY TAMK SINGLE-SHELL TAMKS

December 31, 1993

Tank STatus

\section{ram}

LIOUID VOLUne

DRAIN-

SUPER- ABLE

STABIL/ TOTAL MATANT IMTER- THIS TOTAL LIOUID LIOUID

MASTE TAMK ISOLATIOO UASTE LIOUID STIT. MONTH PUMPED REMAIN REMAIN Tami mitenial IMTECRity status (Koal) (Kgal) (Kgal) (Kgal) (Kgal) (Kgal) (Kgal)
SOLIDS VOLUTE

sol...............

VOLUME DETERHIMATION
SOLIOS
UPDATE

\begin{tabular}{|c|c|c|c|c|c|c|c|c|c|c|c|c|c|c|c|c|}
\hline & & & & & ++ & FARM & status & t+t+t+t & & & & & & & & \\
\hline Tx-101 ncetx & socind & IS/IP & 87 & 3 & 2 & 0.0 & 0.0 & 5 & 0 & 84 & 0 & $F$ & $\mathbf{P}$ & $02 / 02 / 84$ & (1) & $10 / 24 / 85$ \\
\hline$T x-102 \operatorname{mcplx}$ & soun & IS/IP & 217 & 0 & 22 & 0.0 & 94.4 & 22 & 0 & $\mathbf{0}$ & 217 & $\mathbf{n}$ & $\mathbf{s}$ & $08 / 31 / 86$ & & $10 / 31 / 85$ \\
\hline Tx-103 meptx & sound & IS/IP & 157 & 0 & 15 & 0.0 & 68.3 & 15 & 0 & 157 & O & $\mathbf{F}$ & $\mathbf{s}$ & $08 / 14 / 80$ & & $10 / 31 / 85$ \\
\hline$T x-104$ rcpex & soum & IS/IP & 65 & 1 & 14 & 0.0 & 3.6 & 15 & 0 & 0 & 64 & $\mathbf{f}$ & FP & $04 / 06 / 84$ & & $10 / 16 / 84$ \\
\hline$T x-105$ घCPLX & ASE LKR & IS/IP & 609 & 0 & 20 & 0.0 & 121.5 & 20 & 0 & 0 & 609 & $\boldsymbol{n}$ & PS & $08 / 22 / 77$ & & $10 / 26 / 89$ \\
\hline TX-106 NCPLXX & soum & IS/IP & 453 & 0 & 10 & 0.0 & 134.6 & 10 & 0 & 0 & 453 & $\boldsymbol{n}$ & $\mathbf{s}$ & $08 / 29 / 77$ & & $10 / 31 / 85$ \\
\hline TX-107 MCPLX & ASTD LKR & IS/IP & 36 & 1 & 1 & 0.0 & 0.0 & 2 & 0 & 0 & 35 & FP & FP & $01 / 20 / 84$ & (1) & $10 / 31 / 85$ \\
\hline$T x-108$ meptx & sound & IS/IP & 134 & 0 & 0 & 0.0 & 13.7 & 0 & 0 & 0 & 134 & $\mathbf{P}$ & FP & $05 / 30 / 83$ & & $09 / 12 / 89$ \\
\hline $1 x-109$ meplx & soun & IS/IP & 384 & 0 & 10 & 0.0 & $\mathbf{2} .3$ & 10 & 0 & 0 & 304 & $\mathbf{f}$ & PS & $05 / 30 / 83$ & & $10 / 24 / 89$ \\
\hline$T x-110$ mCPLX & ASED LKR & IS/IP & 462 & 0 & 15 & 0.0 & 115.1 & 15 & 0 & o & 462 & $n$ & PS & $05 / 30 / 83$ & & $10 / 24 / 89$ \\
\hline TX-111 & sown & IS/IP & 370 & 0 & 9 & 0.0 & 98.4 & 9 & 0 & 0 & 370 & $n$ & PS & $07 / 26 / 77$ & & $09 / 12 / 89$ \\
\hline$T x-112$ MCPLX & soumo & IS/IP & 649 & 0 & 24 & 0.0 & 94.0 & 24 & 0 & 0 & 649 & $\mathbf{p}$ & PS & $05 / 30 / 83$ & & $11 / 19 / 87$ \\
\hline$T x-113$ MCPLX & ASTD LKR & IS/IP & 607 & 0 & 16 & 0.0 & 19.2 & 16 & 0 & 0 & 607 & $n$ & PS & $05 / 30 / 83$ & & $04 / 11 / 83$ \\
\hline$T x-114$ NCPLX & ASND LKR & IS/IP & 535 & 0 & 15 & 0.0 & 104.3 & 15 & 0 & o & 535 & $\boldsymbol{n}$ & PS & $05 / 30 / 83$ & & $04 / 11 / 83$ \\
\hline$T x-115$ NCPLX & ASTD LKR & IS/IP & 640 & $\mathbf{0}$ & 19 & 0.0 & 99.1 & 19 & 0 & 0 & 640 & $n$ & $\mathbf{s}$ & $03 / 25 / 83$ & & $06 / 15 / 80$ \\
\hline$T x-116$ NCPLX & ASND LKR & $15 / 1 P$ & 631 & 0 & 23 & 0.0 & 23.8 & 23 & 0 & o & 631 & $\boldsymbol{M}$ & PS & $03 / 31 / 72$ & & $10 / 17 / 89$ \\
\hline $7 x-117$ MCPLX & ASHD LKR & IS/IP & 626 & 0 & 8 & 0.0 & 54.3 & 8 & 0 & o & 626 & M & PS & $12 / 31 / 71$ & & $04 / 11 / 83$ \\
\hline TX-118 NCPLX & soum & IS/IP & 347 & $\mathbf{0}$ & 27 & 0.0 & 89.1 & 27 & 0 & o & 347 & $\mathbf{f}$ & $\mathbf{s}$ & $11 / 17 / 80$ & & $12 / 19 / 79$ \\
\hline 18 SINGLE-SMEL & TAMKS & TOTALS: & 7009 & 5 & 250 & 0.0 & 1205.7 & 255 & 0 & 241 & 6763 & & & & & \\
\hline
\end{tabular}


TABLE E-5. INVENTORY AND STATUS BY TANK SIMGLE-SHELL TAMKS December 31, 1993

\begin{tabular}{|c|c|c|c|c|c|c|c|c|c|c|c|c|c|c|c|c|c|c|}
\hline & TAMIK SI & TATUS & & & & LIOUI & VOLLARE & & & & SOLIDS & Volume & & VOL & E DETER & IMATIOW & & \\
\hline MYK & $\begin{array}{l}\text { MASTE } \\
\text { MATERIAL }\end{array}$ & $\begin{array}{l}\text { TAMK } \\
\text { INTECRITY }\end{array}$ & $\begin{array}{l}\text { STABILI } \\
\text { ISOLATIOH } \\
\text { STATUS }\end{array}$ & $\begin{array}{l}\text { TOTAL } \\
\text { MASTE } \\
\text { (Kgal) }\end{array}$ & $\begin{array}{l}\text { SUPER- } \\
\text { MATAMT } \\
\text { LIOUID } \\
\text { (Kogl) }\end{array}$ & $\begin{array}{l}\text { DRAIN- } \\
\text { ABLE } \\
\text { IMTER- } \\
\text { STIT. } \\
\text { (Kgal) }\end{array}$ & $\begin{array}{c}\text { PUMPED } \\
\text { THIS } \\
\text { MONTK } \\
\text { (Kgal) }\end{array}$ & $\begin{array}{l}\text { TOTAL } \\
\text { PUMPED } \\
\text { (Kgal) }\end{array}$ & $\begin{array}{l}\text { DRAIN- } \\
\text { ABLE } \\
\text { LICUID } \\
\text { REMIN } \\
\text { (Keal) }\end{array}$ & $\begin{array}{l}\text { PUAP- } \\
\text { ABLE } \\
\text { LIOUID } \\
\text { REAMIN } \\
\text { (Keal) }\end{array}$ & $\begin{array}{l}\text { SLLDEE } \\
\text { (Kgel) }\end{array}$ & $\begin{array}{l}\text { SALTCAKE } \\
\text { (KGal) }\end{array}$ & $\begin{array}{l}\text { LIOUIDS } \\
\text { Volune } \\
\text { METHOO }\end{array}$ & $\begin{array}{l}\text { SOLIDS } \\
\text { VOLUIE } \\
\text { METHCo }\end{array}$ & $\begin{array}{l}\text { SOLIDS } \\
\text { VOLUAE } \\
\text { UPOATE }\end{array}$ & $\begin{array}{l}\text { SOL IOS } \\
\text { UPDATE } \\
\text { SOURCE } \\
\text { SEE } \\
\text { FOOTWOTE }\end{array}$ & $\begin{array}{l}\text { LAST } \\
\text { PHOTO } \\
\text { DATE }\end{array}$ & $\begin{array}{l}\text { CHG } \\
\text { SINCE } \\
\text { LAST } \\
\text { MUTHLY } \\
\text { REPORT }\end{array}$ \\
\hline
\end{tabular}

\begin{tabular}{|c|c|c|c|c|c|c|c|c|c|c|c|c|c|c|c|c|c|}
\hline & & & & & & ++1 & FARM S & tatus & +4 & & & & & & & & \\
\hline$T Y-10: 1$ & $\operatorname{mcPlx}$ & ASN LKR & IS/IP & 118 & 0 & 0 & 0.0 & 8.2 & 0 & 0 & 118 & 0 & $P$ & $\mathbf{F}$ & $04 / 28 / 82$ & & $08 / 22 / 89$ \\
\hline $\mid \gamma-102 I$ & MCPLX & sound & IS/IP & 64 & 0 & 14 & 0.0 & 6.6 & 16 & 0 & 0 & 64 & $\mathbf{P}$ & FP & $06 / 28 / 82$ & & $07 / 07 / 87$ \\
\hline$\pi-103$ & MCPLX & ASHD LKR & IS/IP & 162 & 0 & 5 & 0.0 & 11.5 & 5 & $\mathbf{0}$ & $: 62$ & 0 & $\mathbf{P}$ & FP & 07/09/82 & & $08 / 22 / 89$ \\
\hline$\pi-104$ & MCPLX & ASTD LKR & IS/IP & 46 & 3 & 12 & 0.0 & 0.0 & 15 & 0 & 43 & 0 & $\mathbf{P}$ & FP & $06 / 27 / 90$ & (1) & $11 / 03 / 87$ \\
\hline$T Y-105$ & MCPLX & ASID LKR & IS/IP & 231 & 0 & 0 & 0.0 & 3.6 & 0 & 0 & 231 & $\bullet$ & $\mathbf{P}$ & $n$ & $04 / 28 / 82$ & & $09 / 07 / 89$ \\
\hline$T \gamma-106$ & MCPLX & ASND LKR & IS/IP & 17 & 0 & 0 & 0.0 & 0.0 & 0 & 0 & 17 & 0 & $\mathbf{P}$ & $n$ & $04 / 28 / 82$ & & $08 / 22 / 89$ \\
\hline 6 SIMGL & LE-SHELL & TANKS & TOTALS: & 638 & 3 & 31 & 0.0 & 29.9 & 36 & 0 & 571 & 64 & & & & & \\
\hline & & & & & & +++1 & FARM SI & ATUS & $t+4$ & & & & & & & & \\
\hline U-101 & MCPLX & ASHD LKR & $15 / 1 P$ & 25 & 3 & 0 & 0.0 & 0.0 & 3 & $\mathbf{0}$ & 22 & o & $\mathbf{P}$ & m & $04 / 28 / 82$ & & $06 / 19 / 79$ \\
\hline U-102 & MCPLX & sound & IPI & 374 & 18 & 126 & 0.0 & 0.0 & 144 & 122 & 43 & 313 & $\mathbf{P}$ & $m$ & $04 / 28 / 82$ & & $06 / 08 / 89$ \\
\hline$U-103$ & MCPLX & sound & IPI & 468 & 13 & 176 & 0.0 & 0.0 & 189 & 166 & 32 & 423 & $\mathbf{p}$ & FP & $04 / 28 / 82$ & & $09 / 13 / 88$ \\
\hline$U-104$ & MCPLX & ASWD LKR & IS/IP & 122 & 0 & 7 & 0.0 & 0.0 & 7 & 0 & 122 & o & $\mathbf{P}$ & mp & $04 / 28 / 82$ & & $08 / 10 / 89$ \\
\hline$U-105$ & MCPLX & sourd & IPI & 418 & 37 & 142 & 0.0 & 0.0 & 179 & 157 & 32 & 349 & $\mathbf{F n}$ & PS & $09 / 30 / 78$ & & $07 / 07 / 88$ \\
\hline$U-106$ & MCPLX & soum & /PI & 226 & 15 & 68 & 0.0 & 0.0 & 83 & 61 & 26 & 185 & $F$ & PS & $12 / 30 / 83$ & & $07 / 07 / 88$ \\
\hline$U-107$ & DSSF & soum & /PI & 406 & 31 & 147 & 0.0 & 0.0 & 178 & 156 & 15 & $36 C$ & $\mathbf{F}$ & $\mathbf{s}$ & $12 / 30 / 83$ & & $10 / 27 / 80$ \\
\hline$U-108$ & MCPLX & SOUND & /PI & 468 & 24 & 172 & 0.0 & 0.0 & 196 & 174 & 29 & 415 & $\mathbf{F}$ & s & $12 / 30 / 83$ & & $09 / 12 / 84$ \\
\hline$U-109$ & MCPLX & sound & $\mid \mathrm{PI}$ & 463 & 19 & 163 & 0.0 & 0.0 & 182 & 160 & 48 & 396 & F & $\mathbf{F}$ & $11 / 13 / 77$ & & $07 / 07 / 88$ \\
\hline$U-110$ & MCPLX & ASYD LKR & IS/PI & 186 & 0 & 15 & 0.0 & 0.0 & 15 & 9 & 186 & o & $n$ & $\mathbf{M}$ & $12 / 30 / 84$ & (1) & $12 / 11 / 84$ \\
\hline U-111 & DSSF & sound & $|P|$ & 329 & 0 & 122 & 0.0 & 0.0 & 122 & 99 & 26 & 303 & PS & FPS & $04 / 28 / 82$ & & $06 / 23 / 88$ \\
\hline U-112 & NCPLX & ASYD LKR & IS/IP & 49 & 4 & 0 & 0.0 & 0.0 & 4 & 0 & 45 & 0 & $\mathbf{P}$ & MP & $02 / 10 / 84$ & (1) & 08/03/89 \\
\hline
\end{tabular}


TABLE E-5. INVENTORY AMD STATUS BY TAMK

SIMGLE-SHELL TAMKS

Deceuber 31, 1993

\begin{tabular}{|c|c|c|c|c|c|c|c|c|c|c|c|c|c|c|c|c|c|c|}
\hline & TANK ST & Tatus & & & & LIOUID & VOLLAE & & & & solios $v$ & Volune & & roll & ME DETERMI & INATION & & \\
\hline TAMK & $\begin{array}{l}\text { MSTE } \\
\text { matenIal }\end{array}$ & $\begin{array}{l}\text { TAMK } \\
\text { InTEeaITY }\end{array}$ & $\begin{array}{l}\text { STABIL/ } \\
\text { ISOLATION } \\
\text { STATUS }\end{array}$ & $\begin{array}{l}\text { Total } \\
\text { unste } \\
\text { (Kgal) }\end{array}$ & $\begin{array}{l}\text { SUPER- } \\
\text { MATAMT } \\
\text { LICUID } \\
\text { (Kgal) }\end{array}$ & $\begin{array}{l}\text { DRAIN- } \\
\text { ABLE } \\
\text { INTER- } \\
\text { STIT. } \\
\text { (Keal) }\end{array}$ & $\begin{array}{l}\text { PUImED } \\
\text { THIS } \\
\text { Mowin } \\
\text { (Kgal) }\end{array}$ & $\begin{array}{l}\text { TOTAL } \\
\text { PUMPED } \\
\text { (Keal) }\end{array}$ & $\begin{array}{l}\text { DRAIR- } \\
\text { ABLE } \\
\text { LICUID } \\
\text { REMAII } \\
\text { (Real) }\end{array}$ & $\begin{array}{l}\text { PUIP. } \\
\text { ABLE } \\
\text { LIOUID } \\
\text { REMII } \\
\text { (KoSI) }\end{array}$ & $\begin{array}{l}\text { SLLDEE } \\
\text { (Kegl) }\end{array}$ & $\begin{array}{l}\text { SALTCAKE } \\
\text { (Kgal) }\end{array}$ & 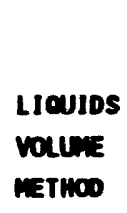 & $\begin{array}{l}\text { solibs } \\
\text { volume } \\
\text { netrice }\end{array}$ & $\begin{array}{l}\text { solios } \\
\text { volume } \\
\text { uponte }\end{array}$ & $\begin{array}{l}\text { SOLIOS } \\
\text { UPDATE } \\
\text { SOURCE } \\
\text { SEE } \\
\text { FOOTWOTE }\end{array}$ & $\begin{array}{l}\text { LAST } \\
\text { PHOTO } \\
\text { DATE }\end{array}$ & $\begin{array}{l}\text { CHG } \\
\text { SINCE } \\
\text { LAST } \\
\text { MWTHLY } \\
\text { REPORT }\end{array}$ \\
\hline U-201 & $\operatorname{mcp} 2 x$ & sound & $15 / 1 P$ & 5 & 1 & 0 & 0.0 & 0.0 & 1 & 0 & 4 & 0 & M & $\mathbf{s}$ & $08 / 15 / 79$ & & $08 / 03 / 89$ & \\
\hline v-202 & $\operatorname{mcplx}$ & soun & $15 / 1 P$ & 5 & 1 & 0 & 0.0 & 0.0 & 1 & 0 & 4 & o & $\boldsymbol{n}$ & $\mathbf{s}$ & $08 / 15 / 79$ & & $08 / 08 / 89$ & \\
\hline$U-203$ & veptx & sound & $15 / 1 P$ & 3 & 1 & 0 & 0.0 & 0.0 & 1 & 0 & 2 & 0 & n & $\mathbf{s}$ & $08 / 15 / 79$ & & $06 / 13 / 89$ & \\
\hline v-204 & $\operatorname{rcsex}$ & soum & IS/IP & 3 & 1 & 0 & 0.0 & 0.0 & 1 & 0 & 2 & o & n & $\mathbf{s}$ & $00 / 15 / 79$ & & $06 / 13 / 89$ & \\
\hline $16 \mathrm{stm}$ & IEL-SMELL & TAMKs & Totals: & 3550 & 168 & 1138 & 0.0 & 0.0 & 1306 & 1104 & 638 & 2744 & & & & & & \\
\hline easoso & TOTAL & & & 36009 & 568 & 6273 & 28 & $38 \pi$ & 6842 & 5372 & 12180 & 23345 & & & & & & \\
\hline
\end{tabular}

Total waste is calculated so the sum of sludige and salteake plus supernate.

The catesory "Inter in Isoloted (II) mes changed to "intrusion Prevention" (IP) in June 1993. See section C. "Tank end Equipment Code and Status Definitions"

(1) IMC-SD-RE-TI-17B SST STABILIZATION RECOBD, Latest revision

(2) TAMK FARas SST ENGIMEER MOWTMLY IMPUT (Retained $10 \mathrm{gr}$ in Monthly Sumary Report euthor's office)

See next pege for footnotes in "Chg Since Last Monthly Report" 
TABLE E-5. IGVENTORY ANO STATUS OY tAMK

SIMGLE-SHELL TAMKS

Deceuber 31, 1993

\begin{tabular}{|c|c|c|c|c|c|c|c|c|c|c|c|c|c|c|c|c|c|c|}
\hline & TANK ST & TATUS & & & & LIOUI0 & Volune & & & & EOLIDS & rouere & & Voll & EE DETERA & Imarion & & \\
\hline Tanx & $\begin{array}{l}\text { MSTE } \\
\text { MATERIAL }\end{array}$ & $\begin{array}{l}\text { TamK } \\
\text { IntegaItr }\end{array}$ & $\begin{array}{l}\text { STABIL/ } \\
\text { Isolation } \\
\text { Status }\end{array}$ & $\begin{array}{c}\text { TOTAL } \\
\text { maste } \\
\text { (Kgal) }\end{array}$ & $\begin{array}{l}\text { SUPER- } \\
\text { MATAMT } \\
\text { LICUID } \\
\text { (Kgal) }\end{array}$ & $\begin{array}{l}\text { DRAIM- } \\
\text { ABLE } \\
\text { IWTER- } \\
\text { STIT. } \\
\text { (Kgal) }\end{array}$ & $\begin{array}{l}\text { PLipED } \\
\text { THIS } \\
\text { MouTh } \\
\text { (Kgal) }\end{array}$ & $\begin{array}{l}\text { TOTAL } \\
\text { PUPED } \\
\text { (Keal) }\end{array}$ & $\begin{array}{l}\text { DRAIN- } \\
\text { ABLE } \\
\text { LICUID } \\
\text { REMaIN } \\
\text { (Keal) }\end{array}$ & $\begin{array}{l}\text { PISP- } \\
\text { NoLE } \\
\text { LICUID } \\
\text { RemaIn } \\
\text { (Keal) }\end{array}$ & $\begin{array}{l}\text { SLDEE } \\
\text { (Keal) }\end{array}$ & $\begin{array}{l}\text { saltcuke } \\
\text { (Keal) }\end{array}$ & $\begin{array}{l}\text { LIovids } \\
\text { voulume } \\
\text { metros }\end{array}$ & $\begin{array}{l}\text { SOLIDS } \\
\text { VOLLINE } \\
\text { netHod }\end{array}$ & $\begin{array}{l}\text { SOLIDS } \\
\text { VOCunE } \\
\text { UPoATE }\end{array}$ & $\begin{array}{c}\text { Sollos } \\
\text { upoATE } \\
\text { Sounce } \\
\text { SEE } \\
\text { Footmote }\end{array}$ & $\begin{array}{l}\text { LAST } \\
\text { PHOTO } \\
\text { DATE }\end{array}$ & $\begin{array}{l}\text { CHG } \\
\text { SINCE } \\
\text { LAST } \\
\text { MUTMLY } \\
\text { REPORT }\end{array}$ \\
\hline
\end{tabular}

FOOTMOTES:

(a) ax-110 - Following information fram single-shell Tanks cosenizent Enpineer

Puping beoen December 4, 1993.

Total mate (Supernatent + Solids) - $199.0 \mathrm{kgol}$

supernatent - $0 \mathrm{Kgel}$

praineble Interatitial - $15.4 \mathrm{kgal}$

I Puped this Month $-4.0 \mathrm{kgal}$

Total Puped $-4.0 \mathrm{kgal}$

Drainable Liquid Remining - $16.6 \mathrm{kgal}$

Pupeble Liquid Remaining $-6.0 \mathrm{Kael}$

studee - $189.0 \mathrm{kal}$

sal tcake - $9.0 \mathrm{rgal}$

(b) a-111 - Following informtion from single-shell Tenks comizant Engineer

puping begen october 22, 1993

Total meste (supernatent + Solids) - $211.0 \mathrm{kgal}$

Supermatent - O Keal

Drainmble Interstitial - $14.5 \mathrm{Kgal}$

Puped this Month - $23.7 \mathrm{Kgal}$

Total Puped - $54.7 \mathrm{Koal}$

Drainable Liquid Remining - $14.5 \mathrm{Kgal}$

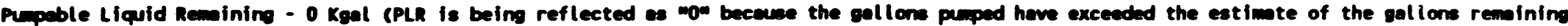
then puping begen. PLR will be re-eatimated uhen puping is completed.)

Sludoe - $68.0 \mathrm{Kgal}$

Sal tcake - $143.0 \mathrm{Kgal}$ 
TABLE E-5. IMVENTORY AMD STATUS BY tAMK SIMGLE-SHELL TANKS

Decenber 31, 1993

TAMK STATUS

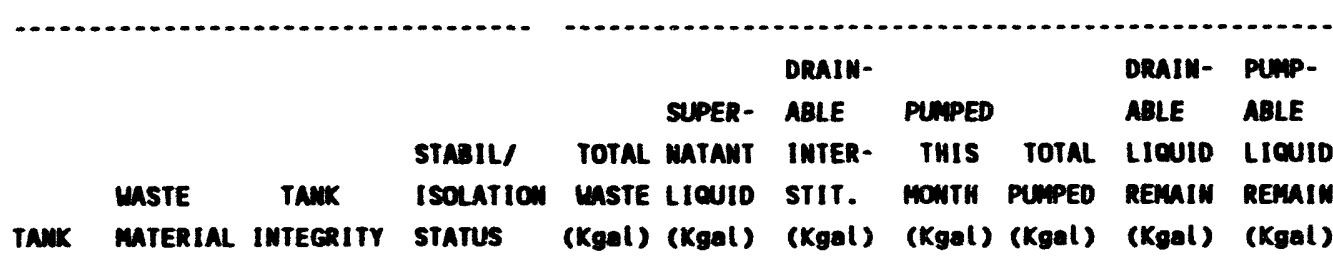

solids volune

SLlOGE SALTCAKE
(Kgal) (Kgal)

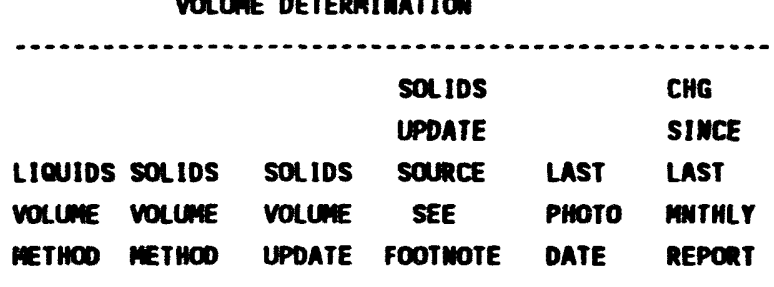

An imvestigation was conducted and an evaluation completed in Decenber 1993, concerning discrepencies between current weste inventory and manual level readings. The review of past waste level surveillence records and in-tank photos revealed the reasons for these discrepencies. Mary tenks have erroneous fic level readings due to mounds and valleys on the waste surface, holes in the waste surface where the FIC falls, and/or debris below the tape plumet which causes a discrepancy between current values and the waste level surveillence records. Also, several fic tapes are set in the intrusion mode, wich will shou a sall discrepency.

I. The following five tanks were changed to reflect the results of this imvestigation:

(c) 5-106

Total maste (Supernatent + Solids) $-479.0 \mathrm{Kgal}$

supernatant - $4 \mathrm{Kgal}$

Draineble Interstitial - $186 \mathrm{Kgal}$

Puped this Month - M/A Kgal

Total Puped - $97 \mathrm{Kgal}$

Draineble Liquid Remaining - $190 \mathrm{Kgal}$

Pupable Liquid Remaining - $168 \mathrm{kgal}$

sludge - $28.0 \mathrm{Kgal}$

Sal tcake - $447.0 \mathrm{Kgal}$

It appears the solids leve! was not updoted after the sal twell puiping campaign in July 1979, when 97,000 gal of liquid was punped. Pumplng is to resume

in FY 1996. This tank has had numerous basel ine adjustments for unexplainable rising liquid levels. 
TABLE E-5. IMVEMTORY AMO STATUS BY TAMK

SIMGLE-SHELL TAMKS

Decenber 31, 1993

TAm STATUS

maste tank

TAM MATERIAL IMTEERITY

\section{STABIL/}

isoutio

S-107

Total mate (supernatent + solfds) - $376.0 \mathrm{Kgel}$

supernatent - $14 \mathrm{Kgal}$

Drainable Interstitial - $45 \mathrm{keal}$

Puped this Month - M/A Koal

Total Puped - W/A Koal

Drainable Liquid Remining - $59 \mathrm{Kgal}$

Pupeble Llquid Remining - $52 \mathrm{kgal}$

sludge - $293 \mathrm{kgal}$

Salteake $-69.0 \mathrm{kgal}$

Comparison of 1903 and 1907 photo packeges indicate an increase. Based on this influx an elevatian wes extrepolated for 1993, wich correlates uith the current liquid level. Draineble liquid and supernatent were chenged to reflect this increase.

This tenk is sound, Partial Interin isoleted, and not yet stebillized.

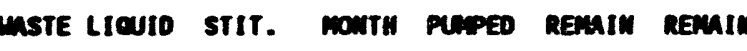

solios volure

\section{voluke}

DRAIN- PUMP-

SUPER- ABLE PUIPEd ABLE ABLE

TOTAL MATAMT INTER- TMIS TOTAL LICUID LICUID

(Kgal) (Kgal) (Kgal) (Kgal) (Kgal) (Kgal) (Kgal)

som von

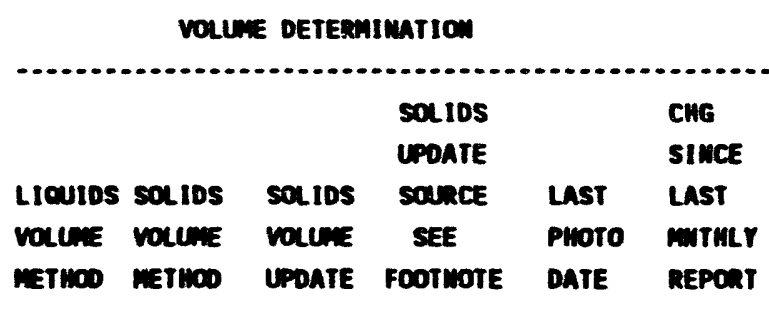

SLDeE SALTCAKE

(Keal) (Koel) 
TABLE E-5. ImVETOAY ANO STATUS BY tAMK

SIMGLE-SHELL TAMKS

Decenber 31, 1993

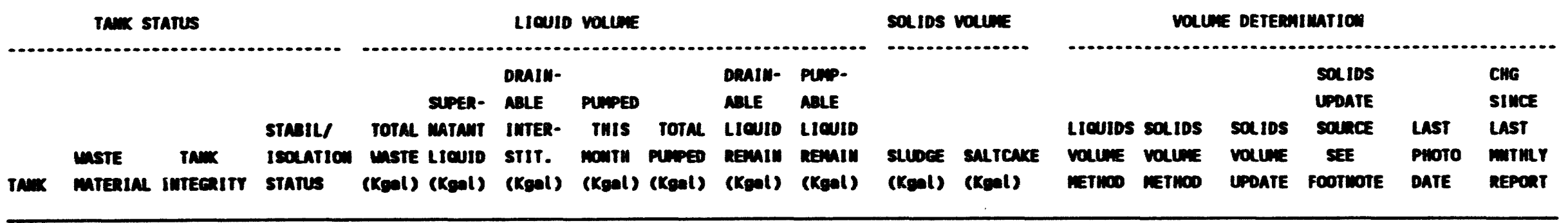

(e) $5-112$

Total mate (supernatent + solids) - $523.0 \mathrm{kgal}$

supernatent - O Keal

Drainble Interetitial - $110 \mathrm{kgal}$

punped this Month - w/A Kgal

I Totel puped - $125.1 \mathrm{kgal}$

Draindele Liquid Remining - $110 \mathrm{Kgal}$

Prepble Liquid Reminin - $107 \mathrm{kgal}$

studie $-4.9 \mathrm{kgal}$

Sel teake - $517.7 \mathrm{kgal}$

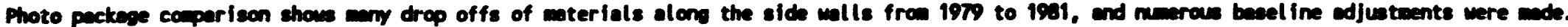
The level hes remined constent since 1961 and the 1987 photos are very siallar to the 1901 photce.

This tenk is Sourd, Partial Interin Isolated, and not yet stabllized. 
TABLE E-5. IMVENTORY ANO STATUS BY TAMK

SIWGLE-SHELL TAMKS

Deceuber 31, 1993

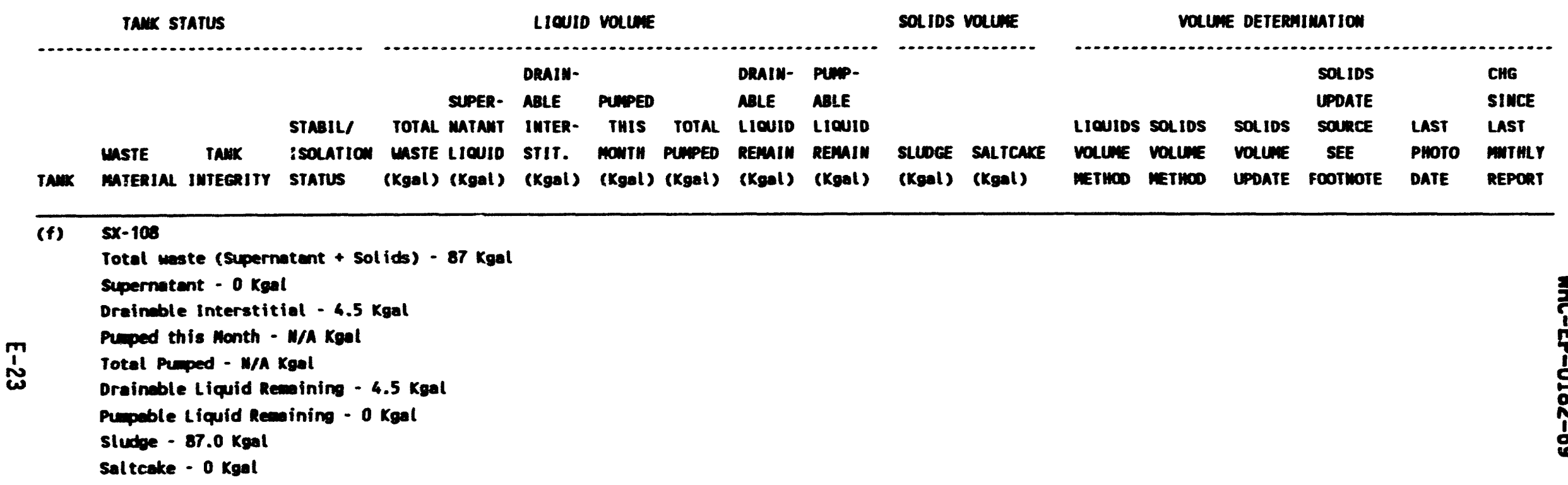

An incorrect length tape was installed as a replacement in October 1976. This tope, short by approximately 10 inches, was used to Adainistratively stabilize the tank in 1979. The correct length tape wes installed in danuary 1993, but the Monthly sumery Report was not updated with the correct voluse of waste.

This tank is an Assumed Leaker, Stabilized, and Intrusion Prevention completed. 
TABLE E-5. IMVEMTORY ANO STATUS BY tAMK

SIWGLE-SMELL TAMKS

December 31, 1993

\begin{tabular}{|c|c|c|c|c|c|c|c|c|c|c|c|c|c|c|c|c|c|c|}
\hline & tanx 5 & tatus & & & & Lioulo & volume & & & & solios & rocene & & vole & E DETERA & mation & & \\
\hline Tanx & $\begin{array}{l}\text { IASTE } \\
\text { matenINL }\end{array}$ & $\begin{array}{l}\text { Tamr } \\
\text { InTEeaITY }\end{array}$ & $\begin{array}{l}\text { stall } \\
\text { Isolation } \\
\text { stans }\end{array}$ & $\begin{array}{l}\text { Total } \\
\text { maste } \\
\text { (Keal) }\end{array}$ & $\begin{array}{l}\text { SUPER- } \\
\text { MATAMT } \\
\text { LICUID } \\
\text { (Kgel) }\end{array}$ & $\begin{array}{l}\text { DRAIN- } \\
\text { Aate } \\
\text { IWTER- } \\
\text { STIT. } \\
\text { (Kgal) }\end{array}$ & $\begin{array}{l}\text { PUIPED } \\
\text { TUIS } \\
\text { MOWTy } \\
\text { (Kol) }\end{array}$ & $\begin{array}{l}\text { TOTAL } \\
\text { Punped } \\
\text { (Keal) }\end{array}$ & $\begin{array}{l}\text { DRAin- } \\
\text { ABLE } \\
\text { LINulo } \\
\text { RExain } \\
\text { (Keal) }\end{array}$ & $\begin{array}{l}\text { PUPP- } \\
\text { ABLE } \\
\text { LIEUID } \\
\text { Remain } \\
\text { (Keal) }\end{array}$ & $\begin{array}{l}\text { stwoce } \\
\text { (Keol) }\end{array}$ & $\begin{array}{l}\text { SALtcake } \\
\text { (Keal) }\end{array}$ & $\begin{array}{l}\text { Licuios } \\
\text { volure } \\
\text { retime }\end{array}$ & $\begin{array}{l}\text { solids } \\
\text { volure } \\
\text { netwos }\end{array}$ & $\begin{array}{l}\text { Solios } \\
\text { vouUE } \\
\text { upoate }\end{array}$ & $\begin{array}{l}\text { solios } \\
\text { upoate } \\
\text { solnce } \\
\text { seE } \\
\text { footmote }\end{array}$ & $\begin{array}{l}\text { LAST } \\
\text { Photo } \\
\text { DATE }\end{array}$ & $\begin{array}{l}\text { CHE } \\
\text { SINCE } \\
\text { LAST } \\
\text { nimTY } \\
\text { REPORT }\end{array}$ \\
\hline
\end{tabular}

(s) $\quad T x-102$

Total mate (supernatent + Sol fds) - $217 \mathrm{kgol}$

aparnatent - 0 Keal

Drainble Interstitial - $22.0 \mathrm{kgal}$

i puped this Wonth - W/A Keal

1 Total muped - $94 \mathrm{kgal}$

Draineble Liquid Remining - $22.0 \mathrm{Kgal}$

Pupable Liquid Remining - 0 kgal

sludoe - 0 Kegl

Sel teake - $217 \mathrm{kgal}$

The incorrect length of tepe was used to measure surfece level in the tank ot the tile of stebilizotion in March ises. The feulty tepe wes repleced In Movenber 19a3, but the Monthly sumery Report was not updated with the correct volum.

This tank is an hasumed Leaker. Stabllized, and Intrusion Prevention completed. 
WHC-EP-0182-69

\section{APPENDIX F \\ PERFORMANCE SUMMARY}

F-1 
WHC-EP-0182-69

This page intentionally left blank.

F-2 
TABLE F-1. PERFORMANCE SUMMARY (Sheet 1 of 3)

WASTE VOLUMES (Kgallons)

December 31, 1993

INCREASES/DECREASES IN WASTE VOLUMES STORED IN DOUBLE-SHELL TANKS

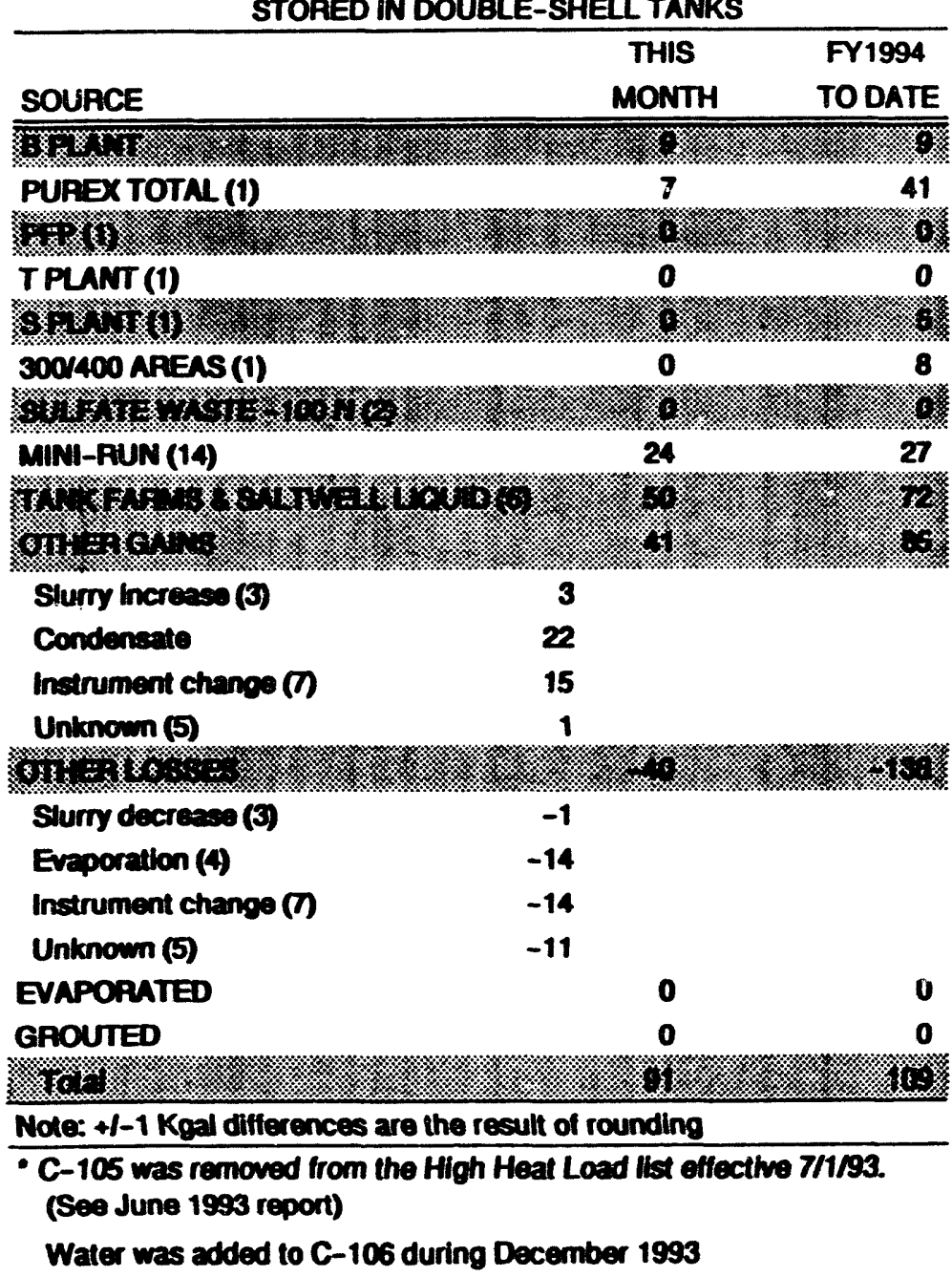

INCREASESTOECREASES IN WASTE VOLUMES STOAED IN SINGLE-SHELL TANKS

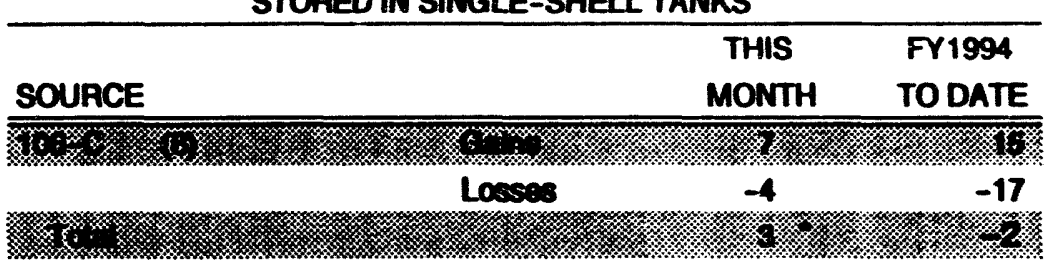

CUMULATIVE EVAPORATION - 1950 TO PRESENT WASTE VOLUME REDUCTION

\begin{tabular}{|c|c|}
\hline \multicolumn{2}{|l|}{ FACILTY } \\
\hline 242-B EVAPOANTOR(S) & 7172 \\
\hline 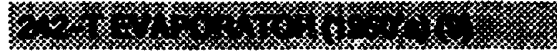 & 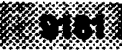 \\
\hline IN-TANK SOLDIFCATION UNT 1 (10) & 11876 \\
\hline IN-TAKK SOLDIFCATION UMT 2 (10) & 15295 \\
\hline 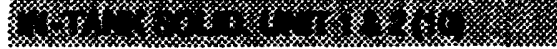 & 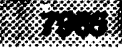 \\
\hline \multicolumn{2}{|c|}{ (after comersion of Unit 1 to a cooler for Unit 2) } \\
\hline 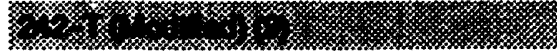 & 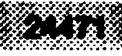 \\
\hline 242-S EVAPORATOR(11) & 41983 \\
\hline 242-A EVAPOAATOA (12) & 65227 \\
\hline 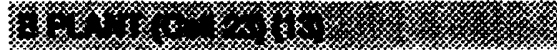 & 紧紧 \\
\hline PEDOX (12) & 12393 \\
\hline 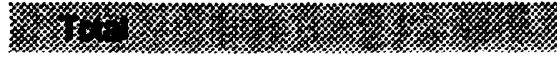 & 竖 \\
\hline \multicolumn{2}{|c|}{ TOTAL THAOUGHPUT } \\
\hline \multicolumn{2}{|l|}{ FACILITY } \\
\hline 242-A EVAPORATOA(12) & 182437 \\
\hline 242-S EVAPORATOR(11) & 134587 \\
\hline 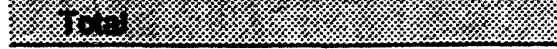 & 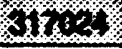 \\
\hline
\end{tabular}


MHC-EP-0182-69

TABLE F-1. PERFORMANCE SUMMARY

(Sheet 2 of 2)

\section{Footnotes:}

\section{IMCREASES/DFCREAGFS IIN MASTE YOLLMES}

(1) Including Flush

(2) Sulfate waste is generated from ion exchenge backfluahing and sand filter clean out, resulting in sulfate waste $\left(\mathrm{Man}_{2} \mathrm{SO}_{4}\right)$.

(3) Slurry increase/growth is caused by gas generation within the waste. The gas which is trapped in the waste expends in the tank caus ing the surface level and volume to increase. Slurry decrease results from the periodic release of gas in the uste.

(4) Aging maste tonks

(5) Unknown waste gaine or losses way be the reault of rounding calculations, cleen water slowly leaking throweh a valve, changes in levels (oxpentier/centrection) because of embient teiperature changes, different masuring devices beine uced by Tank form oparators, trenefore takino place during the end of the wonth. Tenk Farm ectivitice such es discel leneous water edditions not essocieted with facility maste onaration, or the addition of water mich is added to aging waste tenks and then eveporated off.

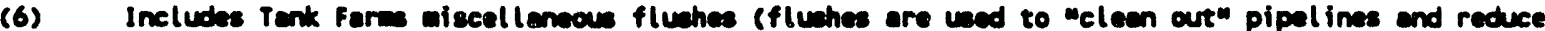
persomel expoeure, reduce potential for wate incempetibility, prevent line plugging, and reduce waste content of potential spills or leaks). and saltwell liquid, which reaults from puping of single-shell tenks to double-shall tenks.

(7) Liquid level masurcaent instrument chenose from the eutomtic fic to monul tepe (end vice versa) reault in unusul gains or losses becaue the monal tepe noy rest on en uneven crust surface giving - different reading frem that of the eutemetic FIC. These instrument changes are mode when the outantic FIC is out of service and the reading from the manul tepe is used for reporting purposes. The reported reading reverts back to the autcantic FIC then it is repaired.

(8) Water is pariodically added 106-C to provide eveporative cooling. Losese de to eveporation are calculated assuaing all losses are eveporative losses. som drniclls ore monitored weekly and same are enonitored every two weeks on tank 106-C. If there are $\mathrm{cmy}$ indicutions of a leak from this tank, the assuption that all losses are due to eveporation will be reevaluated.

\section{WASTE VOLUNE REDUCTION}

Currently inoperative. These eveporator syetem (242-1 and 242-T) were installed in 1952 in each of the two aperating areas to reavve water fram the waste, and ren for approximately 4 yr after which both unite were shut down. The 242-T Eveporator was reactiveted in December 1965, and shut down again in April 1976.

(10) Currently inoperative. These two in-tank solidification (ITS) units providad in-tank heating to promote in-tenk boiling or eveporation. The ITS Unit 1 etarted up March 1965, and ITS Unit 2 sterted up february 1968. In Aupust 1971, ITs Unit 1 was comverted from an eveporator to cooler for ITS Unit 2. Both units were shut down Jione 1974.

(11) Currently inoparative. The 242-5 Eveporator-Crytallizer was started up Movember 1973, and shut doun Morch 1900, when its processing eempoien wes completed. It is in standby mode with no future aission. This oveporator operates under a veeun, employing eveporative concentration with subecequent crystallization and precipitation of ealt crystals.

(12) Currentiy ineperative. The 242-A Evaporator-Crystallizer was started up March 1977, and shut doun April $19 \% 9$ becase of regulatory issues, and has remined shut down for subsequent uparading. This eveporator cperates uncar a vecum, coploying eveporative concentration with subsequent erystallization and precipitation of ealt eryetate (forming ealtcake).

(13) Currently inoperative. Additionol concentrotion of mastes was completed by using the concentrators at REDOX and I Plent. The REDOX concentrotor wes ueed from July 1967 to June 1972, while the B plent concentrator was used from July 1967 to february 1968.

(14) Waste generated for training and testing purposes prior to Eveporator restart. 
- $H H C-E P-0182-69$

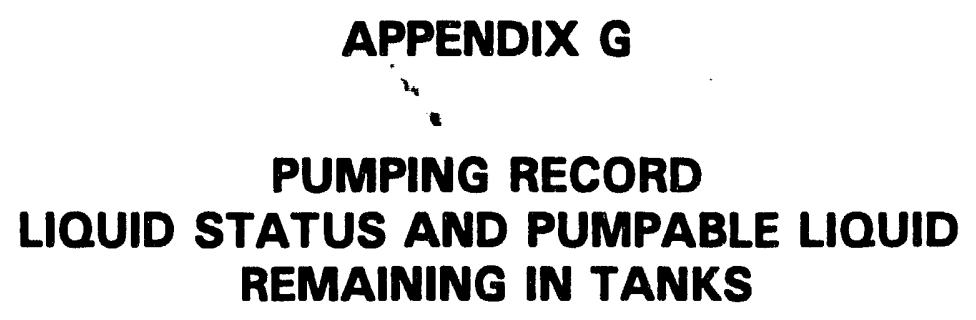

G-1 
WHC-EP-0182-59

This page intentionally left blank. 
TABLE G-1. PUMPING RECORD

(Kgallons)

December 31, 1993

\begin{tabular}{|c|c|c|c|}
\hline $\begin{array}{l}\text { TANK } \\
\text { FARMS }\end{array}$ & $\begin{array}{c}\text { PUMPED } \\
\text { THIS MONTH } \\
\end{array}$ & $\begin{array}{l}\text { PUMPED FY } \\
\text { TODATE } \\
\end{array}$ & $\begin{array}{l}\text { CUMULATIVE } \\
\text { TOTAL PUMPED } \\
1979 \text { TO DATE } \\
\end{array}$ \\
\hline \multicolumn{4}{|l|}{ 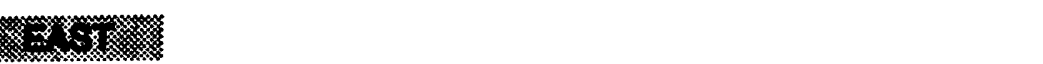 } \\
\hline A & 0.0 & 0.0 & 150.5 \\
\hline AN & N/A & NA & $\mathbf{N A}$ \\
\hline $\mathbf{A P}$ & N/A & NA & NA \\
\hline AW & N/A & NA & NA \\
\hline$A x$ & 0.0 & 0.0 & 13.0 \\
\hline AY & N/A & NA & N/A \\
\hline NZ & NA & NA & NA \\
\hline B & 0.0 & 0.0 & 0.0 \\
\hline$B X$ & 27.7 & 58.7 & 126.5 \\
\hline BY & 0.0 & 0.0 & 1387.4 (a) \\
\hline C & 0.0 & 0.0 & 36.8 (b) \\
\hline 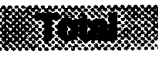 & $x$ & . & S \\
\hline \multicolumn{4}{|l|}{ 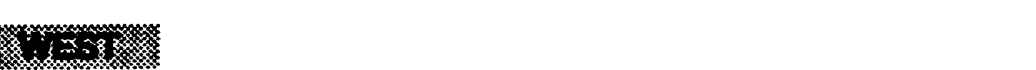 } \\
\hline $\mathbf{S}$ & 0.0 & 0.0 & 791.0 \\
\hline sx & 0.0 & 0.0 & 113.2 \\
\hline SY & N/A & NA & NA \\
\hline $\mathbf{T}$ & 0.0 & 0.0 & 25.3 \\
\hline $\mathbf{T X}$ & 0.0 & 0.0 & 1205.7 \\
\hline$T Y$ & 0.0 & 0.0 & 29.9 \\
\hline $\mathbf{u}$ & 0.0 & 0.0 & 0.0 \\
\hline mon & sonsins & 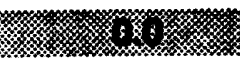 & 装 \\
\hline 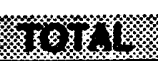 & 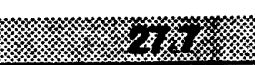 & 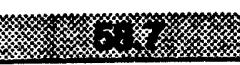 & 3 \\
\hline
\end{tabular}

NA $=$ Not Applicable

(a) The total volume pumped was adjusted by the Single-Shell Tanks Englneer 10 account for the $14 \%$ miscalibration of the constant velocity transmitter and the amount of flush water used. DIL. DLR and PLR volumes were recalculated, based on the obsenved porostity in 102 and 109-BY.

(b) The total volume pumped was recalculated by the Single-Shell Tanks Engineer based on the surface level readings taken after stuutdown of the saltwell pumps on January 28, 1992, in 102-C, 107-C and 110-C, and revised again based on recalculation of flust water additions on January 26, 1993. (The total for the tank farm remains the same, the emount pumped varled among the three tanks). 
TABLE G-2. LIQUID STATUS AND PUMPABLE LIQUID REMAINING IN TANKS

WASTE VOLUMES (Kgallons)

December 31, 1993

\begin{tabular}{|c|c|c|c|c|}
\hline $\begin{array}{l}\text { TANK } \\
\text { FARMS } \\
\end{array}$ & $\begin{array}{l}\text { SUPERNATANT } \\
\text { LIQUID }\end{array}$ & $\begin{array}{l}\text { DRAMABLE } \\
\text { NTERSTITIAL } \\
\text { LQQUID } \\
\end{array}$ & $\begin{array}{c}\text { DRAMMBLE } \\
\text { LQUID } \\
\text { REMAMING } \\
\end{array}$ & $\begin{array}{l}\text { PUMPABLE } \\
\text { LUQUID } \\
\text { REMANING } \\
\end{array}$ \\
\hline \multicolumn{5}{|l|}{ ( } \\
\hline A & 9 & 441 & 450 & 390 \\
\hline AN & 4583 & 37 & 4620 & NA \\
\hline AP & 7269 & 0 & 7269 & N/A \\
\hline AW & 4669 & 159 & 4828 & NA \\
\hline$A X$ & 3 & 370 & 373 & 304 \\
\hline AY & 1720 & 2 & 1722 & N/A \\
\hline $\mathbf{A Z}$ & 1775 & 4 & 1779 & NA \\
\hline B & 15 & 164 & 179 & 80 \\
\hline$B X$ & 30 & 120 & 151 & 85 \\
\hline BY & 0 & 780 & 780 & 598 \\
\hline C & 169 & 143 & 312 & 254 \\
\hline I0.2) & $6 \%$ & 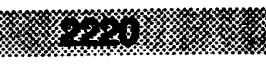 & & (s) \\
\hline \multicolumn{5}{|l|}{ WEST } \\
\hline $\mathbf{S}$ & 58 & 1376 & 1434 & 1201 \\
\hline$s x$ & 63 & 1260 & 1323 & 1178 \\
\hline SY & 845 & 237 & 1082 & NA \\
\hline $\mathbf{T}$ & 45 & 200 & 245 & 178 \\
\hline$T X$ & 5 & 250 & 255 & 0 \\
\hline TY & 3 & 31 & 34 & 0 \\
\hline $\mathbf{U}$ & 168 & 1138 & 1306 & 1104 \\
\hline \% & 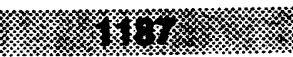 & 紧 & 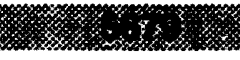 & 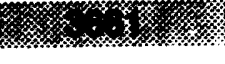 \\
\hline
\end{tabular}

(1) Volume based on 12.5\% (studge waste) and 45\% (saltcake waste) liquid in solid (porosity) value. This is a conservative (high) estimate.

Note: $+1-1 \mathrm{Kgal}$ differences are the result of computer rounding

NA $=$ Not applicable 
WHC-EP-0182-69

\section{APPENDIX H}

\section{CATCH TANKS AND SPECIAL} SURVEILLANCE FACILITIES

H-1 
WHC-EP-0182-69

This page intentionally left blank. 
TABLE H-1. EAST AND WEST AREA CATCH TANKS AND SPECIAL SURVEILLANCE FACILTIES

ACTIVE - still running transfers through the associated diversion boxes or pipeline encasements

December 31, 1993

\begin{tabular}{|c|c|c|c|c|c|}
\hline \multicolumn{6}{|c|}{ Decemioer 31, 1993} \\
\hline FACILTY & LOCATION & PURPOSE (receives waste from:) & $\begin{array}{c}\text { VOLUME } \\
\text { OF } \\
\text { CONTENTS } \\
\text { (Gallons) }\end{array}$ & $\begin{array}{l}\text { MONTOAED } \\
\text { BY }\end{array}$ & REMMAKS \\
\hline
\end{tabular}

EAST AREA

241-A-302-A

241-311-ER

241-152-AX

241-151-AZ

241-154-AZ

244-BX-TKSAP

244-A-TKSIPP

204-AR

417-A

Vent Station Catch Tank

\begin{tabular}{|c|c|}
\hline $\begin{array}{l}\text { A FARY } \\
\text { B PLANT }\end{array}$ & $\begin{array}{l}\text { 151-A DB } \\
\text { 151-ER, 152-EA DB }\end{array}$ \\
\hline AX FARY & 152-AX DB \\
\hline $\begin{array}{l}\text { AZ FAFM } \\
\text { AZ FARM }\end{array}$ & $\begin{array}{l}\text { 152-AZ DA, AZ LOOP SEAL } \\
\text { 102-AZ HTG COL STENM CONDENSATE }\end{array}$ \\
\hline BX COMPLEX & DCFT - RECENES FROM SEVERAL FARMS \\
\hline A COMPLEX & DCFT - RECENES FROM SEVERAL FAFMS \\
\hline $\begin{array}{l}\text { AY FARA } \\
\text { A FARM }\end{array}$ & \\
\hline
\end{tabular}

章

WEST AREA

241-TX-302-C

$241-U-301-8$

241-UX-302-A

241-5-304

244-S-TKSEP

TXFAFM

U FAPM

U PLANT

S FAPM

244-TX-TKRsiap
CROSS Country TPANSFEA Line

154-TX Da

151-U, 152-U, 153-U, 252-U Da

154-UX08

151-S Da

DCFT - RECENES FROM SEVERAL FARMS

DCAT - RECEVES FROM SEVERAL FARIS

Total active facinies 16

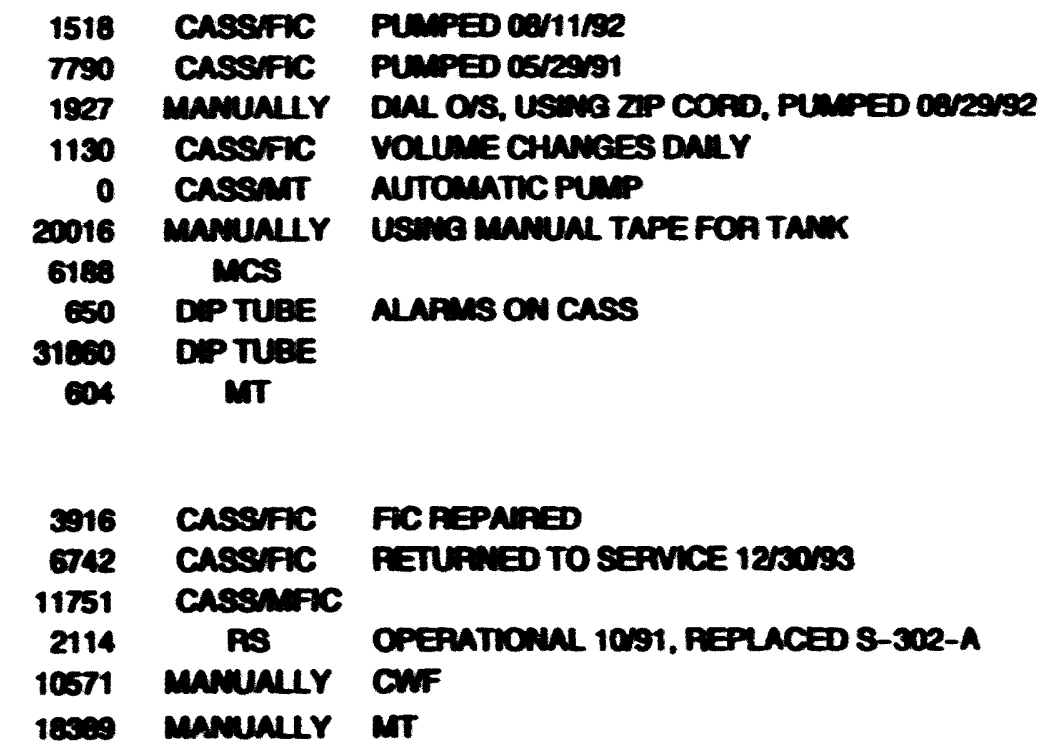

\begin{tabular}{|c|c|}
\hline 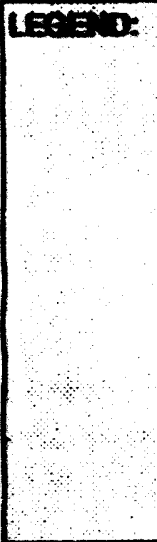 & 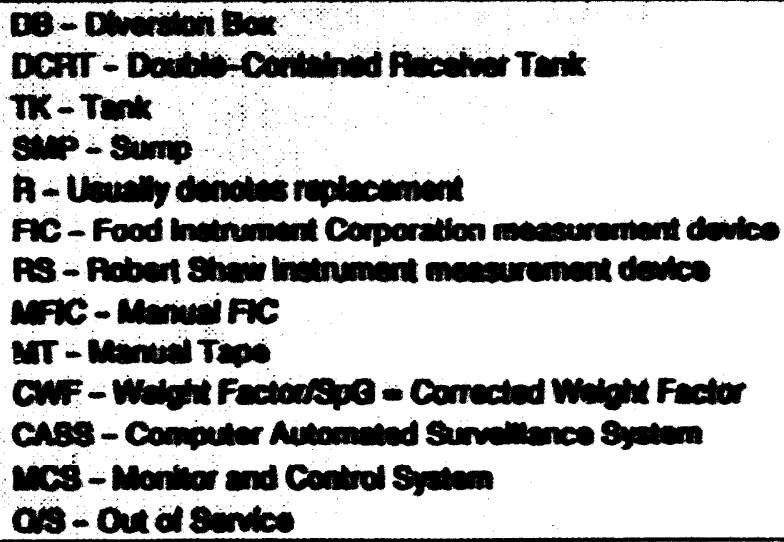 \\
\hline
\end{tabular}


TABLE H-2. EAST AREA CATCH TANKS AND SPECIAL SURVEILLANCE FACILITIES

INACTIVE - no longer receiving waste tranders

December 31, 1983

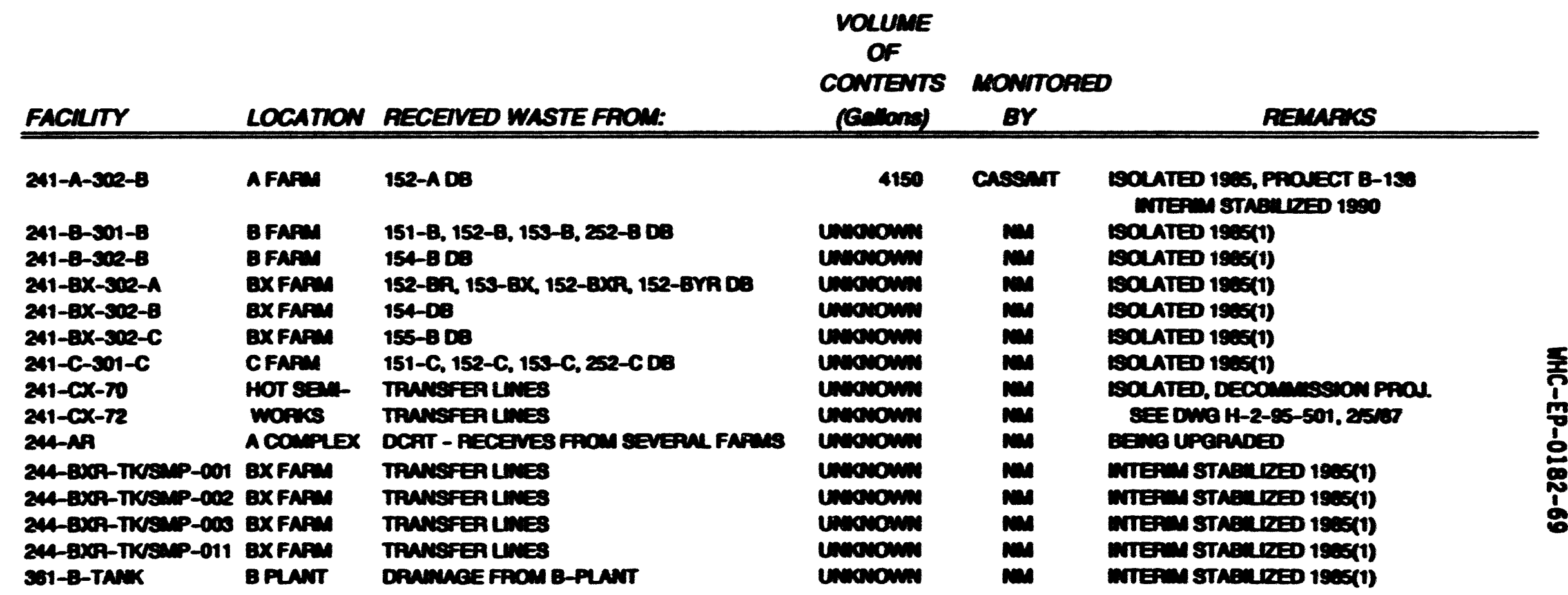

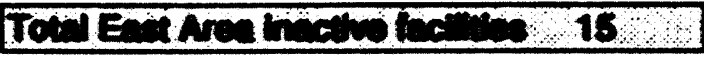

(1) SOURCE: WASTE STOPUGE TANK STATUS \& LEAK DETECTION CPTIEPA document

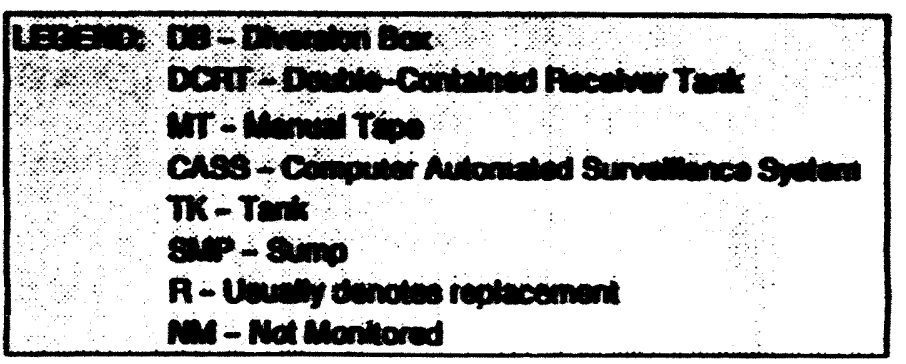


TABLE H-3. WEST AREA CATCH TANKS AND SPECIAL SURVEILANCE FACILITIES

INACTIVE - no longer receiving waste tranders

December 31, 1998

\begin{tabular}{|c|c|c|c|c|c|}
\hline FAcrutr & LOCATION & RECENED WASTE FROM: & $\begin{array}{l}\text { VOLUME } \\
\text { OF } \\
\text { CONTENTS } \\
\text { (Eellone) }\end{array}$ & $B Y$ & REMARIKS \\
\hline $\begin{array}{l}241-8-302 \\
241-8-302-A\end{array}$ & $\begin{array}{l}\text { SFAPAM } \\
\text { SFAFA }\end{array}$ & $\begin{array}{l}240-S-15108 \\
241-8-15108\end{array}$ & 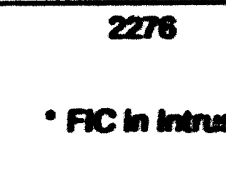 & $\begin{array}{l}\text { Cassiric " } \\
\text { cassiric - } \\
\text { on mods }\end{array}$ & 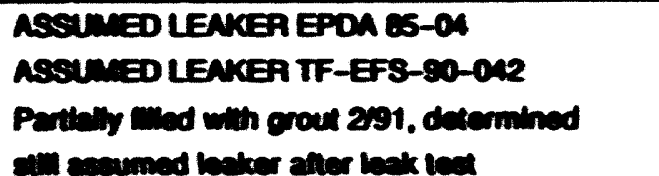 \\
\hline 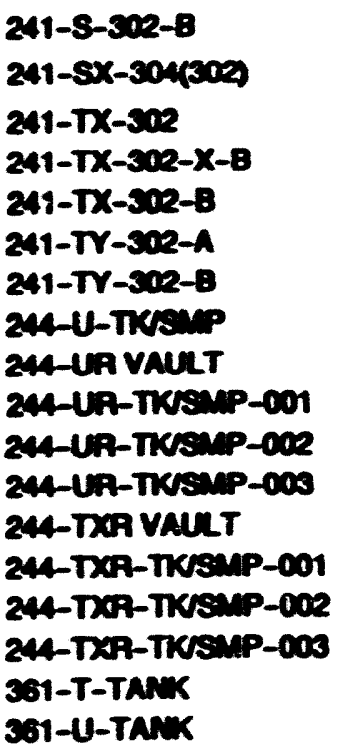 & 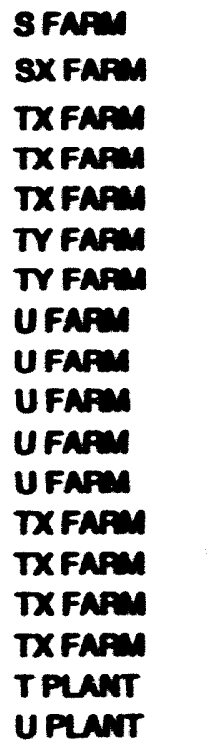 & 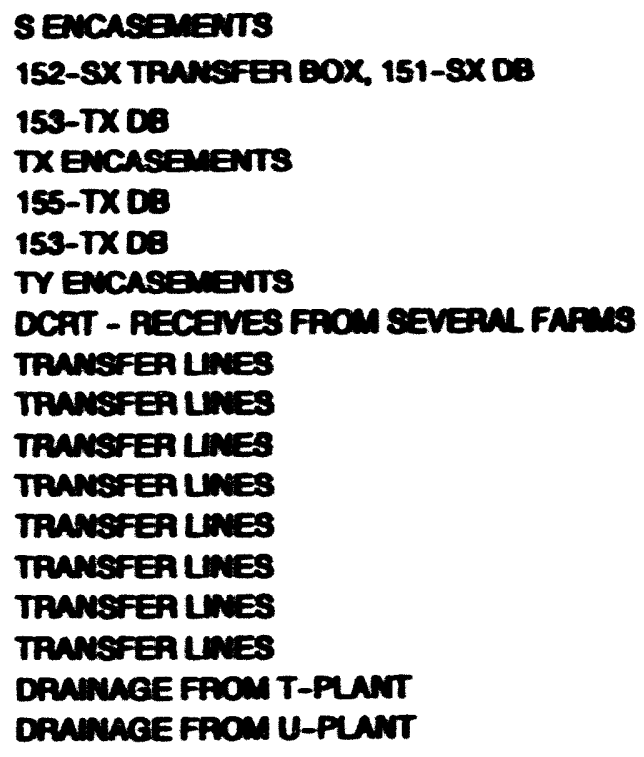 & 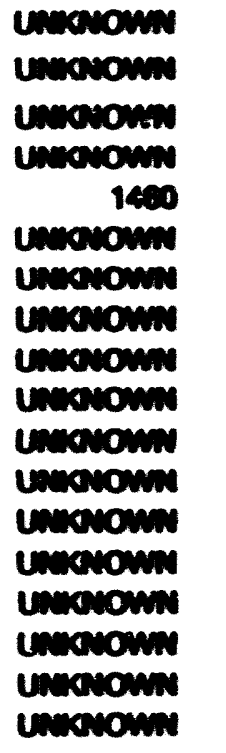 & 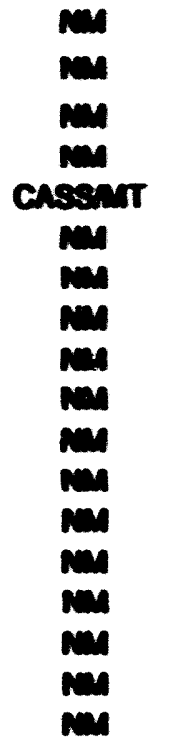 & 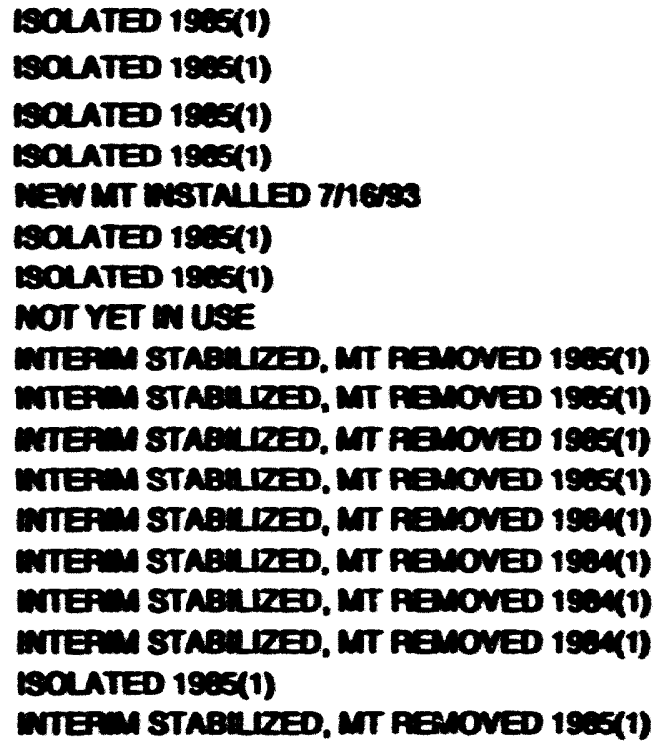 \\
\hline
\end{tabular}

Totd Weat Arou hinction focinion 20

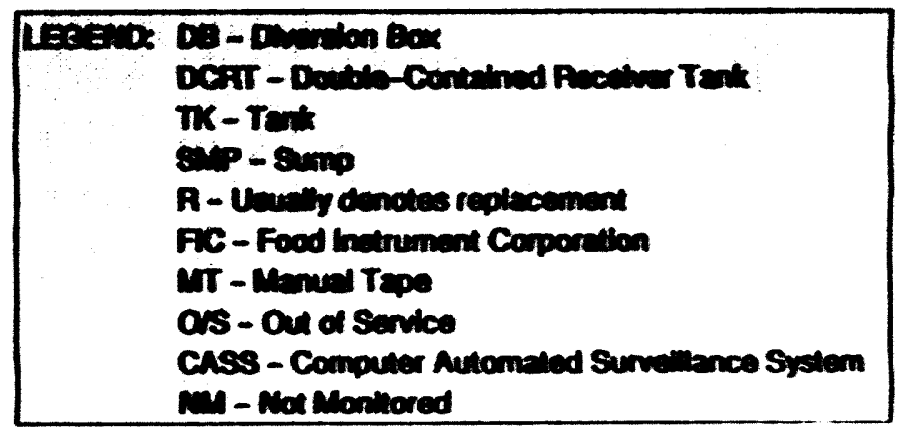


WHC-EP-0182-59

This page intentionally left blank. 
WHC-EP-0182-69

\section{APPENDIX I \\ LEAK VOLUME ESTIMATES}




\section{WHC-EP-0182-E9}

This page intentionally left blank. 
WHC-EP-0182-60

TABLE I-1. SINGLE-SHELL TANK LEAK VOLUME ESTIMATES (4) (Sheot 1 of 6)

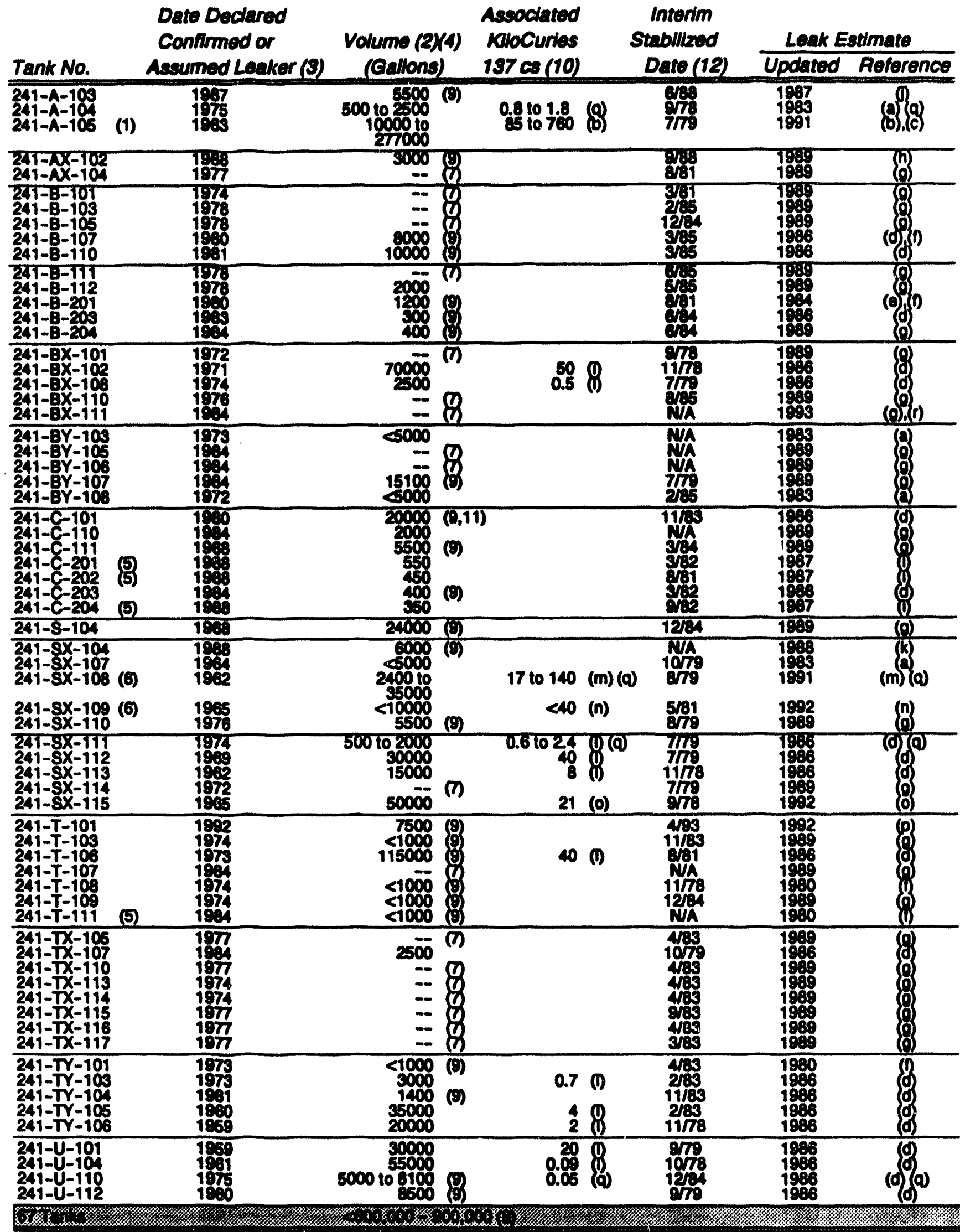

NA = not applicablo (not yet interim atabilized)

FOOTNOTES: SEE NEXT PAGE 
MHC-EP-0182-69

\section{TABLE 1-1. SINGLE-SHELL TANK LEAK VOLUME ESTIMATES (Sheet 2 of 4)}

\section{Footnotes:}

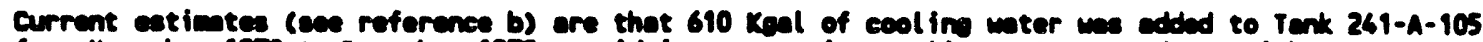

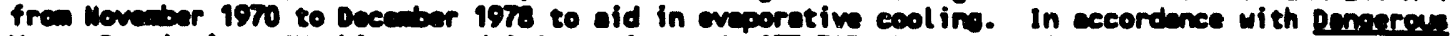

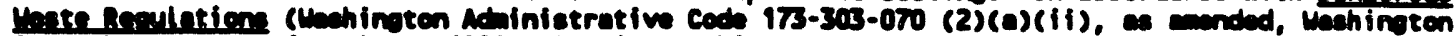

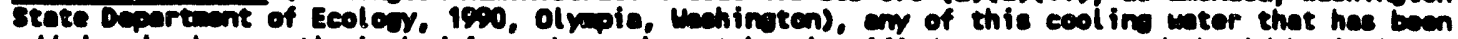

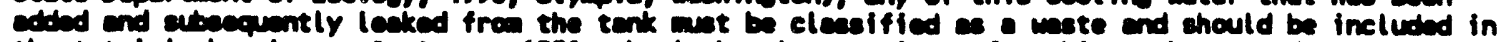

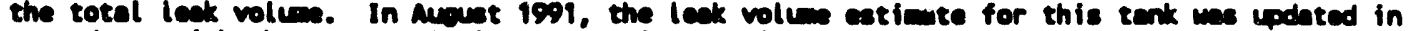
eceordonce with the unc reculations. Previou catientes excluded the cool ino water leaks frem the

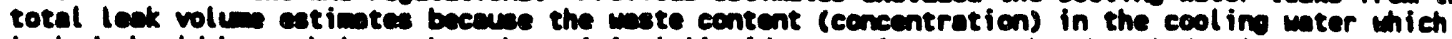
leaked should be wuch leas then the original liquid maste in the tank (the sludes is relotively inaluble). The totol leak volume estimate in this report (10 Keal to $277 \mathrm{Koal}$ ) is besed on the following (ewe References).

1. Deference (b) centains en catime of $5 \mathrm{keal}$ to $15 \mathrm{Keal}$ for the initial leak prior to areust ises.

2. Reference (b) centain en eatiente of $5 \mathrm{keal}$ to $30 \mathrm{Keal}$ for the leak mille the tenk wes being sluleed frem Auruet 1963 to Movmber 1970.

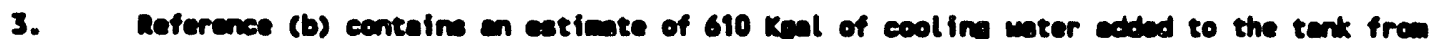

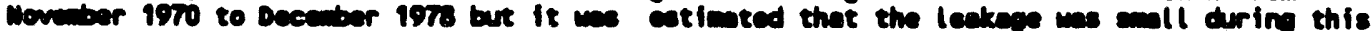
peried. This referenes centaine the stotement seufficient heot wes censeroted in the tenk

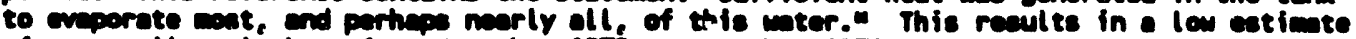

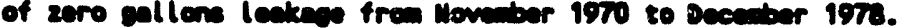

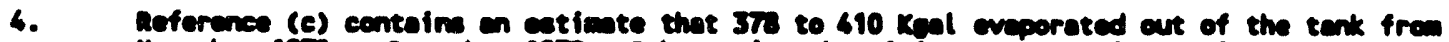

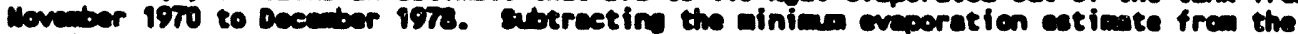

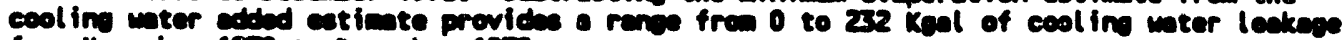
fren Noviber 1970 to becenber 1976.

Leufitionte

Prior to Muset 1968 Aurust $194 \mathrm{a}$ to Movember 1970 Novedber 1970 to Decenber 1976 Totals

$\begin{array}{rr}5,000 & 15,000 \\ 5,000 & 30,000 \\ 10,000 & \frac{2790,000}{277,000}\end{array}$

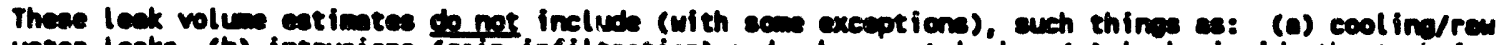
weter leaks, (b) intrusions (roin infiltration) end ubecquent leaks. (c) leaks inside the tank farm but not throwh the tank linor (eurfece leaks, pipoline leaks, leaks ot the joint for the overfleu or fill lines, etc.), and (d) leaks frow eatch tentes, diversion baxes, enceseamente, etc.

In may caces, - leak was supected lone before it un Identified or confirmod. For exemple,

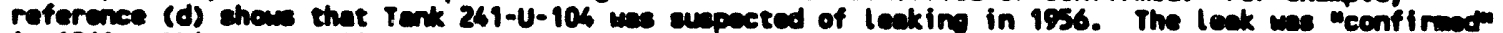

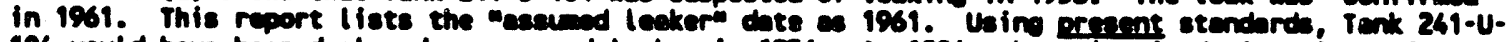

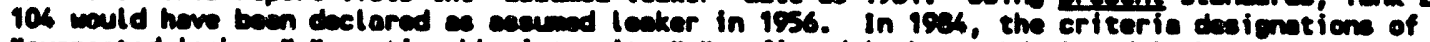

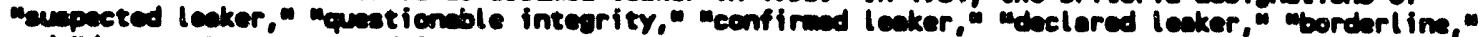

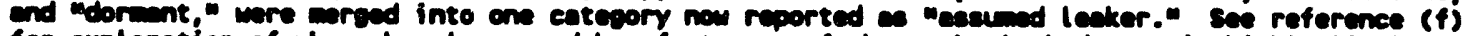
for explanation of men, how lang, and how fast case of the tenks leaked. It is highly likely that there hove been undetected leake frem single-shall tents beceuse of the nature of their design end instrumentation.

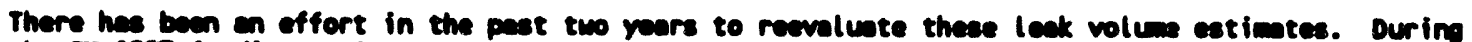
the FY 1993 funding revieus, this revveluntien of leak volumes wes given a priority wich reaulted in this cetivity ne lenear being fundod. The priority vereus fundino will be reevelunted es part of the prior to iv $19 \%$ butert plening.

The leak volum eatinte date for these tanks is bofore the mdeclered leckern date becume the tenk

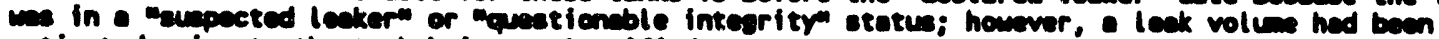
cetibuted prior to the terk being recleasified.

The increasing rediation levels in drymlls and loterals easociated with theas three tenks could be indicative of a centinuing leak of bovement of existing redionucl ides in the coil. There is no conclusive wy to cenfirm theas dbarvetion.

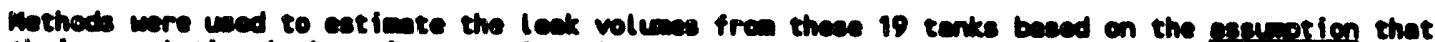

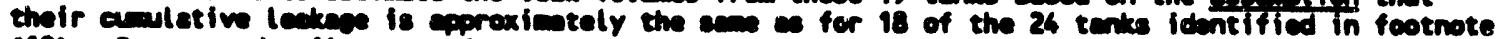

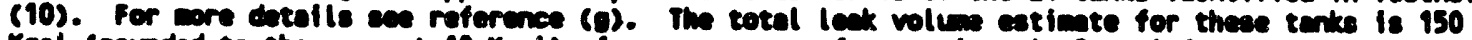

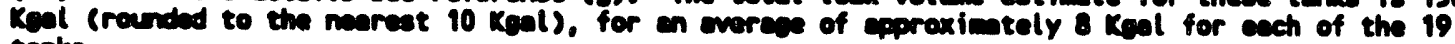
tentes. 


\section{TABLE 1-1. SINGLE-SHELL TANK LEAK VOLUME ESTIMATES \\ (Sheet 3 of 4 )}

(8) The total hes been rounded to the nearest $50 \mathrm{kgol}$. Upporbound volues ware used in many ceace in develeping these cationtes. It is likely thet saim of thees tenks have not ectublly leaked.

(9) Leak volum estivate is beed solely on observed liquid level decreases in these tenks. This is considered to be the wost eccurate method for estivating leak volumes.

(10) The curie content listed is es lleted in the reference docuent and is not docaved to o consistent date; therefore, - caulative total is incppropriate.

(11) Tank 261-C-101 experienced a liquid level decrease in the lote 1960s and was taken out of service

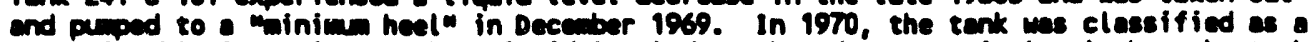
"questionble integrity" tank. Liquid level dota show dacreases in level throwhout the 1970 s end the tenk was ealtwell puped during the 1970s, ending in April 1979. The tenk was reclessified as a "cenfinmed leakere in demury 1900. See reference (q) end (s): refer to reference (s) for informetion on the potential for there to have been leaks frem other C-farm tenks (epecifically, C102, C-103, and (-109).

(12) These dotes indicate when the tenks wre doclared to be interin stabilized. In some cases, the official interin stcbllization docenents were iseund ot - loter dote. Also, in seme ceses, the field work eacociated with interim stebllizotion wa cempleted at en corlier date. 
MHC-EP-0182-69

\section{TABLE 1-1. SINGLE-SHELL TANK LEAK VOLUME ESTIMATES (Sheet 4 of 4 )}

\section{References:}

(a) Murthy, K.S., et al, June 1903, Amesegment of Sinole-shell Tenk Residual Liouid Issues at Hentord site. Mnshington, PULL-6688, Pacific Morthwest Laboratory, Richlend, Washington.

(b) MHC, 19910, Innk 261-A-105 Lenk Agsesfantent, WHC-m-0264, Westinghouse Manford Company, Richland, Washington.

(c) WHC, 19916, Innk 241-A-105 Evnporntion festimte 1970 Throweh 1979, WHC-EP-0410, West inghouse Hanford Compeny, Richlend, Washington.

(d) Smith, D. A.. Jenury 1986, sin-le-shell Ienk Inolntion snfety Analyis Report, SD-Wy-SAR-006, Rev. 1. West inghouse Manford Compeny, Richtand, Washington.

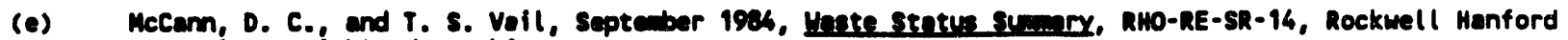
Operations, Richlend, Wechinoton.

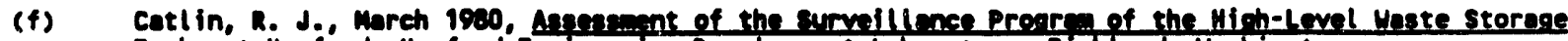

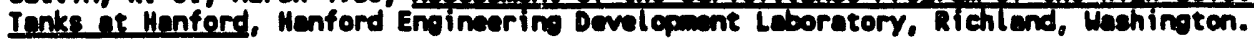

(g) Baunhardt, R. J., May 15, 1909, Letter to R. E. Gerton, U.S. Deppartiment of Energy-Richland Operations office, single-shell Innk Lnk Volvins, Westinchouse Hanford Company, Richland, Wechington.

(h) WuC, 1990a, occurrence Report, surface teml monurement pearnes in single-shnll Ienk 261-Ax-102. MHC-U0-89-023-TF-05, Westinghouse Henford Compeny, Richtend, Washington.

(i) Groth, D. R.. July 1, 1987 , Internal Memoranden to R. J. Eecmardt, Lfouid Level Logege in Tanks 261-c-201, -202 and -20\%, 65950-87-517, Weet inchowe Menford campemy, Richtand, Washington.

(j) Groth, D. R. and 6. C. Oums, May 15, 1987, Internal Meorenden to J. H. Roecker, Ienk 103-A Intescity Evaluation, Westinghowe Menford Cempany, Richlend, Washington.

(k) Capbell, G. D., July 8, 1988, Internal Mesorandem to R. K. Welty, En.inerin Imestiention:

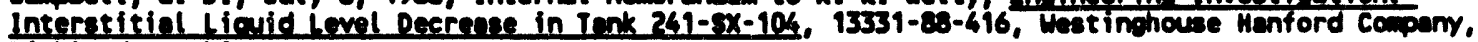
Richlend, Uashington.

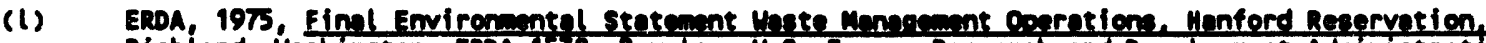
Richlend. Uhahington, ERDA-1538, 2 vols., U.S. Enerey Reseorch and Devolopenent Adtínistrotion, Washington, D.C.

(m) WHC, 1992a, Innk 261-sX-108 Leak Arsengment, MHC-MR-0300, West inghouse Manford Company, Richlend, Washington.

(n) WHC, 1992b, Ienk 261-5x-109 Leak Assengaent, WHC-MR-0301, West inghouse Hanford Company, Richland, Washimiton.

(0) WHC, 1992c, Ienk 241-5X-115 Leak Aseresment, WHC-Ma-0302, West inghouse Henford Company, Richland, Washington.

(p) WHC, 1992d, Occurrence Report, MApporent Decrease in Liquid Level in single shell Underaround Storage Tank 261-T-101, Leak suspected; Imvestigation Cont inving," RL-MHC-TAMKFARH-1992-0073, West inghowe Menford Compary, Richlend, Wachington.

(q)

UMC-1990b, A Mistory of the 200 Aren Tenk Far..., WHC-Mm-0132, Westinghouse Menford Company, Richlend, washinoton.

(r) WHC, 1993, Occurrence Report, single-shell Underoround Mnste storege Tenk 241-BX-111 Surfoce Level pecrense ond Chenoe From steesty state condition, RL-WHC-TAMKFARM-1993-0035, Westinghouse Hanford Compeny, Richtend, Washington.

(s) WHC, 19930, Assensant of Unasurnted zom Redionualide Contemintion Around single-shell ienks 249 -

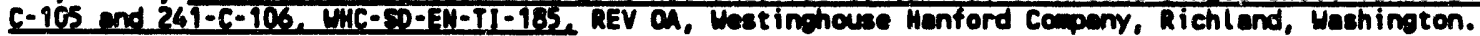


WHC-EP-0182-69

\section{APPENDIX J}

INTERIM STABILIZATION STATUS 


\section{WHC-EP-0182-69}

This page intentionally left blank. 
WiC-EP-0182-60

TABLE J-1. SINGLE-SHELL TANKS INTERIM STABILIZATION STATUS (Sheet 1 of 2) December 31, 1993

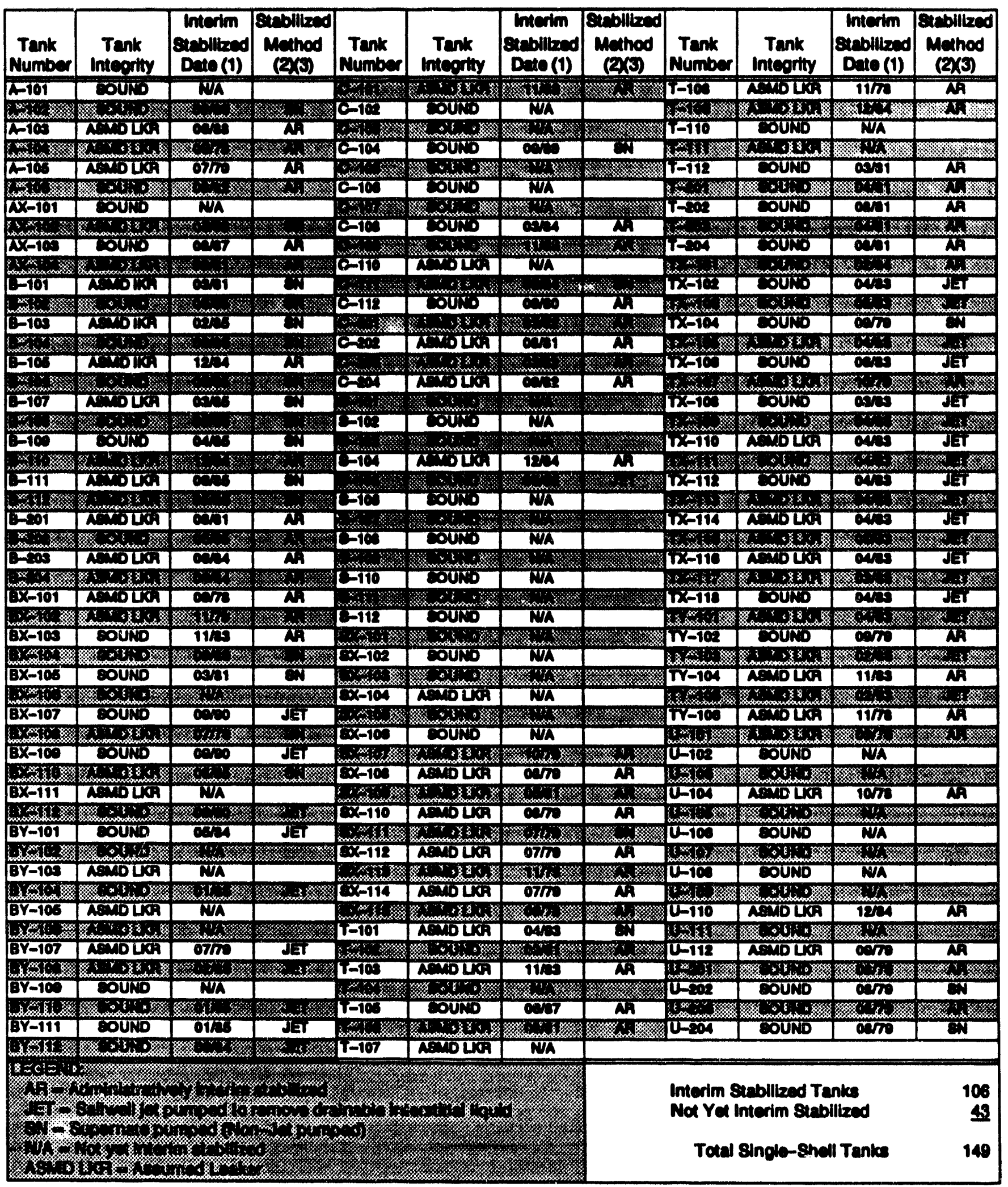

Footnotes: See next page 
TABLE J-1. SINGLE-SHELL. TANKS INTERIM STABILIZATION STATUS

(sheet 2 of 2)

\section{Footnotes:}

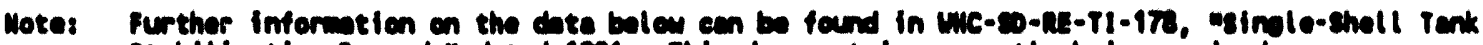
stebillizetion Record," dated 1991. This decenent is eurrently beirs revieed.

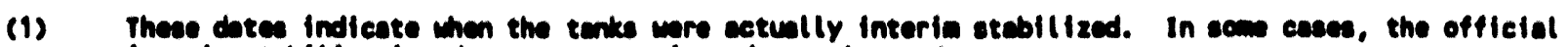
interis stcbllization documente wre lseund ot a later dote.

(2) The following six tente do not wot current cescbliched cupornotent and interetitiol liquid interin

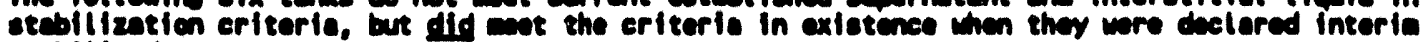
otebllized:

$$
\begin{aligned}
& \text { อ-10., 110. } 111 \\
& \text { T-102, } 112 \\
& \text { U-110 }
\end{aligned}
$$

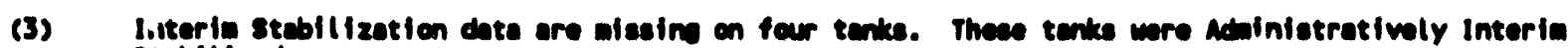
stebllized.

$$
\text { 1-201, T-102, 112, } 201
$$




\section{DISTRIBUTION}

Lumber of cootes

OEFSITE - USA
Conorens of the Untied stathe

House of Representatives

1111 Longworth Butlding

Washington, DC 20515-3703

Ron Wyden, Member of Congress, 3rd District Josh Kardon, Legislative Director

House of Representatives

1431 Longworth House Office Bullding

Washington D. C. 20515

Jay Inslee, Member of Congress, 4th District Atten: Louis J. Alex, Legislative Assistant

19

ע. S. Papertment of Fneroy-findeunetere 1000 Independence Avenue, SW Washington, D. C. 20585

Jim Antizzo

Emile Bernard

EM-36

EM-50

EM-36

H. Calley

H. Eckert

Teresa Fryberger

Sherry Gibson

EM-37

EM-542

EM-55

A. Griffith

EM-36

EM-36

EM-36

D. Gupta

Kenneth Lang

J. C. Lehr

G. Mellinger

C. O'Dell

D. Pepson

J. C. Tseng

S. Woodbury

EM-36

EM-442

EM-36

EM-36

EM-36

EM-36

EH-222

TREV II/364

TREV II

TREY II

TREV II

TREV II

TREV II

TREV II/34I

TREV II

TREV II

TREV II

TREV II/160

TREV II

TREV II

TREV II

TREV II/364

FORS/3G-092

19901 Germantown Rd, Germantown, MD 20585
R. Lasky
J. Psaras
EH-32.1
NS-20 GTN
GTN/G-115
P. Worthington
NS-20 GTN

U. S. Department of Eneroy - Oak Ridge Operations Office P. O. Box 2001

Oak Ridge, TN 37831

W. D. Adams EW-40 


\section{MNC-EP-0182- 69 \\ Distribution - continued}

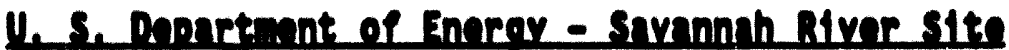

P. O. Box A

Atken, SC 29808

$\begin{array}{ll}\text { C. Anderson } & 707-H \\ \text { Michael Chandler } & 703-H \\ \text { Mazen Shurrab } & 704-H\end{array}$

T. C. Temple

L. SJostrom

W. R. Mest

V. Wheeler

704-8H

704-5

11. S. Environ.ment l Protection Amaray Regton 10

712 Swift Boulevard, Sulte 5

Rtchland, WA 99352

D. R. Sherwood

7

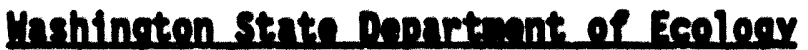
Nuclear \& Mixed Waste Manageanent Program P.0. Box 47600

01 ymp Ia, WA 98504-7600

M. T. Gordon

M. Lerchan

Scott Mckinney

R. Stanley

Library

7601 H. Clearwater 102

Kennewick, WA 99336

S. v. Moore

Office Library

1

Mashington Strte Departant of Henlth

Radiation Protection Section

Industrial Park Building 5, LE-13

01 ympla, WA 98504

\section{A. Conklin}

1

Genernl Accounting office

P. 0. Box 321

Richland, WA 99352

C. R. Abraham

Oreagn State Departinnt of Ennray.

625 Marion St. N.E.

Salem, OR 97310

Janet Franco 


\section{A. . INC-EP-0182-69 \\ Distribution - continued}

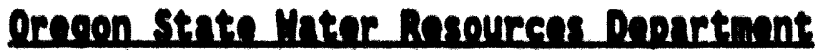
Ground Water Hanford Studtes 3850 Portl and Road Salem, OR 97310

R. O. Patt

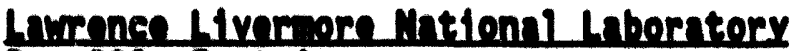
Box 808, East Avenue

Livermore, CA 94550

B. C. Hudson $\lfloor-22\rfloor$

5

onk Riden Mintional L borntory

P. 0. Box 2009

Oak Ridge, TN 37831-6385

C. Forsberg

MS-6495

T. S. Kress

Bldg 9108

MS-8088

Chemical Technology Division

P. 0. Box 2008

Emory D. Collins

C. Pht1 McGinnts

Dr. Jack Watson

Los Almos Mntionnl Laborntory

P. 0. Box 1663

Los Alamos, NM 87545

\section{Stephen Agnew}

Group INC-14

Phyllis Baca

T. Larson

Sylvia Lee

J-514

A. Nuels

C-915

H. Sullivan

K-557

Dr. Reed Jensen

$N-6$

$N-6$

$\mathrm{J}-565$

5

Brookhnyen Mationnl Laboratory Upton, NY 11973

K. K. Bandyopadhyay, Bldg 475-C

M. K. Kalsser, Bidg 475-C

P. D. Kalb, Bidg. 703

M. Reich, Bidg 475-C

J. R. Weeks, B1dg 197-C

1 Brookhnyen Mntionsl Lboratory

1409 Jan Drive

Wilmington, DE 19803

Michael Streicher 
MHC-EP-0182-69

Distribution - continued

2

Aroonne Mntionnl Leborntopy

9700 South Cass Avenue

Argonne, IL 60439

Dr. Martin Steindler

Dr. George Vandergrift

3

Sindin Mntional Leboritortes

1515 Eubank, NE

P. O. Box 5800

Aibuquerque, N 87185

Scott Slezak, Division 6471

Leon D. Chapman, Progran Manager

Industrial Waste Reduction Progran

Or. Margaret Chu, Organ 16622

1

Mnsmahurntts Institute of Inchnoloay

77 Massachusetts Avenue

Cambridge, MA 02139

Mujid S. Kazimi

Professor and Head

Department of Nuclear Engineering

2

mon Internntionnl. Ine.

20030 Century Blvd, Suite 101

Germantown, No 20874

P. Kiang

K. J. Mahoney

3

SAIC

Trevion 1, Suite 300

20300 Century Bivd.

Germantown, MD 20874

J. Bunting

J. R. Pearring

R. A. Wullaert

1

102 Windhan Road

Oak Ridge, TN 37830

D. 0. Compbell

1

1845 Terminal Drive, Suite 130

Richland, WA 99352

J. Mishima 


$$
\begin{gathered}
\text { MAC-EP-0182-69 } \\
\text { Distribution - continuad }
\end{gathered}
$$

555 Quince Orchard Road

Gaithersburg, MD 20878

\section{Paul Szerszen}

$1 \quad$ Harvard Universtty

295 Upland Avenue

Newton Highlands, MA 02161

Melvin First

1

Syreause University

334 Hinds Hall

Chemical Engineering \& Materials Science Department Syracuse, NY 13244

Dr. Larry Tavlarides

1 Inivarsity of Mashinaton Chemical Engineering Department

Benson Hall, BF-10

Seattle, WA 98195

Professor Gene Noodruff

Confederntad Tribes. Unatille Indion Reservation

P. 0. Box 638

Pendieton, OR 97801

Rick George

5

Mest Yelley Muclear Services co.

P. O. Box 191

West Valley, NY 14171

K. K. Gupta

S. Ketola

MS-49

D. K. Ploetz

MS-191

Ram Shukla

MS-305

Don Stroud

K \& Engineering \& Consulting Corporation

Suite 500

2001 L St. NW

Washington, D. C. 20036

Ryan Gill 
WHC-EP-0182-69

Distribution - continued

Dafense Mucienr Facilities snfoty Boned 625 Indiana Ave, N. W., Suite 700

Washington, D. C. 20004

Lester Clemons

Suite 700

Timothy Dwyer

Steven Stokes

Richard Tontodonato

Mest inghouse Idahe Nuclear Corporation

P. 0. Box 4000

Idaho Falls, ID 83404-4000

B. Griebenow

A. P. Hoskins

MS-5104

Dr. Terry Todd

MS-5217

1

C. Abrams

1987 Virginia Drive

Idaho Falls, ID 83404

1

Fred N. Carlson

6965 North 5th West

Idaho Falls, ID 83401

1

Donald T. Oakley

555 Quince Orchard Road

Gaithersburg, MD 20878

1

Dr. A. Veletsos

Department of Civil Engineering

Rice University

P. O. Box 1892

Houston, TX 77252

2

Mestinghouse Flectric Corporation

$1801 \mathrm{~K}$ Street $N W, 8$ th floor

Washington DC, 20006

Kevin Billings

6 Gateway Center

Pittsburgh, PA 15222

Steve Green, Manager

Westinghouse Environmental Affairs

Sestinohouse Materials Company of Ohio

P. O. Box 398704

Cincinnati OH 45239-8704

David L. Jacoboski

Senior Engineer, Technology Demonstration 
WHC-EP-0182-69

\section{Distribution - continuod}

Unst inghouse Sayannah River Company

P. 0. Box 616

Aiken, SC 29802

D. M. Barnes, 773-41A

J. R. Chandler, 703-H

P. d'Entremont

F. G. McNatt, 704-8A

Dr. Major Thompson

1991 S. Centennial Ave.

Aiken, SC 29803

L. A. Wooten

Institute for Fneroy and Environmental Reseach

6935 Laurel Avenue

Takoma Park, MD 20912

Dr. Arjun Makhijani, President

1

SPAR Aerospras Lted.

20 Avon Meadow Lane, Suite 220

Avon, CT 06001

Peter W. Kruse

Advanced Technology Systems Division

1

Redzone Robottcs. Ince

2425 Liberty Ave

Pittsburgh, PA 15222-4639

David W. White

6

Mational Research Counctl. National Academy of Sciences

2101 Constitution Ave., N. W.

Washington D. C. 20418

Robert S. Andrews, Senior Staff Officer

Board on Radioactive Waste Management

1

Converse Consultants

18 W. Mercer Street, Suite 300

Seattle, WA 98119

David Stanley

1

Brown calduell

100 W. Harrison

Seattle, WA 98119

Hal Cooper 
HHC-EP-0182-69

- Distribution - continued

Benton County Department of Emergency Management

P.0. Box 6144

Kennewick, WA 99336

Gary Pira

1

Boyay Northwest Inc.

660 Swift, Suite D

Richland, WA 99352

T. J. McLaughl in

Oean Environmental Technology

655 Montgomery Street, Suite 1000

San Francisco, CA 94111

Mike Bailey

1

GEC ALSTHo." Engineering Systems

P. 0. Box 1274

Richland, WA 99352

J. W. Riddington,

Vice President, Nuclear Marketing

1

T. S. Elleman

North Carolina State University

Department of Nuclear Energy

P. O. Box 7909

Raleigh, NC 27606

1

Mike Lingle

Stone \& Webster

7677 E. Berry Ave

Englewood, CA 80111

1

Bryant Mather

Corps of Engineers

HESSV-Z

3909 Halls Ferry Rd

Vicksburg, MS 39180-6199

1

Paul Shewmon

Prof. Metallurgical Engineer

Ohio State University

2477 Lytham Road

Columbus, $\mathrm{OH} 43220$ 
WHC-EP-0182-69

$$
\text { Distribution - continued }
$$

Maste Management External Advisory Committee Members

Dr. Frank L. Parker

Professor of Environmental and Water Resources Engineering

Vanderbilt University

P. O. Box 1596, Station B

Nashville, TN 37235

Dr. Bruce R. Kowalski

Professor of Chemistry, Co-director of Center for Process

Analytical Chemistry

University of Washington

Chemistry Department, Bidg 10

Seattle, WA 98195

Dr. Greg R. Choppin

Professor of Chemistry

Florida State Uriversity

Department of Chemistry, B-164

Tallahassee, FL 32306

Dr. Chester Grelecki

President, Chief Scientist

Hazards Research Corporation

200 Valley Road, Suite 301

Mt. Arlington, NJ 07856

Dr. Alfred Schneider

Professor Meritus of Nuclear Engineering

Georgia Institute of Technology

5005 Hidden Branches Dr.

Dunwoody, GA 30338

Dr. Gary Powers

President

Design Science, Inc.

163 Witherow Road

Sewickley, PA 15143

Joseph S. Byrd

University of South Carolina

Department of Electrical and Computer Engineering

Swearingen Engineering Center

Columbia, SC 29208

William R. Prindle

1556 Crestline Drive

Santa Barbara, CA 93105 
MHC-EP-0182 69

Distribution - continued

3

Ames Laboratory

7 Spedding Hall

Iowa State University

Ames, IA 50011

Bill Haas

R. B. Thompson

Dr. Paut Wang

1

RKK Lted.

16404 Smokey Pt. Blvd, Suite 303

Arlington, WA 98223

Chris Reno

1

MACTEC

8320 Centerbrook Place

Alexandria, VA 22308

Stan Blacker

1

Engineering-Sclence. Inc.

1955 Jadwin Ave, Suite 470

Richland, WA 99352

Matt Sakach

1

EBASCO Services. Inc.

1201 Jadwin Avenue, Suite 202

Richland, WA 99352-3429

F. J. Young

1

VIIL Systems. Inc.

3481 Dayton-Xenia Road

Dayton, OH 45431-0299

E. McDaniel

1

Nuclear Consulting Services. Inc.

7000 Huntley Road

P. O. Box 29151

Columbus, OH 43229

Dr. J. Louis Kovach

Battelle Laboratories

505 King Avenue

Columbus, OH 43201-2693

Rob Taylor Jr., P.E.

Rm 13-6-016 


$$
\text { Distribution - continued }
$$

Portland General Electric Co.

121 S. W. Salmon St.

Portland, OR 97204-2991

Wayne Lei, 3WTCBRO5

3

RUST Geotech Inc.

P. 0. Box 14000

Grand Junction, CO 81502-5504

Brian Mathis

Michael C. Butherus

John R. Duray

1

ICF Kaiser Engineers

1800 Harrison St.

Oakl and, CA 94612-3430

Chris G. Whipple, Ph.D

1

Meptune \& Company

1505 15th St., Suite B

Los Al amos, NM 87544

Randy Ryti

1

John Hopkins University

1714 Eutaw Place

Baltimore, MD 21217

Morton Corn

1

Roberts Engineering Services. INC. 1980 East Ocean Boulevard

Stuart, FL 34916

Dr. Paul Roberts

1 Columbia River United Technical Consultant P. 0. Box 912

Bingen, WA 98605

Gregory de Bruler

1

Government Accountability Project

West Coast Office

1402 Third Avenue, Suite 1215

Seattle, WA 98101

Thomas E. Carpenter, Director 
MHC-EP-0182- 69

\section{Distribution - continued}

Heart of America Northwest

1305 Fourth Avenue

Cobb Building Suite 208

Seattle, WA 98101

Gerald M. Pollet, Executive Director

1

Dr. Viorica Lopez-Avila

Midwest Research Institute

625-B Clyde Ave

Mountain View, CA 94043

1

Los Alamos Technical Associates

8633 W. Gage Bivd.

Kennewick, WA 99336

D. S. Delorenzo

1 Yakima Indian Nation

Environmental Waste Program

1933 Jadwin Ave, Suite 110

Richland, WA 99352

John Brodeur

\section{OFFSITE - FOREIEN}

1

British Nuclear Fuels Ltd

Risiey Warrington

Cheshire WA3 6AS

United Kingdom

Howard A. Edwards

1

Ricardo Hitec Ltd

Club street Works, Bamber Bridge

Preston, PR5 6FN

United Kingdom

P. K. J. Smith

1 Telerobot
Consorzio Telerobot
Via Hermada 6
16154 Genova, Italy
Bruno Sessarego 


\section{MHC-EP-0182-69 \\ Distribution - continued}

4

SEN

1, rue des Herons, Montigny-le-Bretonneux

78182 Saint-Quentin-en-Yvel ines Cedex,

France

Serge Merlin

Gilles Clement

Bernard Tousant

Eric Tchemitcheff

2

CEA - Saclay

DCC/DIR

$B a ' t 121$

91190 GIF/Yvette Cedex

France

G. Baudin

R. Atabek

1

SDinS-Chaudronnerie Blanche

B.P. 4-F-38160 Saint-Romans

France

Phillipe Monneau

1

GEA-Cadarache

SGDC/STDE

Boite Pastale 313

13115 Saint Paul Les Durance

France

Magali Ranchoux

1

Hans Mal ischmiller GmbH

D-7778 Markdorf/Bodensee

Germany

Wolfgang Walischmiller

ONSITE
J. J. Anttonen
G. E. Bishop
R3-73
K. W. Bracken
R3-72
S. T. Burnum
R3-73
R. C. Cullison
R3-74
J. J. Davis
A5-55
L. Erickson
R3-74
R3-74
R. E. Gerton
R3-72
M. Glasper
R3-72
W. F. Hendrickson
R3-72 


\section{MHC-EP-0182-69 \\ Distribution - continued}

R. L. Higgins

R. D. Hildebrand

A. Hon

P. E. LaMont

T. Noble

L. E. Petersen

G. W. Rosenwald

G. H. Sanders

E. J. Senat

A. B. Sidpara

A. D. Toth

D. L. Vieth

D. J. Williams

J. K. Yerxa

Reading Room
R3-72

A5 -55

R3-72

R3-74

R3-72

R3-72

R3-72

R3-74

R3-72

R3-73

R3-72

R3-73

R3-72

A5-15

A1-65

BNFL INC.

Dr. Mike Mckeon

G3-02

Stone \& Hebster Enginearing Co.

E. L. Richards

R2-83

2

MACTEC

\section{J. Janus}

G6-18

L. Soler

A4-35

1

EBASCO

C. T. Anderson

G3-02

\section{Pacific Northwest Laboratories}

D. N. Anderson

K7-34

W. J. Apley

D. B. Baird

$\mathrm{K} 1-73$

R. M. Bean

D. W. Bennett

P. R. Bredt

J. C. Brown

S. A. Bryan

L. L. Burger

J. B. Colson

J. M. Creer

P. G. Eller

J. F. Fletcher

K7-34

P8-08

K5-17

P7-25

K7-22

P7-25

P7-25

K5-10

G6-01

B1-40

E. A. Flores

L. K. Holton Jr.

K7-97

K3-08

P7-43

V. L. Hunter

K7-94

P. R. Hrma

P8-37

J. Janata

K2-12 
MHC-EP-0182-69

Distribution - continued

B. M. Johnson

KI-78

E. O. Jones

P8-38

D. E. Larson

G3-02

S. G. McKinley

P7-22

B. D. McVeety

K6-63

I. C. McVeety

K7-22

P. J. Mellinger

P7-22

B. E. Opitz

K6-79

M. D. Patridge

K7-94

M. S. Peffers

R. K. Quinn

K7-94

H. G. Richmond

K1-73

P7-41

J. M. Robbins

P7-22

R. D. Scheele

P7-25

E. A. Schmieman

B1-40

R. C. Schrotke

P7-18

P. A. Scott

P7-19

D. A. Seaver

$\mathrm{KI}-73$

J. C. Spanner

K2-05

J. L. Straalsund

$\mathrm{K} 1-79$

K. L. Steinmaus

K6-84

J. L. Swanson

P7-25

K. M. Tominey

KT-97

R. S. Hegeng

K7-97

P. D. Whitney

K7-34

T. W. Wood

K6-47 Sciences Richland/ICF Kaiser Hanford Company
A. T. Alstad
$R 1-49$
W. T. Alumkal
R2-52
R. P. Anantatmula
R2-11
J. D. Anderson
J. N. Appel
N3-11
I. J. Aust in
S4-58
H. Babad
T4-01
R2-78
J. K. Bajwa
H4-63
R. A. Ballou
54-58
A. D. Bates
T. J. Bander
L. L. Barry
T3-01
HO-33
R1-67
G. D. Bazinet
L4-71
L. Bedford
55-07
D. L. Becker
H5-57
D. B. Bechtold
T6-09
R. V. Berg
M. V. Berriochoa
R4-02
P. K. Bhatia
B. D. Biddle
K. R. Birney
D. L. Bjorklund
B3-30
S4-58
(6)
55-14
HO- 40
S6-01
R1-62
J. E. Bjorklund
R1-17 


\section{Distribution - continued}

D. C. Board

K. D. Boomer

G. L. Borshetm

V. C. Boyles

D. A. Bragg

D. R. Bratzel

W. R. Brooksher

T. M. Brown

R. G. Brown

J. H. Bussell

J. A. Caggiano Jr

J. W. Carey

R. J. Cash

T. Chiao

G. Christensen

K. L. Chubb

G. J. Coleman

J. C. Conner

R. B. Conrad

F. M. Coony

W. L. Cowley

C. Crawford

N. R. Croskrey

G. M. Crummel

J. M. Cruse

D. S. Cunningham

S. A. Davidson

J. S. Davis

S. J. Dechter

C. Defigh-Price

T. A. Demitruk

C. J. Denson

D. R. Dickinson

S. E. Dieterle

L. F. Dougherty

J. G. Douglas

G. L. Dunford

R. F. Eggers

D. R. Elitingson

F. W. Ellis

D. B. Engeiman

R. W. Ewert

S. D. Estey

W. G. Farley

J. E. Fasso Jr.

K. O. Fein

L. A. Fort

K. D. Fowler

S. B. Fowler

C. R. Fox

G. L. Fox, Jr.

H. P. Fox

G. T. Frater

E. A. Fredenburg
S1-57

H5-49

R2-11

$R 1-49$

$R 1-49$

L5-31

(4-0)

R2-12

R2-14

L7-06

H6-06

S5-07

R2-78

LO-06

H4-2I

L6-86

T4-07

H4-61

H5-09

H6-07

H4-6I

R3-01

R1-30

R1-51

L5-63

SO-02

K6-26

H4-64

R2-54

R2-31

H5-36

L4-95

L5-31

S2-20

H4-63

L5-55

RI-51

S4-58

H5-37

B4-52

R2-18

RI-19

R2-11

H4-62

S3-10

H4-63

S4-57

R2-11

LO-24

S4-60

L5-01

R2-88

RI-51

S4-55 
J. R. Freeman-Pollard

R. T. Franch

J. C. Fulton

K. A. Gasper

G. J. Gauck

C. J. Geier

P. W. Gibbons

R. L. Gllchrist

D. A. Gilles

S. D. Godfrey

D. E. Good

D. J. Green

A. Greenberg

P. R. Golberg

P. Greenbaum

J. M. Grigsby

R. D. Gustavson

K. D. Haggerty

L. E. Haif

v. W. Hall

C. S. Haller

D. W. Hamiliton

K. L. Hampsten

B. M. Hanion (30)

J. M. Hanson

H. D. Harmon

M. S. Harrington

J. P. Harris III

G. A. Harvey

F. J. Heard

D. A. Healey

J. M. Henderson

D. W. Hendrickson

E. G. Hess

M. C. Higginson

B. M. Hisaw

M. J. Holm

J. D. Hopkins

B. K. Horsager

J. H. Huber

J. L. Huckaby

A. L. Huegel

J. V. Hurley

J. J. Huston

J. E. Irvin

M. N. IsI am

M. T. Jansky

G. D. Johnson

J. L. Juette

R. A. Karnesky

D. L. Kelly

R. A. Kirkbride

P. F. Kison

C. A. Kuhiman
H6-03

E6-61

R2-31

ᄂ6-29

R]-51

R2-50

$54-58$

L5-63

S2-14

RI-51

S3-97

H5-53

S2-66

B4-08

55-20

H4-62

R1-51

T4-09

E6-25

R2-85

66-04

T6-02

(4-7)

RI-80

R2-85

R2-52

B4-53

S4-55

B4-52

HO-34

T4-07

S4-55

L5-55

R3-09

A4-25

RI-62

RI-80

R2-11

B5-24

RI-49

R2-78

E6-31

R4-02

S1-54

R2-85

R3-08

H6-26

R2-78

G6-56

HO-39

S5-20

S4-58

S2-45

B3-30 


\section{Distribution - continued}

N. W. Kirch

D. B. Klos

A. G. Krasopoulos

M. Kunmerer

M. J. Kupfer

E. C. Ladd

D. R. Lance

J. L. Lee

J. M. Light

D. C. Lint

B. H. Lueck Jr.

P. J. Mackey

G. T. MacLean

M. K. Mahaffey

R. M. Marusich

V. D. Maupin

T. B. MeCall

J. D. MeCormack

K. S. MeCullough

M. H. McGrath

M. A. Mclaught in

J. P. Menard

W. C. Miller

N. J. Milliken

W. J. Millsap

G. J. Miskho

J. R. Mobley

T. Moleff

S. R. Morgan

K. L. Morris

J. P. Mullally

L. D. Muhlestein

R. L. Nelson (4)

R. L. Newell

D. J. Newl and

R. Ni

T. W. Oden

P. C. Ohl

P. A. Olsen

D. L. Osowski

D. B. Pabst

A. L. Pajunen

R. B. Pan

I. G. Papp

L. D. Parchen

G. L. Parsons

M. A. Payne

L. T. Pedersen

L. D. Pennington

S. R. Pierce

R. S. Poptelarczyk

E. A. Porter

T. B. Powers

R. K. P'Pool
R2-11

So-14

A5-55

H4-62

H5- 49

RI-19

SO-09

R2-36

B4-08

H3-56

R3-12

B3-15

S4-58

L4-73

H4-60

$\mathrm{NI}-73$

HO-33

[5-31

H4-70

S4-58

H5-09

R2-40

S4-55

H4-62

H5-68

R2-50

H5- 49

RI-30

R2-50

H5-09

B5-24

H4-64

R2-36

E6-31

R2-36

55-07

R2-18

HI-30

R3-12

L6-10

B2-35

H5- 49

H5-53

R3-45

B3-63

S4-57

R2-50

$\times 5-52$

T4-07

R1-51

RI-30

R2-88

H4-65

T1-30 


\section{MHC-EP-0182-69 \\ Distribution - continued}

G. R. Priddy Jr.

R3-12

R. K. Quinn

$\mathrm{K} 1-73$

T. E. Ratney

R4-02

R. E. Raymond (3)

R2-54

R. W. Reed

I. E. Reep

RI-51

M. A. Rezvant

R2-08

D. Richerdson

H5-55

S. K. Rifaey

R2-31

56-12

W. E. Ross

55-07

J. A. Ryan

H5-55

P. Sathyanarayana

R2-12

C. C. Scalef

L7-06

F. A. Schmorde

B2-18

C. P. Schroeder

ᄂ7-06

K. V. Scott

H5-52

N. J. Scott-Proctor

R1-80

O. Serrano

T4-01

L. K. Severud

R2-50

M. H. Shannon

H4-61

J. E. Shapley

H4-68

T. N. Shaw

S4-55

R. A. Shea

L8-16

E. M. Sheen

ᄂ7-03

E. J. Shen

S4-58

P. K. Shen

HO-39

D. J. Sherwood

R2-78

A. T. Shook

E. R. Sictliano

S2-01

K. P. Slape

HO-39

C. M. Smith

SO-11

S. G. Spencer

H6-30

R1-30

S. M. Stahl

E. G. Stephan

H4-61

B. E. Stapley

A3-74

R. R. Stickney

R4-02

R4-02

J. N. Strode

R2-11

D. G. Sutherl and

L4-72

J. P. Sumnerhays

R2-88

M. J. Sutey

S. L. Swaney

T4-08

T4-08

L. M. Swanson

H5-49

H5-68

J. F. Thompson

RI-30

J. D. Thomson

H6-26

S. R. Tifft

J. A. Tilden (2)

L6-12

H. Toffer

HO-38

T. T. Tran

L7-04

J. W. Tritz

B1-32

D. P. Trott

B1-32

D. A. Turner

R2-78

L. A. Tusler

R2-11

C. J. Udell

L6-12

R. E. Van der Cook

L4-75 


\section{MHC-EP-01E:-69 \\ Distribution - continued}

R. J. Van Vieet

H4-62

D. T. VIadimiroff

G7-01

J. A. Voogd

G. R. Walker

R4-03

O. S. Wang

B2-16

D. L. Negener

R. K. Weity

G. T. Wells

R. Whitman

J. H. Wicks

R2-78

RI-62

RI-80

H6-26

H5-49

D. D. Wiggins

T4-07

L. S. Willians

RI-49

C. R. Wilson

S4-53

G. R. Wilson

H5-52

M. E. Witherspoon

R2-31

D. D. Wodrich

K. L. Noodard

R. D. Nojtasek

E6-61

R2-85

R4-02

W. R. Hrzesinski

H6-27

P. A. Young

R3-74

F. A. Zak

R2-14

B. D. Zimmerman

R3-01

LO-06

SDPC Docket File (2) H5-36

272-AW Shift Office 55-04

Central Files (2) L8-04

Tank Farms Info Center R1-20

Information Release

Administration (3) R1-08

Env Data Mgmt Center H4-22

RL/TWRS LIbrary R3-72 

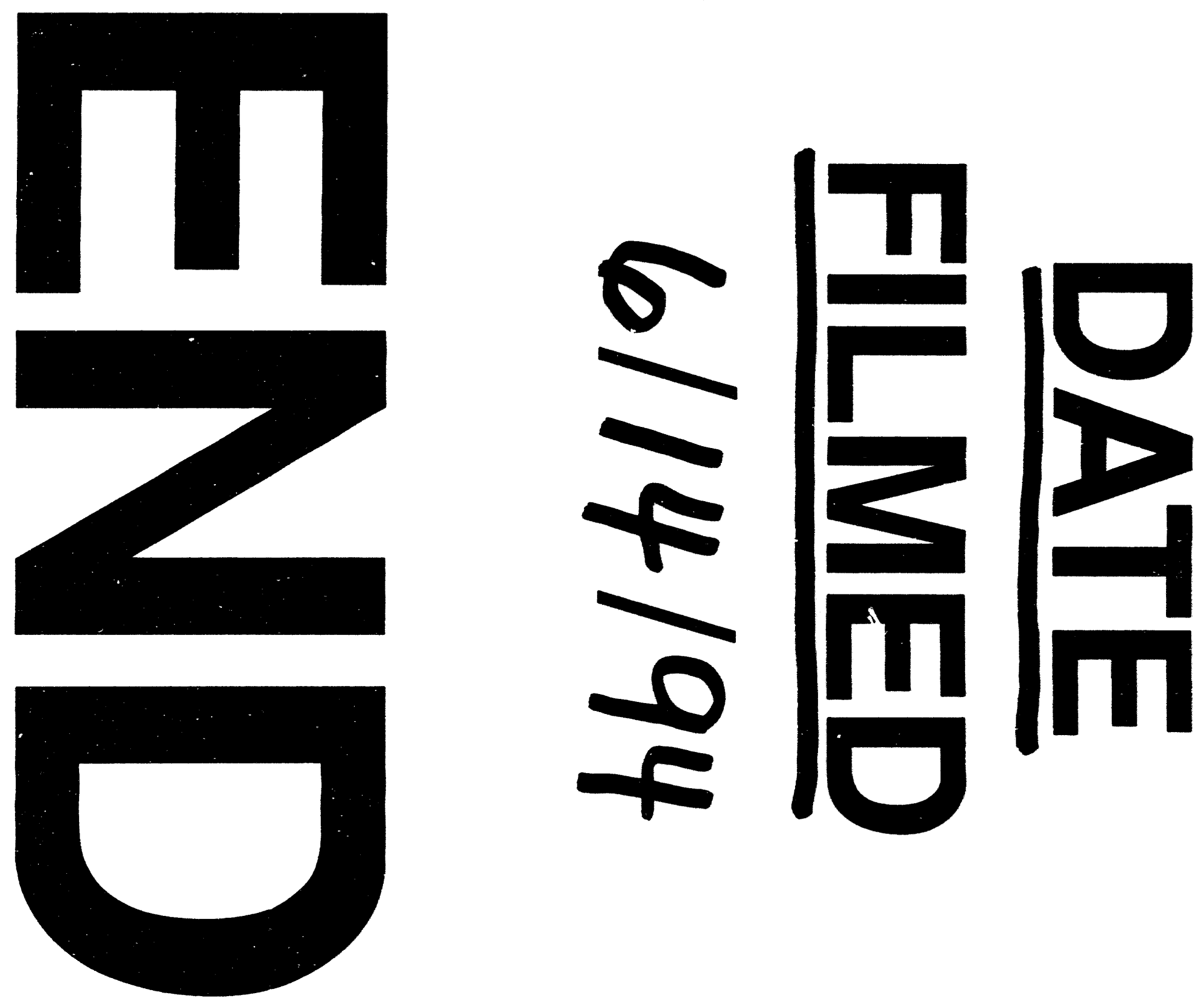